\title{
Model-Theoretic Accounts of Logical Consequence — Themes from Etchemendy —
}

\author{
Kasper Højbjerg Christensen
}

A thesis submitted to the Victoria University of Wellington in fulfilment of the requirements for the degree of Doctor of Philosophy

Victoria University of Wellington

2015 



\begin{abstract}
This thesis is a discussion and continuation of a project started by John Etchemendy with his criticism of Tarski's account of logical consequence. To this end the two central concepts of the thesis are those of an interpretational and representational model-theoretic account of logical consequence, respectively.

The first chapter introduces Etchemendy's criticism of Tarski's account of logical consequence, a criticism which turns essentially on an interpretation of Tarski according to which his proposed account gives rise to a purely interpretational model-theoretic account of logical consequence. Consequently there must be a representational aspect to our model-theoretic definition of logical consequence.

The second chapter introduces Etchemendy's notion of logical consequence: that of being truth preserving in virtue of the semantics of the involved terms. While this notion is representational, we argue that Etchemendy's notion of a categorematic treatment of terms reintroduces an interpretational aspect back into the model theory. The chapter investigates the resulting notion, compares it to other notions in the literature, and presents certain results that can be proved, under certain conditions, about this notion in relation to the notion of being truth preserving in virtue of the semantics of all terms.

Chapter three of the thesis is concerned with the question of how a standard model, seen as a domain and an interpretation function, manages to capture the different notions of model-theoretic consequence. As we explain, this question is most pressing
\end{abstract}


when we want our models to both represent and interpret, and we will present a theory which allows us to see the models as both representing non-actual possibilities as well as provide interpretations for the terms.

The fourth chapter applies the lessons of the preceeding chapters to argue that Kripke Semantics can be seen as capturing the notion of being truth preserving in all possibilities under all interpretations of the non-logical terminology in the case where our language is augmented with an operator, $\square$, to represent logical necessity. We will argue this point by contrasting it with, though not necessarily disagreeing with, claims made by various authors to the effect that Kripke Semantics is not the appropriate semantics when our language contains an operator for logical necessity. 
To JPNDP and BT. 



\section{ACKNOWLEDGEMENTS}

There are a great many people to whom I am grateful. Firstly, I would like to thank Ed Mares, Max Cresswell, and Adriane Rini for inviting me to Wellington to work with them on their project about necessity. From the very beginning Ed, Max, and Adriane have encouraged me to pursue the aspects which I found interesting within the broader topic. The fact that I have had the freedom to work on my topic of choice has greatly improved the finished product, and has, no doubt, made some of those very long nights seem less long. Thank you.

I owe a huge thank you to Ed and Max in their capacities as my supervisors here at Victoria. Both have made tremendous contributions to the ideas that eventually became this thesis, and I have benefitted greatly from our various conversations.

Ed has functioned as my primary supervisor, so in addition to the helpful conversations we have had, the valuable comments on drafts, suggestions, and advice, I also owe Ed a thank you for helping me on the administrative side of things. (Who knew there was so much paperwork involved in doing a PhD!). Thank you, Ed.

Max has functioned as my secondary supervisor, but only by title. Max has provided me with extensive written comments on a vast number of previous drafts of the present thesis. The patience with which he has done so has been admirable. I can only imagine the frustration it may have caused to see that I had once again misunderstood a crucial point or refused to take a crucial comment into account. The present thesis owes a great deal to Max's tireless efforts. Thank you, Max. 
I also owe a thank you to the members of my Thesis Committee: Cei Maslen, Christopher Menzel, and Patrick Girard. Besides taking the time to voluntarily read through the thesis, all three of them have provided useful comments and insightful suggestions for improvements, both at the defense and in writing. These suggestions have all ultimately helped make the thesis better. Thank you.

I would also like to thank the Philosophy Community at Victoria, both staff and students, for making me feel welcome here. I have benefitted greatly from your help along the way; whether it be comments on a seminar talk or the offer of a beer and a game of cards on a Friday afternoon.

When you spend three years in a certain place, you are bound to make some friends. I have made so many that by mentioning them here by name, I will surely forget some. I owe the biggest thank you to all of you. For keeping my spirits up in times of need and in general just for being awesome.

Lastly, I would like to thank my friends and family back in Denmark, as well as my family here. My mum and my sister deserve the biggest thank you of all. I know it is a cliché, but absolutely none of this would have ever happened without their love and support. 


\section{CONTENTS}

Introduction . . . . . . . . . . . . . . . . . . . . . . . 1

0.1 Logical Consequence . . . . . . . . . . . . . . . . 3

0.2 Models \& Model Theory . . . . . . . . . . . . . . . . . . 8

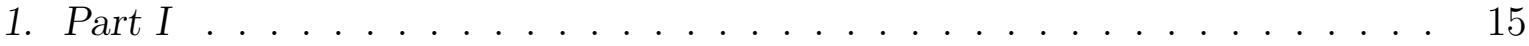

1.1 Tarski's Definition of Logical Consequence . . . . . . . . . . . . . 15

1.1.1 Adequacy of The Definition . . . . . . . . . . . . 25

1.1.2 Interpretational vs. Representational Semantics . . . . . . . . . 29

1.1.3 Interpretational Model-Theoretic Semantics . . . . . . . . . . . 36

1.1.4 Is Tarski's Account Interpretational? . . . . . . . . . . . . . . . 40

1.1.5 The Attractiveness of Tarski's Account . . . . . . . . . . . . . 44

1.2 Etchemendy's Criticism . . . . . . . . . . . . . . . . 49

1.2.1 What Etchemendy's Criticism is Not . . . . . . . . . . 57

1.2.2 Tarski's Fallacy . . . . . . . . . . . . . . . 59

1.2.3 Definitional vs. Epistemic Order . . . . . . . . . . . . . 63

1.2.4 The Reduction Principle . . . . . . . . . . . . . . . 69

1.2.5 Substantive Generalizations . . . . . . . . . . . . . . 73

1.2.6 The Contingency Problem . . . . . . . . . . . . . . 76

1.2.7 Alternative Formulation of the Source of Tarski's Fallacy . . . . 81

1.2.8 Dependent on Logical Constants . . . . . . . . . . . . . . . . . . 86 


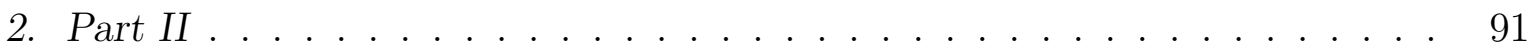

2.1 Etchemendy's Modeling Perspective . . . . . . . . . . . . . . . . . 92

2.1 .1 Not An Analysis . . . . . . . . . . . . . . . . . 95

2.1.2 Interpretational Character . . . . . . . . . . . . . 100

2.1.3 A Logically Possible Way The World Could Have Been . . . . . 105

2.1.4 What Is Part of The Semantics? . . . . . . . . . . . . . . 116

2.2 Logical Constants and The Isomorphism Property . . . . . . . . . . . 124

2.2.1 The Equivalence of Two Criteria . . . . . . . . . . . . . . 130

2.3 Meta-Theorems . . . . . . . . . . . . . . . . . . . 135

2.3.1 Squeezing Arguments . . . . . . . . . . . . . . . . . . . . . 142

2.3.2 Consequence in Natural Language . . . . . . . . . . . . . . . 145

2.3.3 Completeness by Default . . . . . . . . . . . . . . . . . . . . 149

3. Part III . . . . . . . . . . . . . . . . . . . . . . . . . . . . . 155

3.1 A Categorization . . . . . . . . . . . . . . . 157

3.1 .1 On Interpretations $\ldots \ldots \ldots \ldots$

3.1 .2 On Representations . . . . . . . . . . . . . . . . . 165

3.1.3 On Representations and Interpretations . . . . . . . . . . 167

3.1.4 A Possible Solution . . . . . . . . . . . . . . . . . . . . . 172

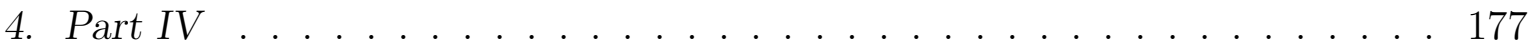

4.1 I-models \& E-models . . . . . . . . . . . . . . . . . . . . . . . . . 182

4.1 .1 Pure vs. Applied Semantics _. . . . . . . . . . . . 185

4.1.2 Etchemendy on Kripke Semantics . . . . . . . . . . . . . 189

4.1 .3 Logical Necessity . . . . . . . . . . . . . . . . . . . . . . . . 199

4.2 The Criticism of Kripke Semantics _ . . . . . . . . . . . . . . . 202

4.2 .1 Propositional S5 Semantics . . . . . . . . . . . . . . . . . 210 
4.2.2 There Are Valuations qua Logical Possibilities Missing . . . . . 216

4.2.3 There Is A Fixed Class of Logical Possibilities . . . . . . . . . . 228

4.3 Quantified Languages . . . . . . . . . . . . . . . . . . . 232

4.4 The Intended Frame . . . . . . . . . . . . . . . . . . . . . . . 246

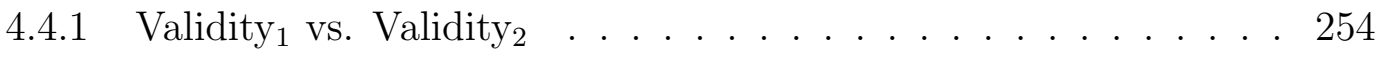

4.4.2 Logical Possibility is Non-Relative . . . . . . . . . . . . . 263

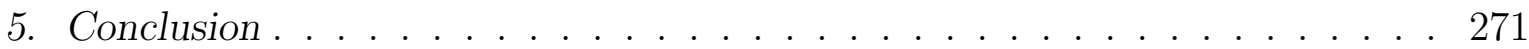

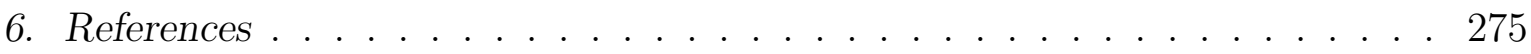





\section{INTRODUCTION}

The present thesis is a discussion of, and for some parts a continuation of, a project started by John Etchemendy with his criticism of Tarski's account of logical consequence (Tarski [1956a]). The whole of Etchemendy's project is essentially linked to his crucial distinction between an interpretational model-theoretic account of consequence and a representational model-theoretic account of logical consequence.

In order to use Etchemendy's distinction between representational and interpretational model-theoretic semantics, I must first explain what the distinction amounts to, and in order to do so my starting point will be Etchemendy's criticism of Tarski's account, a criticism which has been the focal point of much of Etchemendy's work (Etchemendy [1982, 1983, 1988a, 1988b, 1990, 2008]). The first chapter will introduce Tarski's definition of consequence, and explain Etchemendy's criticism of this notion. Through presenting both Tarski's account and Etchemendy's criticism it should become clear what an interpretational semantics amounts to, and why a purely interpretational model-theoretic semantics does not suffice in capturing the concept of logical consequence.

Chapter 2 will present Etchemendy's positive views of model theory, and compare it to a few other theories in the literature, most prominently, that of Shapiro [1998, 2001, 2002, 2005]. This is done primarily to point out that Etchemendy's criticism is not a criticism of model-theoretic semantics per se, but of a certain view of what the underlying models represent. By presenting Etchemendy's positive view, a view which 
takes the model theory to be essentially representational, we aim to make this point clear, but the presentation is also needed to introduce Etchemendy's notion of the categorematic treatment of some terms, a treatment which introduces an interpretational aspect back into the model theory.

Assuming a certain notion of what a model-theoretic characterization of consequence aims to do, we are able to defend the use of Kripke Semantics in giving a semantics for a $\square$-operator understood as logical necessity. This defense will be the main focus of chapter 4 , where we will also point out that some other peculiar phenomena regarding Kripke Semantics, phenomena which might otherwise have been considered unmotivated, or even false, are welcomed consequences of applying Etchemendy's modeling perspective to the resulting model-theoretic consequence relation.

While Etchemendy does stress that models represent various ways the world could have been, he does not provide any positive account as to how the models manages to do so. This question becomes pressing when we want the models to both represent and provide interpretations for the non-logical constants. Chapter 3 discusses how we can see models as providing interpretations and how we can see them as representing other possible ways the world could have been. We also present a theory of how we can see the models as both representing and interpreting at the same time, something which seems to be crucial to Etchemendy's modeling perspective. 


\subsection{Logical Consequence}

Logical consequence is the key notion in the philosophy of logic. ${ }^{1}$ What does it mean to say that something follows logically from something else? To answer this question we must first say something about what the entities that can follow logically from others are. The present thesis will have nothing directly to say about this matter. This is the problem of the relata of logical consequence. ${ }^{2}$ Are the relata of logical consequence propositions, or perhaps declarative sentences? If so, are the propositions structured? Are the declarative sentences types or tokens? These, and countless other questions, are relevant when it comes to deciding what it is that can properly be said to follow from something else: what is the nature of the things that can stand in the logical consequence relation?

If one assumes, quite naturally, that logical consequence is about truth-preservation, the question of what the logical consequence relates becomes a question of what can properly be said to be true or false. Here, of course, we encounter the same kind of candidates as before: propositions, sentence types, sentence tokens, and so on.

In this thesis we will try to remain as neutral as possible on the question of the relata of the logical consequence relation. We will assume that declarative sentences of

\footnotetext{
${ }^{1}$ By which we do not mean to commit ourselves to the view that it is the only interesting notion in the philosophy of logic. There are obviously countless other important philosophical questions concerning logic, some of which are related to the notion of logical consequence, and others which are, at least on the face of it, not. "What is a logical constant?", "What is truth?", "Is set theory logic?", "Is classical logic the correct logic?", "Should everything follow from a contradiction?" are just a few examples of questions that are obviously important, but that will only play a role in the present thesis as they relate to the notion of logical consequence which is our primary focus.

Concerning the latter two questions, the reader will quickly discover that we assume a classical notion of model-theoretic consequence in the present thesis. This is not meant to imply a positive answer to the question "Is classical logic the correct logic?" (and thereby also a positive answer to the question "Should everything follow from a contradiction?"), but serves merely to provide a common framework in which to address and discuss the issues at hand.

${ }^{2}$ See Russell [2008] for a discussion of the problem and the various candidates. Russell argues that none of the obvious candidates - sentences, propositions, pairs of sentences and truth values, etc.can make sense of our logical practice, and argues that giving different answers to the problem will generate different logics. Russell thus argues for a kind of logical pluralism.
} 
natural language (in our case English) can, at least in a derivative sense, possess the property of logical truth and stand in the relation of logical consequence. Whether or not we think of these as tokens or types will not matter much for our purpose, though one could argue that types are not in fact declarative in that a given context is needed for them to declare anything, that is, to express a fact. If it turns out that declarative sentences, in some underspecified sense, are the primary bearers of truth, there is no problem. But the idea is that even if it turns out that propositions are the primary bearers of truth, we can nevertheless make sense of declarative sentences standing in the logical consequence relation. This happens if and only if the propositions they express stand in this relation. We can note, though, that if propositions really are the primary truth bearers and the relata of logical consequence, then sentence types will not derivatively stand in the consequence relation or express logical truths, since types do not by themselves express propositions. If this is the case we should therefore think of a declarative sentence as a sentence type and a context, or whatever else the linguistic phenomenon we take to express propositions is. What matters, is that logical consequence can obtain between bits of language. We will refer to these bits as 'statements', though at other points we will simply call them 'sentences'.

By this we do not want to limit ourselves to the view that only statements, in this sense, can stand in the logical consequence relation to one another. The minimal assumption of this thesis is that statements can-perhaps in this derivative sense- stand in this relation, not that they are the only thing that can.

Logical consequence, in the sense in which we will be interested in this phenomenon, is a relation between a class of statements, $K$, of natural language, called the premises, and a single statement, $X$, which we call the conclusion. ${ }^{3}$ We can now ask what it means

\footnotetext{
${ }^{3}$ The decision to work only with single statement conclusions is not meant to carry any philosophical commitment. We could just as well have worked with a set of conclusions; containing no statements
} 
for $X$ to follow logically from $K$. One feature which is often mentioned when logically valid arguments are at stake, is the fact that it is impossible for a valid argument to have true premises and a false conclusion. Or, in other words, logically valid arguments preserve truth necessarily.

There are other features, besides necessity, which are often claimed as key features of a valid argument. For example, it is often claimed that if logical consequence obtains between the class of statements $K$ and the statement $X$, then one should be able to know this a priori. The idea is that whether logical consequence obtains should not be influenced by empirical facts, and consequently, if it obtains it obtains independently of such facts, and knowledge of empirical facts are therefore irrelevant to knowing whether it obtains. Solely by reflecting on the statements in $K$ one can come to know that if they are true, then so is $X$. There are different ways of making this idea precise, and there are those who deny that logical consequence is a relation that can always be known to obtain a priori. Some claim that it cannot always be known that a given statement follows logically from a given class, and the claim is often qualified to: logical consequence can be known to obtain a priori, if it can be known at all (see for example Hanson [1997: 378]).

The idea that knowledge of logical consequence is a priori often goes hand in hand with the slogan that logic is formal. If we accept that whether or not the relation obtains is independent of the empirical facts, one naturally is led to the idea that the exact objects being referred to in the involved statements are inessential. For this reason we can replace a term designating a certain object by a term designating another object, and still have a valid argument, as long as this replacement is uniform. This

(i.e., being the empty set) or containing multiple statements, but for matters of simplicity we choose to treat only singleton sets as conclusions, or, what that amounts to, we always have a single statement as the conclusion. 
gives us the idea of a logical form.

The logical form of an argument is given by presence of the so-called logical terms of the language in question, and the validity of a given argument is a function of the logical form which the argument has. This idea has been prominent in the literature (Tarski [1956a], Sher [1991, 1996, 2008]), but there are those who reject that logical form is capable of playing this kind of prominent role in a theory of consequence (Etchemendy [1983], Read [1994]).

Besides these there are other features of a valid argument which are mentioned in the literature. It is, for example, often claimed that logic is normative. If you accept the premises of a valid argument and reject its conclusion you are somehow doing something wrong (see for example Beall \& Restall [2006: 16]).

The most prominent feature of a logically valid argument is that such an argument guarantees that its conclusion is true if its premises are. This is what makes logically valid arguments so useful. We can extend our knowledge by constructing valid arguments with true premises and consequently be guaranteed that the conclusions must be true as well. The whole enterprise of logic as a science of reasoning and arguments is founded on the simple observation that some arguments are good in this sense: that they provide prima facie evidence for their conclusions, they guarantee that their conclusions are true if their premises are.

This guarantee which a logically valid argument possesses is often spelled out as a modal connection between the premises and conclusion. The idea is that it is impossible for the premises of a valid argument to be true while the conclusion is false. Or, equivalently, that it is necessary that if the premises of a valid argument are true, then so is the conclusion. If it is impossible for the premises of a valid argument to be true while the conclusion is false, then one is certainly guaranteed that if the premises are 
true so is the conclusion, since there are no possible circumstances in which this is not the case.

There are various ways of giving content to the modality in question. It is sometimes claimed that logical necessity, the necessity with which the conclusion of a valid argument must be true if the premises are, is the strongest kind of necessity (McFetridge [1990]). ${ }^{4}$ What this means is that if something is logically necessary, then there is no other sense of necessity in which it is not necessary, such as nomic or metaphysical. Once this is established it is standard to further claim that all other notions of necessity are relative to logical necessity, in that they follow from the logical necessities in combination with some body of truths, $\Phi$ (Hale [1996: 93ff]). $\Phi$ could for example be the laws of physics, and we would thereby define the notion of nomic necessity relative to logical necessity.

We shall later argue that whatever the modal element in a logically valid argument is, it has to extend beyond the actual world in order to make sense of our logical practice. Logic is standardly taken to apply not only in actual circumstances but also in merely hypothetical circumstances, circumstances that do not actually obtain. If valid arguments are to carry a guarantee that in these non-actual circumstances if the premises are true, so is the conclusion, the modality in question must range over non-actual circumstances. The claim that logical necessity ranges over non-actual circumstances is therefore not as strong as the claim that logical necessity is the strongest notion of necessity, but it will follow from this latter claim if accepted.

\footnotetext{
${ }^{4}$ In fact, McFetridge [1990] argues for two claims: the claim that if there is a notion of necessity involved in the claim that an argument is valid, then that is the strongest notion of necessity, and the claim that we ought to believe that there is such a notion of necessity involved in a valid argument.
} 


\subsection{Models \& Model Theory}

In the present thesis we will concern ourselves with model-theoretic characterizations of logical consequence. By such a decision we do not want to convey that there is nothing interesting about proof-theoretic characterizations of logical consequence. One of the main points of the thesis will be that model theory aims to capture an interesting notion in natural language that is worth studying in its own right. Nothing is thereby said about proof-theoretic characterizations being able to capture or analyze other (or perhaps the same) interesting notions that are also worthy of our attention.

A model theory is a collection of objects called models. Sentences of a formal language are said to be true or false in models, and we define a model-theoretically valid argument as one that preserves truth in all models. Let $\Gamma$ be a class of formulas of a formal language and $\alpha$ just one such formula. Writing $M \Vdash \gamma$ for the formula $\gamma$ being true in $M$, our model-theoretic definition of logical consequence, which will be the prime focus of the present study, is the following:

- $\alpha$ is a model-theoretic consequence of $\Gamma$, written $\Gamma \models \alpha$, if and only if for all models $M$; if $M \Vdash \gamma$ for all $\gamma \in \Gamma$, then $M \Vdash \alpha$.

A model-theoretically valid argument is thus one such that it preserves truth in all models. Or, what is equivalent, there is no model in which the members of $\Gamma$ are true but $\alpha$ is false.

Model theory has been studied extensively, since Tarski arguably laid most of the groundwork for model theory to become a discipline in its own right through his work

in the 30's (Tarski [1956a, 1956b]). Hodges [1997] contains an overview of many of the mathematical results that have since been proved.

In the present thesis we will be concerned with how the model-theoretic definition 
of logical consequence relates to arguments in natural language. This decision is not meant to imply that there are no other interesting uses of model theory and modeltheoretic definitions of logical consequence, but the focus of this thesis is on the notion of logical consequence as it is found in natural language. The decision to so focus is based on nothing more than the simple observation that arguments, as we normally understand them in philosophy, are, after all, carried out in a natural language, and we would therefore like to know what sets a good natural language argument, in the sense that the truth of its premises guarantees the truth of its conclusion, apart from an argument for which this is not the case.

The expressions of natural language are the kind of things that have meanings, and combined in the right kind of way they form statements that can be true or false. Formulae of a formal language, however, are just mathematical symbols, and though they may be generated in accordance with a definite syntax, they do not really mean anything, much less are they able to express anything which can properly be said to be true or false.

Here, of course, is where the notion a model-theoretic semantics comes in, a notion which has grown out of Tarski's work. A model is a collection of objects of some sort with a structure imposed on them which relates the objects to the expressions of the formal language. That is, a model, $M$, is a pair $M=\langle D, I\rangle$, where $D$ is a non-empty set of object and $I$ assigns appropriate members drawn from $D$ to the terms of the formal language. To link the models and the formal language to that which is expressed by natural language we simply let the objects in $D$ be the objects our natural language talks about and let the denotations assigned to our non-logical terms by $I$ match the meanings of our natural language terms.

Suppose our formal language contains a predicate german, which is meant to reflect the natural language expression 'is German'. What makes the latter mean what it 
means is presumably that it truly applies to objects that are German. This suggests the following straightforward treatment of german as a formal term: in order to make it behave like 'is German' behaves in natural language, we simply assign it the set of German objects that are members of $D$. That is, $I$ (german $)=D \cap\{x \mid x$ is German $\}$. In a similar fashion, supposing our formal language contains the name marx, which is meant to be the formal counterpart of the name 'Karl Marx', we simply suppose that Karl Marx is in $D$ and that $I(\operatorname{mar} x)=$ Karl Marx. This gives us a straightforward link between the truth of the natural language statement 'Karl Marx is German' and the formula german(marx), which, by the truth clauses for our formal language is to be true in $M$ if and only if $I(\operatorname{mar} x) \in I($ german $)$. What makes german and marx take on the meaning of the corresponding natural language expressions in $M$ is that marx is assigned the actual object that is Karl Marx whereas german is assigned the set of German people.

In this way we can add even more formal counterparts of natural language expressions. We can add the formal counterparts of terms like 'and', 'or', 'not', 'all', and the like, and link their contribution to the truth or falsity of natural language statements in the appropriate manner to their contribution to the truth or falsity in any given model. For example, the formal counterpart of 'and', which we write $\wedge$, gets the following truth clause in a model $M$ for formulas $\alpha$ and $\beta . M \Vdash \alpha \wedge \beta$ if and only if $M \Vdash \alpha$ and $M \Vdash \beta$. This straightforwardly reflects that a natural language statement ' $\int_{1}$ and $\int_{2}$ ' is true when both natural language statements $\int_{1}$ and $\int_{2}$ are true. ${ }^{5}$

Terms such as 'and', 'or', 'not', 'all', and the like are often designated as logical terms. What this means is that their formal counterpart is treated indifferently in all

\footnotetext{
${ }^{5}$ In the thesis we give the standard classical semantics for the logical constants. This is not meant to convey that it is a settled matter that this is the correct choice. If anything, the matter here is highly controversial. The choice to give a classical model-theoretic semantics serves merely to deliver a common framework in which to address and discuss the issues that are the main focus of the present thesis.
} 
models. The truth of $\alpha \wedge \beta$ in $M$, for example, is decided with an implicit quantifier over models, and is therefore given without reference to any particular model. This, as we saw, is not the case with german(marx), because it matters what the interpretation function, $I$, assigns to these formal terms.

We can also add relations, like loves, to our formal language and assign as their extensions sets of tuples drawn from $D$. Assuming that this is done so that $I$ (loves $)=$ $D^{2} \cap\{\langle x, y\rangle \mid x$ loves $y\}$, we get a perfectly good explanation, in model-theoretic terms, of why (kasper)loves(marx) is not true. I do not, as a matter of fact, love Marx, so $\langle$ Kasper, Marx $\rangle \notin I($ loves $)$.

In the above examples we have had a fully interpreted formal language in mind. It was already decided from the outset that german was to be the formal counterpart of 'is German' in natural language. But it is clear how we can also speak about interpretations of formal language terms whose meaning is not determined prior to setting up the model theory. Consider the formal predicate $F$. In a model, $M$, where $I(F)=D \cap\{x \mid x$ is Danish $\}$ we can say that $F$ is being interpreted as the formal counterpart of 'is Danish'. $F$ means 'is Danish' in $M,{ }^{6}$ or the model $M$ interprets $F$ as the set of Danish people. Similarly, we can speak about interpretations of a term like german, even if it is not interpreted to refer to the set of German people. If $I($ german $)=D \cap\{x \mid x$ is Danish $\}$, then german is interpreted as 'is Danish' in the given model. For this reason we might want to call it a reinterpretation, but nothing crucial hangs on whether we do.

Tarski's account of consequence, given in contemporary model-theoretic terms, is to say that $\alpha$ is a logical consequence of $\Gamma$ if and only if, when a model interprets all of $\Gamma$ 's formulae to be actually true, it interprets $\alpha$ to be actually true.

\footnotetext{
${ }^{6}$ We should perhaps be careful about such phrases as ' $F$ means ...'. It is generally agreed that meaning is an intensional notion, and that an extension is consequently insufficient in order to determine meaning. Hence our emphasis on the fact that $F$ means 'is Danish' in $M$.
} 
As we shall see Etchemendy claim, this is not enough to characterize the notion of an argument that preserves truth with necessity, and consequently, an argument that guarantees that the conclusion is true whenever the premises are. For this reason Etchemendy criticizes Tarski's account of consequence. Tarski's account takes into considerations only actual interpretations of the non-logical constants, and consequently we are not guaranteed that the valid arguments have the requisite modal profile, that is, that they carry any weight in how things have to be if they are a certain other way. This point will be explained in great detail in the course of the thesis.

To secure this, Etchemendy urges, we must view the models not as reinterpretations of the non-logical constants, but as representing other possible ways the world could have been. If our models somehow represent all and only possible ways the world could have been, then we can be sure that valid arguments are truth preserving in all possibilities and so possess the requisite modal profile.

An easy way to make the requisite adjustment would be to have the entities of our models simply be the entities from the possible worlds in question. A model could then represent Karl Marx as being Russian by having the Marx of the possible world in which he is Russian be the value of $I(\operatorname{mar} x)$ and the set of Russian people in that possibility be the value of $I$ (russian). russian(marx) would consequently be true in that model. But, of course, this seems to commit us to the existence of these merely possible objects that can play the roles of the entities from which we build our models.

If we reject that these are available, however, it seems that we lose the most straightforward way of explaining the relationship between our natural language statements and our formulae. If there are, say, no unicorns, then how can we make the formal language predicate unicorn mean in $M$ what 'is a unicorn' means in natural language? We can no longer claim that it does so by being assigned the members of $D$ that are unicorns, since there are, per assumption, no such members. The reader might feel that 
this is as it should be. After all, there are no unicorns, so the expression 'is a unicorn' does in fact pick out the empty set. From the perspective of seeing models as actual interpretations of the language, this would be sufficient, but we should remember that the models are now meant to represent ways that the world could have been. And if there could have been unicorns, we cannot provide the above straightforward link between natural language and truth in a model.

In this case we would have to say that an entity can represent, or be a stand-in for, in some yet underspecified way, a unicorn. One idea that springs to mind is that a given entity, which is not a unicorn, could represent a unicorn simply by being in the set I(unicorn). This, of course, requires a fully interpreted formal language, since it is relative to the meaning of unicorn, that members of $D$ represent unicorns. Even in the case where we do have a fully interpreted formal language in mind, we seem to run into trouble if our models should provide reinterpretations for some terms. How do we interpret the predicate $F$, which per assumption means something different than unicorn, to mean 'is a unicorn' if there are no unicorns in $D$ and we want to represent there being a unicorn? In this case we cannot claim that the members of $I(F)$ represent unicorns, for the meaning of 'is a unicorn' was never attached in the requisite manner to $F$. It seems, then, that there must be something extra to the model, i.e., something not given by $D$ or $I$, which makes it so that a member of $D$ can represent a unicorn.

In chapter 3 we will investigate these issues in some detail, and present a way in which we can make sense of a model as both representing a non-actual circumstance and providing an interpretation of the non-logical terms. 



\section{PART I}

\subsection{Tarski's Definition of Logical Consequence}

Tarski starts his celebrated landmark essay (Tarski [1956a]) by noting that the concept of logical consequence is not one that it is open for any theorist to define into existence as he pleases.

The concept of logical consequence is one of those whose introduction into the field of strict formal investigation was not a matter of arbitrary decision on the part of this or that investigator; in defining this concept, efforts were made to adhere to the common usage of the language of everyday life. (Tarski [1956a: 409])

Tarski is pointing out that the concept of logical consequence has a prior everyday usage which any theorist who wishes to develop a strict formal definition must seek to respect. ${ }^{7}$ Tarski also points out that we cannot expect any one definition to be able to respect all and every usage of the concept as it is found in everyday language.

With respect to the clarity of its content the common concept of consequence is in no way superior to other concepts of everyday language. Its extension is not sharply bounded and its usage fluctuates. Any attempt to

\footnotetext{
${ }^{7}$ Etchemendy contrasts the situation with that of the definition of a group or a real closed field as they are introduced into mathematics (Etchemendy [1990: 9]). The point here is arguably that these terms had no prior usage to respect and that the mathematician was free to define them as he pleased. The situation, for the concept of logical consequence, is not like that.
} 
bring into harmony all possible vague, sometimes contradictory, tendencies which are connected with the use of this concept, is certainly doomed to failure. We must reconcile ourselves from the start to the fact that every precise definition of this concept will show arbitrary features to a greater or less degree. [1956a: 409]

Tarski is suggesting here that any strict formal definition of logical consequence must in a certain sense be revisionary: it cannot possibly respect all and every everyday language usage, since these usages are sometimes contradictory, and often the judgments made are fluctuating. One argument declared valid may be declared invalid at a later time or by another person.

With these preliminary comments out of the way Tarski jumps headlong into a discussion of previous attempts to define, in a rigorous and formal manner, a concept of following logically. Tarski notes that until recently a great many logicians had thought that they had succeeded "by means of a relatively meagre stock of concepts, in grasping almost exactly the content of the common concept of consequence, or rather in defining a new concept which coincided in extent with the common one" [1956a: 409]. ${ }^{8}$ Tarski is here referring to proof-theoretic characterizations of consequence, where a sentence is a logical consequence of other sentences if and only if it can be obtained by applications of simple rules of inference to the sentences themselves and/or the axioms of the system. As evidence for the correctness of such a definition, Tarski says, "the logicians were able to bring forward a weighty argument: the fact that they had actually succeeded in reproducing in the shape of formalized proofs all the exact reasonings which had

\footnotetext{
${ }^{8}$ Since Tarski never names any of the "great many logicians" we are left with speculating on exactly who he had in mind. It is perhaps natural to assume that Tarski was thinking of logicians such as David Hilbert and the school surrounding him. However, for our purposes it is not essential who Tarski had in mind when making this comment. What is essential is that Tarski is rejecting proof-theoretic characterizations of consequence.
} 
ever been carried out in mathematics" [1956a: 410].

To counter the intuition that structural (i.e., syntactic) rules of inference are enough to capture the given concept of consequence, Tarski presents an example of an $\omega$ incomplete theory. In Tarski's example all sentences of the form $A_{n}$ : ' $n$ possesses the property $P^{\prime}$, for all natural numbers $n$, are provable, yet the universal sentence $A$ : 'Every natural number possesses the property $P$ ' is not provable ([1956a: 410ff]). Tarski claims that the universal sentence $A$ is a logical consequence of the totality of sentences $A_{0}, A_{1}, \ldots, A_{n}, \ldots$, but $A$ cannot be deduced from $A_{0}, A_{1}, \ldots, A_{n}, \ldots$ using the rules of inference and axioms of the given theory. Tarski considers the possibility of amending the rules of inference to allow us to infer $A$ from these given sentences, but quickly gives up on such a line of reasoning in the light of Gödel's incompleteness theorem.

In every deductive theory (apart from certain theories of a particularly elementary nature), however much we supplement the ordinary rules of inference by new purely structural rules, it is possible to construct sentences which follow, in the usual sense, from the theorems of this theory, but which nevertheless cannot be proved in this theory on the basis of the accepted rules of inference. [1956a: 412-413]

As Tarski points out, in the light of Gödel's incompleteness result, there will always be a sentence of a given theory (again, provided the theory is not of a particularly elementary nature) which follows in the usual sense from the theorems but which can nevertheless not be proved on the basis of those no matter how much we supplement the rules of inference. The assumption that proof-theoretic methods can capture all genuine logical consequences is therefore mistaken. ${ }^{9}$

\footnotetext{
${ }^{9}$ Before completely leaving Tarski's discussion of proof-theoretic methods, we might want to note that there is a certain tension in Tarski's example of an $\omega$-incomplete theory, Gödel's results, and
} 
Tarski starts the presentation of his own suggested definition with some intuitive considerations.

Consider any class $K$ of sentences and a sentence $X$ which follows from the sentences of this class. From an intuitive standpoint it can never happen that both the class $K$ consists only of true sentences and the sentence $X$ is false. Moreover, since we are concerned here with the concept of logical, i.e. formal, consequence, and thus with a relation which is to be uniquely determined by the form of the sentences between which it holds, this relation cannot be influenced in any way by empirical knowledge, and in particular by knowledge of the objects to which the sentence $X$ or the sentences of the class $K$ refer. The consequence relation cannot be affected by replacing the designations of the objects referred to in these sentences by the designations of any other objects. [1956a: 414-415]

According to Tarski these are essential features of the proper concept of logical consequence. Tarski takes them to be captured in condition $(\mathrm{F})$ :

Tarski's own definition of consequence. The issue is this: the inference from $A_{0}, A_{1}, \ldots, A_{n}, \ldots$ to $A$ looks to be just the inference from $P(0), P(1), \ldots, P(n), \ldots$ to $\forall n P(n)$. However, this is hard to reconcile with Tarski's knowledge of Gödel's completeness results for first-order languages, and the fact that this inference is not valid on Tarski's own definition of consequence unless the names for the natural numbers are treated as logical constants. Anything said against the proof theoretic conception at this level transfers to Tarski's own account via Gödel's completeness theorem. Etchemendy argues that this shows that Tarski did see the division between logical and non-logical terms as highly arbitrary and would allow distinct treatments for individual constants depending on what objects they name, that is, those naming natural numbers are allowed to be logical constants, but not so if they name, say, ordinary people, since this would threaten condition (F) which we will explicate below (See Etchemendy [1988b: 70ff]). Gómez-Torrente, on the other hand, argues that while Tarski did not see the names of the natural numbers as logical constants in the above inference, he did treat the natural numbers as defined in terms of the constants of a higher order set-theoretic language. (The most common example is defining 0 to be the empty set, 1 to be the set containing 0,2 to be the set containing 0 and 1 , and so on). This would make the inference higher order, meaning that Gödel's completeness result will not apply, as well as making it valid on Tarski's own account (See Gómez-Torrente [1996: 127ff]). I do not wish to take sides on this debate; see Edwards [2003] for a discussion of the issues. 
(F) If, in the sentences of the class $K$ and in the sentence $X$, the constants apart from purely logical constants — are replaced by any other constants (like signs being everywhere replaced by like signs), and if we denote the class of sentences thus obtained from $K$ by ' $K$ ', and the sentence obtained from $X$ by ' $X^{\prime}$ ', then the sentence $X^{\prime}$ must be true provided only that all sentences of the class $K^{\prime}$ are true. ${ }^{10}$ [1956a: 415]

Tarski explicitly states that condition (F) is a necessary condition for $X$ to follow logically from the class of sentences $K$, and goes on to ask whether it could be considered sufficient as well, in which case we would have succeeded in giving an adequate formal definition of the concept of logical consequence. ${ }^{11}$ However, as Tarski points out, treating condition $(\mathrm{F})$ as a sufficient condition for the sentence $X$ to follow from the class of sentences $K$ yields the wrong result in some cases [1956a: 415ff]. Though Tarski does not give an example of such a language, it is a relatively easy exercise to construct one. Consider a language containing as non-logical constants only the name 'Abraham Lincoln' and the two predicates 'was an American president' and 'had a beard' ${ }^{12}$ Now consider the argument $\langle\{$ 'Abraham Lincoln had a beard' $\}$,'Abraham Lincoln was an American president' $\rangle{ }^{13}$ The possible arguments that can be obtained from this by replacing non-logical terms for non-logical terms will have either 'Abraham Lincoln had a beard' or 'Abraham Lincoln was an American president' as the single premise and likewise for its conclusion. Since all the involved sentences are true all four arguments

\footnotetext{
${ }^{10}$ Here 'like signs being everywhere replaced by like signs' means that this replacement must be uniform, but also that it must respect the semantic category of the term that we replace. That is, predicates must be replaced by predicates, names by names, etc.

${ }^{11}$ In this case, Tarski notes, "[t]he only difficulty would be connected with the term 'true' which occurs in the condition (F). But this can be exactly and adequately defined in semantics" [1956a: 415]. Tarski is here referring to his own definition of truth and satisfaction as given in Tarski [1956b], as this is referenced in an accompanying footnote.

12 This is a variation on an example from Etchemendy [1990: 30ff].

13 Throughout this thesis I use the structure $\langle K, X\rangle$ to denote the argument with the class $K$ as premises and the sentence $X$ as conclusion.
} 
are truth-preserving, and the argument will satisfy condition (F). But it should be clear that the original argument is not valid. It does not follow from Abraham Lincoln having a beard that he was also an American president. So, satisfying condition (F) cannot be taken to be a sufficient condition for logical consequence to obtain.

Now, as Tarski notes, these kinds of counter-examples to treating condition (F) as sufficient, would not have been constructible had the language contained an expression designating each and every object. Since Karl Marx had a beard but was never an American President, had our language contained a name for Marx, we could have constructed a counterexample to the above inference by substituting the name for Marx for 'Abraham Lincoln'. The intention behind condition $(\mathrm{F})$ is on the right track in producing a sufficient condition, but it still makes the extension of logical consequence depend on the language in question. ${ }^{14}$ We would therefore like a way to express the intension behind condition $(\mathrm{F})$, though without having to rely on the expressive power of the language under consideration. A way to do just this, Tarski claims, is provided by semantics, especially his own notion of satisfaction of a sentential function by a sequence of objects, as developed in Tarski [1956b]. ${ }^{15}$

I will not attempt a full rehearsal of Tarski's notion of satisfaction. ${ }^{16}$ For our purposes, the intuitive workings of the notion of satisfaction will be sufficient. Consider a sentence of an interpreted language, say, 'Aristotle was the teacher of Alexan-

\footnotetext{
${ }^{14}$ Because Tarski's definition is ultimately a way of making the rationale behind condition (F) a sufficient condition for logical consequence to obtain, it is not uncommon to have Tarski's theory presented as one in which we expand the language such that it contains a term designating every object and then treat condition $(\mathrm{F})$ as necessary and sufficient for when $X$ follows from $K$. Technically, of course, this would be incorrect as the formal languages we consider are not expanded in any way on Tarski's approach: the underlying formal language contains a certain stock of non-logical constants and that stock remains stable even when Tarski's definition is applied. However, when applying the lessons from the next section we will see that, on Tarski's approach, we let the current language refer to entities that there are not necessarily any designators for in the language. The upshot is therefore the same, and we can justify conflating the two ways of presenting Tarski's definition.

${ }^{15}$ See in particular $\S 4$.

${ }^{16}$ Instead, I refer the reader to Tarski's original (Tarski [1956b: $\left.\S 4\right]$ ) for details.
} 
der'. The sentential function obtainable from this sentence is the sentential function we obtain by replacing the non-logical terms by variables of appropriate kind. If we suppose both 'Aristotle' and 'Alexander' are non-logical constants, we obtain the sentential function ' $x_{1}$ was the teacher of $x_{2}$ '. Now, consider the sequence of objects $\langle$ Aristotle, Alexander $\rangle$. This sequence, we say, satisfy the sentential function ' $x_{1}$ was the teacher of $x_{2}$ ' because the first object of the sequence, in this case Aristotle, was indeed the teacher of the second object of the sequence, Alexander.

Now, this picture is so far a bit too simplistic, but we may note that we have made no use of the fact that both Aristotle and Alexander have designators in our language (that is, English). What matters is that Aristotle is the teacher of Alexander, something which is the case even if we imagine our language not containing a means of referring to Aristotle and Alexander. This move, then, is essentially what allows Tarski to capture the intention behind condition $(\mathrm{F})$ without being dependent on the expressive resources of the language in question.

There are a couple of ways in which we need to extend this notion of satisfaction by a sequence of objects in order to make Tarski's definition of logical consequence clear. First we may note that for matters of satisfaction of the sentential function ' $x_{1}$ was the teacher of $x_{2}$ ' the order of the objects matters. The sequence $\langle$ Alexander, Aristotle $\rangle$ does not satisfy the sentential function since Aristotle was never taught by Alexander. We have implicitly been assuming that the variable $x_{1}$ matches up with the first object of a sequence, whereas $x_{2}$ matches up with the second. We can also see that whether or not there is a third, fourth, or $n$th element of the sequence does not matter for whether or not a sequence will satisfy ' $x_{1}$ was the teacher of $x_{2}$ '. The language may contain countably many expressions belonging to the semantic category of names, and it follows that the obtainable sentential functions may contain an arbitrary number of name-variables. Consequently, since we want a verdict on whether each sequence, as 
we are defining them, satisfies all possible sentential functions that can be obtained from sentences of the language, we work with infinite sequences. ${ }^{17}$ Any sequence, $\langle$ Aristotle, Alexander, ... $\rangle$, thus satisfies the above sentential function regardless of how the sequence is continued beyond the first two objects.

Secondly, we need to extend this notion so that we can handle more than just expressions belonging to the category of names. To do this we can introduce a different kind of variable for expressions belonging to different categories. So, for instance, we may use the variables $g_{1}, g_{2}$, and so on, to replace expressions that are in the category of 1-place predicates - i.e., '... is happy', '... is a prime number', and the like-, and $f_{1}, f_{2}$, and so on, for expressions in the category of relational predicates - i.e., '... is the brother of ...', '... killed ...', and the like. Consider, for example, the sentential functions ' $x_{1} f_{1} x_{3}$ and $x_{2} f_{2} x_{4}$ ' and ' $x_{5} g_{1}$ '. We say that a sequence assigning Barack Obama to $x_{5}$ and the property of being president to $g_{1}$ satisfies the latter sequence just because, as it so happen, Barack Obama is president. ${ }^{18}$ Similarly, a sequence satisfies the former sentential function if it assigns appropriate denotations to the variables. One way to do so would be to assign Aristotle, Lee Harvey Oswald, Alexander, and J.F.K to $x_{1}, x_{2}, x_{3}$, and $x_{4}$, respectively, as well as the relations expressed by ' $x$ is a

\footnotetext{
${ }^{17}$ I would like to stress that there are other ways of developing the technical details than the ones I present here. The philosophical import, as it relates to Tarski's definition of logical consequence, remains the same. Technically, a sequence is just a total function on the natural numbers (to objects of the appropriate kind). We should note though, that there are no requirements of this function being injective or surjective. A sequence may map all natural numbers to the same object and even though an object is of the appropriate kind for a given sequence it need not obtain in that sequence, i.e., be the image of any natural number. Let $f$ be such a total function on the natural numbers. An alternative way of denoting $f$, which we use in the text, is as $\langle f(1), f(2), \ldots, f(n), \ldots\rangle$.

18 There are some delicate issues in the vicinity here, which we will mostly ignore. One question that may concern us is what kind of object a predicate designates - are we required to be realists about properties such that there is a an object that the predicate '... is red' names? I will not pursue a discussion of these issues here. Suffice it to note that predicates are the kind of things that have extensions. A sequence, then, can simply assign a a set of objects to the 1-place predicate variables of the language, and a set of tuples to the relational predicates of the language, and so on. And it can do so whether or not extensions are in fact mediated by such a thing as the property, an existing object in itself. See Etchemendy [1990: ch. 3-4] for some comments on this issue and other issues related to the notion of satisfaction which I skip over here.
} 
teacher of $y$ ' and ' $x$ killed $y$ ' to $f_{1}$ and $f_{2}$, respectively. ${ }^{19,20}$

Using the notion of satisfaction, Tarski defines first a model for a class of sentences, $L$, and then the notion of $X$ following logically from a class of sentences $K$ :

Let $L$ be any class of sentences. We replace all extra-logical constants which occur in the sentences belonging to $L$ by corresponding variables, like constants being replaced by like variables, and unlike by unlike. In this way we obtain a class $L^{\prime}$ of sentential functions. An arbitrary sequence of objects which satisfies every sentential function of the class $L^{\prime}$ will be called a model or realization of the class $L$ of sentences (in just the sense one usually speaks of models of an axiom system of a deductive theory). If, in particular, the class $L$ consists of a single sentence $X$, we shall also call the model of the class $L$ the model of the sentence $X$.

In terms of these concepts we can define the concept of logical consequence

\footnotetext{
${ }^{19}$ It is clear that the relation expressed by ' $x$ killed $y$ ' is not the same as the relation expressed by ' $x$ was killed by $y$ ' even though both involve the same relation proper, that of one entity killing another entity. Order matters, which is why we demand that the extension of a relational predicate is a set of tuples. We therefore also designate the relation by ' $x$ killed $y$ ' instead of merely '... killed ...' since one occurrence of '...' is indistinguishable from the other.

${ }^{20}$ As the reader may have noticed our notion of a sequence as it was defined in note 17 is no longer sufficient. Since we have different objects in the satisfaction domain of different variables we will need to extend the notion of satisfaction of a sentential function by a sequence. If the language in question contains $n$ different semantical categories, we simply view satisfaction not as a two-termed relation between a sequence and a sentential function, but as an $n+1$-ary relation holding between $n$ sequences, one for each semantical category, and the sentential function in question. The $n$ distinct sequences now satisfy the sentential function if and only if the the objects assigned to the name-variables are in the various extensions assigned to the predicate-variables, the relation-variables, and so on. From this picture it is easy to regain a notion of a single sequence which can satisfy a sentential function. We simply view each sequence properly as a sequence of $n$ distinct sequences, one for each semantical category. Satisfaction can now once again be defined as a two-termed relation between a sequence, so defined, and a single sentential function. Tarski calls this the method of many-rowed sequences (Tarski [1956b: 227-230]). There are other ways to solve this issue. See Tarski [1956b: 228-231] for a discussion of what Tarski calls the method of semantical unification of the variables. The idea here is to make the satisfaction domain, for variables of all types, consist of relations, and to define appropriate one-to-one correspondences between these relations and objects of the appropriate type for a given variable.
} 
as follows:

The sentence $X$ follows logically from the sentences of the class $K$ if and only if every model of the class $K$ is also a model of the sentence $X$. [1956a: 417]

A sequence of objects which satisfies every sentential function obtainable from the class of sentences $L$ is, in Tarski's terminology, a model of the class of sentences $L$. Using this notion of model Tarski gives a precise definition of logical consequence: $X$ follows logically from $K$ if and only if every model of $K$ is a model of $X$.

It should be clear that the extension of Tarski's defined concept of logical consequence will be relative to the division of the language into logical and non-logical terms. We can see this reflected directly in the definition by the fact that what sequences are models of what classes of sentences will depend on this division.

Underlying our whole construction is the division of all terms of the language discussed into logical and extra-logical. This division is certainly not quite arbitrary. If, for example, we were to include among the extra-logical signs the implication sign, or the universal quantifier, then our definition of the concept of consequence would lead to results which obviously contradict ordinary usage. On the other hand, no objective grounds are known to me which permit us to draw a sharp boundary between the two groups of terms. It seems possible to include among the logical terms some which are usually regarded by logicians as extra-logical without running into consequences which stand in sharp contrast to ordinary usage. [1956a: 418-419]

Tarski is here claiming both that the division between logical and non-logical cannot be wholly arbitrary, but at the same time, that he knows of no way to draw a principled 
distinction between the two. Tarski would later suggest a principled way of demarcating the logical terms from the non-logical terms (Tarski [1986]). Tarski's idea is to view those terms as logical that are invariant under permutations of the underlying domain. What it means for a term to be invariant under permutations of the underlying domain is that the truth value of sentences involving this term remains stable when the objects of the domain are permuted. For example, under this conception, 'was an American president' cannot be treated as a logical term. For while it is true that Abraham Lincoln was an American president it is untrue that Benjamin Franklin was an American president. Hence, the true statement 'Abraham Lincoln was an American president' becomes false under the permutation of the domain which maps the designation of 'Abraham Lincoln', that is, Abraham Lincoln, to Benjamin Franklin.

\subsubsection{Adequacy of The Definition}

Immediately after presenting his definition of logical consequence Tarski states:

It seems to me that everyone who understands the content of the above definition must admit that it agrees quite well with common usage. This becomes still clearer from its various consequences. In particular, it can be proved, on the basis of this definition, that every consequence of true sentences must be true, and also that the consequence relation which holds between given sentences is completely independent of the sense of the extralogical constants which occur in these sentences. In brief, it can be shown that the condition $(\mathrm{F})$ formulated above is necessary if $X$ is to follow from the sentences of the class $K$. [1956a: 417]

We see Tarski claiming, on behalf of his definition, that:

(i) It can be proved that every consequence of true sentences must be true. 
(ii) The relation of consequence is independent of the meaning of the non-logical constants involved.

(iii) Showing (i) and (ii) amounts to proving that condition (F) is a necessary condition for logical consequence to obtain.

The last claim, (iii), merely reflects that Tarski takes the content of condition (F) to be given by (i) and (ii). Variants of (i) and (ii) are standardly seen to be intimately linked with the notion of logical consequence and are consequently being discussed by the majority of theorists working on logical consequence. ${ }^{21}$ The content of (i) is often expressed in what we call 'the tenet of necessity':

\section{(Necessity)}

If an argument is logically valid, then the conclusion follows necessarily from the premises. That is, it is not possible for the conclusion to be false while the premises are true.

The exact formulation and content given to Necessity differs from author to author, but the gist remains: in a logically valid argument there is a modal connection between premises and conclusion. The conclusion follows of necessity if the argument is valid. There are, of course, various ways of making sense of the notion of necessity that is involved here, but it is almost universally accepted that there must be such a connection between premises and conclusion in a valid argument. ${ }^{22}$

\footnotetext{
${ }^{21}$ I am not hereby claiming that the majority of theorists accept the content of the tenets to be discussed below, though most do in some form or other. See, for example, McKeon [2010], Beall \& Restall [2006], Sher [1996], Shapiro [1998]. There is of course divergence in how the exact content of (i) and (ii) is spelled out, and often more conditions are put on the relation of consequence; for example that it should be knowable a priori (if it can be known at all) that it obtains (Hanson [1997: 378]). Logical consequence is also thought to be normative: you are somehow doing something wrong if you accept the premises of a valid argument, yet deny its conclusion (Beall \& Restall [2006: 16]).

${ }^{22}$ I know of no one who affirmatively denies that there should be a modal connection between premises and conclusion in a valid argument. However, there are those who feel that a debate as to why we take Necessity to be essential to logical consequence is needed (Smiley [1998: 602]). See section 1.2.3.
} 
It is often claimed that logical consequence is not just necessary truth-preservation, but a special case of the latter. Often it is expressed by saying that logical consequence is necessary truth-preservation in virtue of form. This, which we may call 'the tenet of formality', is often given in the following way:

\section{(Formality)}

Whether an argument is logically valid is fully determined by its form. An alternative formulation is that an argument is logically valid if and only if all other arguments with the same form are logically valid.

Now, it is not clear exactly what is meant by form in the above. Standardly, the form of an argument is taken to be a function of the forms of the sentences involved in the argument, and the form of a sentence is given by the distribution of logical and nonlogical terms in the sentence. ${ }^{23}$ The acceptance of Formality, therefore, is relative to a distinction between logical and non-logical terms. It should be clear that the phrasing of Formality, as it is presented above, is not identical to the phrasing which Tarski gives to one of the consequences of his definition ((ii) above). Nevertheless, Formality is often presented as a way to make the content of (ii) clear. If the validity of an argument is truly independent of the meanings of the non-logical terms, then the exact designation of those terms will not matter, and all arguments with that form will be valid. Similarly, if all arguments of a given form are valid, then it follows that, the meanings of the non-logical terms does not matter for this validity to come about. All

\footnotetext{
${ }^{23}$ It is sometimes said that the logical form of a sentence is determined solely by the distribution of logical constants throughout the sentence in question. But this would not be quite right insofar as we want logical validity to be fully determined by the form of the involved sentences. Consider 'if Kasper is happy, then Kasper is happy' and 'if Kasper is happy, then Kasper is sleeping'. (Or, their corresponding formal counterparts in a first-order language, $F a \rightarrow F a$ and $F a \rightarrow H a$ ). Obviously, if form is determined only by the arrangement and distributions of logical terms/constants, then these two have the same logical form - assuming the only logical term (constant) to be 'if... then...' $(\rightarrow$ ). But, one is a logical truth, the other not, and we have a counterexample to Formality. Form, in the sense in which it determines the validity of arguments, is a function of the distribution of both logical and non-logical terms in the involved sentences. This point is also made by Sagi [2013: 108, n.3].
} 
of this, of course, is modulo the assumption that the expressiveness of the non-logical part of the language is not a factor in deciding validity. ${ }^{24}$

How does Necessity relate to Tarski's claim that consequences of true sentences must be true? The matter here is contentious, since (i) has two readings, one obviously true, the other not. Unfortunately Tarski never produces the proof which he alludes to in the formulation "it can be proved, on the basis of this definition, that every consequence of true sentences must be true" [1956a: 417], so we cannot immediately tell which reading corresponds to the result Tarski thought could be proved. On one reading, the 'must' in Tarski's formulation serves to indicate that an argument cannot be valid unless it has either a true conclusion or at least one false premise. This can be shown, and the proof is straightforward. Suppose the argument from $K$ to $X$ is valid on Tarski's account, but that $K$ consists of only true sentences while $X$ is false. Let $K^{\prime}$ and $X^{\prime}$ be the sentential functions obtainable from $K$ and $X$ by replacing the non-logical constants by appropriate variables. Consider the sequence which assigns to the variables of $K^{\prime}$ and $X^{\prime}$ the objects which were denoted by the terms these variables replaced. It is immediate that this sequence is a model of $K$ but not of $X$, contradicting that $X$ follows from $K$. Hence it cannot be the case that $X$ follows from $K, K$ consists of only true sentences, and $X$ is false. Accordingly, if $X$ follows from $K$ at least one of $K$ is false or $X$ is true.

But if this is the reading which we give to (i), it does not capture what is given in Necessity. According to Necessity there must be a genuine modal connection between the premises and conclusion in a valid argument. This is the second reading of 'must' in (i), and as we will discuss later there is nothing in Tarski's definition of consequence which secures that this reading of (i), under which it is equivalent to Necessity, is true.

\footnotetext{
${ }^{24}$ See Sagi [2013: 20ff] for a discussion of the content of both Necessity and Formality as these relate to logical consequence.
} 


\subsubsection{Interpretational vs. Representational Semantics}

To motivate Etchemendy's crucial distinction between what he calls interpretational and representational semantics we will look at the simplest case of models: models for a propositional language. ${ }^{25}$ A standard propositional language consists of a stock of sentence letters (i.e., $p, q, r$, and so on). We will sometimes refer to these also as atomic sentences, atomic propositions, or propositional variables, and we denote the class of all of these by $P$. Besides those we have the standard truth-functional connectives (i.e., $\wedge, \neg, \vee$, and $\rightarrow$ ). Our language consists of the following wffs:

$\left(\mathrm{at}_{p}\right)$ All members of $P$ are wffs.

$(\neg p)$ If $\alpha$ is a wff, $\neg \alpha$ is a wff.

$\left(\wedge_{p}\right)$ If $\alpha$ and $\beta$ are both wffs, $\alpha \wedge \beta$ is a wff.

$\left(\vee_{p}\right)$ If $\alpha$ and $\beta$ are both wffs, $\alpha \vee \beta$ is a wff.

$\left(\rightarrow_{p}\right)$ If $\alpha$ and $\beta$ are both wffs, $\alpha \rightarrow \beta$ is a wff.

$\left(\mathrm{T}_{p}\right)$ Nothing is a wff unless constructed by a finite application of the above rules.

A model, for our language, is a total function on the class of propositional variables, $P$, into the set $\{0,1\} .{ }^{26}$ That is:

\footnotetext{
${ }^{25}$ It is not really common to speak of models in the case of propositional languages. Rather, terms such as valuations, interpretations, and sometimes just truth-tables are used, though it should be noted that a row in a truth-table is not a model unless every atomic sentence of the language is represented. We will sometimes follow suit with this usage, though it should always be clear from context what kind of model, interpretation, or valuation we are talking about.

${ }^{26} 1$ and 0 are meant to represent the values true and false, respectively. For this purpose it does not really matter what exact entities are assigned to the sentence letters, and sometimes we will speak as if they are assigned actual truth values; what matters is that we have two distinct entities along with a designation of which represents true and which represents false. It is common to work with 0 as representing false and 1 as representing true, though there are authors who break with this tradition. See for example Chihara [1998: 198], who uses 0 as the designated value representing true and 1 as representing false.
} 
$\left(\operatorname{model}_{p}\right) m: P \mapsto\{0,1\}$.

We define truth in a model, $m$, of a formula, $\alpha$, which we write as $m \Vdash \alpha$, in the standard recursive manner. We use $m \forall \alpha$ to denote that it is not the case that $m \Vdash \alpha$.

$\left(\mathrm{at}_{p}\right)$ For all sentence letters, $p: m \Vdash p$ if and only if $m(p)=1$.

$(\neg p) m \Vdash \neg \alpha$ if and only if $m \Vdash \alpha$.

$\left(\wedge_{p}\right) m \Vdash \alpha \wedge \beta$ if and only if $m \Vdash \alpha$ and $m \Vdash \beta$.

$\left(\vee_{p}\right) m \Vdash \alpha \vee \beta$ if and only if $m \Vdash \alpha$ or $m \Vdash \beta$.

$\left(\rightarrow_{p}\right) m \Vdash \alpha \rightarrow \beta$ if and only if $m \Vdash \alpha$ or $m \Vdash \beta$.

It is easy to verify that for each and every wff, $\alpha$, of the language, and each model, $m$, we will have $m \Vdash \alpha$ or $m \Vdash \alpha$.

Etchemendy motivates his distinction between representational and interpretational model-theoretic semantics by pointing out that there are two parameters to which a given sentence owes its truth: The language and the world. Language is responsible for a given sentence having the meaning that it does; that is, for expressing the claim that it does. But the world is responsible for it being true, by being as the sentence claims. Consider 'Snow is white'.

It is due to the language that the sentence means what it means, that it makes the claim it does. But it is due to the world that snow is white. Appropriate changes on either side would have made our atomic sentence ['Snow is white'] false. Thus, had the language been somewhat different, this sentence would have been false in spite of the whiteness of snow-say, if 'white' had meant hot. On the other hand, had the world been different, 
this sentence might have been false in spite of its meaning - say, had snow been red. (Etchemendy [1990: 18])

If we view our model theory interpretationally, differences in models represent differences in the first parameter: language. If, on the other hand, we view our model theory representationally, we see differences between models as differences in the second parameter: the world. We can capture the two approaches in slogan form.

(int) Interpretational model-theoretic semantics: Each model represents a different interpretation that the non-logical terminology could have had affecting the truth values of the sentences of our language.

(rep) Representational model-theoretic semantics: Each model represents a different way that the world could have been affecting the truth values of the sentences of our language.

This puts us in a position to say something informative about Davidson's puzzle. ${ }^{27}$ The puzzle is that truth in a model is a relation $-m \Vdash \alpha$ means that a certain relation holds between $m$ and $\alpha$-whereas "truth, perhaps the preeminent semantic concept, does not relate a sentence to something else; it simply applies or fails to apply, so to speak, absolutely" (Etchemendy [1990: 12]).

[I]t is worthwhile taking seriously Davidson's simple, initial point: truth $i s$, after all, a property; truth in a model, a relation. What bearing can a characterization of such relational concept have on our ordinary "monadic" concept of truth? If there is no close tie between the two, as Davidson occasionally implies, then why is the relation of "truth in a model" given a name that sounds so misleading? [1990: 13]

\footnotetext{
${ }^{27}$ Davidson [1973]. Etchemendy uses this puzzle to motivate his distinction. See Etchemendy [1990: ch. 2].
} 
What we are pointing out in (int) and (rep) are the two ways in which truth in a model can be related to our monadic truth predicate, which expresses the property of truth, as Etchemendy puts it. On the former view truth in a model is truth (i.e., our ordinary monadic notion of truth) under a particular interpretation of the language. On the latter view, truth in a model is truth (i.e., our ordinary monadic notion of truth) in a particular circumstance. From the perspective of (int), the reason that truth in a model can sometimes give a different verdict than our ordinary monadic truth predicate, as used by us, is that not all interpretations of the language coincide with the one our language actually enjoys. From the perspective of (rep), the two come apart since not all circumstances are identical to the circumstances as they actually are. The "right" model-sometimes called the intended model - is the model which represents truth under the actual interpretation of the language and truth in the actual circumstance, respectively. In a sense, the setup of the model theory serves to deny that there is anything special about the ordinary monadic concept of truth when it comes to logical consequence. Sure, we live in this world and we speak our language, but that could certainly have been different in which case our ordinary monadic truth-predicate would apply to statements other than what it actually does. ${ }^{28}$

As the reader might have noticed, the way we have briefly characterized the two approaches here, they do not seem to be mutually exclusive. If the model theory can reflect changes in one of the two parameters, then it should be able to reflect changes in both. ${ }^{29}$ The way in which we have been using the term interpretational so far, we take it to be committed to the claim that each model represents the actual world with a different interpretation of the non-logical terminology. We shall later separate the two claims involved here: that each model represents the actual world and

\footnotetext{
${ }^{28}$ Of course, from the interpretational perspective not all parts of the language could have had a different interpretation, as the interpretations of the logical terms remain fixed throughout the models.

${ }^{29}$ In chapter 3 we will discuss how a model can both represent a non-actual circumstance as well as provide an interpretation for the non-logical terms.
} 
that each model provides an interpretation of the non-logical terminology. For now we will continue to speak of interpretational semantics as being committed to both claims.

To get clearer on the difference between the two approaches it is instructive to look at models which are obviously not the intended model, models where truth in a model and our ordinary monadic truth predicate come apart. As Etchemendy points out the (non-intended) models of our model theory represent vastly different information depending on whether we view them representationally or interpretationally [1990: 18ff]. Consider the sentence letter $p$ as the formal counterpart of the English sentence 'Snow is white'. Let $m^{\prime}$ be a model such that $m^{\prime}(p)=0$. This model, then, represents our sentence 'Snow is white' to be false, and it is consequently not the intended model in the sense we discussed above, since snow is in fact white and this is what the sentence expresses. From the clauses $\left(\vee_{p}\right)$ and $(\neg p)$ set out above we can see that $m^{\prime} \Vdash p \vee \neg p$ and that in accordance with the antecedently given meaning to $p, m^{\prime}$ represents that the sentence 'Snow is white or snow is not white' is true. From the interpretational point of view, the model $\mathrm{m}^{\prime}$ supports the following counterfactual:

(ci) 'Snow is white or snow is not white' would still be true in the actual world had the constituent sentence 'Snow is white' meant something that is false, perhaps that George Washington had a beard.

From the representational perspective we have that $m^{\prime}$ supports the counterfactual:

(cii) 'Snow is white or snow is not white' would still be true with its actual meaning had the world been such that snow was not white, perhaps it was black.

These counterfactuals provide radically different information. In (ci) the state of the world remains fixed and we get information about what would have happened to the 
sentence 'Snow is white or snow is not white' under the assumption that the constituent sentence 'Snow is white' had a different meaning. In (cii), on the other hand, the meaning of the sentence 'Snow is white or snow is not white' remains fixed and we get information about what would have happened to the truth value of the sentence under the assumption that snow was in fact not white. Of course, both (ci) and (cii) could be captured by the following counterfactual:

(ciii) 'Snow is white or snow is not white' would still be true had 'Snow is white' been false.

But this should not lure us into thinking that the two approaches here are the same. Though (ciii) can be seen as capturing the meaning of both (ci) and (cii), in the sense of being entailed by both, what (ciii) does not mention explicitly is that there are two radically different ways in which 'Snow is white' can be false. One way is due to language, it could simply mean something which is false. The other is due to the world, snow could have been some color other than white.

We may now say a little about the intended model, the model that is meant to connect our notion of the ordinary monadic truth predicate and the notion of truth in a model. From the perspective of interpretational semantics the intended model is the one where the meaning-parameter takes on a value that coincides with its actual value for all sentences of the language. That is, the model in which the non-logical terminology receives its actual interpretation. To use our example from above, the right model, when $p$ is interpreted to have the meaning of 'Snow is white', is a model such that $p$ is assigned the value 1. Only in such a model does the meaning-parameter coincide with its actual value given that snow is actually white. Similarly for other sentences of the language: suppose we have an antecedently interpreted formal language consisting of the sentence letters $p_{1}, \ldots, p_{n}, \ldots$. We may note that in order for there to be an intended 
model, our formal language must have an antecedently given interpretation which the intended model then respects. If the atomic sentences have no prior interpretation associated with them the question of their actual interpretation, and so the intended model, does not even arise. The intended model, call it $m^{+}$, is defined as follows:

$m^{+}\left(p_{i}\right)=1$ if, given the antecedently interpretation given to $p_{i}$, it means something that is actually true.

$m^{+}\left(p_{i}\right)=0$ if, given the antecedently interpretation given to $p_{i}$, it means something that is actually false.

This model, $\mathrm{m}^{+}$, is often referred to, from an interpretational perspective, as the intended interpretation. ${ }^{30}$

From a representational perspective, the intended model is the one in which the world-parameter takes on a value that coincides with its actual value. In other words, the right model is the one that represents the world to be as it actually is. Assuming the same antecedently given interpretation to the sentence letters, the intended model from this perspective is therefore also given by $m^{+}$above. This is so because if $p_{i}$ has an antecedently given interpretation under which it is true in the actual world, a model can represent the actual world only if it makes $p_{i}$ true. In the case of interpretational semantics we noted that the notion of an intended model makes sense only relative to an antecedently given interpretation. We may now note that this conclusion is not forced upon us in the case of representational semantics. The intended model, as we defined it for representational semantics, is the one where the world-parameter takes on its actual value. In other words, it is the model representing the actual world. If there is a way for models to represent independently of the truth values given to the sentences of the language, then we could have an intended model without having an

\footnotetext{
30 The reader may notice that we are assuming that the antecedently given interpretation given to the sentence letters makes each of them have exactly one of the two truth values, true or false.
} 
antecedently interpreted language. These issues will concern us at great length when discussing languages for which the models of the model theory include more structure than merely assigning a truth value, such as first-order models.

\subsubsection{Interpretational Model-Theoretic Semantics}

A model, in the contemporary sense, also sometimes called a structure or an interpretation, for a language is an ordered pair, $\langle D, I\rangle$, where $D$ is a non-empty set, and $I$ is a function that assigns appropriate denotations to the non-logical constants of the language.

Let us consider a simple monadic first-order language, $L$, containing the truthfunctional connectives, $\neg$ and $\wedge$, and the quantifier $\forall$, as logical constants. ${ }^{31}$ As nonlogical constants we will have a stock of 1-place predicates, $F, G$, and so on, a stock of individual constants (names), $a, b$, and so on, and a stock of individual variables, $x, y$, and so on. We will refer to a variable or an individual constant as a term, and use $t_{1}$, $t_{2}, t_{3}, \ldots$, (with or without subscript) to range over terms. The wffs of the language are the standard ones given by the following.

(at) If $t$ is a term and $F$ a 1 -place predicate, $F t$ is a wff.

$(\neg)$ If $\alpha$ is a wff, $\neg \alpha$ is a wff.

$(\wedge)$ If $\alpha$ and $\beta$ are both wffs, $\alpha \wedge \beta$ is a wff.

$(\forall)$ If $x$ is a variable and $\alpha$ is a wff, $\forall x \alpha$ is a wff.

(T) Nothing is a wff unless constructed by a finite application of the above rules.

\footnotetext{
31 There is of course nothing in principle privileged about first-order languages when it comes to Tarski's or the contemporary model-theoretic definitions of logical consequence. We are using this language simply to explain the connection between the two conceptions. For this reason we consider only a monadic language without identity. It should be fairly obvious to extend the relation to languages containing more complex expressions.
} 
We can now be more explicit about the function $I$. Let $\langle D, I\rangle$ be a model. Then $D$ is a non-empty set. $I$ is a function such that for each predicate, $F, I(F) \subseteq D$ and for each individual constant, $a, I(a) \in D$. We define the satisfaction of a formula by a model relative to an assignment function. An assignment function, $v$, relative to $\langle D, I\rangle$, is a function from the variables of $L$ into $D$, such that each variable is assigned a unique member of $D$. If $o$ is an object in $D$ we define $v[x / o]$ to be the assignment function which is just like $v$ except it maps the variable $x$ to the object $o{ }^{32}$ Let $\operatorname{den}(t)_{M, v}=I(t)$, if $t$ is an individual constant and $\operatorname{den}(t)_{M, v}=v(t)$, if $t$ is a variable. We now define the satisfaction of a formula, $\alpha$, in a model, $M$, relative to an assignment function, $v$-which we write $M \Vdash_{v} \alpha$.

(at) $M \Vdash_{v} F t$ if and only if $\operatorname{den}(t)_{M, v} \in I(F)$.

$(\neg) M \Vdash_{v} \neg \alpha$ if and only if it is not the case that $M \Vdash_{v} \alpha$.

$(\wedge) M \Vdash_{v} \alpha \wedge \beta$ if and only if $M \Vdash_{v} \alpha$ and $M \Vdash_{v} \beta$.

( $\forall) M \Vdash_{v} \forall x \alpha$ if and only if $M \Vdash_{v[x / o]} \alpha$, for all $o \in D$.

We say that a formula $\alpha$ is true in a model $M$, and write $M \Vdash \alpha$, if and only if $M \Vdash_{v} \alpha$, for all assignment functions $v$ relative to $M=\langle D, I\rangle$.

The meanings of the logical constants are decided without reference to any particular model and are given by the general clauses above. This mirrors the effect, on Tarski's account, of not replacing the logical terminology with variables to obtain the sentential functions of interest. But instead of replacing each occurrence of a nonlogical term in $X$ with a variable, to find if a given sequence satisfies the corresponding sentential function $X^{\prime}$, we now assign objects directly to the non-logical terms of $X{ }^{33}$

\footnotetext{
${ }^{32}$ Technically, $v[x / o](y)=v(y)$, for all $y \neq x$ and $v[x / o](x)=o$.

${ }^{33}$ See Etchemendy [1990: ch. 4] for a discussion of the relationship between Tarski's original account of consequence, as given in Tarski [1956a], and the contemporary model-theoretic account.
} 
This picture does not change in any significant way when we consider languages with other logical constants and non-logical constants. The truth clauses for the logical constants are given independently of the models, in the sense that they make reference to no particular model, and when we add non-logical constants we simply extend the definition of $I$ so that it assigns appropriate denotations to these categories as well. For example, adding a 2-place relation, say $R$, to the language, we demand that $I(R) \subseteq D \times D$. And in general, for any $n$-ary predicate, $P$, treated as a non-logical constant, we have $I(P) \subseteq D^{n}$. If we choose to add quantifiers to our language which quantify over predicates, we once again add variables of appropriate type to the language itself, and will have to extend the notion of an assignment function accordingly.

From an interpretational perspective the idea behind the model theory is that the models give us interpretations of the non-logical terminology in the actual world. Consider the formula $F a$ with the antecedently given interpretation of 'Snow is white'. A model, $M=\langle D, I\rangle$, such that $M \Vdash F a$ (or equivalently, $M \Vdash \neg F a$ ), still supports the counterfactual given above in (ci), but there is now more to say about the way in which it does so. $F a$ is false in $M$ because $I(a) \notin I(F)$, as in accordance with the clauses set forth in section 1.1.3. This means that $F$ is interpreted, by the model, to mean something such that the extension of $F$ is $I(F)$, while $a$ is interpreted to mean something such that it designates the object $I(a)$, and since the former does not include the latter the sentence is false in that particular model.

We have the following tenet of an interpretational approach to model-theoretic semantics:

(INT) A model, $M=\langle D, I\rangle$, interprets the non-logical term $\eta$ to have the extension $I(\eta)$. 
Here, $\eta$ is being used as a variable ranging over the categories of non-logical expressions in the language. If $\eta$ is a name, for example, then $I(\eta)$ will be an object of the domain, meaning that $\eta$ is interpreted as a name for $I(\eta)$; if $\eta$ is an n-ary predicate, then $I(\eta)$ will be a set of n-tuples of objects, and so on.

An interpretational model theory, as we have been using the term so far, is not only committed to the view that models interpret, but also to the view that each model represents the actual world.

(MRA) All models represent the actual world. If $\alpha$ is true in a model this represents that $\alpha$ is actually true under the interpretation given to it by that model.

When we give our standard definition of logical consequence as truth-preservation in all models seen in accordance with (INT) and (MRA), Etchemendy claims, we are stating Tarski's definition of logical consequence. And on the basis of this, Etchemendy launches his criticism of the latter. There are therefore two essential claims involved.

(claim1) Tarski's original definition of logical consequence gives rise to a standard modeltheoretic definition which sees the underlying models in accordance with (INT) and (MRA).

(claim2) A model-theoretic definition of logical consequence which sees the underlying models in accordance with (INT) and (MRA) is fundamentally mistaken.

The first claim is of utmost historical importance and many commentators have criticized Etchemendy for being mistaken on this account. ${ }^{34}$ What matters for the truth of the first claim is exactly what Tarski meant by his definition of logical consequence given in Tarski [1956a]. For our purposes, the truth or falsity of the second claim takes center stage. That is not to say that there is nothing interesting about the first claim,

\footnotetext{
${ }^{34}$ A prominent example being Gómez-Torrente [1996].
} 
but merely to state that our aim, in this thesis, is another. That said, the two claims cannot so easily be separated in the replies to Etchemendy's original criticism and we will consequently have something to say about both.

\subsubsection{Is Tarski's Account Interpretational?}

Let us start by saying something about the first claim: that Tarski's original definition of logical consequence gives rise to a standard model-theoretic definition which sees the models in accordance with (INT) and (MRA).

On Tarski's original approach, a sentence $X$ is a logical truth when all sequences are models of $X$-it is the limiting case of logical consequence: a sentence which follows from all classes of sentences. Demanding that all sequences are models of $X$ is, in effect, to demand that the universal closure of the sentential function $X^{\prime}$ is true. Suppose $x_{1}^{1}, \ldots, x_{k}^{1}, x_{1}^{2}, \ldots, x_{l}^{2}, \ldots, x_{1}^{n}, \ldots, x_{m}^{n}$ are the variables of appropriate type present in the sentential function $X^{\prime}{ }^{35}$ If all sequences are models of $X^{\prime}$, we know that for all combinations of appropriate values of the variables in $X^{\prime}$, the result is true. That is, $\forall x_{1}^{1}, \ldots, x_{k}^{1}, \ldots, x_{1}^{n}, \ldots, x_{m}^{n} X^{\prime}$ is true if all sequences are models of $X$. Conversely, since for the case at hand it clearly does not matter what objects a sequence assigns to variables that are not present in $X^{\prime}$, we get that $\forall x_{1}^{1}, \ldots, x_{k}^{1}, \ldots, x_{1}^{n}, \ldots, x_{m}^{n} X^{\prime}$ is true only if all sequences are models of $X$. We can thus see that the logical truth of $X$, on Tarski's approach, reduces to the actual truth of the universal closure of the sentential function $X^{\prime}$

The result extends to logical consequence. Let $x_{1}^{1}, \ldots, x_{k}^{1}, \ldots, x_{1}^{n}, \ldots, x_{m}^{n}$ be variables ranging over the appropriate semantic categories which have replaced appropriate expressions in $K$ and $X$ to form the sentential functions $X^{\prime}$ and (finite) class of sentential

\footnotetext{
${ }^{35}$ We can be sure that there are only finitely many such variables, insofar as our language only allows finite formulae.
} 
functions $K^{\prime}{ }^{36} X$ is then a logical consequence of $K$, on Tarski's account, if and only if the following is true:

$$
\text { (*) } \forall x_{1}^{1}, \ldots, x_{k}^{1}, \ldots, x_{1}^{n}, \ldots, x_{m}^{n}\left(\left(\bigwedge_{k^{\prime} \in K^{\prime}} k^{\prime}\right) \rightarrow X^{\prime}\right) \text {. }
$$

We need to convince ourselves that $\left(^{*}\right)$ is true if and only if for all models $M$, seen in accordance with (INT) and (MRA), such that $M \Vdash \Gamma$, we also have $M \Vdash \alpha$, where $\Gamma$ and $\alpha$ are the formal language counterparts of the sentential function $K^{\prime}$ and $X^{\prime}{ }^{37}$ We may note the following: If for all $M$ s.t. $M \Vdash \Gamma$, we have $M \Vdash \alpha$, it follows that no matter what actual objects are assigned as denotations for the non-logical terminology in $\Gamma$ and $\alpha$, if all of $\Gamma$ are true, so is $\alpha$. But this is just to say that all assignments of objects to the variables of $K^{\prime}$ and $X^{\prime}$ make $\left(^{*}\right)$ true, since the logical constants in $\Gamma$ and $\alpha$ are assumed to enjoy the same interpretation as the logical terms in $K$ and $X$. Conversely, if there is a model making all of $\Gamma$ true, but $\alpha$ false, it is easy to see that there will equally be an assignment to the variables of $K^{\prime}$ and $X^{\prime}$ making $\left(\bigwedge_{k^{\prime} \in K^{\prime}} k^{\prime}\right) \rightarrow X^{\prime}$ false. Hence $\left(^{*}\right)$ is false. We can therefore see how Tarski's account, when extended to a contemporary model-theoretic account, endorses (INT) and (MRA).

Now, this conclusion is not an uncontroversial matter, and it is disputed by a score of authors. ${ }^{38}$ The crucial assumption, of course, is that the sequences serve only to assign appropriate denotations to (i.e., interpret) the variables in the sentential functions, and that they are not meant to serve any role in representing alternative circumstances. Although, as mentioned, my primary focus is not to support the claim that Tarski's account gives rise to a model theory seeing the models in accordance with (INT) and (MRA), I should like to mention a few considerations that seem to point in

\footnotetext{
${ }^{36}$ We assume that $K^{\prime}$ is finite to make sense of the conjunction of the sentences. Of course, the picture generalizes to the infinite case, but we would then need to allow for infinite conjunctions.

${ }^{37} M \Vdash \Gamma$ is short for $M \Vdash \gamma$ for all $\gamma \in \Gamma$.

38 Most prominently, perhaps, Gómez-Torrente [1996, 1998] and Sher [1996].
} 
this direction.

When Tarski rejects the idea of holding condition $(\mathrm{F})$ a sufficient condition for logical consequence, he explicitly states that the reason is that "[t]his condition may in fact be satisfied only because the language with which we are dealing does not possess a sufficient stock of extra-logical constants" (Tarski [1956a: 415-416]). Now, we may note that such considerations, as to whether our language possesses enough extra-logical constants, ought to be superfluous if Tarski intended his definition to be of a representational nature. Take for example our above argument from the sentence 'Abraham Lincoln had a beard' to 'Abraham Lincoln was an American President'. From a representational perspective we would not need to consider whether we had a name in our language which was able to refer to a person who has a beard but was not in fact an American President. The only consideration that would matter would be whether it is indeed possible for Abraham Lincoln to have a beard without being an American President. ${ }^{39}$

Secondly, it is hard to reconcile the fact that Tarski explicitly states that his notion of logical consequence coincides with material consequence when all terms are treated as logical constants with the fact that Tarski should have seen himself as giving a kind of representational theory. If the definition was somehow involved with representations of different circumstances, then the former claim would not be true. To see this we need only note that there are countless arguments which are actually truth-preserving (i.e., are instances of material consequence) though are not truth-preserving in all possible circumstances. For example, the simple argument:

\footnotetext{
${ }^{39}$ In fairness to the defenders of Tarski intending something different, the above quote continues: "The condition (F) could be regarded as sufficient for the sentence $X$ to follow from the class of sentences $K$ only if the designations of all possible objects occurred in the language in question" [1956a: 416, my emphasis]. Tarski does state that every possible object should be able to be designated. Of course, it is a contentious matter exactly what the word 'possible' serves to indicate in this sentence. I submit that it is somewhat of a step to claim that Tarski is claiming that the designation of a merely possible Abraham Lincoln who has a beard but is not an American President should be in the language.
} 
- Copenhagen is the capital of Denmark.

- Therefore, Wellington is the capital of New Zealand.

This argument is surely truth-preserving, hence the conclusion is a material consequence of the premise. But it is possible that Wellington had not been the capital of New Zealand while the capital of Denmark still was Copenhagen. This argument would be logically valid on Tarski's account if all terms are treated as logical constants, which is hard to reconcile with there being a representational aspect to Tarski's account.

Now, none of these considerations are knock-down arguments against different readings of Tarski's original definition, and they are not meant to be. We will have more to say about this when discussing some of the replies to Etchemendy. For now, what we need to establish is that there is a fairly intuitive reading of Tarski in which the model theory which his definition gives rise to views the models in accordance with (INT) and (MRA). I hope to have established as much.

One way to object to the last two considerations would be to point out that they turn on a tacit claim, the truth of which has not been established. The claim being that if the model theory is not representational, then it must see the models in accordance with (INT) and (MRA). Let us assume that there are only two parameters to which sentences of a language owe their truth: what the sentences mean and how the world is. Consider a standard model theory. If two models disagree on the truth value of a sentence the notion of truth in a model must allow for variation in at least one of these parameters, since if there was no such variation allowed the two models would have to be elementarily equivalent, i.e. make the exact same sentences true. So, if the model theory does not allow for variation in how it represents the world to be (i.e., in how the world is), differences in truth value between models must be due to variation in the second parameter, the interpretations of the terms. Hence, if a model 
theory is not involved with representations of how the world is it must be involved with interpretations of what the terms mean insofar as there are differences in truth values between models. Truth in a model will therefore represent the phenomena of truth under an interpretation, and since, as we have already seen, there are no other possibilities involved, it will represent truth under an interpretation in the actual world. The model theory, if it is not representational, must therefore view the models in accordance with (INT) and (MRA) above.

\subsubsection{The Attractiveness of Tarski's Account}

On Tarski's account, an argument is logically valid if and only if no matter what the non-logical parts of the argument are taken to mean, the argument will, as a matter of fact, preserve truth. Etchemendy points out two things about this feature of Tarski's account of logical consequence:

- It makes Tarski's definition extremely attractive.

- It makes Tarski's definition fundamentally mistaken.

The next few sections will engage with spelling out the reasons behind the second claim, as well as discuss some replies that have been offered to the content of Etchemendy's criticism.

However, before turning to why Tarski's account is mistaken, we should briefly comment on why it is attractive, and relate that to the second claim. Tarski emphasizes at the start of his article that the concept of logical consequence is not open for arbitrary definition and that in giving a rigorous definition of the concept we must try to adhere with common, everyday usage of the concept. On the other hand, Tarski also points out that no one definition of logical consequence can possibly respect all everyday intuitions and judgements we may have about this concept. Giving a rigorous definition of logical 
consequence is to a large extent a matter of give and take. Presumably, however, we want our rigorous definition of the concept of logical consequence to respect those intuitions about the concept of logical consequence which we take to be essential to it.

Among some of the characteristics that are often taken to be essential to logical consequence are both Formality and Necessity, as these were presented in a previous section. Other characteristics, often considered essential, are those of being able to know a priori that the relation of logical consequence obtains, ${ }^{40}$, the truth of the conclusion being absolutely certain upon the truth of the premises, and logic being normative: one is somehow making a mistake when endorsing the premises of a valid argument but denying the conclusion. ${ }^{41}$ All of these characteristics, of course, find different exact formulations by different authors, and some are naturally taken to entail, and to be entailed, by these and other characteristics that the consequence relation is taken to possess. The important point to note is that the concept of logical consequence $i s$ seen as essentially linked to concepts such as necessity, a prioricity, and certainty, and it matters less, for our present purpose, exactly how these characteristics are spelled out.

Among the characteristics claimed for logically valid arguments are the following: If an argument is logically valid, then the truth of its conclusion follows necessarily from the truth of the premises. From our knowledge of the premises we can establish, without further investigation, that the conclusion is true as well. The information expressed by the premises justifies the claim made by the conclusion. And so forth. These may be vague and ill-understood features of valid inference, but they are the characteristics that give logic its raison d'être. They are why logicians have studied the consequence relation for over two thousand years.

\footnotetext{
40 To which, some argue, it should be added '... if it can be known at all' (Hanson [1997: 378]).

41 This latter criteria is imposed by Beall \& Restall as a necessary condition on being a logical consequence relation [2006: 16].
} 
In spite of the importance of these characteristics, we needn't be happy about the vagueness. And that is why the reductive analysis of consequence is so attractive. Tarski shows us, if he is right, how the logical properties can be reduced to well-understood notions of truth, plus whatever is involved in specifying the associated generalization or class. Like magic, the vague and obscure notions that sit at the core of our discipline simply disappear. No wonder we find Tarski's account so appealing: if it works, it allows us to set aside a breathtaking number of philosophical issues. Who could not want the account to succeed? (Etchemendy [2008: 265-266])

In the first part of the quote Etchemendy is listing some of the characteristics which we associate with logical consequence. The involved notions, however, are not exactly well understood. There are, of course, proposals as to how to give content to these concepts in the literature, but there is also still a great debate as to what necessity, a prioricity, and certainty consist in, and as of now, no consensus has been reached. However, if Tarski is right in his account of logical consequence, then we need not bother about such issues, as they can simply be sidestepped. All that is involved in Tarski's definition of logical consequence is the definition of truth in a model seen in accordance with (INT) and (MRA), something which is equivalent to the truth of a certain universal generalization. And defining truth in a model, in this manner, can certainly be done without mentioning, or relying on, any of the troublesome concepts, as Tarski's own work has shown (Tarski [1956a, 1956b]). Hence, Tarski's definition of logical consequence and logical truth is extremely attractive, since it allows us to set aside a breathtaking number of philosophical issues, as Etchemendy puts it. We get necessity, a prioricity, centainty, and other features of logical consequence, so to speak, for free. However, Etchemendy's main point is that the attractiveness of Tarski's account is only apparent. There is no reason to think that valid arguments on Tarski's 
account possess any of the essential features of logical consequence. Hence, we do not get these features for free, we do not get them at all. This, of course, is mainly a conceptual point: there is nothing in Tarski's account which guarantees that logically valid arguments possess the features in question.

Many logicians and philosophers react to my conceptual critique of Tarski's account by retreating to an extensional stance, saying the only thing that really matters is that the analysis be extensionally correct. I have no doubt that, when push comes to shove, Tarski would have said the same thing - as, in fact, would I. [2008: 279]

Etchemendy is here pointing out that, as a last resort, the defender of Tarski could justifiably retreat to what Etchemendy calls 'an extensional stance'. The only thing that matters is that the extension of the defined concept coincides with the extension of the genuine relation of logical consequence. Two important notions in relation to the extensional adequacy of the account is that of overgeneration and undergeneration. The former happens when the extension of the defined concept contains an argument not in the extension of the genuine concept, the latter when there is an argument in the extension of the genuine concept which is not in the extension of the defined concept. Let $G C$ the class of genuinely valid arguments in some interpreted formal language $L$. A definition of logical consequence which produces the class $D C$ of valid arguments for $L$ will overgenerate or undergenerate in accordance with the following definitions:

(o-gen) There is an argument $\langle K, X\rangle$ s.t. $\langle K, X\rangle \in D C$ and $\langle K, X\rangle \notin G C$.

(u-gen) There is an argument $\langle K, X\rangle$ s.t. $\langle K, X\rangle \notin D C$ and $\langle K, X\rangle \in G C$.

Etchemendy does not deny that there are some languages for which we can prove that overgeneration does not take place when applying Tarski's definition. But since Tarski's account is conceptually flawed, this cannot be proved from the definition itself. 
I will eventually argue that with suitably weak languages the definition does get the extension right. But even in these cases we must seek external guarantees of that fact. (Etchemendy [1990: 8])

The external guarantee which Etchemendy is referring to is a completeness theorem for a deductive system which can be seen to possess a certain quality. Let $\Gamma \vdash \alpha$ mean that $\alpha$ follows from $\Gamma$ in this deductive system.

(comp) For all $\Gamma$, $\alpha$; if $\Gamma \models \alpha$, then $\Gamma \vdash \alpha$.

If, by inspecting the axioms and rules of inference of the deductive system we can assure ourselves that all deductively valid arguments are genuinely valid, we get the following premise, where $\Gamma \Rightarrow_{G} \alpha$ means that $\langle\Gamma, \alpha\rangle \in G C$ :

(premise1) For all $\Gamma, \alpha$; if $\Gamma \vdash \alpha$, then $\Gamma \Rightarrow_{G} \alpha$.

Together, (comp) and (premise1) entail that all model-theoretically valid arguments are genuinely valid. But, and this is Etchemendy's point, even in cases where this can be shown, the proof is due to a factor that is external to Tarski's model-theoretic definition: the deductive relation being complete with respect to the former and the deductive system being sound with respect to the genuine consequence relation.

Suppose we could also ascertain that our model-theoretic definition of consequence does not undergenerate. That if an argument is not model-theoretically valid, then neither is it genuinely valid. Contraposing:

(premise2) For all $\Gamma$, $\alpha$; if $\Gamma \Rightarrow_{G} \alpha$, then $\Gamma \models \alpha$.

Combining (comp), (premise1), and (premise2) we get that:

(CE) For all $\Gamma, \alpha ; \Gamma \vdash \alpha$ iff $\Gamma \models \alpha$ iff $\Gamma \Rightarrow_{G} \alpha$.

Arguments which aim to show (CE) from the above are known as 'squeezing arguments' ${ }^{42}$ Squeezing arguments are arguments which seek to bridge the gap between

\footnotetext{
${ }^{42}$ Squeezing arguments have their origins in Kreisel [1967]
} 
rigorously defined concepts and informal notions of those very concepts, and they are therefore not confined to considerations about logical consequence.

However, it is important to note that informal does not mean intuitive (Smith [2011: 26ff]). Though our notion of $\Rightarrow_{G}$ is informal it is not intuitive in the sense of having no sharp boundaries. It is an informal, but somewhat precise notion. Of course, this will have to be the case if we are to make judgements about the implications of $\Gamma \Rightarrow_{G} \alpha$ for arbitrary $\Gamma$ and $\alpha$. As Smith [2011: 26ff] points out, squeezing arguments simply cannot be employed until we have an informal notion which is, so to speak, "sharp enough" for us to be able to prove the requisite premises, that of (premise1) and (premise2) above. One might therefore speculate as to whether a defender of Tarski under 'a purely extensional stance' could produce a squeezing argument to show that Tarski's account produces the right extension. We will have something to say about this in chapter 2, when we look at squeezing arguments.

\subsection{Etchemendy's Criticism}

There are two main parts to Etchemendy's criticism of Tarski's account:

(I) Tarski's definition of logical consequence is conceptually flawed.

(II) When applying Tarski's definition to an arbitrary language, there is no assurance that it will yield the right extension.

The content of (I) is quite simple. Tarski's acccount of logical consequence confuses cause and effect. Logically valid arguments preserve truth under all interpretations because they are valid. What Tarski's account does is to turn this around and propose that logically valid arguments are valid because they preserve truth under all interpretations. Because this is the case, we cannot be sure that when applying the definition to an arbitrary language, it will yield the correct extension. If our definition captures 
the wrong extension when applied, then we have obviously not captured the correct concept. So (II) provides support for (I), if accepted.

The content of (I) and (II) can be summed up in the following:

(III) When applying Tarski's definition to an arbitrary language, there is no internal assurance that it will yield the right extension.

If the definition of logical consequence captured the correct concept, then we should be able to ascertain, purely on the basis of the definition, that it will capture the correct extension. I call such an assurance, one that flows directly from the definition, internal as opposed to external. An internal assurance, we may note, ought be the mark of a successful analysis of the target phenomenon. If Tarski's definition of logical consequence did indeed present us with an analysis - that is, told us what logical consequence really $i s$ - then such an assurance should be obtainable from the very definition itself. $^{43}$

${ }^{43}$ If the definition truly captured the concept, the question of "how do we know that $\langle K, X\rangle$ really is valid?" would seem, at least to a certain extent, pointless or confused. Contrast this with the situation mentioned by Etchemendy [1990: 2] of defining the notion of a real closed field; how do we know that a real closed field has the same first-order properties as the field of real numbers? That, as it were, is just the definition of what it is to be a real closed field, and the question therefore seems confused. If someone sincerely asked how we know that a given real closed field has the same first-order properties as the reals, the proper assessment, I take it, would be to conclude that the person in question did not grasp the concept of a real closed field in the first place (provided, that is, that he grasps the other concepts involved in his inquiry). Now, for reasons touched upon in section 1.1 the situations are not completely analogous. The mathematician was free to introduce the notion of a real closed field however he wanted, whereas the situation is not like this for logical consequence. However, as a very minimum, it seems reasonable to suggest that the fact that a definition captures the right extension should at least be recognizable, though perhaps not obviously so, from the definition itself, if the definition is to enjoy the status of an analysis of the concept in question.

Though I did not have it in mind at the time of writing, it has since been pointed out to me by Cei Maslen that the point here is, if not identical to, then at least related to, Moore's Open-Question argument (Moore [1903: $\S 13]$ ). Moore argues that if a certain property X, given in naturalistic terms, is equivalent to the property of being good, then the question "Is it true that X is good?" should be meaningless. However, on account of such questions not being meaningless (but rather being open questions, that is, questions that can meaningfully be asked by a speaker competent with the involved terms), Moore argues that the various naturalistic candidates for $\mathrm{X}$ - candidates for being equivalent to the property of being good - fail. It is not my purpose to assess Moore's argument. I should like to point out that I am not making the strong claim that the question "Is $\langle K, X\rangle$ really valid? should be meaningless when $\langle K, X\rangle$ fits the definition of a valid argument and that definition is given the 
It is generally acknowledged that if a definition captures the wrong extension, then that definition cannot be correct in an intensional sense; that is, the definition cannot count as an analysis of the given phenomenon. Of course, this is true for the case of logical consequence as well. What this means, is that the following claim, related to (II), provides support for both (I) and (II).

(IIa) For a number of languages, applying Tarski's account generates an extension of the concept of consequence at odds with the genuine extension.

If Tarski's account generates the wrong extension, then we cannot have captured the correct concept and there can be no guarantee internal to the definition that it will yield the correct extension.

There are those who would claim that all that really matters is not whether we have the right conceptual analysis - whatever that may mean-but that the extension of the defined concept coincides with the correct concept. We may note in response that the truth of claim (IIa) is evidence against Tarski's definition capturing the correct extension.

We might consider the call for an analysis of logical consequence and the thought that all that matters is the extension of our defined concept as opposite ends of a status of an analysis of the phenomenon of logical consequence. Rather, what I do take myself to be committed to, is that the question "Is $\langle K, X\rangle$ really valid?" should at the very least be able to be answered affirmatively drawing only on the content of the definition itself when this definition is to enjoy the status of an analysis of the phenomenon of logical consequence.

In the same vein I should like also to make clear that I am not claiming that any succesful analysis should necessarily provide us with a method for determining the extension of the defined concept. The point is rather that if we have a succesful analysis of a given concept, then the question of that it has the right extension, rather than the question of whether this or that is in the extension, should be able to be affirmatively answered reflecting only on the content of the analysis. Consider, by analogy, a utilitarian analysis of right action as the action which produces the most utility. By affording this the status of an analysis we do not mean that for each action it should be apparent whether it does in fact produce the most utility. This question will depend on countless empirical features in a way that might make the assessment impossible for practical purposes. But to say that we are dealing with an analysis of the concept of right action, we should at least be able to answer affirmatively, on the assumption that action $A$ does provide the most utility, the question of whether action $A$ is the right action, by reflecting only on the content of the definition which is afforded the status of an analysis. 
spectrum. At one extreme there is the call for a correct conceptual analysis: an analysis that tells us exactly what the concept of logical consequence consists in. This analysis should make it clear how the logically valid arguments come to possess the requisite guarantee of truth-preservation which genuinely logically valid arguments possess. At the other extreme there is only the condition that our definition must have the right extension; the reasons for having that very extension are not relevant. Etchemendy claims that Tarski's account of logical consequence fails for both sets of standards.

We will concern ourselves mostly with the former claim, that Tarski's account fails to provide any kind of analysis of logical consequence. Etchemendy's attack on Tarski's account is in his own words just one single, extended argument (Etchemendy [1990: 8]). Everything that is wrong with Tarski's definition can be traced back to the conceptual flaw in the definition.

The first point we will look at is Etchemendy's attribution of what he calls 'Tarski's Fallacy' to Tarski. This is the fallacy of thinking that it can be proved, on the basis of Tarski's account, that there is a modal connection between premises and conclusion in a valid argument.

This leads us naturally into what I will call 'The Class Membership Argument', which is, in essence, just a reformulation of the source of the fallacy in any attempted proof of the fact that logically valid arguments do possess the requisite guarantee. What the Class Membership Argument shows is that absolutely no guarantee of not having true premises and a false conclusion, whether it be cashed out in epistemic, metaphysical, semantic, or logical terms, can be seen to follow from Tarski's definition. This means that however we do construe the guarantee - a guarantee which we take to be essential to logically valid arguments - it does not follow from Tarski's definition. This, as we have already alluded to, shows two things: Tarski's analysis cannot count as a piece of analysis, and if the arguments valid on Tarski's account do possess the 
requisite guarantee, then they do so by mere coincidence, and evidence of this fact must be external to Tarski's definition.

It is sometimes claimed that Etchemendy's criticism of Tarski cannot be accepted since Etchemendy criticizes Tarski for not capturing the intuitive notion of consequence, but at the same time he does not make it clear exactly what this intuitive notion is supposed to be. The thought seems to be that since Etchemendy does not make it clear what intuitive notion of consequence Tarski's account fails to capture, we are unable to assess these claims.

But Etchemendy's argument is not the following: There is one (and only one) intuitive notion of consequence and Tarski's account does not capture it. Instead, Etchemendy's argument is fully in line with the claim that there is no single intuitive concept of logical consequence, but rather, as Smith [2011: 28] expresses it, an inchoate jumble of ideas. What Etchemendy does point out, is that on each legitimate way of making this inchoate jumble clear, and there are certainly many ways to do so, we end up with a notion which Tarski's account does not capture, simply because each such clarified notion will have as an essential characteristic that of the truth of the premises guaranteeing the truth of the conclusion. There are, of course, many informal, though theoretically robust, notions of logical consequence. All of these can be seen as one way of making the inchoate jumble of ideas precise. But common to all, be it classical, relevant, or intuitionistic conceptions, is the aforementioned guarantee. Tarski's account cannot provide such a guarantee, and as such, Etchemendy's critique of Tarski is not weakened by the observation that it is not clear what exact notion is meant to be captured by Tarski's account; on the contrary, it draws its strength from not having to take a stand on this issue.

The only notion of a guarantee, which can be seen to flow from Tarski's definition, is purely linguistic. This is a guarantee that there can be no semantically well-behaved 
language in which the premises of a valid argument express something that is actually true while the conclusion expresses something which is actually false, provided the logical terms retain their original meaning. But this, obviously, is not the guarantee we want: the guarantee we are after is a guarantee that if things are thus and so, then other things must be a particular way. Consider Prior's ett-operator: $p$ ett $q$ is defined to be true if and only if $p$ and $q$ are both true or Oxford is the capital of Scotland (Prior [1964: 194]). As it just so happens there are plenty of interpretations of $p$ and $q$ making $p$ ett $q$ false just as there are plenty of interpretations of $p$ and $q$ making $p \wedge q$ false. In fact, in the actual world we cannot tell the two apart because their extensions coincide. But once we consider the possible states of the world, how the world is or could be, we see that $p$ ett $q$ is always true in a possible circumstance in which Oxford is the capital of Scotland. This, in essence, is the flaw in Tarski's account: it does not take into account other ways the world could have been. To use the dichotomy from above of language versus the world, the guarantee we are after is a guarantee about the world, about how the world would have to be given that certain other things were the case. Tarski's account cannot guarantee this because it cannot get "outside of the actual world", so to speak. At this point the proponent of the Tarskian account will no doubt urge that ett is not a legitimate logical constant. This, I suppose, should perhaps be granted, though for pragmatic reasons; because its behavior throughout the models as a logical constant is intractable. We will come back to this point about the pragmatic criteria by which we may choose our logical constants. However, the ettoperator nicely illustrates the way in which Tarski's account only depends on how the world actually is. From the perspective of this account ett just is the truth-function $\wedge$. From the perspective of (INT) and (MRA) there is no way of telling ett and $\wedge$ apart. Only insofar as we are willing to speak about how the world could have been can we see that ett is a different truth-function than $\wedge$. But this is exactly what we cannot do 
on an account that accepts (MRA), since only the actual world matters. This way of explaining the illegitimacy of ett is therefore not open to someone who accepts (MRA).

Consider the inference from $\neg p$ to $\neg(p$ ett $q)$. In a model theory meeting the constraints given by (INT) and (MRA) this is guaranteed to preserve truth, since Oxford is not the capital of Scotland, and $\neg(p$ ett $q)$ just is either $\neg p$ or $\neg q$ and Oxford is not the capital of Scotland. Any actual interpretation making the premise true will therefore make the conclusion true as well. But in a possibility in which Oxford $i s$ the capital of Scotland this inference is not truth preserving for any $p$ or $q$.

Also, it is worth noting that the problem is not solved by ruling out the ett-operator as illegitimate. Considering only the standard logical operators, the problem remains. I will illustrate and stress this point by imagining someone who is skeptical about whether the world must be as described by the conclusion of a valid argument if it is as described by the premises, in a language where the choice of logical constants is the standard one. Tarski's definition can offer nothing to lay this skepticism to rest.

The extension of Tarski's account turns on empirical facts about the world, one notable such fact being the size of the universe. But as the preceding paragraph points out, with certain other choices of logical constants, other empirical facts become relevant: for Prior's ett-operator it is relevant whether Oxford is the capital of Scotland. Etchemendy illustrates this point using formal sentences that are true in all models, yet do not intuitively correspond to a logical truth, because they express certain facts about the cardinality of the world, something which logic should intuitively not pass judgment on. It is in a sense unfortunate that Etchemendy has focused on matters of cardinality to such a great extent in presenting his arguments. Of course, this is not to say that these arguments are not good arguments, but rather that they have sparked the subsequent literature into focusing on considerations having to do with the 
cardinality of the world. Such a debate, of course, is not pointless in and of itself, but it does serve to take focus away from the conceptual flaw in Tarski's account, a flaw that remains when the cardinality issues are settled, as these are merely symptoms of the problem, not the cause.

Etchemendy points out that the failure of Tarski's account is sometimes blamed on an incorrect division of terms into logical and non-logical. Tarski's account is not conceptually flawed, but rather, it is simply incomplete. What is needed is a careful analysis of what makes a term logical. When these, and only these, terms are selected as the logical terms, the guarantee will be a consequence of Tarski's definition and the special features of these logical terms (Etchemendy [2008: 269]). Etchemendy, of course, denies this, and argues that there can be no such special features of the logical terms that would be able to secure the aforementioned guarantee.

If we treat only the formal counterparts of certain terms as logical constants, we can give a plausible proof that model-theoretically valid arguments are necessarily truthpreserving, and hence, we can explicate the requisite guarantee in terms of the latter. However, this proof requires us to see the models of the model theory as something which they are not on Tarski's account, namely representations of other possible circumstances. Etchemendy's argument is not meant to deny this. Rather he is arguing that if we accept Tarski's account of what our models represent, then we can prove that there can be no such special features of the logical constants that secures the guarantee in question. However, it is worth noting that Etchemendy denies that there is a principled distinction between logical and non-logical terms. From such a perspective the proof can only show that certain logical constants are a good choice of such, not that they are more logical than other terms. 
As already pointed out, Etchemendy's argument and points are all part of one extended argument. For this reason none of the points to be discussed are completely independent, and I apologize in advance if it seems I am repeating myself.

\subsubsection{What Etchemendy's Criticism is Not}

It may seem strange to have a section on what Etchemendy's criticism is not. But in the present context it is actually crucial to get clear on this. It is important to understand that Etchemendy's criticism is not of model-theoretic accounts of consequence per se, but of of a certain view of such model-theoretic accounts - a view where the models are seen as representing the actual world as well as providing an interpretation to the non-logical constants, that is, a view where the models are seen in accordance with (INT) and (MRA). In Etchemendy [1990] such a model-theoretic account is referred to as interpretational, whereas Etchemendy [2008] refers to Tarski's reductive account of consequence, in the sense of reducing the logical properties to actual truth and generalization. ${ }^{44}$

That Etchemendy is not out to criticize model theory is supported by [2008], where he presents his positive views of model theory, a view that leaves all the technical details of the model theory untouched, but denies the view of models which result from accepting (INT) and (MRA). This is Etchemendy's representational view. Since the two views are different, the criteria used in assessing the success of the model theory change accordingly. Most prominently, Etchemendy does not see himself as giving an analysis of logical consequence, since the notion of a logical possibility is assumed and used in setting up his representational model-theoretic semantics, and the adequacy of the model theory is assessed accordingly. There are some subtleties involved in

\footnotetext{
${ }^{44}$ In fact, in Etchemendy [2008] Tarski's account as it applies to a model theory is not discussed. Instead, the account is presented as given by the relation between an argument and a certain universal generalization. This further strengthens our claim that the target of the critique is not model theory per se, but a certain view of model theory.
} 
Etchemendy's claim that his own representational view does not provide an analysis of the target phenomenon, which will concern us later. It seems that what Etchemendy actually means to say is that this is not a reductive analysis, in the sense of Tarski's definition, but even this fact, it seems, could be remedied by providing a reductive analysis of the notion of a logical possibility.

I am not denying, and neither is Etchemendy ([2008: 263]), that The Concept of Logical Consequence legitimately was the cause of some confusion. One reason seems to be that Etchemendy, in parts of CLC, seem to use the terms 'the model-theoretic account of logical consequence' and 'Tarski's account' synonymously.

Another potential source of confusion, no doubt, is the fact that while Etchemendy makes two claims - that Tarski's account, given in terms of contemporary model theory, is mistaken, and that Tarski's original account is mistaken - the first claim is not explicitly discussed. Of course, if Tarski's account is fundamentally mistaken, then spelling it out in terms of contemporary model theory will not help the situation, so the latter implies the former. The majority of Etchemendy's arguments focus on certain features of Tarski's definition which follow straightforwardly from Tarski's presentation, such as the fact that the $\operatorname{argument}$ from $K$ to $X$ is valid if and only if $\left(^{*}\right)$ is simply true (see section 1.1.5). Of course, Etchemendy's arguments do not become better or worse depending on whether model theory is explicitly considered. However, the situation does make it harder for an individual reader to assess exactly what Etchemendy is saying about model theory. For the purposes of getting clearer on this, I will present Etchemendy's arguments against Tarski's account at a model-theoretic level, though I will engage with Etchemendy's original arguments at points too. ${ }^{45}$

\footnotetext{
${ }^{45}$ Obviously, this strategy will be unjustified to anyone who does not believe that Tarski's original account gives rise to a model-theoretic definition of logical consequence which endorses (INT) and (MRA). However, as I am presenting arguments that Etchemendy did put forward against Tarski's original account, we will at points have to discuss the adequacy of Etchemendy's interpretation of
} 


\subsubsection{Tarski's Fallacy}

Suppose the argument from $K$ to $X$ is valid on Tarski's account and that $K$ consists only of true sentences while $X$ is false. Then, consider the sequence consisting of the objects which are, as a matter of fact, designated by the non-logical terms in $K$ and $X$. This sequence will be a model of $K$ but not a model of $X$. Contradiction.

This proof is remarkably simple. As Etchemendy uses the term Tarski's Fallacy it applies to thinking that the above proof shows that if an argument is valid on Tarski's account, then it is impossible for the premises to be true while the conclusion is false. What the above proof shows is that it is impossible for an argument to be valid on Tarski's account and have true premises and a false conclusion. But when we reflect on Tarski's account of logical consequence this should hardly be surprising. Of course no argument will be valid on Tarski's account and not be truth-preserving, since being truth-preserving is a condition on the argument being valid in the first place.

The above "proof" does not rely in any way on which terms of the language we are designating as logical.

- Copenhagen is the capital of Denmark.

- Therefore, Wellington is the capital of New Zealand.

Suppose we designate 'Copenhagen', 'Wellington', 'being the capital of Denmark', and 'being the capital of New Zealand' all as logical terms. Then it should be clear that the argument is valid on Tarski's account, yet it should be just as clear that there is absolutely no modal connection between the premise and conclusion. In fact, there seems to be no connection at all between the two. But since the alleged proof does not mention the selection of logical terms, the result should hold independently and so hold for this argument as well. Thus, thinking that the proof shows any modal connection Tarski's account as giving rise to a model-theoretic account endorsing (INT) and (MRA). 
is clearly a fallacy. Defenders of the Tarskian tradition will often reply that this proof is flawed exactly because it does not take into account the properties of the correct logical terms of the language (Sher [1996: 654ff]). Such a reply is not, as is perhaps thought, a counter to or criticism of Etchemendy's observations. Independently of the choice of logical terms, any proof that shows that a modal connection exists between premises and conclusion on a Tarskian model-theoretic account will undeniably have to see the models as representations of possible circumstances.

At this point we may observe that the fallacy remains when we turn to modeltheoretic characterizations that endorse (INT) and (MRA). Assuming that the argument $\langle\Gamma, \alpha\rangle$ is truth-preserving in all models but that $\Gamma$ consists only of true sentences while $\alpha$ is false, yields an immediate contradiction. Consider the model representing the interpretation which the non-logical constants of $\Gamma$ and $\alpha$ actually have. The argument will not be truth-preserving in this model. Contradiction.

As Etchemendy [1990: 87] points out, such a proof shows only the following:

A) $\square(p \rightarrow(q \rightarrow r))$

Here $\square$ is meant to be an intuitive necessity operator, $p$ represents a given argument being model-theoretically valid, $q$ represents that the given argument has true premises and $r$ represents that the argument has a true conclusion. What we can prove is that it is necessary that if an argument is valid, then if the premises are all true, then the conclusion is true too. However, to get a modal connection between premises and conclusions, we would need to show that if an argument is valid, then it is necessary that if the premises are true, so is the conclusion. That is, the following:

B) $p \rightarrow \square(q \rightarrow r)$

But inferring $B$ from $A$ involves a modal fallacy, hence, the name Tarski's Fallacy. Of 
course, pointing out that $B$ must be false because it does not follow from $A$ would be equally fallacious, and that is not Etchemendy's point. Etchemendy's point, rather, is that there is nothing in the Tarskian model-theoretic account which excludes $B$ from being false.

Let us investigate $B$ in some more detail. $p$ represents a given argument being model-theoretically valid on an account that endorses (INT) and (MRA). Each model represents an interpretation of the non-logical constants in the actual world, and together the models represent all such interpretations. What this means then, is that the argument in question is truth-preserving, in the actual world, no matter how we interpret the non-logical constants. Hence, the logical validity of an argument is equated with a certain class of arguments all being truth-preserving. This class contains, besides the argument itself, the arguments that can be obtained from the argument by providing the non-logical terms with a new interpretation. ${ }^{46}$

The crucial point is this. The property of being logically valid cannot simply consist in membership in a class of truth-preserving arguments, however that class may be specified. For if membership in such a class were all there were to logical consequence, valid arguments would have none of the characteristics described above. They would, for example, be epistemically impotent when it comes to justifying a conclusion. Any uncertainty about the conclusion of an argument whose premises we know to be true would translate into uncertainty about whether the argument is valid. All we could ever conclude upon encountering an argument with true premises

\footnotetext{
${ }^{46}$ We may note that there is no guarantee that all of these arguments can be given in the language in question. This will be the case only if the language contains a means of referring to all objects of appropriate type. However, we can think of each argument in this associated class as being given in a different language, where the meanings of the non-logical terms differ in order to make them designate all objects of the appropriate kind.
} 
would be that either the conclusion is true or the argument is invalid. For if its conclusion turned out to be false, the associated class would have a non-truth-preserving instance, and so the argument would not be logically valid. Logical validity cannot guarantee the truth of a conclusion if validity itself depends on that same-self truth. (Etchemendy [2008: 267]).

The characteristics which Etchemendy is here referring to are the characteristics standardly associated with logically valid arguments. If being logically valid simply consists in being a member of this class, then logically valid arguments would possess none of these characteristics. Etchemendy is not denying that some arguments, which are members of this class, do possess these characteristics: after all, modus ponens arguments are valid on this account, and surely such arguments do possess the mentioned characteristics. The problem is not that none of the valid arguments possess any of these characteristics, but rather that the definition does not guarantee that they do. The claim is that accepting the model-theoretic account of logical consequence, which results from accepting (INT) and (MRA), cannot guarantee the truth of $B$ and other related claims expressing the possession of other features we might take to be essential to logical consequence. $B$ might be true, but the reasons for $B$ being true will be unrelated to the given account of logical consequence. It is for this reason that the Tarskian model-theoretic account does not provide us with an analysis.

Of course, if $B$ followed from $A$, we would have such a guarantee, but this is exactly what is not the case. Instead, $A$ is all we can be sure holds of the relationship between logically valid arguments and their premises and conclusions. And this is exactly what makes it the case that none of the characteristics are guaranteed to apply. An equivalent way of putting $A$ is as follows:

$A *) \square(\neg p \vee \neg q \vee r)$ 
$\neg p$ represents that an argument is not valid on the Tarskian model-theoretic account, which, as we have just seen, is equivalent to the associated class of arguments having at least one non-truth-preserving argument. $A *$ now tells us that we are guaranteed that for each argument we can muster: there is at least one non-truth-preserving argument in the associated class of arguments or the argument has at least one false premise or the conclusion is true. This, Etchemendy claims, is not sufficient. Upon encountering an argument with true premises we are guaranteed the following: either the argument has a true conclusion or the argument is invalid.

\subsubsection{Definitional vs. Epistemic Order}

Etchemendy quite explicitly says that "validity cannot guarantee the truth of a conclusion if validity itself depends on that same-self truth" [2008: 267]. Priest [1995] identifies Etchemendy's claims to be the following:

If the validity of an inference is to be identified with the truth of a universal generalization, then we cannot know that an inference is valid unless we know this generalization to be true. But we cannot know that this generalization is true unless we know that its instances are true; and we cannot know this unless we know that every instance of an argument form is materially truth preserving. Hence, we could never use the fact that an argument form is valid to demonstrate that an argument is truth preserving. Thus the prime function of having a valid argument would be undercut. [1995: 287]

Since the logical validity of an argument depends on the truth-preservation of all arguments in a certain class, given that the original argument is in this class, we cannot know that all arguments of this class are truth-preserving without knowing that the 
original argument is. Or, in other words, we cannot know that an argument is valid without first knowing that it preserves truth. This undercuts the prime function of a valid argument, that of drawing new conclusions upon premises we know to be true. However, as Priest goes on to argue, the undercutting is only apparent.

This argument, in fact, fails. This is simply because epistemic order need not coincide with definitional order. To see this, just return to the definition of effectivity provided by Church and Turing, and note that we can be sure that a process is algorithmic before we check to see whether we can produce a Turing machine to perform it. If we could not, the Church/Turing thesis would have no bite. Similarly, even if the definition of validity is correct, we may be able to determine that an inference is valid without consulting the definition. This, of course, raises the question of how we tell that an inference is valid (or that a procedure is algorithmic). But that is another issue. [1995: 288]

What Priest is pointing out is that the problem that pertains to valid arguments on this account, i.e., that they cannot guarantee the truth of their conclusions since their very validity depends on this truth, is solved by pointing out that definitional order need not coincide with epistemic order. Definitional order, as used here by Priest, indicates a certain relationship between a definition and a thing fitting that definition, whereas epistemic order indicates how we come to know that a certain thing fits the definition. If the question of whether an argument fits the definition (epistemic order) can be settled without reference to the definition itself (definitional order), then the problem disappears. This, Priest admits, will of course leave us with the question of how we come to know that an argument is valid, but that is an entirely different question.

Etchemendy accuses Priest of having misunderstood his argument. 
Priest has misunderstood my argument, the point of which is that it is possible for an argument that is not in fact logically valid - one that has none of the epistemic or other characteristics of a valid argument - to satisfy Tarski's definition. The point has nothing to do with knowing whether Tarski's definition applies, but rather with the characteristics (or lack of characteristics) of the arguments to which the definition could in fact apply. (Etchemendy [2008: 269]).

Etchemendy is denying that his point has anything to do with knowing whether Tarski's definition applies or not. Rather, the point is that an argument could be valid on Tarski's account and not possess the characteristics which genuinely valid arguments possess, a guarantee that if the premises are true, then so is the conclusion. It is this guarantee, however we make sense of it, which does not flow from Tarski's definition. But if this is the case, then genuinely valid arguments and valid arguments on Tarski's account come apart, at least conceptually. This is made clear by pointing out that it is quite possible for an argument to fit Tarski's definition, yet not be valid.

Suppose we have an argument form all of whose instances preserve truth, just as [Tarski's account] requires, but suppose that the only way to recognize this is, so to speak, serially - by individually ascertaining the truth values of the premises and conclusions of its instances. In other words, suppose there is no independently recognizable guarantee of truth preservation as there is with modus ponens, only the brute fact that the instances preserve truth. Would an instance of this argument form be logically valid? Clearly not. For example, we could never come to know the conclusion of such an argument in virtue of our knowledge of its premises. [2008: 268]. 
Etchemendy is here pointing to the possibility of an argument that just happens to have only truth-preserving arguments in its associated class, but where the only way to know this is to inspect all arguments in the class one by one. Such an argument would fit Tarski's definition, but not be genuinely logically valid, and so the two do come apart.

There is a tension present in Etchemendy's work resulting from his way of replying to Priest and a reply to a comment made by Smiley [1998]. Smiley is commenting on the fact that it cannot be proved, on the basis of Tarski's definition, that logically valid arguments preserve truth necessarily. According to Smiley it is not obvious, at least from the outset, that logically valid arguments ought to preserve truth necessarily.

A debate is called for, but it will be more fruitful if it asks for what purpose necessity is an essential feature of logical consequence. For example, someone who does not endorse Aristotle's doctrine of proof and episteme may well be content with proofs that establish the bare truth of theorems, and it is not obvious that this requires a modal relation of consequence. (Smiley [1998: 602]).

Etchemendy's reply is quite clear:

Fine, jettison all talk of modalities. Concentrate on nothing more than the fact that the consequence relation allows us to establish the truth of sentences based on the truth of others. But that is precisely the problem, precisely the characteristic that Tarski's definition ignores. If the consequence relation involved nothing more than what the reductive definition maintains, then the relation could never be used to "establish the bare truth of theorems." When we encounter a new inference of any specified form, our sole guarantee would be that it either preserves truth or constitutes a 
counterexample in virtue of which the argument is invalid. This guarantee, the only one Tarski's definition offers, can never establish the truth of anything. (Etchemendy [2008: 271]).

Etchemendy is once again pointing out that a valid argument on Tarski's account can never guarantee the truth of its conclusion in the requisite sense. But this observation is true only modulo conflating epistemic and definitional order, as Priest warns us against in the above quote. Etchemendy is claiming that even if we rest content that no modal connection needs to be present between premises and conclusion in a valid argument, we still would not get the required guarantee. But here Priest's point does apply: if it is always possible to come to know that an argument is valid independently of ascertaining the truth-preservation of all arguments in the given associated class, then we will have a guarantee that if the premises are actually true, then the conclusion is also actually true too.

Is it plausible to claim that epistemic and definitional order do not coincide? I want to suggest that, at least for the purposes at hand, it is not. Recall that Etchemendy argues that Tarski's account of consequence does not provide us with an analysis of the phenomenon; it does not tell us what logical consequence consists in. One might plausibly claim that such an analysis cannot plausibly be separated from the epistemic question of how we come to know that a given entity falls within the extension.

$(\mathrm{CON})$ If $\Delta$ is an analysis of the phenomenon $\Omega$, in the sense of being the true theory of what $\Omega$ consists in, then a general theory of how we come to know that something falls within the extension of $\Omega$ will involve $\Delta$ in an essential way.

The intuition here is that a theory which allows us to determine, on a case by case basis, whether $x$ is $\Omega$ must somehow be involved with the theory $\Delta$ of what it is to belong to $\Omega$. If not, it seems entirely possible that $\Omega$ lets us pick out the wrong 
thing, and so is not such a theory after all. ${ }^{47}$ While the exact content and motivation for $(\mathrm{CON})$ is certainly interesting, I will not pursue it here. I shall later be arguing that necessary truth-preservation is an essential feature of logical consequence, as we understand the latter. If this is right, then Priest's objection does not apply, since it relies on pointing out that valid arguments can, under certain assumptions, guarantee actual truth-preservation.

One might wonder whether what Priest is in effect doing with his comments is just "retreating to the extensional stance," as we looked at it above, that is, pointing out that all that matters is that the definition captures the right extension. In relation to the current issue, this would be pointing out that whereas the guarantee of truthpreservation does not follow from the definition itself, the valid arguments nevertheless possess it and this is all that matters. While Priest's comments are related to the matter of extensional adequacy, his point is not merely that it should not matter if the features in question follow from the definition or not. Rather, what seems to be the case on the Tarskian account is that the definition itself is incompatible with a certain feature following from the definition. In fact, it follows from the definition that logically valid arguments cannot be used to draw true conclusions upon true premises, since there will always be the two possibilities open that either the conclusion is true or the argument is invalid, and Tarski's account gives us no reason to think that it is one rather than the other. Priest blocks this inference by pointing out that definitional and epistemic order need not coincide.

\footnotetext{
47 The idea is something like this: Suppose $\Delta$ is the true theory of what it is to be $\Omega$. Then, for a possible world $w$, applying the theory of $\Delta$ picks out the extension of $\Omega$ in $w$, call it $\Omega_{w}$. Now, suppose we have a general theory $M$ that lets us decide, truly, for given $x$ and $w$, whether $x \in \Omega_{w}$. The point now is just that if $\Delta$ and $M$ are entirely independent of one another, then it seems there will be $x^{\prime}$ and $w^{\prime}$ such that $x^{\prime} \in \Omega_{w^{\prime}}$, whereas $M$ tells us that $x^{\prime} \notin \Omega_{w^{\prime}}$. Or, in other words, since the former is decided by $\Delta$ and the latter by $M$, if the two give the same verdict for all possible worlds, then there must be a connection between $\Delta$ and $M$ in all possible worlds. But that is just to say that there is a necessary, or essential, connection between the two.
} 
If we accept (CON) (or something very much like it) then accepting Priest's comments will entail retreating to the extensional stance. If Priest is right in his division of definitional and epistemic order when it comes to the Tarskian definition of logical consequence, it follows, if $(\mathrm{CON})$ is accepted, that this definition cannot count as an analysis of logical consequence, as telling us what logical consequence consists in. What we have is instead a definition that happens to deliver an extension which coincides with the genuine phenomenon.

\subsubsection{The Reduction Principle}

'The reduction principle' is the name given by Etchemendy to describe the principle which reduces the validity of the argument $\langle K, X\rangle$ to the truth of $\left(^{*}\right)$, which was given as the claim $\forall x_{1}^{1}, \ldots, x_{k}^{1}, \ldots, x_{1}^{n}, \ldots, x_{m}^{n}\left(\left(\bigwedge_{k^{\prime} \in K^{\prime}} k^{\prime}\right) \rightarrow X^{\prime}\right)$. The term 'reduction',

no doubt, is meant to confer that if this principle were true, we would have a genuine reduction of the logical properties to more manageable ones: that of truth and satisfaction. Etchemendy discusses this principle in relation to the logical truth of a single sentence. A sentence, $X$, is logically true if and only if the following claim is true:

$$
\forall x_{1}^{1}, \ldots, x_{k}^{1}, \ldots, x_{1}^{n}, \ldots, x_{m}^{n} X^{\prime}
$$

Here the variables, $x_{1}^{1}, \ldots, x_{k}^{1}, \ldots, x_{1}^{n}, \ldots, x_{m}^{n}$, are the ones appearing in the sentential function $X^{\prime}$ obtainable from $X$ by Tarski's procedure. In terms of contemporary model theory, as we have already seen, the truth of $X^{\prime}$ for all values of the variables is equivalent to the truth of the $X$ in all models, when the non-logical terminology of $X$ is taken to be precisely the terms of $X$ which are replaced by variables, and assuming that the models are restricted by (INT) and (MRA).

This, Etchemendy points out, is just the following principle:

(RP) If a certain universal sentence is true, then all of its instances are logically true. 
This may at first strike us as reasonable, but if so, Etchemendy urges, it is because we are conflating it with two other principles, which are obviously true. One such is the principle stating that if a universal sentence is logically true, then all of its instances are logically true. This, after all, is just to say that logical truth is itself closed under logical consequence, since any instance of a universal sentence follows logically from the universal sentence.

(OTP1) If a universal sentence is logically true, then all of its instances are logically true. In addition to this, we have another obviously true principle about the ordinary truth of instances of universal sentences.

(OTP2) If a universal sentence is true, then all of its instances are true.

The reduction principle is neither of these two principles and must not be conflated with them. The reduction principle is, so to speak, half (OTP1) and half (OTP2) in that it has the antecedent of (OTP2) and the consequent of (OTP1).

Etchemendy says the following about the implausibility of the reduction principle.

I will not spend much time discussing the abstract acceptability of this principle. Unadorned and unmodified, its implausibility could hardly be more apparent. Our natural inclination is to reject the principle out of hand, to reject it for a very simple reason: universal generalizations have no particular claim to logical truth; they, like any sentences, can be true by mere happenstance. ...

The problem with the reduction principle is that the mere truth of a universal generalization can, in general, guarantee nothing more than the truth of its instances. It cannot guarantee that its instances have any other distinguishing characteristics. (Etchemendy [1990: 99-100]) 
What Etchemendy is pointing out here is that universal truth is different from logical truth, a point which has also been made by Kneale [1961: 95ff] against the plausibility of Tarski's account. A universal truth, of course, guarantees the truth of its instances, but this does not mean that its instances have any special properties, like that of being logically true. There certainly are some logical truths that are instances of universal truths, but in these cases the universal truth will itself be a logical truth. Truth certainly can come about through contingent matters, things that just so happens to be, and this does not change significantly when we consider universally quantified sentence. A universal truth may record only that it just so happens that all of the instances are true. That is, the truth of universal claim can be just as dependent on contingent facts as other sentences.

We will look at Etchemendy's purported counterexamples to the reduction principle in the coming section. Before doing so, we should note that there are at least two different ways to view the reduction principle. We can understand it either as an actual de facto truth or as explicating something about the involved concepts, giving us, as it were, an analysis of the concept of logical truth. To uphold the latter we might claim that a stronger relationship needs to hold between the antecedent and consequent of (RP) than the fact that there merely happens to be no counterexamples. If (RP) is to give us an analysis of the concept of logical truth, counterexamples to (RP) should not only not exist, they should not be possible.

On the other hand, we may treat (RP) as itself simply a truth. Something that is the case.

(RPA) It is actually true that if a certain universal sentence is true, then all of its instances are logically true. 
This is just the claim that, as a matter of fact, there is such a relationship between the concepts of the antecedent and those of the consequent. This would be equivalent to holding that Tarski's definition actually captures the correct extension, and for those who, so to speak, "retreat to the extensional stance," the truth of (RPA) is all that is needed in order to vindicate Tarski's account.

Etchemendy claims that, without serious modifications to Tarski's original account, this claim is false for the model-theoretic account that results, and he presents some counterexamples. The modifications in question have to do with the cardinality of the domains, and whether or not Tarski intended these to vary. Allowing varying domains, though perhaps unmotivated from Tarski's perspective, do not seem unmotivated from a perspective seeking to explicate logical truth as truth under all interpretations, something we will look at below. The upshot is that if we allow varying domains along with the intuitive principle that there are infinitely many objects, the purported counterexamples to (RPA) are no longer available. From the extensional standpoint, therefore, Tarski's account is not threatened by Etchemendy's examples.

From the extensional standpoint, all that matters is that the reduction principle states a truth. However, as we said, if we are to maintain that Tarski's account provides an analysis of logical truth, the reduction principle should reflect something more than just a contingent truth.

$(\mathrm{RPN})$ It is necessarily true that if a certain universal sentence is true, then all of its instances are logically true.

If there are counterexamples to (RPA), then these are automatically counterexamples to (RPN). However, we can explain the purported counterexamples to (RPA) away by pointing out that there are infinitely many objects, and in a similar manner the purported counterexamples to $(\mathrm{RPN})$ can be explained away by pointing out that not only are there infinitely many objects, there must be infinitely many objects. 
Many responses and objections to Etchemendy's work have focused on arguing these two points: that there are infinitely many objects and that this fact is necessary. While such issues are certainly interesting, the fact remains that such considerations show only that Etchemendy's purported counterexamples are not in fact counterexamples. And this should not be allowed to take focus away from Etchemendy's main point: that Tarski's account is conceptually flawed. Removing the counterexamples does not by itself remove the flaw. Hence, considerations of cardinality, insofar as they serve to show the truth of these claims from a Tarskian perspective, serve only to distract from the real problem. The real problem, as I will make clear below, is that logical necessity, understood as the 'must' with which the premises of a valid argument necessitates the conclusion, remains undefined over non-actuality, and this simply does not square with our common understanding of logically valid arguments, as well as the use to which we put such arguments. ${ }^{48}$ We will discuss the purported counterexamples to (RPA) and $(\mathrm{RPN})$ at the model-theoretic level. We thus equate being true in all models with the given universal sentence being true, something which we have seen to be justified when the models are seen in accordance with (INT) and (MRA).

\subsubsection{Substantive Generalizations}

In accordance with (MRA) each model represents simply the actual world, and each model interprets the non-logical terminology in accordance with (INT). Suppose there are $\tau$ existing objects, where $\tau$ is some cardinal number. Then in accordance with what the models represent we have that for each model $M$ and associated domain $D_{M}$, $\left|D_{M}\right|=\tau$, since otherwise both (MRA) and (INT) cannot be upheld as each model represents the actual world and each possible denotation given to a non-logical term is an actual object.

\footnotetext{
48 This point is also made by Shalkowski [2004: $66 \mathrm{ff}]$.
} 
This brought out by considering the sequence of sentences given as:

$$
\begin{aligned}
& \rho_{1} \exists x(x=x) \\
& \rho_{2} \exists x \exists y(x \neq y) \\
& \rho_{3} \exists x \exists y \exists z(x \neq y \wedge x \neq z \wedge y \neq z) \\
& \rho_{4} \exists x \exists y \exists z \exists u(x \neq y \wedge x \neq z \wedge x \neq u \wedge y \neq z \wedge y \neq u \wedge z \neq u) \\
& \text {... and so on. }
\end{aligned}
$$

Now, each sentence contains only logical terms and consequently has only one interpretation: $\rho_{n}$, for a given $n$, expresses that there are at least $n$ objects. But since each model represents the actual world with an interpretation of the non-logical terms, it follows that since there is only one interpretation to respect, each model must have a domain of exactly $\tau$ in order to reflect that the requisite sentences are in fact true under their given interpretation. This, Etchemendy urges, is counterintuitive, since it means that $\rho_{2}$, for example (assuming that $\tau>2$ ), will be a logical truth. But none of $\rho_{n}$ should be a logical truth, for any $n$, since such a sentence expresses something which is certainly a contingent matter. Even though there are $\tau$ objects, there could certainly have been a different number. At the same time, since each of these sentences contains no non-logical terminology, they are all their own universal closure with respect to these non-logical terms. The appropriate universal generalizations - that is, the universal generalizations we obtain by replacing the non-logical terms with a variable and taking the universal closure - are therefore true, but since the sentences themselves do not express logical truths, it seems we have a counterexample to (RPA). ${ }^{49}$

\footnotetext{
${ }^{49}$ We assume that the existential quantifier and identity are both logical constants. Etchemendy does discuss how different choices of logical terms could affect the reduction principle. We will not discuss these matters here. See Etchemendy [1990: 100ff].
} 
At this point the proponent of the account under consideration has a natural reply. There are certainly actual contexts in which 'there is' and phrases like that do not range over all the objects that actually exists. This, of course, is relevant since it shows that there are legitimate interpretations of such phrases as 'there is' and 'everything is such that' in which the quantificational aspect does not range over everything that exists. For example, I may, upon opening my fridge, utter 'There is no more milk'. Clearly, I do not intend to be conveying that there is absolutely no more milk in the actual world, and I would have been speaking falsely had I intended that. The meaning of 'there is' in this context is relative to my fridge - a fridge which contains no more milk. Given that there are legitimate interpretations of the quantifiers in which they range only over subsets of all there is, there should be models containing fewer than $\tau$ objects.

Etchemendy [1990: 69ff] argues that this move is at best unmotivated from Tarski's perspective and at worst inconsistent with it. I will not rehearse these arguments here and refer the reader to Etchemendy's original. From a perspective which endorses that models provide reinterpretations of the non-logical terminology in the actual world- that is, a model theory endorsing (INT) and (MRA) - this move seems not only legitimate, but also forced upon us if we want to capture all possible interpretations. ${ }^{50}$

Accepting this, then, we see that the correct restrictions to put on $D_{M}$ for a given model $M$ is that the cardinality of the former does not exceed that of $\tau$. But, then, since we are concerned with all models meeting the restrictions we can see that $\rho_{n}$ is no longer a logical truth, i.e., true in all models, except for $n=1$.

However, as Etchemendy points out, the opposite problem occurs if we assume that the universe is finite. By the opposite problem we mean that the negations of some

\footnotetext{
${ }^{50}$ Etchemendy [1990: 69ff] suggests something like this treatment for the quantifiers, but argues that it is problematic at best, inconsistent at worst. The idea is that each quantifier has a logical part, that remains stable, and a non-logical part, which decides the nature of the objects quantified over (i.e., the domain). For example, in the above example, the non-logical part of 'there is' is understood to be objects in my fridge. Consequently, 'There is no more milk' means that for every object in my fridge, it is not milk. This, therefore, is true if my fridge truly contains no milk.
} 
sentences $\rho_{n}$, for appropriate values of $n$, become logical truths. Let $k$ be a certain finite number; then $\neg \rho_{k}$ expresses that there are no more than $k$ objects. Now, suppose $\tau$ is in fact one of the natural numbers. Then, since every model $M$ will be restricted by $\left|D_{M}\right| \leq \tau$, it follows that $\neg \rho_{n}$ will be true in all models provided that $n>\tau$. But, once again, $\neg \rho_{n}$ makes a claim about a contingent matter - the cardinality of the universe - and so should not be a logical truth. Since each sentence of the form $\neg \rho_{n}$ contains no non-logical terms, it is its own universal closure with respect to these, and consequently under the assumption that the universe is finite we have a counterexample to (RPA) above.

It may be objected, of course, that the last example is not a counterexample to (RPA), since it relies on the false claim that the universe is finite. The universe certainly is not finite, as witnessed by the myriad of mathematical entities that, though they may be abstract, nevertheless exist. Even if we accept this claim, we could still hold that the universe could have been finite, and in this way we obtain a counterexample to $(\mathrm{RPN})$. If the universe had been finite, $\neg \rho_{n}$ would still be true in all models for $n$ which exceed this finite limit. But $\neg \rho_{n}$ makes a contingent claim about the size of the universe, and so should not be a logical truth.

One way of replying to this criticism is to follow McGee [1992] in claiming that not only are there infinitely many objects, there could not have been only finitely many. This gets rid of the counterexamples to (RPN) given by the sentences $\neg \rho_{n}$, for given $n$. If there are no possibilities in which there are only finitely many objects there are no possibilities where the domains of all models are restricted to be finite.

\subsubsection{The Contingency Problem}

The problem which we have been discussing in the previous section is broader than just considering cardinalities. McGee [1992] dubs it 'The Contingency Problem'. 
$[\mathrm{T}]$ he contingency problem is this: What models there are is a matter of contingent fact. If my counterexample to the thesis that $\varnothing$ is valid is a model whose universe consists of the tigers in the Bronx Zoo, then, if the world had evolved differently so that there had been no tigers, the model would not have existed. But what sentences are valid is not a matter of contingent fact. For a sentence to be valid, it is not enough that there is, in fact, no model in which the sentence is false. We need to know that there couldn't be a model in which the sentence is false. (McGee [1992: 274-275])

One intuitive way to bring out how contingent facts can affect the availability of models is to consider the cardinality of the universe. It is contingent how many things there are, but once it is settled, it puts straightforward constraints on what models there are.

McGee argues that the purported counterexamples to (RPA) and (RPN) having to do with cardinality fail, but in fact he wants to argue further that:

If there might be a model in which $\varnothing$ is false, then there actually exists a model in which $\varnothing$ is false. [1992: 276]

To show this McGee makes use of a principle saying that for any world, $w$, and any model, $\mathbf{M}$, in $w$, there is an isomorphic model which consists of pure sets. He then argues as follows:

Suppose there is a possible world $w$ in which there is a model $\mathbf{M}$ in which $\varnothing$ is false. Since the [principle] is true in every world, there exists in $w$ a model $\mathbf{L}$, isomorphic to $\mathbf{M}$, which is a pure set. Being an object of pure mathematics, L exists in every world, and it is, in every world, a model in which $\varnothing$ is false. In particular, $\mathbf{L}$ bears witness to the fact that, in the actual world, there is a model in which $\varnothing$ is false. [1992: 276] 
From our perspective there are several worrying aspects about this purported proof. Most prominently, of course, it is unclear whether the proof really relies on models being seen in accordance with (INT) and (MRA). This, of course, is no criticism of McGee who is not necessarily trying to prove the result from a perspective of viewing models as such. Secondly, McGee focuses on the fact that there is a possible countermodel, but we need to establish further that if a sentence is false in a possible world, then that world contains a countermodel - at least in so far as we are trying to establish that logical truths are necessary. We will look at these exact issues in the following chapter. We may note that McGee is relying on the principle that if two models are isomorphic, then they will satisfy the same sentences, i.e., be elementarily equivalent. This, of course, is true for our standard model theories and their selection of logical terms. However, it can fail to be true. Consider adding the following predicate, $B$, to a standard first-order language interpreted via a standard model theory, and giving $B t$, for a given term $t$ and model $M$, the following truth conditions:

(tB) $M \Vdash_{v} B t$ if and only if $\operatorname{den}(t)_{M, v}$ is blue.

Imagine a model, $M$, making $B a$ true for some individual constant $a$. In this model then, it follows that the denotation of $a, I(a)$, is blue. The problem with this model is that any isomorphic model consisting of pure sets will make $B a$ false, not trueassuming, of course, that pure sets have no color. What happens here is that the definition of $B$ as a logical constant prevents the model theory from exhibiting the isomorphism property, where the latter means, roughly, that any two isomorphic models will be elementarily equivalent. ${ }^{51}$ A model $M$ might make $B a$ true and be isomorphic to $N$ without $N$ making $B a$ true. McGee is therefore tacitly assuming that certain parts of the language, including $B$, are excluded as counting as the logical constants

\footnotetext{
${ }^{51}$ Shapiro [1998: 152] suggests that any model theory worthy of its name will have to satisfy the isomorphism property. See chapter 2.
} 
of the language. We will have much more to say on this in chapter 2 .

Independently of the above, MacFarlane [2000: 4] points out that Etchemendy's point is not that there could have been no sets, as McGee at one point seems to suggest:

This conclusion of Etchemendy's is doubtlessly correct. A model is a kind of set, and pure logic doesn't require that there exist any sets at all, so that it is logically possible that every sentence should be true in every model. But it is not logically possible for every sentence to be true, and, a fortiori, it is not logically possible for every sentence to be valid. (McGee [1992: 275])

It looks as though McGee is pointing out that Tarski's analysis presupposes the existence of sets, in the sense that it will get the wrong verdict if it turns out that there are no sets. If there are no sets, there are no models, and every sentence will vacuously be true in every model. And while sets may exist with metaphysical necessity, it is not logically necessary that they do. Pure logic, as he puts it, does not require that sets exist.

But this is not Etchemendy's point. To see this it becomes crucial to recognize what the models of the underlying model theory represent. It is what truth in all models represents, on the Tarskian approach, which makes it so that true in all models cannot mean, or be equivalent to, logically true.

Properly understood, [Etchemendy's criticism] is directed against the underlying analysis of logical truth as truth on all possible semantic interpretations of the language's nonlogical vocabulary, not against any particular mathematical realization of that analysis. (MacFarlane [2000: 1]) 
MacFarlane is here pointing out that Etchemendy's criticism is not against model theory but against the particular notion of a logically valid argument which arises from seeing the models in accordance with (INT) and (MRA). The problem is that the model theory models truth under all semantic interpretations of the non-logical terms, and while the model theory is perfectly apt for this purpose the problem is thinking that this latter notion - truth under all semantic interpretations of the non-logical terms - is conceptually equivalent to, or gives any kind of analysis of, logical truth. Had there been another way to model the phenomenon of truth under all semantic interpretations, the same criticism would apply here. Once we realize this we see our way of understanding McGee's argument cannot be entirely correct if it is meant to be a restatement of Etchemendy's point, since it turns on a particular mathematical way of explicating truth preservation under all semantic interpretations, namely standard model theory.

MacFarlane goes on to note that one of Etchemendy's prime examples of a philosophically interesting definition, that of the natural numbers, $\mathbb{N}$, relies on the existence of sets, since the natural numbers can be defined as being the smallest set containing 0 closed under the successor operation. About this definition Etchemendy says:

Now it is perfectly clear that this definition is not identical to the intuitive notion it supplants. Thus, it employs a variety of set-theoretic concepts that are not, by any stretch of the imagination, part of our ordinary understanding of the natural numbers. ... But the definition obviously captures the essential feature of the intuitive notion, and so its extensional adequacy is apparent from the definition itself. We do not, so to speak, have to try it out to see that it really works. (Etchemendy [1990: 9])

Though the concept of a set is not part of our intuitive understanding of the natural numbers, the definition relying on sets is nevertheless adequate in that it captures the 
concept of the natural numbers straightforwardly. If the fault in Tarski's account is that it presupposes the existence of sets, this definition of the natural numbers would be equally faulty. But the point is that this definition of the natural numbers, though certainly not the only possible candidate, can be seen as an analysis: as capturing the essential features of the natural numbers, a fact which is apparent from the definition itself. Consequently, there can be succesful analyses that rely on the notion and existence of sets, and the fault in Tarski's definition is not that it utilizes and relies on the notion of sets, but rather what these sets represent.

As MacFarlane points out, Etchemendy's criticism is that logical truth cannot be equated with truth under all I-PSIs, where the latter is given as:

A interpretational possible semantic interpretation (I-PSI) assigns to each non-logical term $X$ the semantic value $X$ would have had in the actual world, given some possible meaning. (MacFarlane [2000: 6])

Being true in all models seen in accordance with (INT) and (MRA) just is being true under all I-PSIs. So we must distinguish the claim that Tarski's account is flawed from the claim that model theory is. It is what the model theory represents on the Tarskian approach which does not make for a plausible account of logical truth and consequence.

\subsubsection{Alternative Formulation of the Source of Tarski's Fallacy}

Accepting Priest's comments, which we looked at above, we saw that Tarski's account can, under certain conditions, guarantee that if the premises of a valid argument are in fact true, then so is the conclusion. This is a guarantee of actual truth preservation, and as I stated there, even if we were to accept that these conditions obtain, this kind of guarantee would still be insufficient to make sense of our practice of talking about, 
and using, valid arguments. In this section I want to make this clear by pointing out that if we accept the model-theoretic account relying on (INT) and (MRA), we cannot guarantee that for a valid argument with false premises, had it been the case that the premises were true, the conclusion would be true too. Nothing in the model-theoretic definition of logical consequence will be able to convince an agent of error, who believes that were the premises of a given valid argument true, the conclusion would still be false, unless the premises are already true. On the account in question, therefore, we are left without any explanation as to why logic, so to speak, works in counterfactual circumstances, something which we clearly do take logic to do.

Suppose that both agent $\mathrm{A}$ and agent $\mathrm{B}$ accept that an argument is valid if and only if it is truth preserving in all models seen in accordance with (INT) and (MRA). They therefore accept that the following argument, which is just an instance of modus ponens, is valid.

(P1) If unicorns exist, they belong to the equidae family.

(P2) Unicorns exist.

(C) Therefore, unicorns belong to the equidae family.

A and B agree that the argument is valid, so since they accept the Tarskian account they both believe that the argument preserves truth. Let us assume that they do so, not implausibly, because they both do not believe that unicorns exist. B, however, has a strange doubt. B doubts that had it been the case that if unicorns exist they would belong to the equidae family and also that unicorns exist, then it would be the case that unicorns belong to the equidae family. This, of course, is an extremely strange thing to doubt, and the reason is clear: the argument is just an instance of modus ponens, so of course it is valid, and consequently it could not be the case that (P1) and 
(P2) were true while (C) was false. This is our standard understanding of a logically valid argument.

But suppose B really does doubt this. What can A say to make him realize his mistake? They both agree that the argument is actually truth preserving, because they both think that there are no unicorns. Hence, pointing to the intended model, as we defined it above, will not help. A could point to all models in which both (P1) and (P2) are true and point out that (C) is true in all of these too. ${ }^{52}$ But, of course, this does not make B realize his mistake. All these models represent the actual world with different interpretations of the non-logical terms. All B should be convinced of is that no matter how the non-logical terms are interpreted the argument will actually preserve truth. But this is perfectly consistent with B doubting what he does.

Only insofar as A were to point to the members of the domain and say "look, suppose these are the unicorns and these are the members of the equidae family ...", does it seem that B ought to change his mind and accept that it cannot be the case that $(\mathrm{P} 1)$ and $(\mathrm{P} 2)$ are true while $(\mathrm{C})$ is false. But, and this is of course the point, $\mathrm{A}$ is not warranted in such an assertion from the perspective of his favored account of consequence. What $\mathrm{A}$ is effectively doing is describing a representation of a circumstance to B where there are unicorns, but no model, on the current approach, rightfully represents that. There are no unicorns, and A is only entitled to claims like the following: “look, suppose that 'unicorn' really meant horse, then can't you see that ...' But as noted, no claim of this form should force B to change his mind.

I take the moral of this little story to be the following:

- B does make a mistake.

- If we accept the model-theoretic account of consequence which sees the models

\footnotetext{
${ }^{52}$ Of course, we are speaking intuitively here. (P1), (P2), and (C) are not formal sentences and so are not true or false in models.
} 
in accordance with (INT) and (MRA) we cannot explain, using this fact only, that B makes a mistake.

Together, I take these two to entail that the model-theoretic account of consequence relying on (INT) and (MRA) does not provide an analysis of consequence in the sense of telling us what logical consequence consists in. But even though we cannot explain, from the account alone, that he makes a mistake, accepting the account does not entail that he does not make a mistake. That is, the extensional adequacy of the account cannot be challenged using this observation. There may be facts external to the account which can explain why B makes a mistake.

So far I have simply been assuming that B makes a mistake in thinking that it could be the case that $(\mathrm{P} 1)$ and $(\mathrm{P} 2)$ are true while $(\mathrm{C})$ is false. I think this is the only way to make sense of the practice surrounding valid arguments, how we speak about them and the use to which we put them.

We never find ourselves in a counterfactual circumstance, only actual circumstances, and since the model-theoretic account which sees models in accordance with (INT) and (MRA) can guarantee that an argument is actually truth preserving do we really need the stronger claim? The first thing to note is that this depends on the claim that definitional and epistemic order do in fact come apart, since there must be a way for us to know that an argument is valid independently of knowing that it fits the definition, if we are to use valid arguments to infer conclusions. We have argued that this is not very plausible.

If we accept the account relying on (INT) and (MRA), it turns out that logical necessity, understood as the "necessity" with which a logically valid argument preserves its truth, is an extremely weak notion. If a sentence is logically true, and hence logically necessary, it means only that there could be no language-respecting the 
meanings of the logical terms - in which the sentence expresses something which is actually false: that is, there are no interpretations of the non-logical terms under which the sentence expresses something which is actually false. ${ }^{53}$ Conversely, if a sentence is logically possible it means nothing more than that the sentence can be made to express something true in a language suitably similar to the original language, a language where the non-logical terms receive different interpretations. This, I submit, is not how we think about logical possibility and logical necessity.

The problem is that logical necessity, understood in the above fashion, is undefined over non-actuality (See Shalkowski [2004: 66ff], who also makes this point). Consequently reductio arguments do not show what we have been taking them to show: that the world could not possibly be as the assumption in the reductio says it is. All a reductio argument can show is that the assumption is not actually true and, in addition, that there is no suitably similar language in which the words used to express it can be used to express something true.

[E]ven if one successfully demonstrated that God exists and Evil exists constitute an inconsistent pair of propositions, in the available and very weak sense that not both of them are true, then no conclusions whatsoever about whether they are both true in other ways the world might have been would be thereby warranted. An atheist might be content to show only that God exists is false because Evil exists is true, but that was not the intent of the logical problem of evil. The strategy was to arrive at a conclusion about the actual world by going through a subsidiary conclusion about

\footnotetext{
${ }^{53}$ As we have already explained, Tarski defines logical consequence using the notion of satisfaction, which is, in essence, replacing the designations of the non-logical terms with other objects of appropriate type. We can therefore think of this as giving rise to a series of languages, each containing the same original sentence, where the meaning of this sentence differs in accordance with the objects that are designated by the non-logical terms, but where the logical terms retain their interpretations. When a sentence is logically necessary, it means that the sentence cannot express an actual falsehood in any of these hypothetical languages.
} 
impossibility. (Shalkowski [2004: 78])

Here we see Shalkowski pointing out what we have discussed above. We see our notion of logical consequence, when employed in reasoning to conclusions, as something which extends beyond the actual world: we $d o$, or at least the ones who believe the argument is sound, take the problem of evil to show that God exists and Evil exists cannot possibly be true at the same time. And though an atheist, as Shalkowski points out, would probably be content with just showing that God exists is false, this is just not how we think of the matter.

Similar points, of course, apply to counterfactuals. Assuming that a counterfactual is true when the consequent is logically entailed by the antecedent and some other background information, a true counterfactual would not tell us about how the world would be were certain other things such and such. It would give us information only about suitably similar languages and what would have to express a truth in those language, on the assumption that certain other things did.

\subsubsection{Dependent on Logical Constants}

At this point let us briefly consider a rejoinder that the proponent of (INT) and (MRA) might put forth, which is also considered by Etchemendy [2008: 269ff]. The proponent might point out that what the above criticism does not take into account are the properties of the logical constants. In a way, this rejoinder can be seen to be rather pointless from the beginning; after all, the fact that we are able to push these points without mentioning any of the properties of the logical constants must mean that their properties are irrelevant for these points to go through. But let us nevertheless entertain, for a while, the idea that the account can be salvaged by pointing to the special properties of the correct logical constants.

$[\mathrm{C}]$ onsider the following argument form: 


$$
\begin{aligned}
& P(a) \wedge Q(a) \\
& \neg P(b) \\
& \text { So, } P(c) \rightarrow Q(c)
\end{aligned}
$$

This is obviously not logically valid: from premises of the indicated form we are in no way justified in inferring the corresponding conclusion. Now it happens that there are non-truth-preserving instances of this argument form. But notice that this is not guaranteed by any features, global or local, of the truth-functional connectives appearing in the argument. For there would not be any non-truth-preserving instances if the world contained only two objects, or if all the objects in the world fell into two indistinguishable types. [2008: 269-270]

What Etchemendy is pointing out is that there are no features of the logical constants, which in this case are negation, conjunction, and implication, which secure that the argument form has non-truth-preserving instances. It is an easy exercise to show that the above inference only has countermodels with $|D|>2$, so the argument would be valid if there were only two objects. Hence, in this case the logical constants would not be able to guarantee that the argument is truth-preserving, as this is guaranteed only by an empirical fact that could have been different. We therefore have no reason to think that the logical constants are in general capable of securing a modal profile of valid arguments.

However, Etchemendy's second claim, that there would be no non-truth-preserving instances of this argument if all the objects in the world fell into two indistinguishable types, is false. If the proper denotations for $P$ and $Q$ are simply sets, something which is assumed by Tarski's account and the model-theoretic definition, it does not matter that there are only two indistinguishable types of objects. All that matters is that we can 
form the following sets: A set, $S(P)$, which includes an objects $o_{1}$ and $o_{2}$ and excludes $o_{3}$, and a set, $S(Q)$, which includes $o_{1}$ and excludes $o_{2}$ and $o_{3}$. Consider the sequence which assigns $S(P)$ and $S(Q)$ to the "variables" $P$ and $Q$, respectively, and $o_{1}, o_{2}$, and $o_{3}$ to the "variables" $a, b$, and $c$, respectively, in the above "sentential functions". ${ }^{54}$ It is clear that this sequence is a model, in Tarski's sense, of the premises but not of the conclusion; hence, the argument does have non-truth-preserving instance quite independently of whether the three objects fall into two indistinguishable categories. What matters is that we can define the given sequence. Of course, we may encounter problems when confronted by the epistemic question as to how we know which sequence is the sequence in question, but this should be irrelevant for the purposes of defining the sequence.

This, of course, does not show that the logical constants can secure a modal profile. It only shows that Etchemendy's second reason is not accurate. The point that had there been only two objects, then there would have been no non-truth-preserving instances still stands. And even if we can secure that not only are there infinitely many objects, there are necessarily infinitely many objects, we are still left with the problem, discussed in the previous section, that logically valid arguments do not extend beyond the actual world on a definition which endorses (INT) and (MRA). And this point is independent of any features that the chosen logical constants may possess.

\footnotetext{
54 The quotation marks serve to indicate that $P, Q, a$, and so on, are not variables; they are formal terms in the formal sentences, $P(a) \wedge Q(a)$, and so on, which are not sentential functions. What we mean by the above is the variables corresponding to the formal terms and the sentential functions corresponding to the formal sentences.

Another way of putting the point, on the level of model theory, is to point out that the following is a countermodel, $M=\langle D, I\rangle$, s.t. $D=\left\{o_{1}, o_{2}, o_{3}\right\}, I(a)=o_{1}, I(b)=o_{2}, I(c)=o_{3}, I(P)=\left\{o_{1}, o_{2}\right\}$, and $I(Q)=\left\{o_{1}\right\}$. This countermodel remains a countermodel even if we assume that the three objects fall into two absolutely indistinguishable categories.

Of course, there may be a problem here with individuating which object is $o_{1}, o_{2}$, and $o_{3}$, respectively, when presented with this countermodel. That, however, is a different question.
} 
As Etchemendy points out: enquiring about the correct division between logical and non-logical terms when the model theory relies on (INT) and (MRA) is just asking when the definition happens to capture an informal notion where the truth of the premises does guarantee the truth of the conclusion. And the answer, as we shall look at in the next chapter, is that it does so when the model theory is also acceptable from a representational perspective. 



\section{PART II}

In the previous chapter we looked at Etchemendy's criticism of Tarski's account of logical consequence, an account we found to translate into a contemporary modeltheoretic account of consequence which sees the models in accordance with (INT) and (MRA). As we saw, this account cannot be sustained. If truth preservation in all such models is what it means for an argument to be logically valid, then we have no assurance that logically valid arguments will work in non-actual circumstances. We consequently have no assurance that logic can tell us anything about how the world would have to be under certain false assumptions. Yet, we clearly do act and speak as if logically valid arguments can tell us something about the world under false assumptions. Logical possibilities, those in which a logically valid argument preserves truth, are more than

mere representations of the actual world with different interpretations of the non-logical terminology.

However, as we also noticed, Etchemendy's criticism is not against model-theoretic characterizations of logical consequence, but against a particular view of what the models of the model theory represent. In the present chapter we will look at how to properly understand model-theoretic accounts of logical consequence according to Etchemendy. This will help us draw an even sharper contrast between Etchemendy's positive views and the views discussed in chapter 1, and thereby make sense of Etchemendy's claim that his view of model theory cannot count as an analysis either.

After we have presented Etchemendy's positive views we will discuss the various 
features which the defined relation of consequence will possess, and how we can make sure that it does so. In relation to this, squeezing arguments become relevant. We will consequently have to say something about these.

\subsection{Etchemendy's Modeling Perspective}

According to Etchemendy's own view of model theory, when used to explicate logical consequence, it is absolutely essential that we see the models as representing ways the world could have been. For logical consequence the models are meant to represent logically possible ways the world could have been, or, if you will, logical possibilities. In essence, then, we are using the model theory to capture the intuition that a logically valid argument just means an argument that preserves truth in all logical possibilities.

The guiding idea of the representational view of model theory is simple, and in fact widely held, though not widely articulated. The idea is this. The set-theoretic structures that we construct in giving a model-theoretic semantics are meant to be mathematical models of logically possible ways the world, or relevant portions of the world, might be or could have been. They are mathematical models in a sense quite similar to the mathematical models used to study, say, the possible effects of carbon dioxide in the atmosphere, only they are used to study semantic phenomena, not atmospheric, and specifically to characterize how variations in the world affect the truth values of sentences in the language under investigation. The main difference is that in model theory we normally use discrete mathematics rather than the continuous mathematics used in physical modeling, though one can eas-

ily devise languages where discrete tools do not suffice. I called this view of model theory "representational" because the set-theoretic structures are 
seen as full-fledged representations: models of the world. (Etchemendy

[2008: 287])

Here we have Etchemendy describing his representational view in broad outline. The important feature, of course, is that models represent possible circumstances, hence (MRA) is rejected in relation to the models. Models are mathematical models of ways the world could logically have been. ${ }^{55}$ This is important. The models are settheoretic structures of some kind that represent ways the world could have been. For Etchemendy, models consist of actual objects, and what gives these objects the capacity to represent are the ways in which they can combine to form structures. Though he does explicitly state that models consist of actual objects, besides explicitly endorsing that they might be abstract, Etchemendy does not really qualify this claim. One might wonder to what extent an object can be abstract but still actual. Numbers, assuming they are abstract, are presumably such, as are pure sets. However, there are less clear cut cases. Are unicorns abstract and actual, is the seventh son of Kripke abstract and actual, is the round square abstract and actual? It might seem an idle point, but insofar as there are valid arguments or logical truths containing these expressions, we would want to know how the models manage to represent the relevant possibilities. And it is not straightforward how they manage to do so. If these examples, or others like them, truly are non-actual and so cannot play the role of the objects of a given domain, the question is how any model comes to represent circumstances involving unicorns or the like. We will come back to these issues in chapter 3 .

What matters, on Etchemendy's view, is that we have a representation of each and every way the world could have been.

\footnotetext{
55 Note that there are two different uses of the term 'model' here. One refers to the actual model, a structure which is given by a domain and an interpretation, $\langle D, I\rangle$. The other refers to that which such a model is a model of. For example, for interpretational semantics, a model models an interpretation of the non-logical terms, whereas for representational semantics, a model models a representation of how the world could be.
} 
According to the representational view, our goal in constructing a semantics is to devise a class of models that represents all logically possible ways the world might be that are relevant to the truth or falsity of sentences in the language, and to define a relation of truth in a model that satisfies the following constraint: a sentence $\varphi$ should be true in a model A if and only if $\varphi$ would be true if the world were as depicted by A, that is, if A were an accurate model. [2008: 287]

The goal of a representational semantics is to have a representation of each and every logically possible way the world could have been, and have an adequate definition of truth in a model. As Etchemendy goes on to explain, this secures in a very straightforward manner that a sentence which is true in all models is logically true. If the goal of the representational semantics has been met, then a sentence $\varphi$ is true in all models just when it is true in all logically possible ways the world could have been. Here we see how we are simply exploiting the truism that a logically valid argument is one such that it preserves truth in all logically possible circumstances, and similarly for a logical truth. Of course, nothing so far has been said about how a model represents a logically possible way the world could have been and how we know that all and only logically possible ways the world could have been are represented. This question is important and depends crucially on what a logical possibility is. As pointed out above, if our language contains expressions that do not have an actual referent, then we will need a theory of how something actual can represent something non-actual.

However, assuming that we have managed to construct a model theory in accordance with the guidelines, we can be sure that only genuinely valid arguments are declared valid. Etchemendy goes on to state that for this reason, a representational model theory "does not give us an analysis of the logical properties since the logical notions are presupposed from the very start, in the criteria by which we assess our models" 
[2008: 288]. This sets the representational approach apart from an approach that defines logical consequence as truth preservation in all models seen in accordance with (INT) and (MRA). Such an approach would, if it were successful, manage to analyze the notion of logical consequence in terms of other more well-understood notions that do not presuppose the notion of logical consequence; it would tell us what logical consequence consists in in terms of more basic entities. Etchemendy is here claiming that representational semantics cannot deliver such an analysis. This claim requires some elaboration.

\subsubsection{Not An Analysis}

The second issue is the issue of analysis itself: If Tarski's analysis worked, it would be a genuine analysis, in the sense that it characterizes the logical properties in terms that do not presuppose those very same properties. As I mentioned earlier, representational semantics gives us no such analysis, since the logical notions are used to assess the class of models devised for the semantics. Each model is meant to depict a logical possibility; no two are logically consistent; and the "sum" of the models is logically necessary- that is, every semantically relevant possibility is represented. This has a consequence that some may find disappointing, though it should hardly be surprising. We cannot look to model-theoretic semantics to answer the most basic foundational issues in logic. For example, if we have serious doubts about whether the principle of excluded middle is a logical truth, the classical semantics for propositional languages will not provide an answer. For the same intuitions that suggest that it is a logical truth are used in defining the class of structures - truth value assignments - that are employed by this semantics. [2008: 294, my emphasis] 
Suppose an analysis of a phenomenon consists in giving a characterization of the phenomenon in terms of concepts that do not presuppose the phenomenon itself. Then Etchemendy is making the following claims:

a) If a model-theoretic account relying on (INT) and (MRA) worked, it would count as an analysis of logical consequence.

b) The representational account of model theory cannot count as an analysis of logical consequence.

We have seen how an account of model-theoretic consequence in accordance with (INT) and (MRA) characterizes logical consequence in terms of the notions of truth and satisfaction; two notions that do not presuppose the very notion of logical consequence.

To see why this is not the case for the representational semantics, we may point out again that representational semantics relies on the truism that being logically valid is being truth preserving in all logical possibilities. But whatever a logically possible way the world might have been consists in, this notion is presupposed by the model theory in that we have managed to represent each and all of them. An analysis of what it is to be a logically possible way the world could have been does not come from the model theory. Using an analogy of MacFarlane's claim about the interpretational model-theoretic account (MacFarlane [2000]), the point is this:

\section{Truth preservation in all models}

is a model of

\section{Truth preservation in all logical possibilities}

is conceptually equivalent to 


\section{Logical consequence}

The problem here is that the last step is just making explicit what we mean when we use the concept logical consequence; it is, using the term used earlier, a truism. That is not to say that there is nothing illuminating going on here, but only that logical consequence cannot be analyzed by the notion of logical possibility. This, of course, is not to say that the notion of a logical possibility cannot be further analyzed in terms of other more basic notions, and that we can in that way have an analysis of logical possibility, and consequently, logical consequence. The point about this approach not being an analysis is that the model theory is applied to model the relation given by (R). There might very well be a way to analyze, in terms of more basic notions, the notion of logical possibility, but this analysis must come from elsewhere than the model theory applied to model (R). In the case of the Tarskian account we had the following:

\section{Truth preservation in all models}

is a model of

\section{Truth preservation under all I-PSIs}

is conceptually equivalent to

\section{Logical consequence}

And as we saw in the first chapter, it is exactly this last step that makes it so that we are not dealing with an analysis. Truth preservation under all I-PSIs is not conceptually equivalent to logical consequence.

As we pointed out above, we are not denying that there is a way to analyze the notion of logical possibility in terms of another notion, but we should point out that 
this analysis does not result from the application of a model theory. An application of a model theory to demarcate the logical consequence relation cannot count as an analysis. If it did it would eventually either be circular, following $(\mathrm{R})$ above, or, the analysis would ultimately rest on a mistake, following (I), and so not be an analysis in the first place. Now, I am not claiming that there is no possibility that modeltheoretic tools are ultimately able to provide us with an analysis of the notion of a logical possibility in the requisite sense. But, the model theory applied to model the relation $(\mathrm{R})$ would presuppose this analysis, and so that very application would not be the analysis of logical consequence. Perhaps the point here is rather pedantic, and in any case, it is a small point, perhaps pertaining rather to what we should be willing to call 'an analysis proper'. I am not denying that one can give a structural analysis of a (logically) possible way the world could have been which is a reduction to structural entities also found in models. But, properly speaking, such an analysis would not be part of the model theory, but rather we should say that the model theory is relying on it.

It is important to note, however, that this does not mean that there is nothing interesting about model-theoretic definitions of consequence. Although on Etchemendy's view it does not tell us what logical consequence consists in, it still illuminates the target phenomenon:

Does this mean that model-theoretic semantics, construed representationally, provides no illumination about the logical properties of the languages studied? Not by any means. We can see how it illuminates these properties both concretely and abstractly. Concretely, a well-designed semantics shows us how the truth values of sentences in the target language vary as the non-linguistic facts represented by the structures vary, and accordingly explains persistent patterns of truth values that emerge due to the seman- 
tics of the sentences. Logical truth and logical consequence are just two such persistent patterns. ... [A]ssuming a clear understanding of the states of affairs depicted by our models, the semantics shows precisely how the logic of the language arises from the meanings of its constituent expressions, modulo any basic logical assumptions incorporated into the models themselves. For example, the classical semantics for propositional logic may not provide a fully grounded explanation of the principle of excluded middle, but it does explain why, given this basic assumption, a complex sentence like $\neg(P \wedge(\neg P \vee(Q \wedge R))) \vee Q$ is necessarily true. This provides illumination of a very real sort. [2008: 294]

Model-theoretic semantics, viewed representationally, is illuminative. If we understand how a model represents, then such a semantics illuminates how the truth values of sentences depend on the facts, and we can see how arguments that are truth preserving in all models are truth preserving no matter what.

The illumination, however, is modulo some basic assumptions. Deciding on the behavior of the logical constants in all models just is deciding on the logically valid arguments and logically true sentences. A prime example is modus ponens, which is valid in a classical semantics because $\rightarrow$ (if... then...) is given the semantic clause that it is. However, though the explanation of the truth of basic assumptions, such as the law of excluded middle, on which we base the model-theoretic account of consequence, cannot be found in this account itself, the model-theoretic account can still explain why given these assumptions, complex arguments and sentences are logically valid and logically true, respectively.

Etchemendy states the following in a footnote:

Note, by the way, that my own explanation of the validity of modus ponens 
is semantic, not modal. I am no particular fan of modal characterizations of consequence, and thought I had made that clear in [The Concept of Logical Consequence]. In spite of that, some commentators seem to have concluded that I identify logical truth with necessary truth and logical consequence with necessary truth preservation. Nothing could be further from the truth. [2008: 271]

This remark appears quite puzzling at first. Etchemendy's criticism does seem to rely on the fact that the valid arguments on Tarski's account lack a modal profile, but here Etchemendy is stating that he is no fan of modal characterizations of consequence. This need not necessarily bother us, of course, since Etchemendy's criticism surely is not that Tarski's account is not couched in modal terms, but that no modal profile for the valid arguments can be seen to follow in terms of Tarski's definition. The criticism is not that a theory of consequence must be couched in modal terms, but that a modal element must follow from the definition. Why does Etchemendy's own account not count as a modal characterization of consequence by Etchemendy's lights? After all, the models represent possible ways the world could have been. The key to understanding this, I submit, is to focus on the fact that the models represent logically possible ways the world might have been, and what is logically possible, and thereby also what is a logically valid argument, is at bottom a semantic phenomenon; though as an upshot of this, the logically valid arguments will possess a modal profile. We will return to this briefly below.

\subsubsection{Interpretational Character}

When we study the logic of a language, we are generally interested in the logic of only some of its expressions. In propositional logic we are interested in the truth-functional connectives; in first-order logic we additionally 
focus on the quantifiers and identity; in modal logic we add necessity and possibility; in epistemic logic, knowledge and belief; and so forth. Because we focus only on some of the expressions in the language, the semantics of the remaining terms can be treated differently from those whose logic we aim to explicate. I will say we treat them "categorematically" for lack of a better term. [2008: 288]

Etchemendy is here introducing his notion of a categorematic term, a term whose meaning we choose to abstract away from when setting up our model-theoretic semantics. The genuine consequence relation will emerge when the semantics of all terms is taken into account, but sometimes we are interested only in the logic of specific terms. This happens for instance when we set up a model theory for a propositional language; we are interested only in the arguments whose validity depend upon the truth functional connectives, and we therefore abstract away from the exact meaning of the atomic sentences. Only the minimal semantic behavior common to expressions of the same semantic category, in this case atomic sentences, is taken into account. For atomic sentences this is a truth value: this is the minimal semantic behavior common to all atomic sentences. To know the truth value of an atomic sentence is sufficient to determine the truth value of a more complex sentence (assuming that the truth values of other constituent atomic sentences are known). As Etchemendy points out, treating some terms categorematically does not mean that there is no interesting semantic behavior to terms that are treated categorematically. ' $a=a$ ', for example, is semantically very interesting in that it cannot be false, but that is ignored by a propositional modeltheoretic semantics, where this sentence is afforded the treatment of a propositional variable, and can consequently be true in some models and false in others.

Treating some terms categorematically naturally brings a change in the perspective on the models, though Etchemendy stresses that it is a minor change. 
How does the categorematic treatment of certain expressions affect the representational view of model theory? Well, not much at all. Where earlier we said that every model is meant to represent a logically possible configuration of the world, we now need to add a qualification to handle expressions treated categorematically. Each model represents a semantically relevant circumstance for at least some expressions in those categories - or, if you will, for some interpretations of the expressions so treated. This is a minor change, but it is potentially confusing because there are now two dimensions of variation. Structures still represent possible circumstances - this is the important dimension of variation-but our decision to treat some expressions categorematically introduces a second dimension, since these expressions receive uniform treatment in spite of potentially significant variations in their meanings. ...

From the representational standpoint there is only one significant effect of the categorematic treatment of expressions. If we do not treat any expressions categorematically and our semantics meets the representational guidelines - that is, if every logically possible circumstance is represented by some model and our definition of truth in a model is correct - then we can be sure that all and only logically valid arguments of the language are declared valid. [2008: 289]

Etchemendy is here pointing out that there are now two dimensions of variation: a dimension of representations, which comprises the various representations of how the world could logically have been, and a second dimension, having to do with assigning a denotation to the terms that are treated categorematically. It is noticeable that Etchemendy points out that the second dimension of variation is due to these terms receiving a uniform treatment, since a uniform treatment seems to be the opposite of 
variation. Here is how we can understand this claim: the decision to treat some terms categorematically introduces an interpretational aspect to the model theory. Because the model theory ignores any particular meaning that the categorematic terms have, but their minimal semantic behavior is still needed to determine truth in a model for all sentences of a language, we see each model as providing an interpretation - as interpreting the term in question - by assigning it a value from the range determined by the minimal semantic behavior common to all expressions of that category. But this is just to say that the model, besides representing a possible circumstance, interprets the categorematic terms. According to Etchemendy's view of model theory, then, a model theory for explicating logical consequence is both interpretational and representational. But it is interpretational only insofar as we choose to treat some terms categorematically. As we shall later look at, such a decision is not in principle forced upon us, though there may be practical restrictions on the modeling capacities of our framework which forces some categories of terms to be treated categorematically.

It is natural to see the categorematic terms as non-logical constants. Of course, there is nothing wrong with calling these 'non-logical constants', but one should bear in mind that there are two fundamentally different ways to give content to the notion of a logical constant. On one such conception, the use of 'logical term' as opposed to 'non-logical term' is supposed to mark a fundamental difference; the logical terms possess qualities which the non-logical terms do not. Etchemendy does not believe in such a principled distinction.

On the other hand we can see the 'logical' versus 'non-logical' terminology as simply marking a distinction in our current treatment of the terms. On this view there is no principled distinction between the two classes of terms, but for reasons of interest we are currently allowing models to provide interpretations of one kind, whereas the interpretations of terms of the other kind are held fixed. On this conception, then, 
there is nothing wrong with running together Etchemendy's notion of a categorematic term and a non-logical term.

On both these conceptions a valid argument is one such that it is guaranteed to preserve truth in virtue of the logical constants. In this sense, we may also see Etchemendy as falling into the first category. A valid argument is one such that it is guaranteed to preserve truth in virtue of the logical terms; it is just that all terms are logical.

At this point, a note on terminology is in order. In Etchemendy [1990], Etchemendy refers to the model-theoretic view which results from Tarski's account as an interpretational semantics. As made clear in the first chapter, this is a model-theoretic account which endorses (INT) and (MRA). As should be clear from the discussion in chapter 1 , it is not really the interpretational aspect - that is, the fact that models provide interpretations - which is to blame for the failure of the account, but rather the fact that each model represents the actual state of affairs. For this reason it is perhaps confusing to criticize a model-theoretic semantics under the heading of interpretational semantics. Let us keep in mind that the target of Etchemendy's criticism is what we might call purely interpretational model-theoretic semantics, a semantics that sees variations in models only as variations in interpretations from the perspective of the actual world. In the remainder of the present thesis an interpretational semantics is to mean a semantics which has an interpretational aspect to it. It need not therefore be purely interpretational. When we want to speak of a model theory as that which is the target of Etchemendy's criticism, we will speak either of a model theory which sees the models in accordance with (INT) and (MRA) or as a purely interpretational model theory.

In the next section we will concern ourselves with the question as to what it is that a model is meant to represent, that is, what is a logically possible way the world could 
have been? $?^{56}$

\subsubsection{A Logically Possible Way The World Could Have Been}

Etchemendy does not explicitly say anything about what he takes a logically possible way the world could have been to be. Of course, if we know that our models represent all and only such possibilities, then we can be sure that all and only genuinely valid arguments are declared valid by our semantics, given the assumption that no terms are treated categorematically. However, this does not tell us anything about what a logically possible way the world could have been $i s$; it does not tell us anything about what our models are meant to represent. Such a story is needed to secure that our semantics meets the representational criteria; if we do not know what our models are meant to represent how can we judge that they do so adequately?

At this point it will be instructive to compare Etchemendy's comments on his positive views of model theory with a theory of model-theoretic consequence which clearly endorses a representational stand, but also endorses a specific view of what the model theory represents. Such a theory is provided by Shapiro [1998, 2005].

Shapiro [1998] starts by noting, as we saw Tarski do in his original article, that the concept of logical consequence, as it is found in everyday language, is not sharp. This suggests that there is no such thing at the intuitive, or the informal, concept of logical consequence which it is the job of the formal definition of consequence to correctly capture.

Before we can ask whether a rigorous definition captures the concept of logical consequence, we must first sharpen the informal notion to a point where the question of whether this very concept is captured makes sense. There are various levels involved.

\footnotetext{
${ }^{56}$ How a model is to represent such a possibility is an important question as well, though it is one that will primarily be investigated in the next chapter.
} 
As Smith [2011] puts it:

There are three conceptual levels here:

(1) We start with a rather inchoate jumble of ideas of validity[.]

(2) We can sort things out in various directions. Pushing some way along in one direction (and there are other ways we could go, equally well rooted - ask any relevantist!), we get an informal, still somewhat roughand-ready classical notion of validity-in-virtue-of-form.

(3) Then there are crisply defined notions like derivability-in-your-favouritedeductive system and the modern post-Tarski notion of validity.

The move from the first to the second level involves a certain exercise in conceptual sharpening. [2011: 28]

Smith uses the distinction to drive home a point about squeezing arguments; that squeezing arguments cannot prove the coextension of concepts belonging to step (1) and (3), respectively. Once the division has been made clear this seems obvious, but the problem is that this point is easily overlooked. If there isn't just one informal concept of logical consequence, then there can naturally not be any argument that a given definition captures it.

Accepting this picture, there are three steps involved in the prospect of delivering a definition of logical consequence. In moving from step (1) to (2) we sharpen the intuitions about our concept as to make it one coherent, but perhaps still informal, notion of consequence, and in moving from step (2) to (3) we attempt to show that a rigorously defined concept captures the informal concept given in step (2). Once again we can see how Etchemendy's criticism is not a criticism of model theory. The problem is not that the model theory is not able to capture the notion of being (actually) truth 
preserving under all interpretations of the non-logical terminology. This result follows from Tarski's work [1956a, 1956b]. The problem, rather, is that being (actually) truth preserving under all interpretations of the non-logical terminology is not a legitimate sharpening of our intuitions concerning logical consequence. It is this claim that is the central claim of Etchemendy's criticism of a purely interpretational account of consequence. A sharpening of our intuitions to that of being (actually) truth-preserving under all interpretations leaves out the crucial features of logical consequence.

Shapiro suggests that a model-theoretic account of consequence is meant to capture what he calls a blended sense of logical consequence, a sense in which variations of the meanings of the non-logical terms as well as representations of possible circumstances play a role. The notion of consequence, for natural languages, which Shapiro sees model theory as attempting to capture is the following:

Let us say that a sentence $\Phi$ (in a natural language) is a consequence of a set $\Gamma$ of sentences in a blended sense if it is not possible for every member of $\Gamma$ to be true and $\Phi$ false, and this impossibility holds in virtue of the meaning of the logical terms. In the terminology of possible worlds, $\Phi$ is a logical consequence of $\Gamma$ in this blended sense if $\Phi$ is true in every possible world under every reinterpretation of the non-logical terminology in which every member of $\Gamma$ is true. (Shapiro [2005: 663])

This blended sense, which Shapiro is advocating, incorporates both the tenets of necessity and formality which we saw in the previous chapter. Logically valid arguments preserve truth necessarily, and they do so in terms of the meanings of the logical terms. There are a few things to note about Shapiro's theory. First off, model theory is being proposed as a way to model a notion of logical consequence. This approach 
is sometimes referred to as the 'logic-as-model' approach (Shapiro [2001: 160]). Secondly, the blended notion is but one of many legitimate notions of consequence. As a prominent example of another legitimate notion, Shapiro mentions the deductive notion of consequence. Again, $\Phi$ and $\Gamma$ represent natural language sentences and sets of sentences, respectively.

(Ded) $\Phi$ is a logical consequence of $\Gamma$ if there is a deduction of $\Phi$ from $\Gamma$ by a chain of legitimate, gap-free (self-evident) rules of inference. [2005: 660]

This notion of logical consequence is the notion we try to model when using a proof theory. But neither of these is more important than the other.

I submit that model-theoretic consequence and deductive consequence each correspond to a different intuitive notion of logical consequence, namely the blended notion and (Ded) respectively. [2005: 667]

We here see Shapiro endorsing the idea that there are more than two intuitive notions of consequence. In fact, Shapiro lists nine different ways of giving content to the claim that a sentence follows logically from a set of sentences (Shapiro [1998: 132ff]). There are multiple intuitions concerning logical consequence, and they are not all sharp or clearly demarcated from one another. However, we can sharpen the intuitions to a point where these notions can be modeled with rigorous methods. Such is the case with model theory that models the blended notion above.

If the model theory is to capture a notion of necessary truth preservation under all interpretations of the non-logical terminology, the model theory must somehow have both a representational and an interpretational aspect to it. One might therefore wonder whether this blended notion is the same notion which we seek to capture when applying Etchemendy's modeling perspective. 
It is clear that Shapiro's notion of a possible world just is a genuinely possible world, what we normally refer to as a metaphysically possible world. ${ }^{57}$ This, one might worry, is a substantial price to pay to have a working theory of logical consequence, since the debate concerning metaphysically possible world is replete with thorny metaphysical issues. However, as we shall see below, Shapiro presents evidence to the effect that under certain assumptions about the terms treated logically, we need only consider various cardinalities of the underlying domains in order to secure that the notion of truth preservation in all metaphysically possible worlds is captured by the model theory. We will look at these arguments below.

It is unclear whether this is a notion which Etchemendy would endorse. The first point to note is that if all terms are treated as logical terms - something which, according to Etchemendy, will have to be done to capture the genuine consequence relation- this seems to just collapse to necessary truth preservation. If logical consequence is truth preservation in all metaphysically possible worlds under all interpretations of the non-logical terminology, then denying that there is anything to reinterpret would collapse logical consequence into truth preservation in all metaphysically possible worlds: a purely modal characterization, something Etchemendy explicitly states that he does not endorse (Etchemendy [2008: 271, n2]).

I think the clue to understanding this is to focus on the semantic part of Etchemendy's thesis. It is in virtue of the semantics of the terms that an argument preserves truth necessarily.

(sem) $\Phi$ follows logically from $\Gamma$ if and only if in virtue of the semantics of all the terms occurring in $\Gamma$ and $\Phi$ it is impossible for $\Gamma$ 's sentences to be true while $\Phi$ is false.

This, of course, entails that a valid argument could not possibly have true premises and a false conclusion, but this, in the light of what we looked at in the previous chapter,

\footnotetext{
57 That is not to say that these must be understood in realist terms, nor is it to deny this.
} 
should be a welcomed consequence. So while this characterization of consequence is certainly modally involved, the notion so obtained is a semantic notion: it is in virtue of the semantic features of the language that a valid argument preserves truth necessarily. Appropriate changes to the semantics of the terms of the language would yield changes in the extension of the consequence relation, but if there are such things as possible worlds, then, presumably, whatever goes on in them-i.e., whatever is possible or impossible - would be left unaffected by such changes. ${ }^{58}$

This sits well with Etchemendy's explanation of how we recognize that modus ponens is a valid argument:

The crucial feature of modus ponens is that we can recognize that all of its instances preserve truth without knowing the specific truth values of the sundry instances. My own view is that we recognize this by virtue of the meaning of the expression if...then and our knowledge of how the remaining constituents can contribute to the truth values of the premises and conclusion. (Etchemendy [2008: 268], my emphasis)

If we endorse something like the principle $(\mathrm{CON})$ given in the previous chapter, how we come to know that an argument is valid will be intimately linked, explicitly or implicitly, to the content of being a valid argument, that is, what validity consists in. Even should we not accept this principle in general, I do not think it would be far from

\footnotetext{
58 This way of making sense of Etchemendy's claim that modal characterizations of consequence are mistaken (Etchemendy [2008: 271, n2]), takes a purely modal characterization of consequence to be concerned with that which is expressed by the language in question. This way of putting things, a purely modal characterization of consequence is given at the level of propositions. But since propositions are that which is expressed by a statement given certain semantic features of the language, they do not themselves have a semantics, and consequently, the modal characterization is not semantic. However, taking into account the points we made in the introduction about statements derivatively standing in the consequence relation, even when the proper relata of logical consequence are propositions, it follows that this derivative notion of consequence would still be affected by the semantics of the language, as it would determine which statements express which propositions. I admit that I do not straightforwardly know how to make sense of Etchemendy's claim that his own characterization is semantic, not modal. If anything, it seems to be both.
} 
Etchemendy's spirit to posit that not only is this how we realize that an argument is valid, it is also what it means for an argument to be valid, as he stresses in multiple places that he sees logical consequence as a semantic phenomenon.

On Etchemendy's view, each model represents how the semantics of the terms determine the truth values of each sentence in the language for a given configuration of the world. This, again, is a semantic notion, but the 'can' which results from the totality of the underlying model theory in the formulation '... how the remaining constituents can contribute to the truth values of the premises and conclusion' is a genuine 'can': one that signals genuine possibility. Each model still represents a possible way that the world could have been which affects the truth values of the sentences of the language, and the ways in which a possible way the world could have been can affect the truth values of the sentences is given by two parameters: the possible ways that the world could have been, as represented by models, and the part of the semantics taken into account by each model. This, of course, leaves open the question of what it means for the model theory to take the semantics of a given term into account, that is, what does it mean to treat a term as a logical term and its formal counterpart as a logical constant? Unfortunately, Etchemendy never gives any positive account of this. We will look at this further in the next section.

If we accept this, we can once again see how we are not providing an analysis of the notion of logical consequence. $\Phi$ is a logical consequence of $\Gamma$ if the semantics of the constituent terms excludes the possibility of $\Gamma$ consisting only of true sentences while $\Phi$ is false. However, in setting up the model-theoretic semantics and judging it adequate, we are relying on the fact that we have managed to represent all semantic possibilities for the terms. Or in other words, we are simply stipulating what is semantically possible for the relevant terms, an explanation as to why this is the case must come from outside model theory. 
We see Etchemendy expressing this kind of view as early as 1988 :

Models are just abstract representations of the world, both as it is and as it might have been. To use a rather loaded term, models represent possible worlds. But no queer ontological commitment is involved in this representation; indeed the fact that models can represent things that don't actually exist is what makes them so very useful. [1988a: 95]

We here see Etchemendy endorsing the idea that models represent possible worlds. ${ }^{59}$ Exactly how they manage to do so will be the topic of the following chapter. For now, we may note that Etchemendy does endorse something like Shapiro's idea of the blended sense of logical consequence as that which the model theory is supposed to capture. But, as mentioned repeatedly above, the focus is on the semantics of the language, not on full blown representations of the world. Only insofar as the different circumstances which the models represent have an effect on the truth values of the sentences of the language, is the difference relevant from this perspective:

[I]f our models represent each semantically significant configuration of the world, and we carefully explain the truth conditions of our sentences in terms of these models, then the by-product of our semantics, the logically true sentences, will naturally hold true no matter what the world is like. A partial account of analyticity is a by-product of a good model-theoretic semantics. [1988a: 95-96]

We here see a clear precursor of the positive ideas Etchemendy expresses about model theory in Etchemendy [2008]. ${ }^{60}$ But the emphasis is still on the semantics; we are rep-

\footnotetext{
59 Though, of course, this is not endorsing any particular view of possible worlds. See also Barwise \& Etchemendy [1989: 217ff], where it is clear that models represent genuinely possible states of affairs (or parts thereof).

${ }^{60}$ Perhaps, in fact, 'precursor' is too strong a word. Perhaps this just is the positive view of model theory which Etchemendy expresses in the 2008 article.
} 
resenting semantically relevant, or significant, configurations, and the resulting logical truths are necessarily true given the semantics of the terms. On this account a logical truth is simply an analytic truth (though there is no commitment to all analytic truths being logical truths). An analytic truth is standardly taken to be a statement which is guaranteed to be true in virtue of the meaning of the involved terms. A prime example would be the statement expressed by 'All bachelors are unmarried'.

Now, there is a subtlety here which, though I will not dwell on it, is worth mentioning. It is often said that 'All bachelors are unmarried' is true in virtue of the meanings of the involved terms. This much is uncontroversial, but so is the claim that the statement expressed by 'James is a bachelor' is true in virtue of the meaning of the involved terms. It is in virtue of these meanings that the sentence 'James is a bachelor' expresses what it does, and so (partly) in virtue of these that the statement it expresses is true. What we mean to be conferring when claiming that the former statement is true in virtue of meaning is perhaps better put by claiming that the statement expressed by 'All bachelors are unmarried' is true solely in virtue of meaning of the involved terms, or that in virtue of the meaning of the terms, the possibility that 'All bachelors are unmarried' is false is ruled out. ${ }^{61}$ It is clear that the notion which Etchemendy speaks of in the above quote is a logically true sentence as one which is true no matter how the world is. The interesting concept is thus one of being necessarily true in virtue of meaning, or being true solely in virtue of the meaning of the involved terms. ${ }^{62}$

There is evidence to suggest that the interesting concept for Etchemendy is the notion of a sentence which, in terms of the meaning of its constituent terms, could not

\footnotetext{
${ }^{61}$ I do not mean to be conferring that anyone holds a theory of analyticity where an analytic truth is simply a statement that is true in virtue of meaning in the first sense, as this would make all true statements analytically true. Rather, I am pointing out that when we speak of analytic truths, we standardly have in mind the concept of being true solely in virtue of meaning. There are of course various ways of making this latter precise, but whether the 'solely' component is mentioned or not, it is, to the best of my knowledge, universally assumed.

${ }^{62}$ See Williamson [2007: ch. 3+4] for a discussion of the notion of analyticity in relation to, among other things, this mantra of 'being true (solely) in virtue of meaning'.
} 
be false. This much should be clear by our discussion of Etchemendy's view of model theory. This suggests that logical truths just are analytic truths.

I have no complaints about logical truth conceived as a form of analytic truth: a logically true sentence is one that's true thanks to the meanings or semantic functioning of certain special terms, those we've traditionally singled out as the logical constants of the language. The set of logical truths will thus be a subset, though perhaps not a proper subset, of the analytic truths of a language. [1988a: 98]

Etchemendy is here pointing to a difference between logical and non-logical constants in order to explain the difference between a logical truth and an analytic truth. But the difference is demarcated through nothing more than the fact that logicians traditionally have been interested in the former. So, even if we read Etchemendy here as having a distinction between two classes of terms in mind, it can hardly be said to be a principled distinction; at least insofar as it is a contingent fact exactly what terms have traditionally been counted as logical. I think the natural way to understand Etchemendy here is to see him as simply making the verbal and historical point that, as a matter of fact, through the history of logic focus has been on a certain subset of terms of a language, which we have traditionally called 'logical terms' (or 'logical constants', if focus has been on formal languages). If we wish, we can continue to call the arguments that are guaranteed to preserve truth through these terms logically valid. This does not make consequences which arise from the meaning of other terms less interesting. We are dealing with a difference in focus when studying different kinds of consequence, not a difference in kind between the various types of consequences. ${ }^{63}$

\footnotetext{
63 Though perhaps the consequences do differ in degree in some sense, as they do differ in the amount of terms whose semantic is taken into account. This sets analytic consequences apart from other types in that they could not fail to preserve truth in virtue of the meaning of all terms. At the other end of the spectrum we have the consequences that preserve truth in virtue of the meaning of no terms.
} 
The principal insight of Etchemendy's can be summed up in three simple lines. Some natural language arguments are good arguments in that in virtue of the meanings of the terms they contain it could not happen that such an argument did not preserve truth. When this happens the conclusion follows from the premises, or we say that the conclusion is a consequence of the premises. These consequences are the primary study of logic, at least from a philosophical perspective, as they represent correct arguments. However, we sometimes are interested in arguments that are truth preserving in terms of the meanings of a given class of terms. Traditionally, the enterprise of logic falls into this category, as focus has been, as a matter of fact, on a rather narrow class of terms of a given language. There is nothing wrong with this, but we should remember that the decision to focus on a single class of terms does not result through a principled distinction between the terms which is ultimately due to special properties that these terms may have. All terms have a meaning and all terms are capable of securing the truth of certain statements through that meaning. Having a principled focus on a special set of terms illegitimately narrows the scope of logic; the study of arguments that guarantee the truth of their conclusions upon the truth of their premises. Here is how Barwise \& Etchemendy [1989] describe the phenomenon of logical consequence:

One of the driving forces behind model-theoretic semantics has been the desire for an account of entailment. Just what is it for one statement to "follow logically" from another? Intuitively a sentence is logically true if it is true in virtue of the semantic properties of the language, independent of how other facts happen to fall out. Similarly one sentence logically entails another just in case the second is true if the first is - again, simply in virtue

These are the consequences where one of the premises is identical to the conclusion. It may sound weird to say that these could not fail to preserve truth, though this is due to the meaning of none of the terms, but this does seem to be what happens. What guarantees the truth preservation is the identity of the given terms, not their meaning. 
of the semantic properties of the language. [1989: 224]

But this is just a restatement of (sem) above. This notion of logical truth and logical consequence comes about when each way the facts could have been is represented by a model, and the semantics of all terms are taken into account in defining truth in a model. We thus have reasons to accept that something like Shapiro's blended notion of consequence is accepted by Etchemendy as that which the model theory aims to capture, or model to use Shapiro's term. However, for Etchemendy the decision to treat some terms as logical and others as non-logical is a pragmatic choice and the dividing line between the two is equally pragmatic. ${ }^{64}$

But, even though the choice is pragmatic, it may be necessary, though necessary for modeling the resulting phenomenon, not for understanding the content of (sem). The thought behind this is that a model theory which respects the semantic contribution of all terms throughout all models is intractable because of its sheer complexity. It might therefore be a pragmatic choice which must necessarily be made, though pragmatic nevertheless. We should note, however, that if such a choice is necessary it does not show that the important notion is not that given in (sem). Rather, it would show only that model theory cannot be an adequate model of this phenomenon, perhaps because the phenomenon is too complex to be captured in rigorous mathematical terms.

\subsubsection{What Is Part of The Semantics?}

In the previous section we saw evidence to the effect that Etchemendy endorses an account of a logical truth as a statement which is true in virtue of the semantic properties of the language, independent of of how the facts of the world happen to be.

64 Of course, Etchemendy also does not call the terms 'logical' and 'non-logical', respectively. What we mean by the above is just that the formal counterpart of a term treated as a logical term receives the same interpretation in all models, whereas the interpretation of the formal counterpart of a term treated as a non-logical term is allowed to vary from model to model. 
Analogously, an argument is logically valid if it preserves truth in all possible worlds in virtue of the semantic properties of the language. This gives us necessary truth or necessary truth preservation in virtue of the semantic properties of the language. ${ }^{65}$

This, as we saw, is exactly what the model theory is meant to be a model of: each model represents a way that the facts of the world could have been which is relevant to the semantics of the language, and the semantic properties are modeled by the

${ }^{65}$ Etchemendy uses the phrase 'logically possible way the world could have been', where the emphasis is on 'logically'. This may lead us to suspect that Etchemendy has something different from a metaphysically possible way the world could have been in mind. To equate Etchemendy's views with Shapiro's, as we have suggested above, we would need Etchemendy to endorse that logical consequence is truth preservation in all metaphysically possible worlds in virtue of the semantics of the involved terms. Now, whether or not Etchemendy does endorse such a view, I take it that it has been shown above that such a view is at least compatible with Etchemendy's comments and that, quite independent of Etchemendy's views, the notion is interesting in its own right, and therefore worth studying, as it can account for many of our intuitions concerning logical consequence.

That said, I do believe that the most natural reading of Etchemendy is as endorsing this particular view. A 'logically possible way the world could have been' is a metaphysically possible way that the world could have been. The 'logically' serves only to indicate that each model represent a possible way the world could have been, in this absolute sense, which is relevant to the truth or falsity of the sentences of the language: a clause which is explicitly added in many of Etchemendy's formulations. As such, what is being stressed here is that the possible ways the world might have been must carry semantic relevance. If our language cannot express that there is a difference between two different metaphysical possibilities, then they will be the same logically possible way the world could have been. This, of course, is not to say that logically possible ways the world could have been become an ontological category in themselves. Logically possible ways the world could have been are ways the world could have been which are relevant to the semantics of the language in question. What is being stressed by the use of 'logical' here is that logical consequence - the relation of interest whether we call it 'logical consequence' or not - is a semantic phenomenon. Consider, for illustration, a propositional language where the atomic sentences are treated categorematically, and consider a model which interprets the atomic sentences so that the truth of none of them depends on the color of The White House. For each possible color of The White House there will therefore be different metaphysical possibility which for the purposes of this interpretation are the same logically possible way the world could have been. What is logically possible is therefore relative to the language in question, but that does not create a different ontological category.

One might naturally worry that these last comments suggest that there are more metaphysically possible worlds than there are logically possible worlds. But this is not the case. A logically possible way the world could have been just is a metaphysically possible way the world could have been along with an interpretation of the non-logical terms. When we claim that for the purposes of this interpretation of the language, the different metaphysically possible worlds are the same logically possible way the world could have been, we mean so only relative to individuating a logically possible world as those facts that are relevant to the truth or falsity of the sentences of the language. And under this particular interpretation, the color of The White House is not so relevant. However, since there are clearly many interpretations of the language for which the color of The White House does matter, there will, on this way of individuating logically possible ways the world could have been, be many logically possible ways the world could have been for which this particular metaphysically possible world is not the same logically possible way the world could have been. 
truth clauses given independently of any one model. This represents that the semantic properties of the language are fixed and are the same for all representations of the world: it is consequently in virtue of these properties that a valid argument preserves truth no matter how the world happens to be, i.e., preserves truth necessarily. Ideally, a model-theoretic semantics encodes the semantic properties of all terms by producing an adequate truth clause for all terms, but Etchemendy allows that we focus only on the logic, so to speak, of a few select terms, whereas the precise semantic functioning of the remaining terms is ignored in lieu of giving the semantics of these terms a uniform treatment. One might even think that a treatment of at least some terms as categorematic is necessary for the model-theoretic machinery to be employed. Perhaps it is just too much to expect that we can have a mathematical model of the semantics of all terms of a language, assuming, that is, that the language is sufficiently similar to natural language in its expressive power. We will not deal with these issues here, but there can be no doubt that a model theory capable of representing all possible configurations of the world as well as giving the semantic functioning of all terms of natural language would have to be extremely complex.

One thing we will enquire about, albeit briefly, is how exactly we are to understand 'semantic properties' or the 'semantic functioning' of terms. Here is a case to motivate the issue. Suppose we understand 'semantic properties' simply as meaning. Suppose furthermore that we accept the Millian account of names according to which the meaning of a name just is its referent. ${ }^{66}$ If each model is to respect the meaning of all terms and represent a way that the world could possibly have been, this entails that 'Hesperus is Phosphorus' is a logical truth, in the sense in which logical truth is truth in virtue of meaning, independently of how the facts are. Let ' $a=b$ ' be the formal translation of the sentence in question. Since each model takes into account the

\footnotetext{
${ }^{66}$ For a discussion of this view in relation to competing views, see Kripke [1980].
} 
meaning of the terms ' $a$ ' and ' $b$ ' ('Hesperus' and 'Phosphorus', respectively), and since their meaning is their referent, their referent should be, in each model, the object that represents Venus. A model can make this false only if it can represent Venus not to be Venus. But, of course, there is no such possibility to represent, hence, 'Hesperus is Phosphorus' passes the test of being logically true. This, however, does not square well with the intuition of some theorists; especially those who accept Formality as a condition on logical consequence and logical truth. If 'Hesperus' and 'Phosphorus' are non-logical terms, then 'Hesperus is Phosphorus' is not a logical truth, since not all sentences of this form are logical truths; i.e., 'Mars is Venus'. Of course, from Etchemendy's perspective the problem is not that we should accommodate Formality, since this is clearly false as it relies on a distinction between logical and non-logical terms. However, one might still feel that 'Hesperus is Phosphorus' should not be a logical truth.

It is clear that Shapiro's account can accommodate this intuition because it relies on a distinction between logical and non-logical terms, the former excluding names. ${ }^{67}$ Hence, what matters is not that given the meaning of the names 'Hesperus' and 'Phosphorus' there is a model representing the two as referring to distinct objects. Rather, this is not a logical truth because there is a possible world (the actual world being a prime candidate) in which we can interpret the names 'Hesperus' and 'Phosphorus' to apply to distinct objects, say, Mars and Venus. Hence, there is a world and an interpretation of ' $a$ ' and ' $b$ ' making ' $a=b$ ' false.

It is perhaps physically or metaphysically or analytically impossible for Joe to be lighter than Bill and Bill to fail to be heavier than Joe, but it is logically possible for this to happen. (Shapiro [2005])

On Shapiro's account, a logical possibility just is a genuine possibility but with a ${ }^{67}$ We will look at the details of this in the next section. 
possible non-actual interpretation of the non-logical terms. This is what allows us to accommodate the intuition behind Formality. We can explain this intuition by excluding 'heavier than' and 'lighter than' as logical terms. But, since Etchemendy denies that there is any real distinction to be had between logical and non-logical terms, Etchemendy cannot do so on his account. What is properly logically possible is determined by the semantics of all terms including 'heavier than' and 'lighter than'. And if we assume that their meaning just is the relation which they pick out, we cannot explain the intuition that it is logically possible for Joe to be lighter than Bill and Bill to fail to be heavier than Joe. Of course, at this point we should, from Etchemendy's perspective, simply deny the intuition. There is no real situation, whether actual or counterfactual, in which Joe is lighter than Bill and Bill fails to be heavier than Joe, and this is excluded by the semantic properties of 'lighter than' and 'heavier than'. No situation is such, and the purported "logical possibility" rests on a change in meaning of the terms.

I have no doubt that Etchemendy would be happy to accept that it is not logically possible for Joe to be lighter than Bill and Bill to fail to be heavier than Joe when logical possibility is understood as not necessarily false in virtue of the meanings of the terms. This is just the way in which Etchemendy choose to use the technical term 'logical possibility'. Of course, when we take into account the meaning of 'lighter than' and 'heavier than' we see that the statement expressed by 'Joe is lighter than Bill and Bill fails to be heavier than Joe' is in fact necessarily false. The sense in which it is logically possible for Joe to be lighter than Bill and Bill to fail to be heavier than Joe is a derived sense: if we pay attention only to the semantics of the terms traditionally treated as logical constants and treat the remaining terms categorematically, we see that is is not necessarily false that Joe is lighter than Bill and Bill fails to be heavier than Joe in virtue of the semantic properties of only the 
former terms. We can thus account for the intuition behind Formality as well, though only from a pragmatic perspective. A version of Formality will hold relative to the terms treated categorematically. But, of course, since the proper consequence relation emerges when no terms are treated categorematically, we cannot straightforwardly accommodate the intuition that 'Hesperus is Phosphorus' is not a logical truth; at least insofar as we accept that the meaning of both names just is Venus.

If one wishes to deny that 'Hesperus is Phosphorus' is a logical truth from Etchemendy's perspective, one could perhaps maintain that the relevant semantic properties of names are not their referent, but rather some general facts about how they contribute a referent to an occurring expression. ${ }^{68}$ But if we push too far in this direction for names, it seems we are unable to explain the difference between paying attention to the semantic properties of a name and treating names categorematically. If the semantic properties of names which every model must respect just is that a name contributes with a referent to an occurring token of that name, then is this not just treating names categorematically? Yet, according to Etchemendy, there is an important distinction between the two.

Similarly, if we want to explain why 'All bachelors are unmarried' is not a logical truth - an intuition which, no doubt, a lot of people share - we might point out that the relevant semantic properties for predicates, which each model should depict, is just that a predicate contributes with an extension, and nothing more. But, again, we lose the ability to explain the difference between paying attention to a term's semantics and treating a term categorematically. And what is more, we seem to be smuggling the notion of a logical constant back into the framework, though this time it is a distinction which is of utmost importance and not just a pragmatic distinction. A logical constant

${ }^{68}$ Let me stress, though, that I am speaking about the possibility of someone accepting Etchemendy's insights and wanting to accommodate Formality in a principled manner. Etchemendy himself, of course, does not believe that logic is formal in the requisite sense (Etchemendy [1983]), and satisfying the tenet of Formality can only ever be regarded as an accidental upshot of the modeling techniques. 
is one such that its full meaning, on some way of making sense of this, is taken into account. ${ }^{69}$

There is evidence to the effect that epistemic considerations should settle the matter. When explaining the validity of modus ponens, as we saw, Etchemendy stresses that we recognize its validity by knowing the semantics of the expression 'if...then...' and knowing that no matter how the world determines the truth values of the constituent parts, the result will preserve truth. In [1990] we see Etchemendy stress the $a$ priori nature of logical consequence.

For example, some would claim, not implausibly, that it is only due to the a priori relation between the premises and conclusion of a valid argument that we judge the latter to follow necessarily from the former, and hence that we judge the argument valid. On this view, a necessary consequence

${ }^{69}$ Sagi [2013: 111ff] proposes a framework of semantic constraints. A semantic constraint is a condition put on the admissible models over which we define logical consequence. It is given by a sentence in the metalanguage which states how a given term can be interpreted by a model. We therefore consider a fully interpreted formal language. Some examples of semantic constraints are:

- $I($ bachelor $) \subseteq I$ (unmarried $)$

- $I($ even $) \cap I($ odd $)=\emptyset$

- $I($ wasBought $)=I($ wasSold $)$.

Only models whose interpretation function, $I$, meets these conditions are to be allowed in the class of models over which we define logical consequence. Semantic constraints thus serve to constrain the available interpretations of a given term.

From our present perspective, the terms that are restricted by semantic constraints without having their meaning fully determined in each model are simply being treated as categorematic terms. The notion of a categorematic term was introduced to allow us to abstract away from the specific content of a given term, but the level of abstraction need not necessarily be an all or nothing affair. That is, we need not decide that all terms of a given semantic category have to be treated the same, whether that be categorematically or not. The identity relation is often excluded from the categorematically treated terms designating other relations, so such an "all or nothing" division is not even present in standard logic. When imposing a constraint such as $I($ even $) \cap I($ odd $)=\emptyset$ we are still treating the terms even and odd categorematically, but instead of seeing these as simply delivering an extension, we are now constraining each model to further details concerning the semantics of the terms in question; namely that their intersection is always empty: in virtue of the very meaning of 'being even' and 'being odd' nothing can be both. 
that could not be recognized as such a priori would never qualify as a logical consequence. And this certainly seems right. [1990: 89]

This suggests that the part of the semantics for the terms which is kept constant across models is the part that can be known a priori. A logical consequence comes about when it happens that due to the semantics of the terms a given argument cannot have true premises and a false conclusion, and we are in a position to know this a priori. This seems to leave us with a way to explain away an example such as 'Hesperus is Phosphorus', since it is commonly agreed, following Kripke [1980], that it cannot be known a priori that Hesperus is Phosphorus. Whether or not this would rule out 'All bachelors are unmarried' as a logical truth would depend on whether it can be known a priori that all bachelors are unmarried, but we may note, that from the perspective of Etchemendy, we should accept that 'All bachelors are unmarried' is a logical truth. It is a prime example of an analytic truth, and hence it is true in virtue of meaning of the involved terms. What matters for our present purpose is not how we answer these questions, but simply this: there may be intricate questions relating to how to determine the proper semantics of a natural language term, and what aspect of this is to be kept constant across models when modeling the phenomenon of logical consequence. From the perspective of model theory, though, it should be clear that the answers are to be obtained elsewhere. Whatever the answers are, we presuppose that the answers are the right ones when setting up our model theory and when judging it adequate or inadequate. This is just Etchemendy's point again, that the model theoretic perspective, as he sees it, does not analyze the notion of logical consequence, but presupposes it in the sense spelled out above. 


\subsection{Logical Constants and The Isomorphism Property}

As we saw, Shapiro's blended notion of consequence is the following:

$\Phi$ is a logical consequence of $\Gamma$ if $\Phi$ holds in all possibilities under every

interpretation of the nonlogical terminology in which $\Gamma$ holds. (Shapiro

[1998: 148])

But since this blended notion explicitly mentions non-logical terminology, there is a side of the story which is yet to be told: how is the division between non-logical and logical terminology to be decided?

According to Shapiro, any model theory worthy of its name has the isomorphism property [1998: 151-152]. The isomorphism property is a property of a model theory in relation to a language. To define it, we need the notion of an isomorphism. Let $L$ be the language under consideration.

(ISO) Two models, $M, N$, are isomorphic, $M \simeq N$, if and only if there is a bijective function, $f: D_{M} \mapsto D_{N}$, s.t.

i. For all individual constants, $a, I_{M}(a)=o$ if and only if $I_{N}(a)=f(o)$.

ii. For all n-ary predicates $P$ of $L,\left\langle o_{1}, \ldots, o_{n}\right\rangle \in I_{M}(P)$ if and only if $\left\langle f\left(o_{1}\right), \ldots, f\left(o_{n}\right)\right\rangle \in$ $I_{N}(P) \cdot{ }^{70}$

Two models are isomorphic if they exhibit the same structure when it comes to the objects, sets, and tuples that serve as the interpretation of various terms. An immediate upshot of this, of course, is that two models can be isomorphic only if their underlying domains are of the same cardinality. ${ }^{71}$

\footnotetext{
70 This is assuming that the language contains only individual constants and n-ary predicates. The conditions on isomorphisms extend in the obvious way to languages containing functional symbols. See Mendelson [1997: 111].

71 This is clearly related to Sher's views of model theory as given in [1991, 1996, 2008]. As we shall later see, one can prove that a given model theory satisfies the isomorphism property if and only if the
} 
The isomorphism property is the following:

(IP) Suppose the models $M$ and $N$ are isomorphic, $M \simeq N$, and let $g$ and $g^{\prime}$ be assignment functions for $M$ and $N$ respectively, such that for all variables $x$, $g(x)=o$ if and only if $g^{\prime}(x)=f(o)$. Then $M$ and $N$ are said to have the isomorphism property if and only if, for all $\alpha$ of $L, M \Vdash_{g} \alpha$ if and only if $N \Vdash_{g^{\prime}} \alpha .^{72}$

Focusing on the closed sentences of the language, the isomorphism property thus states that if two models are isomorphic, then they will make the same sentences true. Now, this may seem confusing to some readers simply because there is a tendency to think of the isomorphism property as being part of the definition of an isomorphism. ${ }^{73}$ It is often said that two isomorphic models will be elementarily equivalent, where two models are elementarily equivalent if they make the same sentences true. This, of course, is just a restatement of the isomorphism property. We can, however, define model theories for which the isomorphism property does not hold, though it will be true when we restrict attention to the standard choice of logical constants for a language - the reason follows below. Consider our predicate $B$ from chapter 1 , which we introduced as a logical constant with the following truth clause for any term $t$ and model $M$ :

(tB) $M \Vdash_{v} B t$ if and only if $\operatorname{den}(t)_{M, v}$ is blue.

logical constants of the formal language satisfy Sher's criterion of being invariant under isomorphisms (Sagi [2011: 203ff]). See section 2.2.1.

${ }^{72}$ As noted by Sagi [2011: 202, n.1], Shapiro's original definition does not mention assignment functions, but it is nevertheless natural to extend the notion to include formulae with free variables. In this respect, we need to explicitly mention the assignment function relative to which these formula are evaluated.

${ }^{73}$ As we go on to explain, it is not. The notion of an isomorphism is given relative to the interpretations of the non-logical constants, so for certain choices of logical constants two models can be isomorphic vis-á-vis their non-logical constants, as the definition requires, but not exhibit the isomorphism property. Were we to further demand that the logical constants also satisfy some criteria of being isomorphic, the isomorphism property would be part of the definition of an isomorphism, so construed. This is just the point, as we will look at below, that satisfying the isomorphism property is equivalent to the logical constants being invariant under isomorphisms (Sagi [2011: 203ff]). 
If this predicate is added to the language, a given model theory will not satisfy the isomorphism property - insofar, of course, as we are allowed at least one blue and one non-blue object from which to construct our models.

So far we have only seen Shapiro claim that any model theory worthy of its name should possess the isomorphism property. This claim does not go against the thought that logical consequence, properly understood, just is necessary truth preservation in virtue of the meaning of all terms, but only goes to show that model theory, understood as defined by Shapiro, might very well be unable to capture this notion. This will happen if the "model theory" which results from taking into account the semantics of all terms of the language does not possess the isomorphism property.

Shapiro says that the isomorphism property is a manifestation of the intuition that the notion of logical truth and logical consequence is formal and any model theory worthy of its name should possess the isomorphism property ([1998: 151-152]). One could interpret this as Shapiro endorsing the view that logical consequence, whatever it is, is a formal notion, and therefore a notion for which we need a distinction between logical and non-logical terms in order to define what form is. It should be kept in mind though, that Shapiro admits that there are many ways of making the intuitions surrounding logical consequence sharp, and the notion captured by model-theoretic means is just one such. We should therefore interpret Shapiro to make the more modest claim that a sharpened notion of the intuitions surrounding logical consequence must somehow be formal (in the sense spelled out by the isomorphism property) in order for a model theory to be able to adequately capture it. In fact, these considerations are strengthened further by the fact that we want to model a relation which has a modal profile, but at the same time we would, everything else being equal, like to do as little metaphysics as possible.

In general, even though we are trying to maintain some modality as part 
of logical consequence, it is wise to avoid such metaphysical nightmares as 'transworld identity' in developing logical theory. The idea is to keep modality, but make it tractable. After all, logic has been more successful than metaphysics, and we do well to not hold logic hostage to metaphysical disputes if we do not have to. [1998: 149]

Shapiro is pointing out that though we would like to secure that our mathematical model captures a notion which does in fact entail truth preservation in all possible worlds, we would like to do so while having to decide on the minimal number of metaphysical questions.

Suppose that a given sentence $\Phi$ is false in a model $M$ whose domain is $d$. Given any set $d^{\prime}$ with the same size as $d$, there is a model $M^{\prime}$ isomorphic to $M$ whose domain is $d^{\prime} . M^{\prime}$ is obtained by interpreting the nonlogical items of $\Phi$ in $d^{\prime}$. By the isomorphism property, $\Phi$ is false in $M^{\prime}$. Also, let $N$ and $N^{\prime}$ be models whose domains have the same cardinality. Then $\Phi$ is satisfied by $N$ under every interpretation of the nonlogical terminology if and only if $\Phi$ is satisfied by $N^{\prime}$ under every interpretation of its nonlogical terminology. In short, the only thing that can prevent a given formula from being a logical truth, or the only thing that can prevent a given formula from being a consequence of a set of formulas, is a model of a certain size.

These considerations might provide comfort to those who are sceptical of modality. If the isomorphism property holds, then in evaluating sentences and arguments, the only 'possibility' we need to 'vary' is the size of the universe. If enough sizes are represented in the universe of models, then the modal nature of logical consequence will be registered. It is a pleasing situation. [1998: 152] 
According to Shapiro, the only modal considerations which we need to engage in are considerations having to do with possible sizes of the universe. This is enough to register the modal character of logical consequence. The idea seems to be something like the same intuition which we saw expressed by McGee in chapter 1. McGee [1992], pointing out that sets exist necessarily, makes the following point:

Suppose there is a possible world $w$ in which there is a model $\mathbf{M}$ in which $\varnothing$ is false. Since the [principle] is true in every world, there exists in $w$ a model $\mathbf{L}$, isomorphic to $\mathbf{M}$, which is a pure set. Being an object of pure mathematics, L exists in every world, and it is, in every world, a model in which $\varnothing$ is false. In particular, $\mathbf{L}$ bears witness to the fact that, in the actual world, there is a model in which $\varnothing$ is false. [1992: 276]

The principle referred to is the principle stating that for any model, $M$, there exists a model, $N$, such that $M \simeq N$ and $D_{N}$ consists of pure sets. This principle certainly seems true, and it seems necessarily true too. But as pointed out in the first chapter, the reasoning also tacitly relies on the model theory satisfying the isomorphism property. We pointed this out by considering the weird logical constant $B$, a predicate true of a term $t$ if and only if the denotation of $t$ in a given model is blue. But under the assumption that the model theory satisfies the isomorphism property, $B$ is being excluded from being treated as a logical constant. I take it that the intuition which is being pressed by McGee, to the extent that he tacitly relies on the isomorphism property being satisfied, is something like the following argument:

Suppose that the argument from the natural languages sentences $K$ to $X$ is not necessarily truth preserving. Then there is a possible world, $w$, such that $K$ consists of true sentences but $X$ is false. ${ }^{74}$ Consider the formal counterparts, $\Gamma$ and $\Phi$, of $K$ and $X$,

\footnotetext{
${ }^{74}$ Strictly speaking $w$ could be the relevant part of a possible world, pace the discussion of restrictions due to quantifiers.
} 
respectively, and consider the intended model of $\Gamma$ and $\Phi$ in $w$ relative to $K$ and $X$. By this we mean the model which assigns the objects to the non-logical constants of $\Gamma$ and $\Phi$ which correspond to the objects that are the denotations of the corresponding non-logical terms in $K$ and $X .{ }^{75}$ Call this model $M$. Then $M \Vdash_{g} \gamma$, for all $\gamma \in \Gamma$, and $M \Vdash \forall_{g} \Phi$. But by the isomorphism property there is an isomorphic model $N$, consisting of pure sets, such that for the "isomorphic" assignment function $g^{\prime},{ }^{76} N \Vdash_{g^{\prime}} \gamma$, for all $\gamma \in \Gamma$, and $N \forall_{g^{\prime}} \Phi$. But, since $N$ consists of only pure sets it exists and is the countermodel to the inference from $\Gamma$ to $\Phi$. Since $\Gamma$ and $\Phi$ were formal renderings of $K$ and $X$, we see that the lack of a countermodel entails the lack of a possible world in which $K$ consists of true sentences and $X$ is false. This, of course, secures the modal profile of the logically valid arguments: if $\Phi$ is true in all models where all of $\Gamma$ is true, then for any corresponding interpretation of these, it is necessary that if the premises are true, then the conclusion is true.

As should be clear, a "proof" like this is not as straightforward as the quote from McGee might lead us to believe. It relies, for example, on the fact that for any interpretation of a non-logical term in a possible world, the object that is the denotation under that interpretation exists in that world. This is needed to secure the intended model and consequently the model isomorphic to the intended model. While this assumption may strike most as relatively innocent, at least for extensional languages, it is unclear that it is true for intensional languages. Of course, the above argument is meant to be an argument for extensional model theories, but one should nevertheless make the

\footnotetext{
${ }^{75}$ At this point we are relying on $K$ and $X$ not making any claims which require for their truth (or falsity) that the domain of discourse is a proper class, not a set. An argument from Kreisel [1967], however, shows that we need not worry about this if the language has a (nice) deductive system which is complete for the semantics. Being true on all interpretations on sets coincides with being true on all interpretations on classes. (Sets are classes, but not all classes are sets). We will look at this argument in the next section.

${ }^{76}$ We have only defined isomorphisms for models. What we mean by the "isomorphic" assignment function, $g^{\prime}$, is the function defined by $g^{\prime}(x)=f(o)$ if and only if $g(x)=o$, for all variables $x$; where $f$ is the isomorphism between $M$ and $N$. Clearly this assignment function is well-defined for the model $N$.
} 
assumptions on which it rests clear.

We also need to secure that the interpretations which are counterexamples to a given argument in a given possibility do not rely on the domain of discourse being a proper class. If we cannot be sure of this, then we cannot be sure that any countermodel exists, since models have sets as their domains. This situation can be remedied when the model theory has a nice deductive system which is complete for the semantics. ${ }^{77}$ This therefore restricts the argument to languages that have such a proof system, such as first-order and propositional languages.

\subsubsection{The Equivalence of Two Criteria}

From the perspective of the interpretational approach to model-theoretic semantics- an approach which endorses (INT) and (MRA) - the question of what terms are logical constants is essential. Without such a distinction the definition of a logically valid argument cannot be applied, since we do not know which terms to vary the interpretation of. But, as we saw, there just is no choice of logical constants which can secure a modal profile for logically valid arguments; under the given definition logical necessity is undefined over non-actuality. As Etchemendy points out, what is really being asked from this perspective is this: when does the definition get the extension right (Etchemendy [2008: 282])? There is nothing in the definition relying on (INT) and (MRA) that can secure the modal profile, and it comes about purely through happenstance: the model theory which delivers the extension happens to represent all possibilities and the valid arguments will therefore be necessarily truth-preserving.

On the representational approach, the logical/non-logical distinction does not seem to play a very important role. This is especially true for Etchemendy as we saw: the

\footnotetext{
77 We will define the notion of a nice deductive system in section 2.3. As will become clear then, one might want to put the restriction of being nice on a deductive system proper. For this reason we do not make a great deal out of emphasizing that the deductive system should be nice, since almost all such systems (that are seriously proposed) will have this feature anyway.
} 
interesting concept is necessary truth preservation in virtue of the semantics of the terms, where, ideally, the semantics of all terms should be taken into account. The decision to treat some terms as logical constants is purely pragmatic, though it may be, as we suggested, necessary as well.

Shapiro, as we saw, imposes the isomorphism property as a criterion on any model theory. As it turns out, this is equivalent to accepting only as logical constants operators and functors that are invariant under isomorphisms. This criteria is an extension of the ideas found in Tarski [1986], but in its present form it is due to Sher [1991, 1996, 2008].

I will not rehearse the details of Sher's criterion of Invariance-under-Isomorphisms, but the central idea is just that a term is a logical term if and only if it refers to an operation which is invariant under isomorphic structures. ${ }^{78}$ As Sagi [2011: 203ff] proves, these two criteria are in fact equivalent when considering standard first-order languages with an accompanying standard model theory. The sense in which they are equivalent is that a first-order language with accompanying model theory will have the isomorphism property if and only if the constants that are designated as logical satisfy Sher's criterion.

It is not the point of the present thesis to assess the merits and demerits of Sher's theory of logical consequence. ${ }^{79}$ As we saw, Sher attempts to provide a principled

\footnotetext{
${ }^{78} \mathrm{~A}$ logical term is part of natural language whereas a logical constant is part of a formal language. Sher's definitions are given in set-theoretic terms, and so apply first and foremost to formal operations. According to Sher, logical terms are formal in the sense of denoting properties and relations that are intuitively structural or mathematical (Sher [1996: 674]). By intuitively structural or mathematical Sher means that they satisfy her criterion, so we can see how natural language terms are defined as logical by denoting formal operations that are invariant under isomorphisms. Now, apart form truth functional operators, Sher defines a logical constant (as part of the formal language) to be an $n$-place predicate or a functor of level 1 or 2, satisfying certain intuitive restrictions (for example, for predicates, their extension should be a subset of the domain). Briefly, such a constant is logical if for any two isomorphic structures $M$ and $N$, the extension of the constants as drawn from $D_{N}$ include all and only the images of objects, under $f$, that are the extensions of the constants as drawn from $D_{M}$; where $f$ is the isomorphism from $M$ into $N$. The exact details can be found in Sher [1991: 54-55].

${ }^{79}$ For such an attempt I refer the reader to the vast literature on establishing a theory of logical consequence through a demarcation of the logical and non-logical terms.
} 
distinction between a term being logical and a term being non-logical, extending ideas from Tarski [1986]. But what it means for Sher for a term to be logical is just that it is structural, or mathematical, in some sense. The models, on Sher's account represent formally possible structures. The idea is that the meaning of the logical constants give rise to the logically valid arguments through their formal and structural properties, when the models represent formally possible structures.

On my conception, formal possibility is reduced to mathematical existence and formal necessity to mathematical generality: "It is formally possible that $\Phi "$ to "There exists at least one mathematical (set theoretical) structure $\mathcal{S}$ such that $\Phi$ holds in $\mathcal{S}$ " and "It is formally necessary that $\Phi$ " to "For all mathematical (set theoretical) structures $\mathcal{S}, \Phi$ holds in $\mathcal{S}$. (Sher [1996: 682])

We may note that the important concept for Sher is that of a formally possible structure, which is different from a possible world. ${ }^{80}$ But since a formally possible structure is defined in terms of mathematical structures, one might think that we have a genuine reduction of logical consequence to something both well-understood (to a certain extent) and modally innocuous. But this cannot quite be the case, since there is a difference between being invariant under actual structures and invariant under possible structures, and Sher intends her notion to apply to the latter. This point can be brought out using an objection from Gómez-Torrente [2002: 18ff] to Sher's theory of logical constants. Consider the natural language predicate 'Unicorn'. The extension of this predicate in a model is presumably the set of unicorns. But, since there are no unicorns, it will have an empty extension in all models, and consequently it will satisfy Sher's criterion for being a logical constant. Sher replies to Gómez-Torrente's

${ }^{80}$ Sagi [2013: 32ff] contains a thorough discussion of Sher's view in comparison with Shapiro's. Sagi argues that the latter is to be preferred because Sher introduces a new primitive notion of a formally possible structure, which we can avoid on Shapiro's view. 
point by showing that these terms are not logical on her account. "[F]or the given language; in particular, any set whatsoever, whether of existent or fictional objects, is the universe of some model" (Sher [2003: 193]). This, of course, excludes 'Unicorn' as a logical constant, since the extension varies between models that contain no unicorns and models that contain unicorns. But now it seems the models which we are using to define logical constants can contain non-existent objects.

This does cast some doubt on whether the criteria set forth by Shapiro (i.e., the isomorphism property) is truly the same as the criteria put forth by Sher (i.e., invariance under isomorphisms) for certain languages. There can be no doubt that the proof given in Sagi [2011] is correct, but it does rely on the fact that the entities making up the models and deciding whether a term is invariant under isomorphisms are either all existing objects or non-existent objects. If the model theory is defined on models consisting of only existent objects, then it can possess the isomorphism property without the logical constants satisfying Sher's criterion. ${ }^{81}$ 'Unicorn' is a case in point. A model theory for which the models consist of only existent objects will have the isomorphism property if it possesses the property in the first place and the following logical predicate is added: ${ }^{2}$

(tU) For any term $t$, any model $M$, and assignment function $g$; $M \Vdash_{g} U t$ if and only if $\operatorname{den}(t)_{M, g}$ is a unicorn.

\footnotetext{
${ }^{81}$ Obviously, the converse is the case too: if the domains over which we decide whether a term satisfies Sher's criterion consist of only existing objects, whereas the model theory satisfying the isomorphism property can include non-existent objects in the domains, the two criteria will come apart. But Sher explicitly claims that she means her criterion to apply when all possible domains are considered [2003: 93].

${ }^{82}$ Assuming, that is, that unicorns do not exist. This is actually a little trickier than we make it out to be in the text. One common approach dictates that while unicorns are not actual extended entities in our world, they nevertheless exist, though as abstract objects. If this is the case, then they should be able to figure as elements in the domain of a model and the above example is not a counterexample. One might consider using a predicate such as 'being the seventh son of Kripke', but on many approaches even this object exists, though of course, it is abstract too. (Kripke's own theory of fictional objects seems to be one such. See Kripke [2013: 55ff].)
} 
But if the domains for which a term needs to be invariant under isomorphisms can contain unicorns, then $U$ will not be a logical constant according to Sher's criterion. Sher denies that this satisfies her criterion for being a logical constant for just this reason, but it is clear that under the assumption that there are not any unicorns to play the roles of members of the domains, any model theory having the isomorphism property will continue to have it after the addition of the predicate $U$. Now, it might be said that this is only apparent since even though there are no actual physical unicorns, they nevertheless exist as abstract objects, and can therefore play the roles of the members of the domains. This, of course, solves the issue pertaining to the predicate $U$. Presumably, though, the isomorphism property was meant to secure that the exact nature of the objects from which our models are built cannot affect the output of the semantics. Yet, if the models contain no unicorns, $\neg U a$ will be a logical truth. It seems the isomorphism property is not quite strong enough to rule out that the output of the semantics is influenced by the exact nature of the objects of the domains. None of this, of course, is meant to be a criticism of Shapiro who holds that the isomorphism property is necessary for any model theory worthy of the name, not necessarily sufficient. We are merely pointing out that whether a given model theory possesses this property will also depend on what objects are available to construct the domains out of in the first place. If unicorns are available, then $U$ is ruled out. If not, not.

From Sher's perspective there is a principled distinction between being a logical and being a non-logical term. From the perspective of Etchemendy, a perspective we have adopted here, no such division exists. Focusing on only the semantics of some terms is a pragmatic decision, though I leave it open whether it is forced on us due to the tractability of a model theory taking into account the semantics of all terms. Picking logical constants that are invariant under isomorphisms gives rise to "wellbehaved" model theories in the sense of them possessing the isomorphism property, 
but from the present perspective the logical constants are still just that: invariant under isomorphisms.

From Sher's perspective each model represents a formally possible structure, and in relation to that it becomes important to pick the right logical constants, the ones that also reflect formal, or structural, properties. But at this point we seem to be dealing with a relabeling of the term 'logical'. Sher equates logical possibility with the existence of certain mathematical structures, and logical necessity and logical consequence consequently manifest themselves through mathematical necessity. Such a theory might very well be worth studying extensively, but from our present perspective, the inter-

esting concept would still be the concept of necessary truth preservation through the semantics of the terms; at least insofar as we are concerned with actual reasoning. That is, even were we to agree to call the notion of consequence which Sher's theory gives rise to logical consequence, there still would be another interesting concept of necessary truth preservation due to meaning, which is clearly worth studying.

\subsection{Meta-Theorems}

Etchemendy [1990: 3ff] points out that there is something odd in the naming of soundness and completeness theorems. Soundness and completeness, as we use them here, are technical terms which apply to a semantic definition of consequence and a corresponding proof-theoretic definition. Let $\Gamma \models \alpha$ denote that the semantic consequence relation holds between $\Gamma$ and $\alpha$, and let $\Gamma \vdash \alpha$ denote the corresponding proof-theoretic relation. Soundness and completeness theorems are of the following form. For all $\Gamma$ and $\alpha$ of the language.

(COM) If $\Gamma \models \alpha$, then $\Gamma \vdash \alpha$.

(SOU) If $\Gamma \vdash \alpha$, then $\Gamma \models \alpha$. 
As such, obviously, these theorems are just mathematical theorems about the relationship between two alternative characterizations of logical consequence in a formal language. However, what Etchemendy notes is that the naming of them conveys an unjustified assumption that the semantic definition is an obvious analysis of the target phenomenon.

But we think of these results as having an intuitive significance that goes beyond the mere coincidence of two alternative characterizations of the consequence relation. Specifically, we think of them as demonstrating the extensional adequacy of the deductive system in question. (Etchemendy [1990: 3])

Now, it is clear that a claim such as the one Etchemendy is making will be extremely difficult to assess. Who are the 'we' who think this? Etchemendy is presumably talking about logicians (perhaps philosophers too). The tendency is one of seeing completeness and soundness theorems as establishing the extensional correctness of $\vdash$. This is further reflected in the fact that we often predicate the properties of being sound and complete of a given deductive system, but never of the model theory.

The felt asymmetry in our original two theorems stems from our assumption that the model-theoretic definition of consequence, unlike syntactic definitions, involves a more or less direct analysis of the consequence relation, and so its extensional adequacy, its "completeness" and "soundness," is guaranteed on an intuitive or conceptual level, not by means of additional theorems. If it were not for this assumption, we would feel equal need for external evidence that the model-theoretic characterization of consequence is extensionally correct, that it applies to all valid arguments, and only valid arguments, of the language in question. [1990: 4] 
Once we realize that the model theoretic definition does not provide such a straightforward analysis - something which we have argued extensively for in the first chapter - we can reassess the significance of the completeness and soundness results. As we shall see, a completeness theorem can actually come to function as a "soundness" theorem for the model-theoretic definition, where the soundness in question is relative to the genuine consequence relation. The completeness theorem states that if something is a model-theoretic consequence, then it is also a deductive consequence. If we could somehow ascertain that deductive consequences are all genuine consequences, we could combine this into a "soundness" theorem for the model-theoretic consequence relation. That is (COM) and (DS), would entail (MS).

(DS) If $\Gamma \vdash \alpha$, then $\alpha$ is a genuine consequence of $\Gamma$.

(MS) If $\Gamma \models \alpha$, then $\alpha$ is a genuine consequence of $\Gamma$.

The first thing to note about (DS) and (MS), however, is that it is not at all obvious that their consequents make sense. First off, there is the question of 'the genuine consequence relation'. What is it, and how do we know anything about it? As we have argued extensively, there might be many ways to give content to a notion of logical consequence. The notion which will interest us is the notion of truth-preservation in all possibilities in virtue of the semantics of the terms. Allowing for treatment of some terms as categorematic terms (i.e., as non-logical terms), this is the notion of being truth preserving in all possibilities in virtue of the semantics of the logical terms. Let us refer to this as our informal notion of logical consequence, or I-consequence, for short.

However, another issue immediately presents itself, since I-consequence, as we have defined it, is a relation between natural language sentences and classes of sentences. $\models$ and $\vdash$ are relations between formal sentences and classes of sentences. Here, obvi- 
ously, is where we have to rely on some relationship between the formal language and natural language. For now we will leave this at an intuitive level, though we shall see shortly that the significance of the various meta-theorems can be called into question by investigating the exact relationship between formal and natural language.

Blanchette [2000] introduces the notation of $R(\varphi)$ for a reading of the formula $\varphi$. She further defines a reading to be a proposition. We will define it to be a statement in an attempt to remain neutral on the exact nature of the relata of the logical consequence relation (See Section 0.1).

[L]et $L$ be a familiar first-order language, of the kind found in standard logic texts. There are acceptable readings of this language which assign

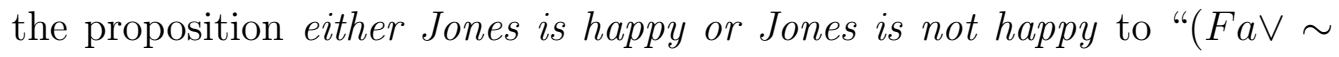
Fa)", and which assign the proposition either snow is white or snow is not white to that formula, but no acceptable reading assigns to this formula the proposition snow is white. It is a result of these restrictions on its acceptable readings that, for example, " $(F a \& \sim F a)$ " expresses a false proposition on each acceptable reading of $L$, " $(F a \vee \sim F a)$ " expresses truths on each such reading, and " $F a$ " expresses truths on some, and falsehoods on other acceptable readings of that language. Where $R$ is a reading of a language $L$ and $\varphi$ a formula of $L$, let $R(\varphi)$ be the proposition assigned by $R$ to $\varphi$. [2000: 47]

Though Blanchette does not offer a rigorous definition of acceptable reading, she gives some intuitive examples. For our purposes we will be interested in a particular reading which we will assume exists. This reading is just an interpretation given to the formal language in order to check whether a given natural language argument is valid. That is, if $\alpha$ is an adequate formalization of $X$, then $X$ is such an interpretation of $\alpha$. We will assume that such interpretations correspond to acceptable readings, and we will 
denote those readings as $R_{A} \cdot{ }^{83}$ The correct formulations of the above theorems are then:

(DSL) If $\Gamma \vdash \alpha$, then $R_{A}(\alpha)$ is an I-consequence of $R_{A}(\Gamma) .{ }^{84}$

(MSL) If $\Gamma \models \alpha$, then $R_{A}(\alpha)$ is an I-consequence of $R_{A}(\Gamma)$.

(DSL) along with the completeness theorem entails (MSL). There is a straightforward way to argue for (DSL) though it will naturally be relative to a specific language and system. Consider a deductive system for a propositional language. Assume that the axioms can all be ascertained to express necessary truths under all acceptable readings. Hence, for each axiom, $\alpha$, and an acceptable reading, $R_{A}, R_{A}(\alpha)$ is guaranteed to be a logical truth in the intuitive sense. Next, we check that the rules of inference (typically just modus ponens) have the following property: If $\alpha$ can be inferred from $\Gamma$, then for all acceptable readings $R_{A}, R_{A}(\alpha)$ is an I-consequence of $R_{A}(\Gamma)$. This secures that if $\Gamma \vdash \alpha$, then $R_{A}(\alpha)$ is an I-consequence of $R_{A}(\Gamma)$, and since both I-consequence and deductive consequence are supposed to be transitive, we will always have that if $\alpha$ can be deduced from $\Gamma$, then the reading of $\alpha, R_{A}(\alpha)$, will be an I-consequence of the reading of $\Gamma, R_{A}(\Gamma)$.

What we have here is a soundness proof of $\vdash$ relative to the informal notion of consequence which we are out to model. We might refer to a deductive system which possesses this property as nice, adapting a piece of terminology from Blanchette [2000: 55]. Combined with a completeness proof we get a soundness proof for our modeltheoretic account of consequence relative to the informal notion of consequence. Our model theory captures only formal counterparts of natural language inferences that are

\footnotetext{
${ }^{83}$ We may note that for any given formula, $\alpha$, of the formal language, there may be more than one acceptable readings of $\alpha$.

${ }^{84} R_{A}(\Gamma)$ is defined to be the set $\left\{R_{A}(\gamma) \mid \gamma \in \Gamma\right\}$.
} 
valid in the informal sense which we set out to model. ${ }^{85}$

If a given deductive system is both nice and complete relative to a semantics, we can assure ourselves that the semantics does not overgenerate; that is, does not declare arguments valid whose natural language counterparts are not informally valid. Whether or not standard characterizations of model theories and deductive proof procedures for first-order languages do in fact possess these properties is a different question, one I will not pursue. But if we accept that they do, we have thus managed to show the soundness of our model-theoretic definition relative to the informal notion of consequence. This is essentially the same result which could be proved by accepting McGee's intuition, as we saw above. We have now shown it in a two step procedure, by pointing to the soundness of the deductive system relative to the informal notion, and then inferring the soundness of the model-theoretic notion via the completeness theorem for the deductive system relative to the model theory.

The above argument shows that for certain languages, if $\Gamma$ model-theoretically entails $\alpha$, then $R_{A}(\alpha)$ is a consequence of the set $R_{A}(\Gamma)$ in Shapiro's blended sense. Since this is necessary truth preservation in terms of the semantic properties of a certain subset of the terms in $R_{A}(\alpha)$ and $R_{A}(\Gamma)$ (the logical terms), it follows that it is also necessary truth preservation in terms of the semantics of all terms in $R_{A}(\alpha)$ and $R_{A}(\Gamma)$ - it is just that the exact semantic functioning of some of them can be ignored.

\footnotetext{
${ }^{85}$ What kind of proof systems are nice? Clearly, the standard proof system for propositional languages is, but already turning to standard first-order languages things get complicated. As Blanchette [2000: 55] points out, standard first-order deductive systems count among their theorems sentences such as ' $\exists x(x=a)^{\prime}$. But it is not obvious that this theorem is necessarily true under all acceptable readings. Instead it looks like there are plenty of readings of this formula under which it is not necessary, one such coming about from interpreting ' $a$ ' as a name for Kasper: 'Kasper exists'. This, I take it, is true, but it clearly is not necessarily true. One might take this as an argument that we really ought to adopt a free logic in order to model the correct informal notion. I am tempted to take it as such. Otherwise one might attempt to exclude these readings as unacceptable. This, however, seems to be a difficult task. What fact could we point to excluding interpreting ' $a$ ' as a name for me? Surely not that I do not exist necessarily, even though, of course, this is in fact true.
} 
What this means is that we have a soundness proof of model-theoretic consequence relation (for which there is a complete and nice deductive system) relative to both the blended notion of consequence put forth by Shapiro and the informal notion of interest for Etchemendy. Model-theoretic consequence is sound for both notions under the relevant assumptions, and we consequently have a safeguard against overgeneration. Etchemendy points this out for his own informal notion (Etchemendy [1990: 150]). ${ }^{86}$

What we should note, though, is that if we view our models for our first order language in accordance with (INT) and (MRA), this holds purely by accident. It holds by nothing more than the fact that the model-theoretic semantics is also acceptable from the representational perspective. An argument for this result relying on McGee's intuition clearly sees the models as representations, and relying on the completeness proof is relying on something which is external to the definition; something which just happens to be the case. What we are really showing is that the model theory is equally acceptable from the representational perspective. It manages to represent all logically possible ways the world could have been relative to the semantics of the logical constants, and for each non-logical constant (i.e., each constant treated categorematically), a model will represent a logically possible way the world could have been for at least one natural language expression whose formal counterpart falls into that category. But the assurance of comes from the fact that the model-theoretic definition of consequence has a nice deductive consequence relation which is complete for it, something which surely is not part of the model-theoretic account of consequence seen in accordance with (INT) and (MRA).

\footnotetext{
${ }^{86}$ At this point there is a tension present in Etchemendy's work. Recall that Etchemendy denies that $\exists x, y(x \neq y)$, and so on, are logical truths. He denies this on the ground that they make a claim about the cardinality of the world: a matter on which logic should not pass judgment. However, it is clear that $\exists x(x=x)$ is a theorem of a standard first-order logic, and that this sentence equally makes a claim about the cardinality of the world; namely that it is different from zero. This is also pointed out by Griffiths [2014: n. 6]. In this case the model-theoretic definition does overgenerate. One can only speculate on the motivation behind not pointing this out. Perhaps it is simply a glitch on Etchemendy's side, or perhaps he thought it inessential in relation to the greater picture.
} 
Now, the above argument, as we have discussed, would constitute a "soundness" proof for our model-theoretic definition relative to the given informal notion. We could enquire whether we should expect a "completeness" proof to be equally obtainable. The relevant principle, which we would like to establish, is this:

(MCL) If $R_{A}(\alpha)$ is an I-consequence of $R_{A}(\Gamma)$, then $\Gamma \models \alpha$.

Now, since the model-theoretic definition of consequence for Etchemendy is an idealization, in the sense of abstracting away from the exact meaning of the terms treated categorematically, we should expect it to miss out on some valid arguments. In fact, it is not hard to show that this must be the case. Consider, for example, a prime example of statement being true in virtue of meaning: 'All bachelors are unmarried'. This is a logical truth on the informal understanding of being necessarily true in virtue of the semantics of all terms. Yet, its formal counterpart, $\forall x(B x \rightarrow N x)$, is not true in all models. Hence, we have a counterexample to (MCL) given by $R_{A}(\forall x(B x \rightarrow N x))=$ 'All bachelors are unmarried' and $R_{A}(\Gamma)=\Gamma=\emptyset$. This, of course, is as it should be. When deciding to ignore the semantics of certain terms we cannot expect to capture all arguments that are necessarily truth-preserving in virtue of the semantics of all the involved terms.

\subsubsection{Squeezing Arguments}

As we have already looked at briefly in the first chapter, squeezing arguments are a type of argument that aims to show that two concepts coincide by, so to speak, squeezing them together. Such squeezing arguments are inspired by Kreisel [1967] who uses these techniques to prove rigorous results about informal notions. Kreisel was interested in the notion of being true in all structures. 
We shall consider formulae $\alpha$ of finite order[.] [T]he predicate Val $\alpha$ to mean: $\alpha$ is intuitively valid, $V \alpha: \alpha$ is valid in all set-theoretical structures, and $D \alpha: \alpha$ is formally derivable by means of some fixed (accepted) set of formal rules. ...

The intuitive meaning of $V a l$ differs from that of $V$ in one particular: $V \alpha$ (merely) asserts that $\alpha$ is true in all structures in the cumulative hierarchy, i.e., in all sets in the precise sense of set above, while $V a l$ asserts that $\alpha$ is true in all structures[.] (Kreisel [1967: 153])

From this quote it is clear that Kreisel identifies an intuitively valid formula with one that is true in all structures, where structures are not limited to being found in the cumulative hierarchy. Kreisel's squeezing argument consists of the three premises:

1. $\forall \alpha: V \alpha \rightarrow D \alpha$.

2. $\forall \alpha: D \alpha \rightarrow$ Val $\alpha$.

3. $\forall \alpha: V a l \alpha \rightarrow V \alpha$.

Together these three premises squeeze the involved concepts together, and we conclude that $\forall \alpha: V a l \alpha \leftrightarrow V \alpha \leftrightarrow D \alpha$. The first premises is just the completeness theorem, and so the result holds modulo the assumption of completeness. The second premise is argued to be true by induction on the length of a proof, or what is in effect the same thing: inspecting that all axioms have this property and that the rules of inference preserve them. The third premise is to a certain extent obvious: Val ranges over all the structures that $V$ ranges over, so if $\alpha$ is true in all of the former it is most certainly true in all of the latter.

As Smith [2011: 26ff] points out, it is important to note that Kreisel's argument does not show that our intuitive concept of a valid sentence coincides with the modeltheoretic one. Of course, the phrasing of the points by Kreisel does not help. But the 
point is just this: Kreisel has already identified the intuitive notion of being a valid sentence with being true in all structures. The intuitive notion, as Kreisel uses the term, is not Tarski's common concept of validity, a concept about which Tarski says that "[i]ts extension is not sharply bounded and its usage fluctuates" (Tarski [1956a: 409]). The concept involved in the squeeze is not an intuitive notion in this sense. It is an informal notion which is not given in fully rigorous terms, but it is nevertheless definite enough for us to prove a rigorous result about it.

A squeezing argument for our informal notion would proceed in the same way. In fact, we have already discussed the three premises in turn in the previous section. The first premise is again the completeness theorem, so we are restricted to languages and model theories for which such is available. The second premise, in our attempted squeezing argument, is what we referred to as the deductive system being nice. These two premises together entailed that our model-theoretic definition is sound with respect to the informal notion we were out to model. In addition to this, we looked at a third premise asserting the completeness of our model-theoretic definition with respect to the informal notion. For all $\Gamma$ and $\alpha$.

(COM) If $\Gamma \models \alpha$, then $\Gamma \vdash \alpha$.

(DSL) If $\Gamma \vdash \alpha$, then $R_{A}(\alpha)$ is an I-consequence of $R_{A}(\Gamma)$.

(MCL) If $R_{A}(\alpha)$ is an I-consequence of $R_{A}(\Gamma)$, then $\Gamma \models \alpha$.

If all three premises are accepted we have a squeezing argument showing that our model-theoretic definition manages to model a relation which does in fact coincide with the natural language counterpart of being true in all possibilities in virtue of the meaning of the logical terms. Of course, for Etchemendy, these logical terms are just all terms, and since the model theory abstracts away from the exact meaning of the 
formal counterpart of some terms, we should not expect (MCL) to hold. For languages that have a nice complete deductive system, that is, (COM) and (DSL) hold, we get (MSL).

(MSL) If $\Gamma \models \alpha$, then $R_{A}(\alpha)$ is an I-consequence of $R_{A}(\Gamma)$.

This tells us that we have managed to represent all possibilities relative to the semantics of the terms and that our model-theoretic definition consequently does not overgenerate. However, what this cannot assure us of is that we have managed to represent only genuine possibilities relative to the semantics of all terms. If we allow for some terms to be treated categorematically, then there may be some models that do not represent a genuine possibility for some terms treated categorematically, but only for some interpretation of the terms so treated. ${ }^{87}$

\subsubsection{Consequence in Natural Language}

There can be no doubt that for Etchemendy there is a relation of logical consequence in natural language. In fact, Etchemendy quite clearly states that he believes that any (well-defined) system of representations, whether it be in the form of a language or not, comes equipped with a logical consequence relation (Etchemendy [2008: 283]). It is this notion of logical consequence, as it is found in natural language, which we have been concerned with capturing using model theory. Natural language has a certain semantics and it is in virtue of the interaction of this semantics with the world that certain things are excluded, or in other words, that certain things are guaranteed to be the case once certain other things are the case. The formal model, that is, the model theory, does not contain, in itself, the logical consequence relation as it is found in natural language, but

\footnotetext{
${ }^{87}$ Consider for an example once again the propositional variable $p$ with the reading $R_{A}(p)=$ ' $\mathrm{a}=\mathrm{a}$ '. A model assigning this the value 0 does not represent a genuine possibility relative to the semantics of the involved terms in ' $\mathrm{a}=\mathrm{a}$ ', but does so only relative to a different interpretation of $p$.
} 
does so only derivatively in the capacity of being a model of, in this case, the natural language phenomenon. ${ }^{88}$ When we have the notion of logical consequence as it obtains in natural language in mind, there is consequently a sense in which it is wrong to claim that $q$ follows logically from $p \rightarrow q$ and $p . q, p \rightarrow q$, and $p$ are just symbols of the formal language and as such no relations of logical consequence, in this sense, hold between them, as they are not yet members of the proper system of representations, that is, natural language. What we really mean to be conferring by saying that this is valid, on the present account, is that any two natural language statements that are acceptable readings of $p \rightarrow q$ and $p$ will logically entail the corresponding acceptable reading of $q$. And so, since $\rightarrow$ will have the same natural language meaning relative to all acceptable readings, one can say that all natural language statements of the form $p \rightarrow q$ and $p$ will entail natural language statements of the form $q$. As long as we stay clear on the proper content of this latter claim, there is of course no harm done. ${ }^{89}$

Etchemendy, or at least on our current understanding and interpretation of him, is therefore in direct opposition to the view that there is no logical consequence in natural language. This latter view is often motivated by the observation that there seems to be nothing in natural language which answers to the role of logical constant, and other phenomenon, which we do find in our formal languages. ${ }^{90}$ I should like to stress that it is unclear that the debate here rests on a fundamental difference in opinion, rather, it seems to me mostly to be a semantic debate as to what to properly

\footnotetext{
${ }^{88}$ That is not to say that the model-theoretic account could not capture a different logical consequence relation resulting from a different system of representations than natural language. Hence, we stress that in this case we are interested in the model theory as capturing the notion of logical consequence in natural language. This is of course also the case we are discussing throughout this thesis.

${ }^{89}$ We are of course talking from the perspective of Etchemendy as we have been interpreting him in this thesis. It can of course be denied that logical validity and logical truth applies to statements of natural language. A prominent example is Quine [1970] who takes logical validity and logical truth to be properties of schemata.

${ }^{90}$ Motivated by such observations, I stress. This is of course not the be all and end all of arguments purported to show that there is no logical consequence relation in natural language. See Glanzberg [forthcoming], Harman [1984], Sagi [2013].
} 
call 'logical consequence'. If we take the purely technical definition of a model-theoretic consequence relation as the prime example of logical consequence, then it is unclear that we can find a suitable candidate for this relation in natural language, simply because the model-theoretically defined relation possesses a given set of properties which we cannot find in natural language: most prominently, perhaps, that of each and every sentence of the (formal) language having a determinate form given in part by the logical constants, something which does not seem, at least on the face of it, to be the case for natural language. ${ }^{91}$

But, on the other hand, if we take natural language to be the vehicle for an interesting notion of logical consequence and set out to model it, then no such problems arise. Of course, there will always be the question of whether the formal side provides an adequate model of the phenomenon in question, but the model theory remains a model, and the answer to what corresponds to what remains completely within our reach in our capacity of being the modeler.

I do not take these two views to be in conflict, since it seems to be a point about what we are willing to call 'logical consequence'. If we take the formal notion of logical consequence to be the prime example, then this use of the concept logical consequence will - modulo the success of the arguments to that effect, of course - not have a counterpart in natural language. If, on the other hand, we choose to use logical consequence to refer to the notion of natural language arguments being necessarily truth preserving in virtue of the semantic properties of the involved terms, then we should see our formal notion of consequence as a model of this phenomenon. I take it that it is undeniable that there is such a concept: that is, a concept of the truth of some natural language statements making it necessary that certain other statements describe the world truly.

\footnotetext{
${ }^{91}$ By 'purely technical definition of a model-theoretic consequence relation' we mean the modeltheoretic definition as it is in itself, with its syntactically well-formed formulae, definition of truth in a model, and various technical properties, but without any mentioning of what the different parts represent or are meant to represent.
} 
Etchemendy's main insight is just that such a relation is interesting, so there should be no reason not to study it. ${ }^{92}$ Incidentally, Etchemendy also seems to believe that this relation (or at least a close variant of it) is what has been referred to historically as logical consequence and that such studies have already been carried out in a variety of languages, whether or not the theorists have been aware.

Any language, regardless of its expressive devices, gives rise to a consequence relation, a relation that supports inferences from sentences in the language to other sentences in the language. The study of this relation is the study of the logic of that language. When Carnap, Kanger, and Kripke studied languages with modal operators, they were doing logic. When Hintikka applied similar techniques to epistemic notions, he was doing logic. When Kaplan investigated indexicals, he was doing logic. Second-order logic is logic (though the Continuum Hypothesis may not be). And these are logic not in a derivative or secondary or lesser sense: they are studying precisely the same thing we study in first-order logic, though in languages with additional expressive resources. Logic is not limited, de jure, by the expressive power of the devices in the language, as the reductive account unavoidably implies, though it may be limited, de facto, by the clarity of those devices and the availability of techniques to study them. (Etchemendy [2008: 282])

But, and that is the main point, we do not have to accept these claims in order to accept that there $i s$ an interesting notion in natural language - that of a set of sentences guaranteeing the truth of another sentence - and set out to model it.

\footnotetext{
${ }^{92}$ In fact, as already explained, Etchemendy believes that there are many such interesting notions, one for each system of representations. However, in this thesis we focus on this notion as it is found in a particular system of representations: natural language.
} 


\subsubsection{Completeness by Default}

Griffiths [2014] has launched a criticism of the project of establishing the extension of model-theoretic definition of consequence relative to informal notions by the use of squeezing arguments. Such attempts, he argues, "get Kreisel wrong, and try to achieve more than he ever intended. This suggests that the model-theoretic definition should be viewed quite differently as an explication of our intuitions about logical consequence" [2014: 9]. The present section will try to make clear, as well as assess, these claims. Griffith is arguing in particular against the attempts of Shapiro and Etchemendy to adapt squeezing arguments. Here we will focus on Griffiths criticism of Etchemendy's framework.

Griffiths claims that Etchemendy's attempt to propose model theory as modeling a phenomenon of logical consequence which we find in natural language faces a dilemma. Etchemendy claims that the standard first-order model-theoretic definition of logical consequence undergenerates, while it can be shown, through a nice complete deductive system, that it does not overgenerate. We have already looked at this above. Either, Griffith claims, the formalization/reading procedure determining the relationship between natural language and formal language is determined by logical consequence, or

it is not. In the first case any claim as to the adequacy of the model theory as a model becomes trivially true, including both the claim that we have no overgeneration as well as the claim that there is no undergeneration. In the second case, the notion of formalization and reading is given independently of logical consequence, and "[i]t is not at all obvious what such a notion of formalisation would be, and it is certainly not how the process is usually understood" [2014: 14]. Griffiths points out that on the first horn of the dilemma we might have an assurance that our model theory does manage to capture the right informal notion, but once we reflect on this assurance we see that 
it is useless. If the result holds in this trivial manner, its truth cannot be due to the particular character of the informal notion. Hence, we could substitute any informal notion whatsoever and prove that it is coextensive with our model-theoretic notion. This, however, is clearly unacceptable as an assurance that we have managed to capture this particular informal notion. While I agree with this assessment, I will suggest that we have no reason to think that, though Etchemendy's notion is dependent on logical consequence, it is thereby dependent to an extent where the above becomes trivially true.

I seems to me that Etchemendy should accept the first horn of the dilemma. On the second horn of the dilemma Griffiths proposes to follow Quine's idea of a plausible account of formalization which is independent of consequence (Quine [1960: 160]). The idea is to obtain sentences of semi-ordinary language which are synonymous with the formal renderings of these sentences, and at the same time we can see these sentences as belonging to a well-behaved fragment of natural language. But, because of the way in which these different sentences are mechanically linked to one another, it is unclear that we have any counterexamples to the thesis of undergeneration, as Etchemendy's view requires there to be. The problem, Griffiths states, is that these counterexamples are to be found within the well-behaved fragment of natural language. Since the sentences of the well-behaved fragment of natural language are synonymous with sentences of the formal language, it is hard to see what these counterexamples could be, and it seems that it is unclear that we can maintain Etchemendy's claim that there is undergeneration going on. Part of the reason, Griffiths states, is that Etchemendy does not provide any examples of what he takes to be logically valid arguments whose counterpart are not declared valid in a standard model theory for first-order languages. As suggested above, 'All bachelors are unmarried' seems a prime example of a statement which is true in virtue of meaning in the requisite sense, but the formal counterpart of 
which, $\forall x(B x \rightarrow N x)$, is not true in all models. As such, I take this point to be quite independent of whether the process of reading/formalization here is dependent on, or independent of, logical consequence. To have our counterexample to Griffiths's claim it suffices that $R_{A}$ such that $R_{A}(\forall x(B x \rightarrow N x))=$ 'All bachelors are unmarried' is an acceptable reading.

However, I want to claim that Etchemendy should not view the process of reading and formalization as completely independent of logical consequence, that is, he should accept the first horn of the dilemma.

The relevant theorems are the following, for acceptable readings, $R_{A}$ :

(MSL) If $\Gamma \models \alpha$, then $R_{A}(\alpha)$ is an I-consequence of $R_{A}(\Gamma)$.

(MCL) If $R_{A}(\alpha)$ is an I-consequence of $R_{A}(\Gamma)$, then $\Gamma \models \alpha$.

The soundness result, (MSL), can be established by completeness of the nice deductive system for first-order languages, and will not concern us here. ${ }^{93}$ The interesting claim is that of completeness of the intuitive notion in natural language relative to the rigorously defined model-theoretic notion. The denial of completeness is the claim that there are acceptable readings, $R_{A}$, relative to at least one pair, $\langle\Gamma, \alpha\rangle$, s.t. $R_{A}(\alpha)$ is an I-consequence of $R_{A}(\Gamma)$ but $\Gamma \not \models \alpha$.

Griffiths claims that if we define the acceptable readings (and so conversely the formalizations as well) relative to the relation of consequence, then we are excluding the possibility of such readings by default. Here, I take it, is the idea:

Suppose the acceptable readings of $\Gamma$ are decided by looking at the properties that $\Gamma$ has relative to $\models$ and securing that analogous properties are possessed by $R_{A}(\Gamma)$ relative to I-consequence. Then it follows that $R_{A}(\Gamma)$

\footnotetext{
${ }^{93}$ I take it that if we can establish that (MCL) is not true by default, neither will (MSL) be true by default.
} 
entails $R_{A}(\alpha)$, by I-consequence, only if $\Gamma \models \alpha$, since this is just the very condition which we are putting on being an acceptable reading. But, the latter claim just is the claim that if $R_{A}(\alpha)$ is an I-consequence of $R_{A}(\Gamma)$, then $\Gamma \models \alpha$.

Now, I do not want to take issues with this piece of reasoning, as I do think that it is sound. The problem, as I see it from the perspective of Etchemendy is that the assumption is not true. It is clearly too strong, as it entails that ' $a=a$ ' is an unacceptable reading of $p$ in a propositional language, since the former is true in virtue of the meaning of the involved terms, and hence, on Etchemendy's view, is a logical truth. Yet we do not have $\models p$, so the properties of $R_{A}(p)=$ 'a $=\mathrm{a}$ ', in relation to I-consequence, are not decided by the properties of $p$ in relation to $\models$. $\forall p$, yet $R_{A}(p)$ is a logical truth. But it seems to me that the former is an acceptable reading of $p$; there just is no more truth functional structure to ' $\mathrm{a}=\mathrm{a}$ ' which can be given in terms of the logical constants for a propositional language. ${ }^{94}$ This, of course, is just a rehearsal of the upshot of treating some terms categorematically. We are not paying attention to their exact semantic behavior, and so since the properties of $R_{A}(\alpha)$, in relation to I-consequence, can depend on certain terms treated categorematically, we should naturally expect the former to come apart from the properties of $\alpha$ relative to $\models$.

Griffiths' claim relies on the fact that the kind of formalization and readings which are dependent on consequence should be given in a one-to-one manner, but this is exactly what we should not do if we want our model theory to illuminate the notion of consequence. Let us refer to the concepts of formalization and reading as the relation-

\footnotetext{
${ }^{94}$ At this point it is up to Griffiths to claim that his observations concern first-order languages only. I do not think the situation changes in any significant way, as 'All bachelors are unmarried' seems a perfectly acceptable reading of $\forall x(B x \rightarrow N x)$, but the former is true in virtue of meaning, whereas there are models making $\forall x(B x \rightarrow N x)$ false.
} 
ship between formal and natural language. The question then becomes whether, on Etchemendy's view, the relationship between formal and natural language is entirely dependent of the informal concept of logical consequence. Consider the formalization of 'If it is raining then the streets are wet' as $p \rightarrow q$. This clearly is not independent of logical consequence as it depends on a choice to see each model as giving the correct meaning of $\rightarrow$ as it mirrors the semantics of the natural language expression 'if... then...'. But accepting that this is the correct semantics for 'if... then...'- something which can be, and has been, questioned - is just to endorse all instances of modus ponens as valid. Giving the semantics of $\rightarrow$ clearly is not independent of logical consequence, but it does restrict the available readings. However, once the semantics of our constituent expressions are pinned down, and we have managed to meet the representational guidelines, we see that all acceptable readings of $\neg(p \wedge(\neg p \vee(q \wedge r))) \vee q$ will be true in virtue of meaning. This verdict, though certainly illuminating, is not meant to be independent of logical consequence or the relationship between formal and natural language. The former is meant to model (and illuminate) the latter.

I therefore find that Griffiths overstates the first horn of the dilemma. It is true that the relationship between formal and natural language is dependent on logical consequence: the semantic treatment of $\rightarrow$ and viewing it as the formal counterpart of 'if ... then ...' is, on Etchemendy's account, dependent on the fact that all modus ponens arguments in natural language are instances of logical consequence. In fact, this is the very fact which we are trying to model, so there should be no mystery here. However, it is untrue that the relationship between formal and natural language is, so to speak, completely dependent on logical consequence. The acceptable readings of a formula, $\alpha$, are not determined by securing that whatever properties $\alpha$ possesses relative to $\models$, the acceptable readings possess relative to the informal notion of consequence. There are acceptable readings of $p$, in a propositional language, such that $R_{A}(p)=$ ' $\mathrm{a}=\mathrm{a}$ '. And 
the decision to treat some terms categorematically then gives us a counterexample to (MCL): some models make $p$ false, since there are interpretations under which it is false. Hence, $\not \neq p$, but 'a $=\mathrm{a}$ ' is a logical truth in Etchemendy's informal sense. 


\section{PART III}

This chapter will concern itself with classifications of the different ways to view a model theory when used to model the notion of logical consequence. As we saw in the previous chapter, the theories which we looked at there allow for the models to both represent and interpret. In this sense the underlying model theory is both interpretational and representational. ${ }^{95}$

The standard way of seeing a model as providing an interpretation is given in (INT). If $I_{M}(\eta)=o$, for a model $M$ and the non-logical term $\eta$ of our language $L$, then $M$ simply interprets $\eta$ to refer to $o$. For example, if a model interprets the individual constant $a$ s.t. $I(a)$ is Obama, then that model interprets $a$ to mean what 'Obama' means, in the sense that $a$ is taken as a name for Obama, that is, as referring to

Obama. This way we are able to explain, by pointing only to certain features of the model itself - D, I and the relationship between the two-how certain interpretation of our language $L$ come about.

It is also reasonably straightforward to develop a theory of how a model represents, at least relative to a fully interpreted language, $L$. Consider $F a$ of $L$ as representing 'Obama is president of the USA'. Now, a model in which it is true represents a possibility in which Obama is the president of the USA, and a model where it is false represents

\footnotetext{
95 Though our use of these terms differ from the use of Etchemendy [1990, 2008]. But this is only to say that, as we commented in the previous chapters, Etchemendy uses the term interpretational semantics to refer to a model theory which endorses (INT) and (MRA). We have been referring to such a model theory as purely interpretational, since each model represents the actual world and the only differences between models are therefore interpretational. Here, we will use interpretational simply to mean that the models interpret - in an as of yet unspecified way - the non-logical terms.
} 
a possibility where he is not. Hence, it is through the truth values of the sentences of the language $L$ that the various models come to represent various possibilities. But the truth of $F a$ in a model is equivalent to the claim that $I_{M}(a) \in I_{M}(F)$. We are once again able to explain, by pointing only to features of $D, I$, and their relationship to one another, how it is that these models represent certain possibilities for the language $L$.

As we have argued in the previous chapter, the notion which we should see our model theory as capturing, in accordance with Etchemendy, is that of a valid argument being truth preserving in all possibilities under all interpretations of the non-logical terms. We would therefore like to make sense of our underlying models as both representing non-actual circumstances as well as providing the non-logical constants with interpretations. As we shall point out, in this case it is unclear that we can explain how a model manages to do so by pointing only to features of $D$ and $I$, and their relation to the language under investigation. This is not to say that we cannot see our models as both representing and interpretating, and we will present a way in which we can see our model theory as such. In this case, though, the fact that makes a model represent a certain possibility and interpretation is, so to speak, a modal fact about the model: a fact about how the model would have been related to reality had reality been different.

This shows that we can coherently see our model theory as capturing a phenomenon of being truth preserving in all possibilities under all interpretations of the non-logical terms. ${ }^{96}$ It also shows that if we want our model-theoretic definition of consequence to be both interpretational and representational, then there is a side of the story which

\footnotetext{
${ }^{96}$ I should like to stress that a negative verdict on this issue would show only that we cannot see the model theory as capturing the notion of being truth preserving in all possibilities under all interpretations of the non-logical terms. This would not show, in any way, that there is something incoherent about this latter notion, only that the techniques currently employed in standard model theory cannot capture this notion. This is important to keep in mind, as there is a crucial distinction between the coherency of the notion which the model-theoretic account of consequence is meant to capture and whether it does so adequately. A negative verdict on the latter question does not in itself have any bearing on the first.
} 
is not given by merely presenting the standard view of a model theory as a collection of objects each consisting of a domain and an interpretation function.

\subsection{A Categorization}

In the first part of the thesis we have seen examples of how we can view the underlying model theory when setting up a model-theoretic definition of logical consequence. As we saw, there are two essential parameters that can affect the truth of a statement: the meanings of the words, i.e., language, and the state of the world ${ }^{97}$ Differences between models can therefore be seen as differences in either parameter, none, or both. As we saw in the previous chapter, the standard understanding of models seems to allow for variation in both these parameters between models. Since we can have variation or no variation in two different parameters, we consequently get four $\left(=2^{2}\right)$ different ways of viewing the models of the underlying model theory.

\section{(A) Actual World \& Meaning Variance:}

On this view each model represents the actual world with an interpretation of the non-logical vocabulary. This is, of course, the kind of model theory that Tarski's original definition gives rise to according to Etchemendy, a view which sees the

\footnotetext{
${ }^{97}$ As work by Kaplan and others have shown, sometimes the meaning of a term and the state of the world is not enough to determine a truth value. The general idea is straightforward. Consider the statement 'I am hungry'. It is not enough to know the state of the world and the meaning of the statement in order to determine its truth, we must also know who said it. That is, a context is needed to determine the truth value. This, of course, relies on the fact that the meaning of the term ' $\mathrm{I}$ ' is separate from its reference, and that its reference is somehow determined by its meaning and a context (Kaplan [1977, 1989]). Kaplan distinguishes between character and content. Character, or linguistic meaning, determines the content of a statement in a given context. For example, if both I and Barack Obama utter 'I am hungry' then we have uttered a statement with the same character qua meaning. However, since the context makes my token of 'I' refer to me, and Obama's to Obama, the utterances have different content.

We will not be concerned with these complicating matters here. That is not to deny their importance. However, once we have a basic categorization we can come back and look at the more fine-grained details.
} 
models in accordance with (INT) and (MRA). In the first chapter of the present thesis we looked at Etchemendy's criticism of such an account, and we concluded, with Etchemendy, that the notion of logical consequence which it gives rise to just is not the standard understanding of logical consequence.

(B) Actual World \& No Meaning Variance:

On this view each model represents the actual world and no model reinterprets any of the non-logical terminology. Consequently there is no variation in any of the two parameters, and the model theory will contain only models that are elementarily equivalent. ${ }^{98}$ I take it that no one will want to see the underlying model theory falling into this category. We may note, incidentally, that the notion of consequence that results is just that of material consequence: having a false premise or a true conclusion. This squares well with Tarski's comments that when each term is treated as a logical term, his notion of consequence reduces to material consequence.

(C) Possible Worlds \& No Meaning Variance:

On this view we see each model as representing a way the world could have been and ask whether a given argument, given the actual semantic properties of the terms it involves, preserves truth on all such conceptions. There is therefore no reinterpretation of the non-logical terminology and we therefore require an interpreted formal language, $L$. A sentence $\alpha$ of $L$ is true in a model $M$ just when the model $M$ represents the world to be such that $\alpha$ is the case.

\footnotetext{
${ }^{98}$ Since all models represent the actual world relative to the intended interpretation of our language $L$, it follows that two models cannot disagree on the truth value of a sentence, since relative to this intended interpretation the sentence is either true or false, in accordance with our assumption that the language behaves classically. And whichever it is, all models must respect this truth value, and all models must consequently make the exact same sentences true.
} 
(D) Possible Worlds \& Meaning Variance:

On this view we see each extensional model as representing a way the world could logically have been as well as providing an interpretation of the non-logical terms. We therefore have two levels of variation: each model is meant to represent a possible circumstance as well as provide an interpretation for the non-logical terminology. In this chapter we ask, among other thing, how a model can meaningfully be seen to both represent and interpret in the requisite manner.

There are a few points worth making about this classification. First of all, the classification of model theories into the four different categories concerns what the models represent, not what the models themselves are made from. The classification does not concern how the models come to represent what they do, and so the four classifications are mutually exclusive. For example, if the models represent both possible circumstances and interpretations of the non-logical terms, then it is not the case that the models represent a possible circumstance as well as respects the meaning of the non-logical terms of our interpreted language $L .{ }^{99}$ Of course, when it comes to the question of how the models come to represent such, it may be relevant what the models themselves consist in, but from the perspective of what the models represent, this is a completely different question. It is perfectly compatible with category (A) that the models that capture this phenomenon consist only of merely possible objects, as well as it being perfectly compatible with (C) and (D) that all models are made up

\footnotetext{
99 Though there will naturally be models that are acceptable from more than one perspective. For the two mentioned categories, $(\mathrm{C})$ and $(\mathrm{D})$, in fact, every model that is acceptable from the perspective of (C) will also be acceptable from the perspective of (D), since the interpretation which $L$ actually enjoys is an interpretation of $L$. However, the converse is not the case. There may be models that are acceptable according to (D), but not according to (C). In general, though, the point is this: it may be that the very same model theory (consisting of exactly the same models) can be put to use in capturing all four of these categories in turn. But in accordance with what the models of the model theory are to represent, only one of (A), (B), (C), and (D) can be true of the model theory.
} 
from actual entities. ${ }^{100}$

Secondly, in chapter 2 we presented reasons for viewing Etchemendy's own theory of model-theoretic consequence as belonging to category (D). As we explained, the interesting concept for Etchemendy is still that of truth preservation in virtue of the semantics of all terms, but through focusing only on the semantics of certain terms we end up modeling a phenomenon belonging to category $(\mathrm{D})$, rather than $(\mathrm{C})$. I do think that this is the correct understanding of Etchemendy, but there is a related way of understanding Etchemendy, which Etchemendy himself at points seems to be endorsing. In order that someone will not object to my reading of Etchemendy by pointing out that Etchemendy had this alternative in mind, I find it necessary to make some brief comments about the issue.

The question is whether there is a way of understanding Etchemendy as endorsing a view of the model theory where models never interpret. We can find some evidence for this claim in some of Etchemendy's formulations in Etchemendy [2008]. According to this understanding, Etchemendy's modeling perspective is not that a valid argument is one such that it preserves truth in all possibilities under all interpretations of the non-logical terms. Rather, a valid argument is one such that it preserves truth in all possibilities in virtue of the semantics of all terms, but some models, as it just so happens, do not represent genuine possibilities relative to the semantics of these terms.

[T]he categorematic treatment of certain expressions ignores any specific meanings these expressions may have, if in fact they were drawn from an antecedently interpreted language. For example, if we start with the language of elementary arithmetic or a first-order language containing the

\footnotetext{
100 Of course, one might think that it would be very strange to hold that category (A) should be captured using models consisting of merely possible objects. One reason one might think this is that there is a straightforward way to capture category (A) that does make use only of actually existing objects. See next section.
} 
predicates $\cong$ and Triangle from our earlier example, there will be models that do not represent genuine possibilities - that is, possibilities consistent with antecedent meanings of these expressions. (Etchemendy [2008: 289])

The key in order to expouse the alternative understanding of Etchemendy's modeling perspective is the claim that "there will be models that do not represent genuine possibilities" [2008: 289]. The idea is that if $\cong$ is to mean that two objects have the same shape and Triangle that the objects it applies to are triangles, a model where $a \cong b$ and Triangle $(a)$ are both true while Triangle $(b)$ is false does not represent a genuine possibility. The point here is that we do not mention another interpretation enjoyed by these terms, and so the question about how the model provides such an interpretation does not arise. Instead, we think of the meanings of all terms, including the categorematic ones, as remaining fixed throughout the models, but since some models assign some categorematic expressions values that they could not have (for example, ' $\mathrm{a}=\mathrm{a}$ ' assigned the value 0 ), some models consequently do not represent genuine possibilities. Consider the model, $M$, such that $a \cong b$ and Triangle( $a$ ) are both true while Triangle(b) is false. We thus have the two different views of what this model represents:

- $M$ represents a genuinely possible circumstance in which Triangle and $\cong$ are given interpretations different from their intended interpretations.

- $M$ represents a circumstance in which $a$ has the same shape as $b, a$ is a triangle, but $b$ is not a triangle. Consequently, the represented circumstance is not a possible circumstance.

I think it is clear that Etchemendy wants to understand this particular model in accordance with the first option. As for the above quote, where Etchemendy claims that some models do not represent genuine possibilities, the key to understanding it 
is the continuation "that is, possibilities consistent with antecedent meanings of these expressions". Of course, if we are to respect the meanings of $\cong$ and Triangle, $M$ does not represent a genuine possibility. But Etchemendy never endorses that these models should respect the antecedent meanings of the terms treated categorematically.

[I]n a propositional language, we assign the value false to the atomic sentence $a=a$. This truth value assignment represents a genuine possibility for some atomic sentences, and since we are treating such sentences categorematically, the specific meaning of $a=a$ is irrelevant to the semantics. [2008: 289]

Relevant to our purposes is the fact that a model (what Etchemendy calls a truth value assignment) in which ' $\mathrm{a}=\mathrm{a}$ ' is false does represent a genuine possibility for at least some atomic sentences. But if the model represents a genuinely possible circumstance, then it cannot represent a circumstance in which ' $\mathrm{a}=\mathrm{a}$ ' is false, and we need another way of making sense of this. A true statement owes its truth to two parameters: the language and the world. To explain that ' $\mathrm{a}=\mathrm{a}$ ' is false, we cannot point to variation in the world as this would take us outside the realm of the possible, and the model would consequently not represent a genuine possibility. Therefore, we will have to point to differences in meaning to explain that ' $a=a$ ' is false. If, as Etchemendy states, the model represents a genuine possibility, then it follows that ' $\mathrm{a}=\mathrm{a}$ ' has a different interpretation than the one it actually enjoys. Etchemendy is clear on this as he explicitly states that it represents a genuine possibility for some atomic sentence.

Each model is meant to represent a semantically relevant circumstance for at least some expressions in those categories - or, if you will, for some interpretations of the expressions so treated. [2008: 289] 
The expressions so treated that Etchemendy is talking about here are the categorematic terms, and here we see Etchemendy clearly endorsing the view that the models represent possible circumstances for some interpretation of the categorematic terms. I therefore submit that Etchemendy does view the model theory as both interpretational and representational. The claims that could look as though they endorse that the model theory is only representational and that some models consequently represent impossible circumstances are all to be understood relative to holding the meaning of the categorematic terms fixed. But it is exactly such a fixation of the meaning of these terms that Etchemendy does not endorse. Models represent genuine possibilities, as Etchemendy himself puts it, "for some interpretations of the expressions so treated" [2008:289].

\subsubsection{On Interpretations}

The standard way, I submit, for a model to deliver an interpretation is via (INT).

(INT) A model, $M=\langle D, I\rangle$, interprets the non-logical term $\eta$ to have the extension $I(\eta)$

One thing we may note, is that in the formulation above, we speak only of the term $\eta$ having a certain extension, not of the meaning of $\eta$. The reason, of course, is that when we are concerned with extensional languages, we might claim there is nothing which we can equate with meaning proper. Meaning, it is claimed, is essentially an intensional notion, and often the meaning of a term is equated with what has now come to be known as an intension: a function from possible worlds to extensions.

As we have already seen, we are perfectly capable of making sense of category (A) above by endorsing (INT) and (MRA). As we also saw, the crucial flaw in this account is that it can only secure the actual truth preservation of a valid argument. Consider a 
fully interpreted formal language $L$, and let $R_{I}(\alpha)$ be the natural language counterpart of $\alpha$ according to this interpretation.

It is the presence of an intended model which secures that $R_{I}(\alpha)$ is true when $R_{I}(\Gamma)$ are all true, where by intended model we mean that the interpretations given to the non-logical terms in that model coincide with their interpretations under their intended reading, $R_{I}$. Consider the argument given as:

- Kasper is a unicorn and 2 is prime.

- Therefore, Kasper is a unicorn.

This is an acceptable reading of the following arguments, so consider the interpretation of what follows to be given by the above:

- $F a \wedge G b$.

- $F a$.

It is clear that the argument is valid. The intended model is such that $a$ is assigned to me, $b$ to the number $2, F$ to the set of unicorns and $G$ to the set of prime numbers. But assuming that are no unicorns in the actual world, $I(F)=\emptyset$, and the premise is false in the intended model. This, of course, is as it should be, since I am not a unicorn. But as we saw in a previous chapter, this is not enough to guarantee that if I had been a unicorn and 2 been prime, then I would have been a unicorn. The intended model secures only that the former is false, and all other models represent different interpretations of $F, G, a$, and $b$. Nothing here tells us anything about what would have happened had I been a unicorn.

We can now see how the models come to represent an interpretation of the nonlogical constants in the actual world. The objects of the domain of a model simply are 
the actually existing objects (or a proper subset of these) and the different interpretations are represented by the interpretation function $I$ assigning different extensions to the non-logical constants. All possible interpretations of the language are represented because for a given non-logical constant $\eta$, of the language, there is a model $M$ such that $I_{M}(\eta)=o$, for all objects $o$ of the appropriate kind. This is all we need in order to make sense of category $(\mathrm{A})$ above. ${ }^{101}$

\subsubsection{On Representations}

Suppose we want our models to capture the notion in category $(\mathrm{C})$. Here we are concerned with models representing other possible ways the world could have been, but relative to a language that does not vary in interpretation. Consider therefore an interpreted formal language $L$. We can now relatively easy represent possible ways the world could have been relative to this language. Consider the predicate Unicorn (with its obvious intended interpretation $R_{I}($ Unicorn $)=$ 'is a unicorn'). In order to represent a possible way the world could have been in which there are unicorns, we simply require that there is a model $M$ such that $I_{M}$ (unicorn $) \neq \emptyset$. In this way the sentence $\exists x \operatorname{Unicorn}(x)$ will be true in $M$, and so represent that unicorns do exist in the possibility which $M$ represents. ${ }^{102}$

On this way of viewing things, therefore, what does the representing is merely the

\footnotetext{
${ }^{101}$ We may note that this latter assumption - that for a given constant $\eta$, there will be a model where $I(\eta)=o$, for all objects $o$ of the appropriate kind - forces us to consider models beyond what we usually take into account. If two models are isomorphic, or at the very least elementarily equivalent, we normally consider ourselves justified in considering only one such. But from the present perspective this is unjustified, since all models must be present to survey all interpretations of the non-logical constants. This is not in itself a problem, since we rarely in the first place consider the models in our model theory one by one. We never consider this model, then that model, then that model, and so on, when deciding if something is a model-theoretic consequence of something else. The latter is usually shown from the meta-theory by considering only models where the premises are true, and proving that the conclusion must be too. Nevertheless, it is instructive to keep in mind that the underlying class of models, on this account, must have a staggering cardinality.

102 The natural language intended reading is just, in accordance with the fixed meaning of $\exists$, $R_{I}(\exists x \operatorname{Unicorn}(x)=$ 'There is at least one unicorn', or, in other words, 'Unicorns exist'.
} 
truth value of the sentences of the language. And since the truth values of these are given by the truth clauses, what makes this model represent that unicorns exist is simply that the interpretation of Unicorn is non-empty. It is therefore natural to see the set $I_{M}($ Unicorn $)$ to represent the set of unicorns in $M$. In fact, if we inspect the truth clauses for the non-logical terminology, we will see that if we let $I_{M}(\eta)$ represent, in $M$, the object that is in that possibility the intended interpretation of $\eta$, then these representational capacities will be reflected in the truth values assigned to the various sentences. For example, let $a$ be an individual constant of $L$ interpreted as a name for Barack Obama. Then, the model $M$ will represent Barack Obama as being a unicorn just when $I_{M}(a) \in I_{M}($ Unicorn $)$. The latter represents the set of unicorns in the possibility represented by $M$, and it is such that it includes $o$, which represents Obama. Consequently Unicorn $(a)$ will be true in $M$.

This picture relies on Unicorn having the interpreation of 'is a unicorn' attached to it, since it is relative to this interpretation that the members of $I_{M}($ Unicorn $)$ represent unicorns in a given model. We will thus need to have a fully interpreted language for this approach to work.

I submit that this is the standard way to see models as representing possible circumstances. Unfortunately, it is rarely explicitly discussed. We find a clear articulation of this view in Barwise \& Etchemendy [1989].

The function $f$ will assign various sorts of things to the basic expressions of Old Zealandish. For example, it will assign a subset of the domain $D$ to the unary predicate Planet. The set $f$ (Planet) will be thought of as representing the "extension" of the predicate in this model, that is, as representing the collection of planets (as opposed to moons, asteroids, and the like) in the solar system. Similarly the function $f$ will assign an element of $D$ to the name Earth, and this element will be taken as the representative 
of the Earth. [1989: 221]

' $f$ ' denotes, for Barwise \& Etchemendy, the interpretation function (which we call ' $I$ '). We see clearly in the formulation that the set $f$ (Planet) represents the set of planets. A model accordingly represents the Earth to be a planet if and only if $M \Vdash$ Planet(Earth), which in turn is the case if and only if $f($ Earth $) \in f($ Planet $)$. For models to represent in this way it is clear that we must work with a fully interpreted language. Models represent circumstances relative to the interpretation which the formal language enjoys. Consider for instance the formal sentence $F a$. A model $M$ s.t. $M \Vdash F a$ does not really represent anything. We might want to say that it represents a circumstance where $a$ is $F$. But there is a sense in which this really isn't representing anything at all. Given any possible circumstance, it would not be possible to say whether that circumstance is a circumstance in which $a$ is $F$, since $a$ and $F$ simply have no interpretation to respect by themselves. Only insofar as we have an interpretation of $\mathrm{Fa}$ in mind, say, as 'Barack Obama is Swiss', does the model $M$ represent a circumstance; in this case a circumstance where Barack Obama is Swiss.

For an interpreted language, $L$, we therefore have no trouble explaining how a model, viewed as a domain and an interpretation function, comes to represent a possible circumstance relative to this interpretation.

\subsubsection{On Representations and Interpretations}

As we have seen in the preceding two sections it is relatively straightforward how a model manages to provide interpretations of the non-logical terms as well as how a model manages to represent possible circumstances. For this reason we might expect it to be straightforward how a model manages to do both, but in fact it is not. The reason we cannot combine the two so easily is that the first delivers interpretations relative to a fixed representation of the actual world-but we would now like to represent other 
possible circumstances - whereas the latter represents possible circumstances relative to a fixed language - but we would now like to provide different interpretations for this language. ${ }^{103}$

Suppose we want an account of consequence that endorses models as both representing non-actual circumstances as well as providing interpretations for the non-logical constants. Suppose further that we see the models as interpreting in accordance with (INT). As we pointed out in the section on interpretations (and in chapter 2), we want a model for arguments of the following kind, where the premise is true because the model represents that unicorns exist.

- There are two unicorns (i.e., $\exists x \exists y(x \neq y) \wedge U n i \operatorname{corn}(x) \wedge U n i \operatorname{corn}(y))$.

- There is one unicorn (i.e., $\exists x U \operatorname{nicorn}(x))$.

As explained in chapter 2, a model where the premise is false is not enough to guarantee us that had there been two unicorns, there would also have been one. In particular, we want a model that represents that unicorns exist while delivering a certain interpretation of Unicorn: the intended interpretation. Suppose a model is to deliver this interpretation through (INT). $I_{M}($ Unicorn $)$ should then be a set of unicorns, since the way in which a model delivers an interpretation is by assigning it the appropriate extension, an extension which should, in this case, consist of unicorns. But it is not at clear that there are such objects available for us to build models out of. Under the assumption that there are no unicorns and that models, whatever else they may be, consist of actually existing objects, there are no unicorns available to be the extension of $I_{M}($ Unicorn $)$ in a model $M$. So this way of securing the interpretation of Unicorn

\footnotetext{
${ }^{103}$ All of this is relative to the assumption, which we are currently making, that there are no merely possible objects. If the latter exist, then, as we will explain below, we can see a model as prodiving an interpretation, via (INT), in another possible circumstance. Simply let the value of $I$ be the merely possible object from the possibility in question. However, we would like a theory of how models both represent and interpret under the assumption that models consist only of actually existing objects.
} 
in a model which represents unicorns as existing will ultimately have to rely on there being non-actual objects available - in this case unicorns - from which to construct the domains of the models.

I do not wish to claim that such a theory of how models both represent and interpret is unworkable. If one believes that there are such things as merely possible objects, then the straightforward way to capture a phenomenon of truth preservation in all possibilities under all interpretations of the non-logical terms, in terms of model theory, is to have the models contain these merely possible objects and assign interpretations to the non-logical constants in accordance with (INT). However, from the perspective of Etchemendy at least, who quite clearly maintains that models are built from objects that actually exist [2008: 286, n.17], we would prefer a different solution to the question of how models both represent and interpret. ${ }^{104}$

We want a theory of representations (for models) which allows us to represent circumstances involving unicorns and the like, without these models having to contain unicorns.

Kaplan [1978] suggests that it is quite possible for a model to represent a unicorn as existing without its domain containing a unicorn.

The elements of the universe of discourse of a model have an existence which is quite independent of whatever properties the model happens to tack onto

\footnotetext{
${ }^{104}$ In the footnote referenced above ([2008: 286, n.17]) Etchemendy does allow for models to be built from abstract, though still existing, objects. If we hold that unicorns, though not physically existing objects, nevertheless exist as abstract objects, we therefore seem to have a way of constructing domains that contain unicorns. However, it is unclear that this solution is satisfactory, since we presumably want interpretation for the language under which these abstract objects possess properties which they in fact do not possess. Consider the sentence 'There is a green unicorn' (i.e., $\exists x(\operatorname{Unicorn}(x) \wedge$ Green $(x)$ ). A model representing this to be true while delivering the intended interpretation for the language, in accordance with (INT), would have to contain an object that is both a unicorn and is green. But even if unicorns do exist as abstract entities, it is unclear that abstract entities are the kinds of things that have colors. However, a model $M$ should represent a possible way the world could have been where this is true under the intended interpretation by having $I_{M}($ Unicorn $) \cap I_{M}($ Green $) \neq \emptyset$.
} 
them. Suppose we want a model for the sentence of $L$ which asserts that there is exactly one thing and that it is a unicorn. A model for such a sentence must have a universe with only one element, and the extension assigned to the predicate "is a unicorn" must be the set consisting of that single element. And that is all that is required of the model. It is certainly not required that the single element of the universe of the model really be a unicorn. That would make the whole idea of the models unworkable (since there are no unicorns). The single element of the universe may be Jaakko Hintikka, or more likely, because logicians like their entities to exhibit a maximum degree of purity, it may be the null set, or singleton null. But, at any rate, it will be some definite entity which, in this model, is dressed as a unicorn. [1978: 97]

For this to be a viable account, we should have to seek an explanation of how the model comes to represent a given object, which per assumption is not a unicorn, as a unicorn. Kaplan continues:

Let me refer in a loose way to this kind of situation by saying that the entity in question is intrinsically Professor Hintikka or the null set or whatever, and extrinsically a unicorn. Most of the time logicians recognize a certain lack of significance in the intrinsic nature of the elements of the universe of a model (except, of course, with respect to identity and difference, i.e., how many of them there are) and focus their attention on isomorphism classes of models[.] [1978: 97]

Kaplan is here making a distinction between what we might want to call intrinsic and extrinsic properties (with respect to a model). Intrinsic properties are just the properties that the object actually has-being Jaakko Hintikka, for instance, in the 
case of Hintikka. The extrinsic properties are the properties that the model represents the object in question to have; for example, that of being a unicorn, or that of being identical to Jaakko Hintikka. Hintikka could of course be used to represent himself in a model, but it does not follow from this that the extrinsic and intrinsic properties of Hintikka (relative to that model) coincide. Perhaps the model is one that represents Hintikka as being Swedish, whereas, in actual fact, he is Finnish. So even if the objects are available for us to build models out of, the extrinsic properties are still added to these objects as an, so to speak, extra layer by the models.

For our present purposes we are therefore interested in finding a way to make models represent extrinsic properties that are different from the intrinsic properties of the objects of the domains. As we explained above, having the models deliver interpretations in accordance with (INT), the interpretations will depend solely on the intrinsic properties of the objects of the domains, since $M$ interprets $\eta$ as having the extension $I_{M}(\eta)$ in $M$, and the extension of $I_{M}(\eta)$ is an object having certain intrinsic properties, which are meant to represent the interpretation of $\eta$. For example, if $I_{M}(F)$ is the set of dogs in the domain, then $M$ gives the interpretation to $F$ which corresponds to 'is a dog' in natural language. We can therefore not, on this simple approach, represent interpretations where the extrinsic properties are different from the intrinsic properties of the given objects.

On the other hand, if we let our models represent different ways the world could have been through the relationship between an interpreted formal language and the properties of $D$ and $I$, then the intrinsic properties of the models will not matter. The extrinsic properties of the objects of the models are mapped onto them in a very straightforward sense: if $I_{M}($ Unicorn $)=\left\{o_{1}, o_{2}\right\}$, then $o_{1}$ and $o_{2}$ both have the extrinsic property, in $M$, of being a unicorn. But since these extrinsic properties are mapped onto the objects relative to a fixed interpretation of $L$, we cannot, in the same 
straightforward manner, explain how different extrinsic properties than those decided by the interpretation of $L$ are mapped onto those objects: that is, we cannot in the same straightforward manner explain how the models also give different interpretations to the language.

\subsubsection{A Possible Solution}

In this section we will present a solution to how we can see the models as both representing other possible ways the world could have been as well as providing interpretations for the non-logical constants. The solution is due to Menzel [1990]. In this paper Menzel is concerned with developing an actualist semantics for a modal language. The problem, which Menzel faces, is how to account for the truth of certain modal statements without making reference to the merely possible objects which these statements seem to be about. In order to do so, Menzel develops a theory of how the indices of a modal model can represent non-actual circumstances without the associated domain containing non-actual objects. ${ }^{105}$ The solution which Menzel proposes therefore carries over to our present concern, since we can view each index of a modal model as giving rise to an extensional model, in the sense of having a domain and an accompanying interpretation function.

As we explained in the previous section, there seems to be no fact of the matter about the language $L$ in relation only to a model, viewed as a domain and an interpretation function, which makes a certain model represent a certain possible way the world could have been along with an interpretation of the non-logical constants - at least for certain pairs of possibilities and interpretations. Menzel's solution does not go against this observation. What makes a model represent a certain possibility and

\footnotetext{
105 The concept of an index and a modal model will be explain in the next chapter. If the reader is unfamiliar with these concepts, I suggest reading chapter 4 before returning to read the remainder of this chapter.
} 
interpretation on Menzel's account is a modal fact: a fact about the relationship that a given model would have had to reality had reality been different in certain respects. We therefore have no reduction of the notion of a possibility to that of actual objects and their relations. We may note that this is perfectly acceptable from Etchemendy's modeling perspective, as the model-theoretic account of logical consequence is meant to rely on a notion of a possible way the world could have been relative to the semantics of the terms, not analyse this notion in terms of more basic notions.

Menzel [1990: 371] motivates his account by noting, quite correctly, that he could have had a third child, whereas in actual fact he only has two children. Consider the sentence 'Christopher Menzel has three children'. We may naturally formalize this sentence as $\exists x, y, z, u((x \neq y) \wedge(x \neq z) \wedge(x \neq u) \wedge(y \neq z) \wedge(y \neq u) \wedge(z \neq u) \wedge(x=$ Menzel $) \wedge \operatorname{IsAChildOf}(y, x) \wedge \operatorname{IsAChildOf}(z, x) \wedge \operatorname{IsAChildOf}(u, x))$ (henceforth $\varphi(x, y, z, u))$. Consider a model for this sentence, $M$, such that $D_{M}=\left\{o_{1}, o_{2}, o_{3}, o_{4}\right\}$, $I_{M}($ IsAChildOf $)=\left\{\left\langle o_{2}, o_{1}\right\rangle,\left\langle o_{3}, o_{1}\right\rangle,\left\langle o_{4}, o_{1}\right\rangle\right\}$, and $I_{M}($ Menzel $)=o_{1}$. The trouble with this model is that unless one of $\mathrm{O}_{2}, \mathrm{O}_{3}, \mathrm{O}_{4}$ is the third child of Menzel, whom he might have had, this model cannot be the intended model for the intended interpretation of the language. But the model does just as well as the intended model would have done had it existed, when the aim is solely to make the target sentence $\varphi(x, y, z, u)$ true. The reason is that there is a certain relationship between the model, $M$, and reality as it would have been had Christopher Menzel had a third child. This relationship can be specified through an embedding function $\mu$. Suppose Menzel had a third child. In that possible way the world could have been there would be an embedding from the objects of the model $M$ to Christopher Menzel's three children, call them $c_{1}, c_{2}$, and $c_{3}$, respectively, such that $\mu\left(o_{1}\right)=$ Christopher Menzel, $\mu\left(o_{2}\right)=c_{1}$, $\mu\left(o_{3}\right)=c_{2}$, and $\mu\left(o_{4}\right)=c_{3} \cdot{ }^{106}$ Furthermore, this embedding respects the intended

\footnotetext{
${ }^{106}$ In order to secure that this embedding would have existed in the possibility in question, we further
} 
meaning of IsAChildOf in the sense that in that possible way the world could have been $\left\langle O_{1}, O_{2}\right\rangle \in I_{M}(I s A C h i l d O f)$ iff $\mu\left(O_{1}\right)$ is a child of $\mu\left(O_{2}\right)$. We can therefore see the model $M$ as representing a possible way the world could have been where Christopher Menzel has three children, and where the interpretation of the non-logical term IsAChildOf is the intended interpretation. What makes the model represent this possibility and interpretation is a modal fact: a fact about a certain embedding, $\mu$, which, though it does not necessarily exist, would have existed had the possibility in question been actual. ${ }^{107}$ Note, again, that this is perfectly acceptable from our present modeling perspective, as we are not aiming to analyse the notion of a possible way the world could have been, in terms of more basic notions, but are merely presupposing them.

We may also note that the same model, $M$, does not have to respect the intended interpretation of the non-logical term IsAChildOf, but can give it another interpretation. Consider a possible way the world could have been where John Locke, rather than Immanuel Kant, wrote the three critiques: Critique of Pure Reason, Critique of Judgment, and Critique of Practical Reason. In this possible way the world could have been there would have existed another embedding function $\mu^{\prime}$ such that $\mu^{\prime}\left(o_{1}\right)=$ John Locke, $\mu^{\prime}\left(o_{2}\right)=$ Critique of Pure Reason, $\mu^{\prime}\left(o_{3}\right)=$ Critique of Judgment, and $\mu^{\prime}\left(o_{4}\right)=$ Critique of Practical Reason. The model $M$ can therefore also be seen as representing a possible way the world could have been where John Locke wrote the three critiques as well as providing an interpretation to the non-logical term IsAChildOf which is given in English as 'is authored by'. This latter interpretation comes about through want to claim that our models consist of necessary objects, such that the function $\mu$ is well-defined. Menzel [1990: 372] suggests viewing the models as built up from pure sets.

${ }^{107}$ For some models representing the actual way the world could have been, or other possible ways the world could have been involving only actual individuals, the embedding functions in question do actually exist. However, in general we can have no guarantee that a given embedding does actually exist. The embedding, $\mu$, above is a prime example. It does not actually exist, since there is no object to be the image of $o_{4}$ under $\mu$, but it would have existed in the possibility where all the images of the function do exist. 
the fact that in the possibility in question: $\left\langle O_{1}, O_{2}\right\rangle \in I_{M}(I s A C h i l d O f)$ iff $\mu^{\prime}\left(O_{1}\right)$ is authored by $\mu^{\prime}\left(\mathrm{O}_{2}\right)$. Consequently, since $I_{M}(\mathrm{Menzel})=o_{1}$, Menzel is interpreted as a name for John Locke, and the target sentence $\varphi(x, y, z, u)$ expresses that John Locke is the author of three works, something which is represented as being true.

In general, then, the same model can represent many different possible ways the world could have been as well as provide many different interpretations to the nonlogical terms, in accordance with the following:

Let $\mu$ be an (injective) embedding that exists in a possible way the world could have been mapping members of $D$ into objects of this possible way the world could have been. Then:

i. For each individual constant, $a$ of $L, M$ interprets $a$ as $\mu\left(I_{M}(a)\right)$, whatever object that may be;

ii. For each n-ary predicate, $P$ of $L, M$ interprets $P$ as $\left\{\left\langle\mu\left(o_{1}\right), \ldots, \mu\left(o_{n}\right)\right\rangle \mid\left\langle o_{1}, \ldots, o_{n}\right\rangle \in\right.$ $\left.I_{M}(P)\right\}$, whatever property or relation has that extension; and

iii. $M$ represents the possible way the world could have been where the members of $\left.\mu\right|_{D}$ are so propertied and so structured. ${ }^{108}$

As we have already noted this means that the same model can represent many different possible ways the world could have been as well as many different interpretations of the non-logical constants. This is clear from the fact that for any given model there will be many different possible ways the world could have been for which there would exist the requisite embedding. And in each such possible way the world could have been, there will also be many different ways of defining this embedding, thus giving rise to many different interpretations of the non-logical constants.

\footnotetext{
${ }^{108}$ I owe large parts of this formulation to Christopher Menzel in written communication.
} 
We can now see how a model can come to interpret a given predicate $F$ as the formal counterpart of 'is a unicorn' as well as represent the extension of this as being non-empty. Let $M$ be a model. We then require that there is a possible way the world could have been where at least one unicorn exists as well as an embedding $\mu$ such that for $o \in D: \mu(o)$ is a unicorn iff $o \in I_{M}(F)$. By the clauses above, $M$ interprets $F$ as having the extension $\left\{\mu(o) \mid o \in I_{M}(F)\right\}$, which is a set of unicorns. ${ }^{109}$

We thus have a working theory of how a model can be seen as both representing a non-actual circumstance as well as provide an interpretation for the non-logical constants. In developing this theory we relied on certain modal facts about the models. Facts about how the model would relate to reality had reality been different. We can therefore see how our model theory is able to capture the blended notion of consequence, as envisioned by Shapiro, and Etchemendy's idea of allowing for some terms to be treated categorematically, thus capturing a notion of truth preservation in all possibilities under all interpretations of the terms.

\footnotetext{
${ }^{109}$ Note that we use the formulation that $M$ interprets $F$ as having the extension of a set of unicorns. This makes the natural language counterpart of $F$, in this model, that of 'is a unicorn', in the sense that it will truly apply to the members of $\left\{\mu(o) \mid o \in I_{M}(F)\right\}$. This was the interpretation which we wanted to secure. If we further wish to claim that $F$ is interpreted as the property of being a unicorn, we would have to further require that all and only unicorns that exist in this possibility are the image of some $o$ under $\mu$ for which $o \in I_{M}(F)$. Otherwise the set $\left\{\mu(o) \mid o \in I_{M}(F)\right\}$ may be, in this possibility, a proper subset of the set of unicorns that exist, and so we cannot equate this with the property of being a unicorn. The problem, of course, is that it is unclear that we even can individuate properties in a purely extensional setting. The notion of a property is often inviduated in an intensional context: as the class of things that would fall into the extension, not merely the things that happen to do so. In general, then, it seems that for a given interpretation of an n-ary predicate there will be more than one property compatible with the extension which the predicate receives. We may also note that many interpretations may be unnatural from the perspective of natural language. There may be an interpretation which assigns to the predicate $G$ The Eiffel Tower, my glasses, and the copy of Lewis' On The Plurality of Worlds currently sitting on my shelf. This may be considered unnatural in the sense that we have no predicate in English which applies truly to all and only these things, but also in the sense that it is hard to pinpoint which property this set is to be equated with. None of these, however, are problems that should bother us. We seeked a way to make sense of models interpreting the non-logical constants in terms of assigning them extensions, quite independently of whether these extensions can be expressed with a natural language predicate or whether we can equate them with any properties. The above approach works perfectly fine for that purpose.
} 


\section{PART IV}

In this chapter we will look at model theories for modal languages. We will argue that our findings from the previous chapters - that the model theory should be (or at least can be) seen as capturing a notion of being truth preserving in all possibilities under all interpretations - still apply in the modal case, and we will use this to explain how Kripke Semantics can be seen as capturing this notion of a valid argument even in a language augmented with a model operator for logical necessity.

There is a criticism of Kripke Semantics in the literature which claims that Kripke Semantics is not the correct semantics for a certain reading of $\square$. This criticism of Kripke Semantics has it that it is unable to deliver the correct truth conditions for when this is understood as logical necessity. The problem according to the critics- most prominently Cocchiarella [1975, 2002] and Hintikka [1980, 1982] - is that any set whatsoever is allowed as the index set, $W$, in a Kripke Model, and hence it is not required that for $\square \alpha$ to be true, $\alpha$ must be true in all logically possible worlds, since not all possible worlds, or accurate representations of them, need be in $W$ (Hintikka [1982: 90ff]). Instead, Cocchiarella and Hintikka propose that the quantifier in the truth clause for $\square$ should range over all models for a first-order language, or all valuations for a propositional language. ${ }^{110}$

In this chapter I will argue that there is an interesting notion of a valid argument-

${ }^{110}$ Cocchiarella [1975] and Hintikka [1980, 1982] are concerned with quantified languages. Cocchiarella [2002] explicitly endorses an approach to propositional languages where the truth clause for $\square \alpha$ ranges over all possible valuations of the atomic sentences. 
that of being truth preserving in all possibilities under all interpretations of the nonlogical constants - which is captured by Kripke Semantics for a language containing an operator for logical necessity. There are a few things to note about this claim. Firstly, I do not necessarily see this claim as being counter to the criticism launched by Hintikka [1980, 1982] and Cocchiarella [1975]. The reason is of historical importance: both authors are discussing a particular notion of logical necessity. Cocchiarella [1975: 13] clearly states that the criticism of Kripke Semantics is modulo the claim that we want a notion of logical necessity which is acceptable from the perspective of a conceptual framework typified by logical atomism. I do not wish to claim that this is mistaken. In fact, as we shall see, if we attempt to unify Cocchiarella's alternative semantics with the idea that we are out to capture a notion of being truth preserving in all possibilities under all interpretations, it becomes quite clear how the resulting semantics relies on a notion of absolute logical form, which is also the backbone of a theory of logical atomism. When Hintikka [1980: 90-91] criticizes Kripke Semantics for not being able to capture the correct notion of logical necessity in the object language, it also seems Hintikka has a particular concept of logical necessity in mind, a concept which can be traced back to the work of C.I. Lewis, whom Hintikka especially mentions, and has been studied extensively in the work of Carnap and others. ${ }^{111}$ I do not wish to claim that all of these authors have the same concept of logical necessity in mind, only that the context relative to which Hintikka makes his claim may be that the correct notion of logical necessity, which Kripke Semantics does not capture, is a notion which is highly influenced by these writers and so not necessarily the concept of being true in all possibilities in virtue of the semantics of the terms.

My claim is that there is an interesting notion of logical necessity - the necessity with which a valid argument preserves truth - given as being truth preserving in all

\footnotetext{
${ }^{111}$ See, for example, Barcan [1946], Carnap [1946, 1947], Hintikka [1961], Kanger [1957], Lewis [1918], Lewis \& Langford [1932], Prior [1957], Quine [1947], Segerberg [1971].
} 
possibilities under all interpretations of the non-logical terms, which Kripke Semantics does capture. In arguing this I find it instructive to compare Kripke Semantics to the alternative semantics developed by Hintikka [1980, 1982] and Cocchiarella [1975], as well as point out why the criticism is mistaken from a standpoint where we want the model theory to capture this notion. But the reader should keep in mind that we are not necessarily objecting to the claims made by Hintikka and Cocchiarella, rather, we are arguing for a conditional claim: if we want our model-theoretic definition of consequence to capture, in a language augmented with an operator for logical necessity, the notion of being truth preserving in all possibilities under all interpretations of the non-logical terminology, then it is the approach taken in a standard Kripke Semantics, and not the alternative semantics developed by Hintikka and Cocchiarella, which is the correct one.

This chapter aims to show that, from the point of view of modeling a notion of truth preservation in all possibilities under all interpretations, the criticism of Kripke Semantics is mistaken in that it neglects to pay attention to the interpretational character of the informal notion which we are out to model. This is not to say that there is no use at all for the alternative semantics and its accompanying notion of a valid argument. Such a notion can be motivated from a logical atomist perspective, something which is explicitly done by Cocchiarella [1975]. But from our present perspective, this suggests that it is not Kripke Semantics that incorporates material content into the semantics of $\square$, as Cocchiarella explicitly claims ([1975: 13]), but rather, it is the envisioned alternative which incorporates metaphysical content into the semantics of $\square$. And, as we shall look at, this severely limits the explanatory power of our theory of consequence, in a way which we do not, presumably, want it to be limited.

In the previous chapter we looked at how a model can be seen to interpret, or assign 
a meaning to, the non-logical terminology. But there is something odd about viewing an extensional model as assigning a meaning to the non-logical terms. Meaning, as it is normally understood, is something different from a referent - whether that referent be a truth value, a set, an object, or what have you. Consider for example a propositional language. It is hard to reconcile the assignment of a truth value to an atomic sentence with the assignment of a meaning. Rather, the meaning of a declarative sentence is often identified with a proposition, and a proposition is in turn identified with a set of worlds. For this reason, we see Cresswell [1972] suggest that the propositional calculus has a misleading name.

Indeed one might say that the name 'propositional calculus' is misleading since the values of the sentences are not propositions but simply either 'The True' or 'The False'. Or one might say that as far as the propositional calculus is concerned propositions (i.e., values of sentences) just are truth values and that there are therefore only two propositions. [1972: 4]

The point here is well taken. There is nothing in a truth value which determines what proposition is expressed - unless, of course, as Cresswell notes, we admit that there are only two propositions - and hence, since extensional models assign only truth values it is unclear that an extensional model theory for a propositional language involves propositions, at least as we normally think of them: as the meanings expressed by certain natural language statements.

Of course, Cresswell's reason for bringing this up is to suggest a picture on which we can identify the proposition expressed by a certain sentence with its truth conditions, in the following way:

What is perhaps more plausible is to say that two sentences have the same meaning if they have the same truth conditions. I.e., if every state of affairs 
which would make the first true would also make the second true and vice versa. The meaning of a sentence can then be thought of as the set of states of affairs or possible worlds in which it would be true. In this way, provided we have the notion of a possible world, we can define a proposition as a set of possible worlds and say that each sentence of our language is to be assigned a proposition, i.e., a set of possible worlds. The sentence is to be regarded as true in a possible world if that possible world is in the set assigned to the sentence and false if it is not. [1972: 4]

Two sentences mean the same if they express the same proposition, that is, if they are assigned the same set of worlds. There therefore is a sense of definite meaning which can be defined if we have available the notion of a possible world and the set of all such worlds, simply identify the meaning of a sentence with the proposition it expresses which, in turn, is identified with the set of worlds at which it is true. What this shows, therefore, is that a model theory for a modal language seems to have, at least on the face of it, the resources needed to see a full model as assigning a meaning proper to the non-logical terms, since each model is standardly seen as containing a set of possible worlds. Hanson \& Hawthorne [1985] have proposed a model theory for modal language which is based on this intuition. As they argue, if each model is based on the frame which contains all possible worlds, we can see each interpretation (that is, model built on this frame) as assigning a meaning proper to the non-logical terms. We will look at this proposal later, where we will point out that H\&H's treatment of logical necessity suffers from the same limitations as does Cocchiarella and Hintikka's proposals. 


\subsection{I-models \& E-models}

When we consider a model theory for a modal language we introduce even more entities that could be seen as representations of something or other, namely the indices of our models along with the valuations given there. To avoid confusion as to the kind of models we are talking about let me reserve the term 'E-model' for an extensional model in the sense in which we have been discussing these in the first three chapters. An Emodel, for a first-order language, consists of a domain and an interpretation function assigning appropriate denotations from that domain to the non-logical vocabulary of the language.

Now, when we move to consider modal languages E-models will no longer be able to do the job. Let us therefore introduce the notion of an I-model ("I" for intensional - "E" for extensional).

(I-model) An I-model for a first-order modal language is a tuple $\langle W$, @, $R, D, \psi, I\rangle$ s.t. $W$ is a set, @ $\in W, R$ is a relation on $W$; that is $R \subseteq W \times W, \psi$ is a function that returns, for a given $w \in W$, a set of objects $\psi(w) \subseteq D$, and $I$ is a function that assigns, for a given $w \in W$, a function that assigns appropriate denotations for the non-logical terminology of our language drawn from $D .^{112}$

As the reader will have noticed, our definition of an I-model is equivalent to that of a standard Kripke Model. According to the standard explanation we are to think of $W$ as a set of possible worlds; @ as the actual world; $R$ as a relation of relative possibility: if $\left\langle w, w^{\prime}\right\rangle \in R$ then $w^{\prime}$ is possible relative to $w$. For $w \in W, \psi(w)$ is to be thought of as the domain of $w,{ }^{113}$ and for each $w \in W, I(w)$ is an interpretation function assigning

\footnotetext{
112 The latter condition can be strengthened to demand that for each $w \in W, I$ assigns a function $I(w)$ which assigns appropriate denotations to the non-logical terminology drawn from only $\psi(w)$. One could have various motivations for such a restriction, but we will not discuss these issues.

${ }^{113}$ We will sometimes use the notation ' $D_{w}$ ' for the value of the function $\psi$ on $w . D_{w}$ is therefore a notational equivalent for the domain of $w, \psi(w)$.
} 
appropriate denotations to the non-logical terminology at $w$.

We will use the notion of a I-model to refer to any kind of model which is used in a model theory for a modal language. Common to all such conceptions of I-models is that a model consist of more than just one interpretation function for the language, where by an interpretation function we mean a function assigning appropriate denotations to the language. Whether these interpretation functions are to be seen as interpreting or representing will concern us shortly. We should note that the interpretation functions, as we are currently speaking of them, are associated with the indices of a full I-model. Whether or not they interpret or represent is therefore not Etchemendy's distinction between interpretational and representational semantics, as this distinction pertains to what a single model represents, though Etchemendy's distinction is of course related. A Kripke Model, given as $\langle W$, @, $R, D, \psi, I\rangle$, is the standard example of an I-model in the literature. To define the truth of a formula containing free variables we need to define an assignment function, as in the extensional case. A assignment function, $f$, assigns to each variable a member of $D$. Let $t$ be a term, that is, either an individual constant or a variable. We define the denotation of $t$ relative to $f, \operatorname{den}(t)_{f}$, as $\operatorname{den}(t)_{f}=I(w)(t)$, if $t$ is an individual constant; $\operatorname{den}(t)_{f}=f(t)$, if $t$ is a variable. The notion of truth in a model at an index $w$ is then defined relative to an assignment function, $f$, in the following way:

(at) $F t$ is true at $w$ (relative to $f$ ) iff $\operatorname{den}(t)_{f} \in I(w)(F)$.

$(=) t_{1}=t_{2}$ is true at $w$ (relative to $f$ ) iff $\operatorname{den}\left(t_{1}\right)_{f}=\operatorname{den}\left(t_{2}\right)_{f}$.

$(\neg) \neg \alpha$ is true at $w$ (relative to $f$ ) iff $\alpha$ is not true at $w$ (relative to $f$ ).

$(\wedge) \alpha \wedge \beta$ is true at $w$ (relative to $f$ ) iff $\alpha$ is true at $w$ (relative to $f$ ) and $\beta$ is true at $w$ (relative to $f$ ). 
$(\forall) \forall x \alpha$ is true at $w$ (relative to $f$ ) iff $\alpha$ is true at $w$ (relative to $f[x / a]$ ) for all objects $a \in \psi(w) .{ }^{114}$

Since these are just the standard truth clauses for our logical terminology, the truth or falsity of a non-modal formula at a certain member of $w$ is decided just like truth or falsity in an E-model. ${ }^{115}$

The truth clause for formulas containing modal operators is defined relative to all accessible indices in the following recursive way:

( $\square) \square \alpha$ is true at $w$ (relative to $f$ ) iff $\alpha$ is true at all $w^{\prime}$ (relative to $f$ ) s.t. $\left\langle w, w^{\prime}\right\rangle \in$ $R \cdot{ }^{116}$

Intuitively this is to reflect the fact that a formula is necessary at a world $w \in W$ if and only if it is true at all worlds $w^{\prime} \in W$ that are possible relative to $w$.

To have the notion of truth in a model we can simply pick out a special member of the set of indices. In a standard Kripke Model, for example, we say $\alpha$ is true in $\langle W, @, R, \psi, D, I\rangle$ iff $\alpha$ is true at @ ${ }^{117}$ As shall become clear later, we choose to have 114 The assignment function variant $f[x / a]$ is defines as the assignment function such that $f[x / a](y)=$ $f(y)$, for all $y \neq x$, and $f[x / a](x)=a$.

In the above presentation we have allowed for varying domains; that is, for $w, w^{\prime} \in W$, we may have $\psi(w) \neq \psi\left(w^{\prime}\right)$. As an upshot this means that the quantifiers as evaluated at $w$ range only over objects in $\psi(w)$ and that there may be some object in $D$ that is not in the range of the quantifier as evaluated at a given world. There are substantial metaphysical debates in the vicinity, since allowing for varying domains we can effectively invalidate the Barcan Formula and other formulae whose validity or invalidity is greatly involved, or depends on, the answer to substantial metaphysical questions. I do not wish to take sides in this debate. The definition given is but one of many. A single domain model theory in which the Barcan Formula is valid can be obtained from this definition by demanding that $\psi$ be a constant function such that for all $w, \psi(w)=D$.

115 Technically, of course, there are no E-models in an I-model, and for this reason there will be some variation in the content of the truth clauses for our logical terminology when turning from E-models to I-models, which is forced upon us by the technicalities. What matters for now, though, is not how this is done, but that it can be done.

116 Since the examples which we will use in this thesis do not contain free variables, we will in general omit to make reference to the assignment function, $f$, unless it is strictly needed.

${ }^{117}$ It should be noted, that for purposes of the output of the semantics, a designated index at which to decide truth in a model is actually superfluous given that the language does not contain any operators whose truth clause make reference to a specific member of $W$. The actuality operator is a standard example. $A \alpha$ (read: 'it is actually the case that $\alpha$ ') is true at $w \in W$ if and only if $\alpha$ is true at $@ \in W$. 
a designated index in each model in order to provide a perspective from which a possible way the world could have been is represented along with an interpretation of the non-logical terminology.

The question that is of immediate concern is how to philosophically understand a collection of I-models used to define the notion of logical consequence and validity for a given language in the standard manner. ${ }^{118}$ I will suggest that we are to understand differences in truth value of sentences between I-models as differences in the interpretation enjoyed by the non-logical terms from the perspective of a way the world could have been, whereas differences in truth value atthe indices are to be understood as differences in how the world could have been, that is, as representing that the facts could have been different relative to a given interpretation.

\subsubsection{Pure vs. Applied Semantics}

Plantinga [1974] draws a distinction between what he calls 'pure semantics' and 'applied semantics'. The idea is that a pure semantics is simply a collection of objects of some sort or other with a structure defined on them and a relation between these structures and the terms of our formal language. Though the distinction is not limited to semantics for modal languages, and applies equally well in the extensional setting, we will focus on the former. In this relation, we can see a pure semantics as simply a collection of I-models for a language. When we defined an I-model above we were told to intuitively think of $W$ as a set of worlds and the members of $\psi(w)$ as the objects existing in $w$, and so on. But really, the definition is just given in set-theoretical terms and for this purpose the members of $W$ and $\psi(w)$ for a given $w$ could be anything:

In the absence of such operators a sentence will be true in all models at @ if and only if it is true at all indices $w$ in all models.

118 By 'standard manner' I mean defining logical consequence to be truth-preservation in all I-models in the given collection, and validity as truth in all I-models in the collection. 
what matters for the purposes of a pure semantics is not what they are or represent, but what structure is imposed on them. As such, a pure semantics can have no immediate philosophical interest as its properties are merely properties of the mathematical structures defined on the arbitrary objects with which it deals. ${ }^{119}$ A pure semantics then, while being mathematically interesting, carries no weight in philosophical debates concerning the correct model theory for explicating or defining logical consequence in various natural languages. In order to relate the semantics to that which we want it to explicate - in our case natural language - we must tell a story about what the I-models and indices within these represent. Plantinga therefore contrasts the notion of a pure semantics with that of an applied semantics. When we turn to applied semantics we are seeking a philosophical interpretation of our models which conveys a certain meaning on the terms of our formal language.

So the pure semantics as such provides a meaning for 'is a valid formula of (e.g.) S5'; it does not, as such, assign a meaning to ' $\square$ '. Nor does the pure semantics tell us what a sentence like

(6) $(\exists x) \square x$ is a person

might mean.

Logicians commonly distinguish between pure and depraved or (less censoriously) applied semantics; to get a meaning for such sentences as (6) (as well as for ' $\square$ ' itself) we must turn to an appropriate applied semantics. An

\footnotetext{
119 That is not to say that we cannot produce mathematically interesting results concerning pure semantics and the relationship between various such kinds. However, I take it that the philosophically interesting issues concerning various semantics only arise when we see the semantics as representing something or other. In this sense, the connections which might exist between various kinds of pure semantics and the properties possessed by such a pure semantics become philosophically interesting once the semantics is seen as representing something: that is, when it is no longer viewed as a pure semantics.
} 
important difference between a pure and an applied semantics is that the latter places more conditions upon the notion of modelhood. [1974: 127]

Plantinga's first point here flows from the characterization of a pure semantics as simply a collection of models consisting of various arbitrary objects with an imposed set-theoretical structure. $\square p \rightarrow p$ is a valid formula of propositional S5, but a pure semantics provides a meaning for the phrase ' $\square p \rightarrow p$ is a valid formula of S5' only in the sense that the meaning just is that $\square p \rightarrow p$ is true in all propositional S5-models. Nothing has been said about how to understand the formula itself. Plantinga goes on to cite Kripke [1963] in giving the familiar intuitive explanation of the role of the components of an I-model; $W$ is the set of possible worlds, $R$ is a relation of relative possibility, @ is the actual world, $\psi(w)$ is the set of objects existing in $w$, and so forth.

These remarks are really hints as to the intended or associated applied semantics. In the intended applied semantics, therefore, a model structure will not just be any triple $(G, K, R)$ where $G$ is a member of $K$, and $R$ is reflexive; $K$ will be a set of possible worlds (not chessmen) - possible states of affairs of a certain kind — of which $G$ is a member. And $\psi(W)$ will not just assign any domain of objects to a member $W$ of $K$; it will assign to it the set of objects existing in $W$; the set of objects, that is, that would have existed had $W$ been actual. We are to suppose, still further, that the members of $\mathcal{U}$ (i.e. the union of the domains of the possible worlds) have properties in these various worlds, or, alternatively, are such that various predicates are true of them with respect to those worlds, it being entirely proper for the same individual to have different properties in different worlds. [...] [We] get a meaning for

(6) $(\exists x) \square x$ is a person; 
what this now tells us is that in the actual world there exists an object that in every world has the property of being a person. And of course if we look at a semantics of this kind as a sober and literal account of modality - one whose talk of possible worlds and all the rest is to be taken seriously - then what is crucial is the applied, not the pure, semantics. [1974: 128]

What Plantinga is pointing out is that we need an interpretation of the various components of the models in order to convey meanings onto the sentences of our formal language. In the absence of such an explanation, the truth of a formula in a model leaves us with no clue as to what this represents. Needless to say, the same is true for formulas that are true in all models or arguments that preserve truth in all models. In order to give such a story for a modal language, Plantinga speaks as if the indices really are possible worlds. There are a number of ways in which one might try to resist the conclusion that real possible worlds - whether realist or ersatz - are needed in order to give the meaning of modal statements via a Kripke Semantics, but they will not concern us here. ${ }^{120}$ At a very minimum, we will accept that the indices, when engaged in the prospect of giving an applied semantics, in some sense or other, represent possible worlds. And it is here that the issues touched upon by Cocchiarella and Hintikka take effect.

Cocchiarella [1975] and Hintikka [1980, 1982] are not merely pointing out that Kripke Semantics, viewed as a pure semantics, delivers the wrong set of validities. In light of the above, it is unclear what this could even mean. The worry, rather, is that there is a meaning for $\square$ which a standard Kripke Semantics cannot deliver an applied semantics for, that of logical necessity. As already mentioned, the problem is supposed to be that $W$ is allowed to vary between models, whereas the proper meaning of $\square \alpha$, when $\square$ is understood as logical necessity, is given by $\alpha$ being true in all logically ${ }^{120}$ See Chihara [1998], Menzel [1990], Ray [1996b]. 
possible worlds. Since not all models are guaranteed to contain the set of all possible worlds, not all models give the correct meaning to $\square$-claims, and a standard Kripke Semantics is therefore not a viable option as an applied semantics for $\square$ understood as logical necessity. This worry, we shall argue, is not well founded from a perspective that sees the collection of Kripke Models as capturing a notion of truth preservation in all possibilities under all interpretations of the non-logical terms, as it neglects the interpretational aspect of the semantics.

\subsubsection{Etchemendy on Kripke Semantics}

Etchemendy [2008] makes two claims about what he calls index or possible world semantics. Focusing, for our purposes, on Kripke Semantics, we have the following two claims, one negative, one positive.

(neg-cl) Tarski's account of logical consequence cannot make sense of Kripke Semantics used to study consequence relations.

(pos-cl) Representational semantics provides an intuitive way to make sense of Kripke Semantics used to study consequence relations.

Now, regrettably, Etchemendy does not say much about these two in turn. The first claim is part of his overall project of showing that Tarski's account of the logical properties is fundamentally mistaken.

$[\mathrm{M}]$ uch of the most interesting work in logic during the past thirty years has grown out of the so-called "index" or "possible world" semantics, pioneered by Saul Kripke, Stig Kanger, and others. This work includes modal logic, epistemic logic, temporal logic, deontic logic, and the logic of indexicals, and so forth. Yet in none of these cases does the consequence relation studied admit of a plausible Tarskian characterization. (Etchemendy [2008: 280]) 
The second claim, though it is not elaborated fully, is the claim that we can make sense of these logics from a representational perspective. ${ }^{121}$

Recall that according to Etchemendy, Tarski's model-theoretic account endorses both (INT) and (MRA). That is, we hold fixed the actual world while we vary the interpretations of the non-logical constants, to assure that under all such interpretations the argument preserves truth. In a footnote Etchemendy comments on the application of Tarski's account to modal languages.

One might think that of all these applications, modal logic would be the one most likely to succeed. But even here, we are immediately embroiled in substantive issues expressible using the modal operators that one does not ordinarily consider part of modal logic. For example, we would have to decide (or discover) whether there are any properties which one object has necessarily, but which another object has contingently. If not, then $\forall x(\square P x \vee \square \neg P x)$ will be a (Tarskian) consequence of $\exists y \square P y$. This is not a logical consequence in any modal logic I am familiar with. [2008: 281, n.13]

Etchemendy is here applying Tarski's original definition directly to the modal language, and is not bothered with the underlying model theory. Tarski's original formulation tells us to designate a set of logical constants whose meaning we keep fixed. Etchemendy is assuming that these are the standard truth-functional connective $\vee, \neg$, the quantifiers $\forall, \exists$, and the modal operator $\square$. This is the standard choice in doing modal logics, so we can hardly blame him for this assumption. We hold these fixed and ask whether the argument

$$
\text { - } \exists y \square P y \text {. }
$$

121 These logics being modal logics, epistemic logics, temporal logics, deontic logics, the logics of indexicals, and so forth. In the present discussion we will focus on alethic modal logics, more precisely, the logics of logical necessity and logical possibility. 
- Therefore, $\forall x(\square P x \vee \square \neg P x)$.

preserves truth (in the actual world) no matter how we understand the non-logical term $P$. But, as Etchemendy notes, this depends entirely on whether there are any properties (whether expressible in our language or not) such that one object actually possesses it with necessity, but another object possesses it only contingently. If there is no such property, then under every interpretation where the premise is true the conclusion will be true as well, since all other objects will either possess this property with necessity or lack this property with necessity, no matter what property it is interpreted to be.

Now, it is clear that we can define Kripke Models in which $\exists y \square P y$ is true but $\forall x(\square P x \vee \square \neg P x)$ is false. Any model in which there is an object $o \in D_{@}$ s.t. for all $w \in W, o \in I(w)(P)$, and another object $o^{\prime} \in D_{@}$ s.t. $o^{\prime} \in I(w)(P)$ and $o^{\prime} \notin I\left(w^{\prime}\right)(P)$, for some $w, w^{\prime} \in W$, will do. Etchemendy's point is that whether such a model is acceptable from the perspective of Tarski depends on whether there actually is such a property. This, of course, depends in turn on the correct interpretation of Tarski being one such that we fix the meaning of the logical terminology and consider various objects as the denotations of the non-logical terms. If this is a correct rendering of Tarski, then whether or not this model is acceptable depends on whether there is an actual object (in this case a property), which we can take $P$ to refer to such that the premise is actually true while the conclusion is false. If there is no such property to denote by $P$, then the model in question does not represent an actual interpretation of $P$ and hence it is illegitimate. From a representational perspective, we may note, such considerations are not relevant. Models do not represent actual interpretations of the non-logical terminology, but represent various ways that the world could have been. What matters for these purposes is whether there could be a property, $P$, such that one object necessarily possesses it, but another object possesses it only contingently. If there could be such an object, then the argument is invalid. 
I will not attempt to assess whether or not Etchemendy's interpretation of Tarski's account, as it relates to modal languages, is correct or not. What we can point out, though, is that Etchemendy's choice of example is unfortunate. If we assume that for all properties, if some object possesses it with necessity, then every other object will possess it with necessity or will necessarily lack it, and that all other combinations of possessing properties contingently or with necessity are left open, we can construct other complex properties which contradict the assumption. Consider $F$ such that $a$ has it necessarily, whereas $b$ lacks it by necessity, that is, $\square F a$ and $\square \neg F b$ are true. Consider $H$ which $b$ possesses, but not in all worlds. Now, the complex property expressed by $F x \vee H x$ is such that $a$ possesses it with necessity, since $a$ possesses $F$ with necessity. But $\square \neg(F b \vee H b)$ is not true on account of the world where $b$ is $H$, and neither is $\square(F b \vee H b)$ true on account of there being a world where neither $F$ nor $H$ applies to $b$. Hence, our complex property, being $F$ or being $H$, is possessed with necessity by some object, yet it is not the case that every other object necessarily possesses it or necessarily does not possess it. Unless we also assume that genuine properties should be expressible only through simple predicates (an assumption which is not made by Tarski or Etchemendy) we see that the original assumption cannot be true, unless, of course, we make further assumptions. Assuming, for example, that if an object has a property necessarily, then every object has it necessarily, we cannot construct the above complex property. Likewise if we assume that there are no merely contingent properties. All of this is just an extension of a point made by Marcus [1967]. Marcus points out that if there are properties which in every world some objects possess while others do not, then there will be definable properties which some objects possess contingently but others necessarily. Suppose $C$ is a predicate expressing a property of the former kind and consider the predicate given by $\phi(x)={ }_{\operatorname{def}} C x \vee \neg C b$. It should be 
clear that $\square \phi(b)$, but given the assumptions about $C$, for $a \neq b$, we have $\diamond \neg \phi(a){ }^{122}$

Etchemendy is not denying that Tarskian techniques are used in developing a model theory for a modal language, as well as for an extensional language. The claim is not that such a model theory is, in all senses, strictly unrelated to Tarski and Tarski's work. The claim is merely that the notion of model-theoretic consequence, which Tarski [1956a] gives rise to, is not straightforwardly applicable to modal languages. The assumptions of (INT) and (MRA), especially the latter, do not seem to be appropriately applicable to a model theory for an intensional language.

Kripke semantics, in its many variations, bears no relation to the reductive account of logical consequence given by Tarski. To be sure, in Kripke semantics we use semantic techniques pioneered by Tarski to define the relation of truth in a structure, and we define logically true sentences to be those that come out true in every structure. But this vague similarity is as far as the resemblance goes. We conduct no investigation of which sentences involving [necessity and possibility] are actually true-that is, true [out there in the actual world]. Yet such issues would be an essential part of these investigations if Tarski's reductive analysis were correct. The answer, of course, is that in characterizing the logic of these languages we are doing something quite different. I will return to what that is later. For now, what is important to recognize is that the reductive analysis is not used in studying these languages, and would not work if it were. (Etchemendy

\footnotetext{
122 The context of Marcus' points is slightly different. Marcus is engaged in a discussion of whether a quantified modal logic commits us to essentialism. Marcus uses the example to show that the system QM is strongly essentialist, where a system is strongly essentialist if there is a property, $\psi$, s.t. it validates the sentence $\exists x \exists z(\square \psi(x) \wedge \psi(z) \wedge \neg \square \psi(z))$. $\phi(x)$, given as above, is such a property under the assumption that $\phi(a)$ is true. But, of course, there is something quite harmless about this, since it only means that in every world, the object denoted by $b$ is either $C$ or not $C$. I refer the reader to Marcus [1967] for the details of the example.
} 
[2008: 281])

Etchemendy is here saying that we are doing something quite different when we are studying these languages. Etchemendy's claim is that we are viewing the model theory from his favored modeling perspective, a perspective which is essentially representational. Unfortunately, Etchemendy does not provide any detailed explanation of how the modeling perspective, as we looked at it for extensional languages in chapter 2, extends to modal languages. Recall that from the representational perspective, we aim to give a class of models that represent all the possible ways that the world could have been which are relevant for the semantics of the terms of the language. Each model is meant to represent a possible way the world could have been, modulo the decision to treat some terms categorematically, and together the models are meant to represent all such ways. As we saw in chapter 2 , we can present evidence that a model theory for a standard propositional or first-order language manages to do this. However, as we also saw, since we are ignoring the exact semantic contribution of the terms treated categorematically, we cannot expect the models to represent all and only possible ways the world could have been relative to the semantics of all terms.

Here is how Etchemendy describes a single Kripke Model.

In a Kripke semantics, a structure consists of a set of indices, $I$, plus a relation $R$ on $I$ that specifies whether one index is "accessible" from another. Each index is associated with what is in effect a first-order structure, specifying a domain, extensions of the predicates, and so forth. The members of $I$ are for heuristic reasons called possible worlds (though they are in fact simply set theoretic objects of some sort), and one of them, sometimes denoted @, is singled out as the actual world. According to the heuristic, the first-order structure associated with a given index represents the non-modal facts of the possible world corresponding to that index. Thus the first-order 
structure assigned to the index @ represents the non-modal facts in the actual world. The remaining members of $I$, along with the accessibility relation $R$, are simply an ingenious way of representing modal facts about the world. For example, if a particular state of affairs holds at an index $i$ accessible from @, this represents that this is a possible (though perhaps not actual) state of affairs. In other words, an entire Kripke structure represents a world - a single world - replete with both modal and non-modal facts. [2008: 291]

Etchemendy is here urging that each Kripke Model represents what is true or false from the perspective of a single world, a world in which the modal facts are decided and the sentences containing modal operators are true or false accordingly. The modal facts which decide the truth values of modal sentences are represented by the indices and associated domains and valuations. These represent, relative to this one world, that the facts could have been different. The focus on just one world, what we refer to as @, serves to single out a perspective from which we can speak of each sentence of the language being either true or false. But one should keep in mind that though we are calling it 'the actual world' in all models, it is not meant to represent the actual actual world in all models, but rather, it is meant to represent the actual world as it could have been, which, of course, is just a possible world. @, therefore, serves only to mark a perspective from which to measure truth in a certain possibility, under a certain interpretation of the language. For this reason, we should expect its role to be inessential for purposes of the resulting consequence relation, something which can be verified for languages that do not contain special operators, like that of the actualityoperator, $A{ }^{123}$ If we add an operator capable of expressing what is the case at @, then it will clearly matter which index is designated as @.

\footnotetext{
${ }^{123} A \alpha$ is to be true at $w$ in $M$ if and only if $\alpha$ is true at @ in $M$.
} 
The informal notion which we are aiming to capture is the same as for extensional languages: that of being truth preserving in all possibilities in virtue of the semantics of the terms. But just as in the extensional case, when we allow for a categorematic treatment of some terms we introduce an interpretational aspect to the semantics. Treating a category of terms categorematically, as we looked at in the extensional case, amounts to not paying attention to the exact semantic nature of those terms. Instead, the formal counterparts of categormatic terms will be interpreted by a model in accordance with how at least one expression from that category could behave. For example, consider the statement ' $\mathrm{a}=\mathrm{a}$ '. This, presumably is necessary, and so should not be false. However, in a propositional language where ' $\mathrm{a}=\mathrm{a}$ ' is represented by $p$, we still have models where $\square p$ is false. This is because there are other expressions in the category of statements, say 'Barack Obama is a lizard', that can behave in that fashion. As was the case with extensional languages, this slightly complicates the picture, but for Etchemendy the crucial feature is still the following:

The crucial feature of a Kripke semantics is that for any logically possible configuration of the world, including both modal and non-modal facts, there will be a Kripke structure representing that configuration. [2008: 291]

Thus, if we follow Etchemendy's suggestions, the informal notion which we are modeling is that of being truth preserving in all possibilities in virtue of the semantics of the logical terms. (Again, I would like to remind the reader that I am using 'logical' and 'non-logical' merely as labels). We therefore get the notion of being truth preserving in all possibilities no matter what the non-logical terms are taken to mean in virtue of the semantics of the logical terms, that is, being truth preserving in all possibilities under all interpretations of the non-logical terms in virtue of the semantics of the logical terms. For ease of exposition, when referring to the notion that results, I will often drop the clause 'in virtue of the semantics of the logical terms'. 
It is important to keep in mind that the phenomenon which we are capturing, following Etchemendy, is at bottom a semantic phenomenon.

[O] ur goal in constructing a semantics is to devise a class of models that represents all logically possible ways the world might be that are relevant to the truth or falsity of sentences in the language[.] (Etchemendy [2008: 287], my emphasis)

Etchemendy is here stressing that we need to have a model of all logical possibilities as they are relevant to the truth or falsity of our sentences. This suggests that representations are to be individuated by the resulting class of true sentences. If two representations make the same sentences true, under the same interpretation, then they represent the same logically possible circumstances for the purposes of that very interpretation.

We will henceforth assume that our model theory aims to capture truth preservation in all possibilities under all interpretations of the non-logical terms. That this is what a model theory for modal languages aims to capture is explicitly endorsed by Pollock [1967], and we have already seen that Shapiro [1998] explicitly endorses this view for extensional languages. The way in which a model provides an interpretation for the non-logical constants is by assigning these different extensions relative to the different indices. This represents that the facts of the matter could have been different relative to the interpretation given to that non-logical constant. Differences in truth value between two Kripke Models are to reflect differences in the possibility represented by that model as well as differences in the interpretation of the non-logical constants.

To the extent to which it matters what the exact nature of the possibilities are, and the extent to which this is at odds with Etchemendy's view, the reader should keep in mind that we are not necessarily taking ourselves to be applying Etchemendy's notion of consequence. The minimal assumption, on which we continue, is that there are 
interpretational differences between the various Kripke Models used in our definition of validity and consequence. What this means is that each Kripke Model provides an interpretation of the non-logical terminology. When we further assume that we want to survey all possible interpretations of the non-logical terminology, we find that the approach taken in a standard Kripke Semantics — of letting the truth clause for $\square$ range over arbitrary sets of indices - is the correct approach.

Though the difference between two I-models is construed (in part) as a difference in how the non-logical terms are interpreted, it is important to note that the differences in truth value within a model, that is, at different indices, are construed as differences in how the world is represented to be. Consider the behavior of a 1-place predicate, say $F$, in the indices making up an I-model. When we consider the differences in extension between two indices we do not see ourselves as changing the meaning of the predicate $F$, but rather as reflecting the idea that $F$ could apply to different individuals than it actually (or possibly) does. Or, to put the point in another way: if our conception of the indices within an I-model is that of providing a different interpretation of the non-logical terminology, then a model-theoretic semantics for such a language would not have the bearing it has been thought to have on the interrelatedness of validities of different systems and metaphysically hard questions such as whether all instances of the Barcan Formula are true. Consider an instance of the Barcan Formula: ${ }^{124} \diamond \exists x F x \rightarrow$ $\exists x \diamond F x$. Now, if its truth in a model qua truth in the designated index were to be assessed following the above suggestion - that different indices represent different interpretations of $F$ - the truth of the Barcan Formula would express that if $F$ can be interpreted as having a non-empty extension, then there is some object which we

124 This is strictly speaking incorrect. A formula does not have instances. However, the schema $\diamond \exists x A \rightarrow \exists x \diamond A$ is often referred to as 'the Barcan Formula' even though it is a schema, not a formula. I follow suit. 
can interpret to be in the extension of $F$. But this does not seem like something anyone would deny. If there is an issue about the validity of all instances of the Barcan Formula, then the difference between indices must be a difference in how these indices represent the world to be.

\subsubsection{Logical Necessity}

We will primarily be interested in a reading of $\square$ where it is to mean logical necessity. The first thing to note about a model-theoretic semantics for a modal language where is to mean logical necessity is that it is almost universally agreed that such a semantics must work with a universal relation of accessibility. (Or, what amounts to the same thing as long as all models are considered, an equivalence relation).

The rationale behind this assumption has been expressed in different ways, but the intuitions underlying it are often the same. Consider the modal axioms of S5. ${ }^{125}$ When $\square$ is understood as logical necessity, the $\mathrm{K}$ axiom, given schematically by $\square(A \rightarrow B) \rightarrow$ $(\square A \rightarrow \square B$ ) simply says that if it is logically necessary that when the statement $A$ is true, the statement $B$ is true, then it must also be the case that if $A$ is logically necessary, $B$ must also be logically necessary. This seems intuitively right. For the T axiom, given schematically by $\square A \rightarrow A$, we may merely note that necessity, in this case logical, implies truth. For the 5 axiom, given schematically by $\diamond A \rightarrow \square \diamond A$, the following intuition is often given: ${ }^{126}$ The facts of logic, whatever they are, could not have been different. Whatever results logic produces, these are not contingent. If something is logically possible, it is possible from the perspective of every other way that the world might logically have been; hence, it is logically necessary that it is

\footnotetext{
${ }^{125}$ Complications will, and do, of course, arise when we add quantifiers to our language, but for now we are simply speaking at an intuitive level.

126 This is not to say that no rigorous arguments are ever given, but merely to say that it has become standard to accept that any modal logic where $\square$ represents 'logical necessity' must be an S5-like modality and hence be interpreted via models where the accessibility relation $R$ is either universal or an equivalence relation. Burgess [1999: 84ff] gives an argument for the assumption.
} 
logically possible, just as the 5-axiom would have it. But if $\square$, understood as logical necessity, satisfies all of the axioms of S5, then $\square$, on this understanding, must be (at least) an S5-modality. ${ }^{127}$ The frame-conditions corresponding to these axioms, it is well-known, makes it so that $R$ must be an equivalence relation. However, it is easy to see that if we work with a designated index, @, at which to define truth in a model, then the indices not in the equivalence class of @, $[w] @$, are strictly superfluous for the purpose of the one model. Even if we do not work with a designated index any model with more than two such equivalence classes can be seen, from the viewpoint of the logic, as two distinct models. Assuming, then, that whatever our models are (or represent), we must somehow have all of the ones that are not structurally isomorphic, we might as well work with a universal accessibility relation, or, what amounts to the same thing, no accessibility relation at all. ${ }^{128}$

Though we will continue to work under the assumption that the relation $R$, when logical necessity is at stake, is universal (and therefore not mention it), it is worth noting that there are those who question this assumption. Most notably, perhaps, that of Prior in his development of his modal system Q (Prior [1957: ch. V]). Prior claims that "[t]o be logically necessary a statement must not only be incapable of being false, but necessarily formulable" [1957: 50]. The idea is that it is not enough for a statement to not possibly be false, but the statement must also be statable from the perspective of all possible ways the world could have been. This is the case because, as Prior maintains, in order for a statement to be true in some possible world, $w$, the statement must be statable there, which means that the individuals that the singular terms in the

\footnotetext{
${ }^{127}$ At least an S5-modality, since this only shows that logical necessity satisfies the axioms of S5; there might be more modal axioms or rules which it satisfies as well, making it an extension of S5.

${ }^{128}$ We are working here under the assumption that our language contains only one modal operator and its dual. If our language contains more modal operators we cannot always represent models where the relation $R$, for the one modal operator, is an equivalence relation by using two or more other models. This happens, for instance, if our additional operator, call it $\square_{A}$, relates worlds in the equivalence relation of @ under $R_{\square}$ to worlds that are not in this equivalence relation.
} 
statement are about must exist at $w$. Consequently, "the statement 'It is necessary that if someone is a better logician than I am then someone is a better logician than I am' is false because there need not be any me" [1957: 50]. Prior is here pointing out that some statements which we would consider logical truths are not necessary. In particular, the formal counterpart of the formal sentence $\exists x B(x, a) \rightarrow \exists x B(x, a)$ is not necessary on account of it not being true in a possible way the world could have been, where the individual constant, $a$, fails to refer. For this reason, Prior denies that and $\diamond$ are interdefinable. While it is true that $\exists x B(x, a) \rightarrow \exists x B(x, a)$ is not possibly false, i.e., $\neg \diamond \neg(\exists x B(x, a) \rightarrow \exists x B(x, a))$, in order for it to be necessary, the statement must also be necessarily statable. Let $S \alpha$ denote that $\alpha$ is necessarily statable: that is, that the objects that $\alpha$ is about exist in all possible ways the world could have been. We can then define $\square$ by the following: $\square \alpha \leftrightarrow(S \alpha \wedge \neg \diamond \neg \alpha){ }^{129}$ If we consider it part of the semantics of $\square$, as logical necessity, that a statement should also be necessarily statable, then we get counterexamples to the 5-axiom. Consider the simple sentence 'Socrates could have existed', formalized as $\diamond \exists x(s=x) .{ }^{130}$ Ignoring temporal issues, this sentence is statable at the actual world, and furthermore it is true here, since Socrates does exist here. But there are worlds where Socrates does not exist, so it is not the case that the statement is necessarily statable, hence it is not the case that $S(\diamond \exists x(s=x))$, and consequently we do not have $\square \diamond \exists x(s=x)$. Hence, there is an instance of the 5-axiom, $\diamond A \rightarrow \square \diamond A$, which is not true. In Prior's view, the facts about what statements are logically possible or logically necessary depend in an essential way on what exists, since the truth of such statements will always depend on their statability, which in turn depends on what objects exist. Given that there could

\footnotetext{
${ }^{129}$ For this reason, Prior's system Q does not contain the rule of necessitation, i.e. $\vdash \alpha \Rightarrow \vdash \square \alpha$. Instead, Prior's system Q has a rule of weak necessitation, $\vdash \alpha \Rightarrow \vdash \neg \diamond \neg \alpha$, and a (derived) rule explicating the relationship between being necessarily statable and being necessary: $\vdash \alpha \Rightarrow \vdash(S \alpha \rightarrow$ $\square \alpha$ ). See Menzel [2008: $\S 4.2 \mathrm{ff}]$ for an explication of these points and a brief overview of Prior's views.

${ }^{130}$ I owe this example to Menzel in written communication.
} 
have existed different individuals than the ones that actually exist, the facts of logic could have been different. From the perspective of the actual world it is possible that Socrates does not exist, but for it to be true at such a world that Socrates could have existed a statement about Socrates must be true, which it cannot be on account of Socrates not existing there. Hence, there are logically possible worlds that are possible relative to the actual world, but where the actual world is not logically possible relative to such a logically possible world. We can therefore not require that the accessibility relation, $R$, is symmetric. ${ }^{131}$

Discussing the embroiled issues in the detail they demand would quite possibly require a whole new thesis, and we will therefore not pursue such a request here. Let it be noted, though, that the assumption that $R$, when deciding the truth of $\square \alpha$ for an interpretation of $\square$ as logical necessity, is an equivalence relation (or the universal relation) can be questioned. And let it further be noted that our decision not to question this assumption should not be thought of as implying that we dismiss the arguments to the contrary. Rather, our decision to treat $R$ as the universal relation comes about in order to provide a common framework from which to address the issues which are central to this thesis. ${ }^{132}$

\subsection{The Criticism of Kripke Semantics}

Kripke Semantics has been criticized on account of not being able to capture a reading of $\square$ under which it is to mean logical necessity. The two most prominent critics are

\footnotetext{
${ }^{131}$ In a similar fashion we can construct counterexamples to the 4 -axiom, $\square A \rightarrow \square \square A$, and can consequently not require that $R$ be a transitive relation (See Menzel [2008: §4.2.2.2]).

132 That is not to say that these issues are not at all relevant to our current project, but only that bringing them in at the present stage would be premature. Once we have established that there is an interesting notion of being truth preserving in all possibilities under all interpretations that is captured by Kripke Semantics, we can come back to consider these Priorean points. But such an endeavor, unfortunately, falls without the scope of the present thesis and will consequently have to wait for another occasion.
} 
Cocchiarella [1975, 2002] and Hintikka [1980, 1982], but also Hanson \& Hawthorne [1985] criticize Kripke Semantics, though their concerns are not limited to readings of as logical necessity. Our concern in the coming sections is to show that this criticism is mistaken if it is meant to apply to our current view of a valid argument of our language as one that is truth preserving in all possibilities under all interpretations.

Cocchiarella [1975] criticizes standard model theories for modal languages, exemplified most prominently by a Kripke Semantics, for incorporating a certain artifice.

[T] he artifice in question concerns allowing modal operators to range (in their semantical clauses) over arbitrary non-empty subsets of the set of all possible worlds (models) based upon the given universe of objects and the set of predicates in question. [1975: 13]

Cocchiarella is equating a possible world with a model. The models in question, though, are the models over which the truth clause for $\square$ ranges, so for a standard Kripke Semantics, these are the "models" represented by the indices with accompanying domains and interpretation functions. For this reason Cocchiarella goes on to claim that a semantics which does not allow the quantifier in the truth clause for $\square$ to range over all such models is illegitimate, at least from the perspective of a purely formal treatment of $\square .^{133}$

The intuitive and natural interpretation of modal operators for logical modalities, however, is that they range over all possible worlds (models) of a logical space (as determined by a universe of objects and a set of

\footnotetext{
${ }^{133}$ Cocchiarella does state explicitly that the troublesome "artifice introduces, at least within conceptual frameworks typified by logical atomism, a subtle form of descriptive as opposed to merely formal content into the semantics of modal operators" [1975: 13]. The criticism is therefore dependent on accepting something like logical atomism as the correct framework.
} 
predicate-nexuses) and not some arbitrary non-empty subset of the totality. The latter interpretation, by allowing the exclusion of some of the worlds (models) of a logical space, imports material conditions into the semantics of modal operators. This exclusion, however appropriate for the representation of non-logical (e.g., causal or temporal) modalities, is quite inappropriate for the representation of what are purported to be merely formal or logical modalities. [1975: 13]

The claim is thus that it is illegitimate to see a Kripke Semantics as the appropriate model theory for a language containing an operator, $\square$, for logical necessity, because the semantic clause for $\square$ is not required to range over all first-order models as they represent possible worlds.

We see the exact same thought present in Hintikka [1982].

[The standard Kripke Semantics for a modal language] is in trouble if our modal operators $N, M$ are thought of as expressing logical modalities. For there is a sense in which the truth conditions (T.N) and (T.M) are obviously inadequate. What is needed for the logical necessity of a sentence $p$ in a world $w_{0}$ is more than its truth in each one of the arbitrarily selected set of alternatives to $w_{0}$. What is needed is its truth in each logically possible world. However, in Kripke semantics it is not required that all such worlds are amongst the alternatives to a given one. Conversely, for the logical possibility of $p$ in $w_{0}$ it suffices that $p$ be true in some logically possible world. However, in Kripke semantics there is nothing to guarantee that that world is among the alternatives to $w_{0}$. Hence Kripke semantics is inadequate in its present form as a logic of logical modalities. And of course this was the primary application of modal logic historically. It is the purpose for which the Lewis systems were originally developed. Hence there is not only 
a skeleton in the closets of Kripke semanticists; the skeleton threatens to overturn the whole house.

Notice that this observation is completely independent of the relationtheoretical properties of $R$. If we require, as has been suggested, that for logical modalities the alternativeness relation is reflexive, symmetric, and transitive - i.e., an equivalence relation - then this has merely the consequence that each world $w_{0} \in S_{F}$ has as its alternatives in $F$ all the members of the same equivalence class of worlds. This does not guarantee in the least that all logically possible worlds are among the alternatives to $w_{0}$. The basic problem with the latter is that the appropriate worlds may not be in $S_{F}$ in the first place. [1982: 90-91]

First off, Hintikka is using ' $N$ ' and ' $M$ ' to denote what we use ' $\square$ ' and ' $\diamond$ ' for. The truth clauses which Hintikka is claiming are inadequate are therefore the truth clauses for and $\diamond$ in a given Kripke Model. Hintikka points out that the criticism is completely independent of $R$. The problem is not that a given world may not be related to a given other world, but that representatives of some worlds just are not there in the first place. So the criticism is not related to our decision to treat $R$ as the universal relation.

The problem, clearly articulated by Hintikka, is that $W$, for a given model, may not contain all possible worlds, and that therefore the correct meaning of $\square$ as logical necessity is not captured. But it is not entirely clear what this means. $W$ is by itself just a set and the claim that there are some worlds missing from $W$ can mean at least two things depending on how we individuate a world. As we saw above, Cocchiarella identifies possible worlds with models which can be formed from the given objects and predicates under consideration. A logical space, for Cocchiarella, is a set of objects and a set of predicates, and a possible world, relative to a logical space, is 
a combination of the given objects into extensions and anti-extensions of the various predicates. Hintikka's idea is the same.

One possible reaction here is to reply to me: Your observation does not really show that there is anything wrong with Kripke semantics. What you point out is merely the need of amplifying the usual Kripke semantics. Surely we can keep all the ingredients of Kripke semantics intact and simply add the requirement that, given $w_{0} \in S_{F}$, all the logically possible structures that can be formed from the given individuals and from the given predicates are exemplified among the alternatives of $w_{0}$ - or so it seems.

This is certainly a viable course to take technically. Nino Cocchiarella, who seems to have been the first to call attention publicly to the possibility I just mentioned, formulates the new semantics essentially by requiring that all models (in the usual first-order sense) with the same domain $D_{w_{o}}$ of individuals as $w_{0}$ occur among the alternatives to $w_{0}$.

As we already said, this is of course technically possible to do. Asking whether alethic modal logic is possible then largely becomes the question of how manageable the new logic is. [1982: 91-92]

According to both Cocchiarella and Hintikka, in the envisioned alternative to a standard Kripke Semantics - the semantics capable of capturing a meaning of $\square$ according to which it expresses logical necessity - all possible first-order structures with $\psi(w)$ as domain must be among the alternatives to $w$. This is required to evaluate $\square \alpha$, for given $\alpha$, correctly at $w$ while $\square$ retains the meaning of logical necessity. As Hintikka remarks, whether or not alethic modal logic is possible, under this assumption, is then the question of whether this logic is manageable.

Cocchiarella's alternative semantics, which he refers to as the primary semantics 
of logical necessity incorporates this assumption, though Cocchiarella does not make use of Kripke Models. Instead, Cocchiarella first defines a model to be a domain and an interpretation function. The truth of $\square \alpha$ in such a model is defined relative to all models that can be defined on the underlying domain, and a formula is a logical truth if and only if it is true in all models. ${ }^{134}$

Cocchiarella goes on to prove an incompleteness result for his notion of logical truth as long as the language contains at least one relational predicate (Cocchiarella [1975: 18]). Cocchiarella gives a rigorous proof of his result, and I should like to warn against the following intuitive, yet faulty, explanation: Suppose we could recursively enumerate the logical truths. For any non-modal $\alpha$ eventually $\square \alpha$ or $\neg \square \alpha$ will show up as a logical truth. But, if $\square \alpha$ shows up, $\alpha$ is a first-order theorem, and if $\neg \square \alpha$ shows up, it is a non-theorem of first-order logic. In effect, we have a decision procedure for first-order logic, something which we know to be impossible through Church's results.

Now, the incompleteness of Cocchiarella's alternative is certainly not unrelated to the above explanation, but it will not do in this particular case. It relies on the following two bi-conditionals:

( $\left.\mathrm{T}^{*}\right)$ For non-modal $\alpha$; $\alpha$ is a theorem of FOL if and only if $\square \alpha$ is a logical truth of the semantics.

(NT*) For non-modal $\alpha$; $\alpha$ is a non-theorem of FOL if and only if $\neg \square \alpha$ is a logical truth of the semantics.

And while the first of these is true, the latter fails. The reason is that the truth of a formula $\square \alpha$ in a model is being decided only with reference to models with the same domain. It is therefore possible for $\alpha$ not to be a theorem of first-order logic, yet $\square \alpha$ still be true in a model. This happens, for example, when $\alpha$ is a sentence true in all ${ }^{134}$ I refer the reader to Cocchiarella [1975: 15] for the exact details. 
and only domains of cardinality 2. ${ }^{135}$ Cocchiarella's semantics is not quite "strong" enough to argue directly via Church's impossibility result. Though it is not something on which we will dwell, one might find this slightly odd in the light of Cocchiarella's comments that Kripke Semantics incorporates material content into $\square$, whereas, for the requisite reading, $\square$ should be a purely formal operator. If $\square$ is purely formal, we would presumably not want $\square \alpha$ to be true in a model when $\alpha$ is not true in all logically possible worlds qua first-order models. Cocchiarella, though, defines logical necessity relative to a logical space, where a logical space, as we mentioned, is a collection of objects and predicates. Now, the point is not that Cocchiarella disguises that he defines the truth of $\square$-claims relative to such a logical space; he is quite explicit about it $([1975: 15])$. Neither is the point that such a treatment cannot be justified. I have no doubt that it can. The point is simply that such considerations do not seem purely formal either, unless one, as Cocchiarella, has already accepted something like logical atomism, where recombinations of objects qua logical spaces play a determining role.

At this point I should like to once again stress a point brought up in the introduction to this chapter. Though both Cocchiarella and Hintikka quite clearly criticize for not being able to capture the correct notion of logical necessity, there are some reasons why we might not consider our explanation below as being counter to this criticism. As already noted, Cocchiarella is explicit about criticizing Kripke Semantics relative to a background theory of logical atomism, or relative to what he calls frameworks typified by logical atomism [1975: 13]. In the same vein we may note that the concept of logical necessity, as employed by Hintikka, is presumably both theoretically and historically loaded, in a sense that might make it simply incomparable to Etchemendy's notion of logical necessity which we have looked at in previous chapters. ${ }^{136}$ The claim I

\footnotetext{
${ }^{135}$ Such as $\exists x \exists y((x \neq y) \wedge \forall z((x=z) \vee(y=z)))$.

${ }^{136}$ Hintikka is quite clearly writing in a tradition starting, more or less, with the work of C.I. Lewis,
} 
wish to make is that there is an interesting notion of logical necessity which can be captured using the approach of a standard Kripke Semantics: this is the notion of being necessarily true in virtue of the semantics of the involded terms, Etchemendy's notion of logical necessity, as we have made clear in the chapter 2 . This is what means in the object-language. The model theory captures a notion of being truth preserving in all possibilities under all interpretations, where the interpretation of the -operator is fixed in the above sense. We thus propose the following way of making sense of Kripke Semantics: a formula is valid if it is true in all possibilities under all interpretations of the non-logical constants, whereas a statement is logically true if it is true in all possibilities in virtue of the semantics of the terms. We may note that if a formula is valid in this sense, then it is also true in all possibilities under a specific interpretations, or, equivalently, it is necessarily true in virtue of the semantics of the involved terms, relative to this interpretation. It is just that the semantics of the terms treated categorematically are not needed in order for this judgment to come about. ${ }^{137}$

Whether or not we see this claim as being a counter-criticism to Cocchiarella and Hintikka's criticism of Kripke Semantics will thus depend on the historical question of whether the notion of logical necessity, which the two claim Kripke Semantics cannot capture, is even comparable to the notion of logical necessity which we are out to

whom Hintikka explicitly mentions [1982: 91] and continuing through the work of Carnap and others. For an overview of this tradition see, for example, Barcan [1946], Carnap [1946, 1947], Hintikka [1961], Kanger [1957], Lewis [1918], Lewis \& Langford [1932], Prior [1957], Quine [1947], Segerberg [1971]. Now, I am certainly not claiming that the concepts of logical necessity, as found in these authors, are all the very same concept. I am merely pointing out that the concept of logical necessity, which Hintikka claims cannot be captured by Kripke Semantics, might be intimately related to these authors and their concept of logical necessity, so as to make it unrelated to Etchemendy's concept of logical necessity, which we want to claim that Kripke Semantics can capture.

${ }^{137}$ For this reason we cannot equate the object-language meaning of $\square$ with the meta-language ascription of validity. But we may note that such a conflation, from our present approach, would be unwarranted from the very outset. Logical necessity, on this view, is a property of statements (they could not possibly be false in virtue of the semantics of the terms they contain), whereas validity is a property of formulae. And though the validity of a formula represents that the formula is logically necessary under all corresponding natural language interpretations, the concept of validity applies to different entities than the concept of logical necessity. We shall return to this point below. 
capture. Though this question is surely interesting, the remainder of the chapter will focus on explaning how we can see a Kripke Semantics as capturing our notion of logical necessity. In order to do so I find it very instructive to compare the approach taken in a standard Kripke Semantics to the approach taken in Cocchiarella and Hintikka's alternative semantics. This helps illuminate how a Kripke Semantics comes to represent what it does, but the reader should keep in mind that we are merely pointing out why, from a perspective that sees the model theory as capturing truth preservation in all possibilities under all interpretations of the non-logical constants, the proposals of Cocchiarella and Hintikka will not do. In particular, discussing the issues in relation to the criticisms of Cocchiarella and Hintikka will help us see why we cannot, within a standard Kripke Semantics, individuate a logically possible world as simply a possible valuation of the language (for propositional languages) or a first-order structure for the non-modal part of the language and decide the truth of $\square \alpha$ relative to all such worlds, something which we might think, from the very outset, ought to be the case.

Of course, if we read Hintikka's rather strong formulations, "there is not only a skeleton in the closets of Kripke semanticists; the skeleton threatens to overturn the whole house" [1982: 91], as implying that there is no interesting concept of logical necessity which Kripke Semantics captures, then what we explain below does become a counter-criticism to his criticism of Kripke Semantics.

\subsubsection{Propositional S5 Semantics}

Let us consider a standard propositional language augmented with a modal operator, $\square$, to be read as 'logical necessity'. Our language thus consists of a countably infinite set of sentence letters $p_{1}, \ldots, p_{n}, \ldots$, the usual truth-functional connectives, and, of course, a unary $\square$-operator. We refer to the set of sentence letters as $P$. The wffs of our language, $\mathcal{L}$, are given as in the extensional case (section 1.1.2), with the addition 
of the clause that if $\alpha$ is a wff of $\mathcal{L}$, then so is $\square \alpha$.

A standard Kripke Semantics for which the propositional system S5 is sound and complete can be given as follows:

(PKM) A Kripke Model is a triple $\langle W, @, I\rangle$, s.t. $W$ is a set, @ $\in W$, and $I$ is a function taking members of $W$ and returning a valuation function. ${ }^{138}$

(val-function) A valuation function is a function, $v$, s.t $v$ is a total function on $P$ into $\{0,1\}$.

We can now define the truth of a sentence of our language at an index in a model by the following recursive clauses. I use $\alpha$ and $\beta$ as variables ranging over the sentences of $\mathcal{L}$, and we write $M, w \Vdash \alpha$ to mean that $\alpha$ is true at $w$ in $M$. Let a model $M=\langle W, @, I\rangle$ be given and let $w \in W$. Then:

(s-at) $M, w \Vdash p_{n}$ iff $I(w)\left(p_{n}\right)=1$.

(s-neg) $M, w \Vdash \neg \alpha$ iff $M, w \Vdash \alpha^{139}$

(s-con) $M, w \Vdash \alpha \wedge \beta$ iff $M, w \Vdash \alpha$ and $M, w \Vdash \beta$.

(s-box) $M, w \Vdash \square \alpha$ iff for all $w^{\prime} \in W ; M, w^{\prime} \Vdash \alpha$.

We define truth in model, $M$, of a sentence $\alpha$, which we write as $M \Vdash \alpha$, in the following way.

(t-i-m) Let $M=\langle W$, @, $I\rangle$ be a model. $M \Vdash \alpha$ iff $M$, @ $\Vdash \alpha$.

We can define validity and consequence using this notion of truth in a model:

(val) A sentence of $\mathcal{L}, \alpha$ is valid iff for all models $M ; M \Vdash \alpha$.

\footnotetext{
138 I should like to remind the reader of our decision to drop mention of the accessibility relation $R$. This decision is purely an upshot of the assumption that logical necessity is a non-relative modality. ${ }^{139}$ We write ' $M, w \|{ }^{\prime}$ ' to mean that it is not the case that $M, w \Vdash \alpha$.
} 
We will sometimes write that $\alpha$ is valid in the above sense as $\models \alpha$.

(cons) A sentence, $\alpha$, of $\mathcal{L}$, follows logically from the set of sentences $\Gamma$, of $\mathcal{L}$, iff for all models $M$. s.t. $M \Vdash \gamma$ for all $\gamma \in \Gamma, M \Vdash \alpha$.

We write this as $\Gamma=\alpha$.

There are three things to note about the technical definitions provided here. First, we use a designated index @ to decide whether a sentence is true in a model. This, it turns out, is actually irrelevant to the set of validities and logical consequences of the semantics. We could just as well have defined $\alpha$ to follow logically from $\Gamma$ if and only if the argument preserves truth at all indices in all models. This point, of course, is purely technical. But since we would like truth in a model to represent truth under an interpretation of the non-logical parts of the language from the perspective of a possible way the world could have been, we prefer the former alternative. If we want truth in a model to represent truth under an interpretation from the perspective of a certain possibility, it seems that such an interpretation should make each sentence either true or false (and not both): this will be the case for all formulae of the language at @.

Secondly, this formulation of Kripke Semantics is equivalent to another formulation of Kripke Semantics which is standardly given. Instead of assigning each index a valuation function, we could instead have assigned, by a function $V$, each propositional variable, $p$, a subset of the set $W$, representing the worlds where $p$ is true. The affected clause, (s-at), would then instead read: $M, w \Vdash p_{n}$ iff $w \in V(p)$, but everything else concerning the semantics - the other truth clauses, validities, logical consequenceswould remain unaffected. I do not mean for this choice to have any philosophical implications whatsoever. Whether we give the semantics in one way or another, the idea is still that for a given formulae, $\alpha$, it is true in a model if that model interprets $\alpha$ to be true from the perspective of a possible circumstance, and it is true at an index in 
a model if the index represents a possible way the world could have been where $\alpha$ is true under the interpretation given to it by the model. This explanation, to the best of my knowledge, remains the same whether we explain $M, w \Vdash p_{n}$ via a function assigning, for each index, a value of 0 or 1 to $p_{n}$, or we explain it via a function assigning, for each propositional variable, a set of indices. ${ }^{140}$

Thirdly, it is clear that the indices play no role other than delivering a valuation function, so instead of having our I-models be Kripke Models, we could just work with sets of sets of valuation functions. This is also the way Cocchiarella [2002] presents his semantics for a propositional language augmented with an operator for logical necessity. Since an extensional model for a propositional language just is a valuation function this justifies our intuitive treatment above, where we spoke of an I-model as a collection of E-models.

Let $V$ be a set of valuation functions, and let $v \in V$. For a given $\alpha$, we write $\Vdash_{v}^{V} \alpha$ to mean that $\alpha$ is satisfied relative to $V$ and $v \in V$. We will write $\forall_{v}^{V} \alpha$ to mean that $\alpha$ is not satisfied relative to $V$ and $v \in V$.

$\left(\mathrm{s}^{\mathrm{at}} \mathrm{t}_{V}\right) \Vdash_{v}^{V} p_{n}$ iff $v\left(p_{n}\right)=1$.

$\left(\operatorname{s}^{-n e g}{ }_{V}\right) \Vdash_{v}^{V} \neg \alpha$ iff $\nvdash_{v}^{V} \alpha$.

$\left(\mathrm{s}^{\left.-\mathrm{con}_{V}\right)} \Vdash_{v}^{V} \alpha \wedge \beta\right.$ iff $\Vdash_{v}^{V} \alpha$ and $\Vdash_{v}^{V} \beta$.

$\left(\operatorname{s-box}_{V}\right) \Vdash_{v}^{V} \square \alpha$ iff for all $v^{\prime} \in V ; \Vdash_{v^{\prime}}^{V} \alpha$.

To define truth in a set of valuation functions we introduce the notion of a pointed set of valuation functions to be a pair $V=\left\langle V^{\prime}, v\right\rangle$, such that $V^{\prime}$ is a set of valuation

\footnotetext{
${ }^{140}$ If we choose the alternative formulation, the intuitive content of the claim that will concern us shortly - that the truth of $\square$-claims should be decided relative to all possible valuations of the language - becomes the claim that for any two distinct propositional variables, $p$ and $q, V(p) \cap V(q) \neq$ $\emptyset, W \backslash V(p) \cap V(q) \neq \emptyset, V(p) \cap W \backslash V(q) \neq \emptyset$, and $W \backslash V(p) \cap W \backslash V(q) \neq \emptyset$.
} 
functions and $v \in V^{\prime}$. This is done so that there is a perspective from which we can talk about a certain interpretation of the language being given.

(t-i-v) $\alpha$ is true in $V=\left\langle V^{\prime}, v\right\rangle$, and we write $\Vdash^{V} \alpha$, iff $\Vdash_{v}^{V^{\prime}} \alpha$.

The definition of logical consequence and validity remain the same.

Let us define the notion of correspondence between a Kripke Model and a pointed set of valuations, $V$.

(cor) Let $M=\langle W, @, I\rangle$. The pointed set of valuation functions $V=\left\langle V^{\prime}, v\right\rangle$ corresponds to $M$ iff $V^{\prime}=\{v: \exists w \in W: v=I(w)\}$ and $I(@)=v$.

We can prove that correspondence means making the same sentences true:

(result1) If $M$ is a Kripke Model corresponding to $V$, then for all $w \in W$, for all $\alpha$; $M, w \Vdash \alpha$ iff $\Vdash_{I(w)}^{V^{\prime}} \alpha$.

Because $V$ is defined to correspond to $M$, we can be sure that $I(w) \in V^{\prime}$, so the result is well-defined.

Proof. We proceed by induction on the complexity of $\alpha$. First, assume that $\alpha=p_{n}$ and let some $w \in W$ be given. We have the following: $M, w \Vdash p_{n}$ iff $I(w)\left(p_{n}\right)=1$ iff $\Vdash_{I(w)}^{V^{\prime}} p_{n}$. Hence the result holds. Suppose now that the result holds for all $w$ and all sentences of lower complexity than $\alpha$.

Let $\alpha=\neg \beta$, and let $w$ be arbitrary. Then $M, w \Vdash \neg \beta$ iff $M, w \Downarrow \beta$ iff (by IH) $\| \nvdash_{I(w)}^{V^{\prime}} \beta$ iff $\Vdash_{I(w)}^{V^{\prime}} \neg \beta$. The result follows in a similar straightforward manner when $\alpha=\beta \wedge \gamma$.

Suppose now, that $\alpha=\square \beta$, and let $w$ be arbitrary. Now, $M, w \Vdash \square \beta$ iff $M, w^{\prime} \Vdash \beta$ for all $w^{\prime} \in W$. But since $V^{\prime}$ contains a valuation function $I\left(w^{\prime}\right)$ for all and only $w^{\prime} \in W$, this is, by $\mathrm{IH}$, equivalent to $\Vdash_{I\left(w^{\prime}\right)}^{V^{\prime}} \beta$ for all $I\left(w^{\prime}\right) \in V^{\prime}$. And the latter is just $\Vdash_{I(w)}^{V^{\prime}} \square \beta$. 
Since this holds for all $w \in W$, it holds in particular for $I(@)=v$ and we have the following corollary:

(result2) If $M$ corresponds to $V$, then $M \Vdash \alpha$ iff $\Vdash^{V} \alpha$, for all $\alpha$.

We can see how each pointed set of valuation functions $V$ corresponds to infinitely many Kripke Models. However, for each Kripke Model there will be a unique pointed set of valuation functions that corresponds to it. Say that the unique pointed set of valuation functions which corresponds to a given Kripke Model $M$ is induced by $M$. We immediately have:

(result3) Let $M=\langle W$, @, $I\rangle$ be a Kripke Model and let $V=\left\langle V^{\prime}, v\right\rangle$ be the pointed set of valuation functions induced by that model. Then for all $\alpha, M \Vdash \alpha$ iff $\Vdash^{V} \alpha$.

It is clear that our notion of a pointed set of valuation functions have taken over the role of I-models in the intuitive explanation above. On this view, each pointed set of valuation functions $V$ provides an interpretation for the language from the perspective of a possible way the world could have been, that is, the sentences $\alpha$ s.t. $\Vdash^{V} \alpha$ are the sentences that are interpreted to be true in the possibility represented by $V$.

The difference in truth values between pointed sets of valuations therefore means that the sentences of the language are given different interpretations. In fact, things are not quite so simple. We have adopted the informal notion of a valid argument as one that preserves truth in all possibilities under all interpretations of the nonlogical terminology - as in the extensional case - so each I-model, in this case pointed set of valuations, also represents a possible circumstance. Because of this, two models disagreeing on the truth value of a sentence could represent it as having the same interpretation, but from two different perspectives. These complications are irrelevant for present purposes. What matters is that each pointed set of valuation functions provides an interpretation of the non-logical constants, in this case atomic sentences. 
Looking at the difference between the valuation functions in such a pointed set, though, the difference in the truth values assigned to the sentence letters $i s$ not to be thought of as a difference in interpretation, but that the sentence letter in question, under the very interpretation given to it by the model, could have had a different truth value because the relevant facts could have been different. That is, the valuation functions within a pointed set $V$ have taken over the role of our E-models in the intuitive explanation.

\subsubsection{There Are Valuations qua Logical Possibilities Missing}

If we are to view each pointed set of valuation functions as representing a possible way the world could have been along with an interpretation of the non-logical terms, we may naturally ask what kind of constraints we ought to put on these pointed sets.

Suppose we reason as follows:

The clause $\left(\mathrm{s}-\mathrm{box}_{V}\right)$ decides whether a sentence of the form $\square \alpha$ is true. This is to reflect the fact that $\square \alpha$ is true if and only if $\alpha$ is true in all logically possible circumstances. These circumstances are represented by the members of $V^{\prime}$. But, then, it is surely illegitimate to decide the truth of $\square \alpha$ relative to only some valuation functions; we need all of them in $V^{\prime}$ to correctly determine whether $\square \alpha$ is true.

This reasoning, I want to suggest, completely neglects the interpretational character of the pointed sets of sets of valuation functions. Each such set is meant to provide an interpretation of the non-logical terms of the language, and demanding inclusion of all possible valuation functions in each such set will exclude certain interpretations of the language from being represented.

Consider for example $V=\langle\{v\}, v\rangle$. The purpose of having this pointed set of valuation functions in determining validities and logical consequences just is to have 
an interpretation of the sentence letters in which each sentence letter is either logically true (in the sense of being logically necessary) or logically false (in the sense of not being logically possible). If this is a legitimate interpretation, then the above reasoning cannot be correct. If we demand that all possible valuation functions are present in all pointed sets of valuation functions, we will not have an interpretation of any given $p$ (from the perspective of a possible way the world could have been) such that $\square p$ is true.

There is an alternative to Kripke Semantics as a model theory explicating the semantics of a propositional language containing $\square$ to be read as 'logical necessity'. This alternative is developed in some detail in Cocchiarella [2002]. The alternative differs from a semantics explicated via sets of valuation functions as we have presented above, and have seen to be equivalent to a standard Kripke Semantics, in that we now work with all possible valuation functions in our truth clause for sentences of the form $\square \alpha$.

Let $T$ be the set of all valuation functions. We define truth at a valuation function, $v \in T$, in the exact same manner as before, but of course, the clause for $\square$ will now range over all valuation functions. That is, while the truth of sentences having truthfunctional operators as their main connective are still decided locally in the above fashion, the clause for $\square \alpha$ is now relative to every valuation function, since every valuation function is per definition in the set $T$. With the obvious changes in notation we have:

(s-a-box) $\Vdash_{v}^{T} \square \alpha$ iff for all $v^{\prime} \in T ; \Vdash_{v^{\prime}}^{T} \alpha$.

We say that a sentence of our language is valid $_{m}$ iff it is true at all valuation functions in $T$;

(a-val) A sentence, $\alpha$, of $\mathcal{L}$ is valid $_{m}$ and we write $\models_{m} \alpha$ iff for all $v \in T$; $\Vdash_{v}^{T} \alpha$. 
Similarly, we can define logical consequence in the straightforward way as being truthpreservation at all valuation functions in $T$.

The first thing to note, if we are seeking to bring this into line with our above conception of such a semantics, is that there are no two distinct I-models seen as pointed sets of valuation functions involved in the definition of validity, there is only the one set of valuation functions, $T$, consisting of all valuation functions.

We can, however, recover a notion of distinct pointed sets of valuation functions from this definition of validity and logical consequence. A pointed* set of valuation functions, on this view, is simply a pointed set of valuation functions, $V=\left\langle V^{\prime}, v\right\rangle$ with the added restriction that $V^{\prime}$ is the set of all valuation functions, $T$. What we have defined as truth at a valuation function, $v$, above then becomes truth in a model for the pointed* set of all valuation functions $\langle T, v\rangle$. It is easy to see that defining validity (or logical consequence) as truth (or truth-preservation) in every pointed* set of valuation functions so conceived leaves the notion of validity (and logical consequence) unchanged. However, we can now equate truth in a pointed* set of valuation functions with truth under an interpretation and ask what kind of interpretation each pointed* set of valuation functions provides.

Cocchiarella, as we shall come back to, is motivated by a logical atomist framework. We may note how this is reflected in his semantics. Since all possible valuations are present in the pointed* sets of valuation functions, there will always be valuation functions such that $p$ and $q$ are both true, both false, or one is true while the other is false, for any two atomic sentences. Hence, no pointed* set of valuation functions can represent there being any dependencies (in terms of $\square$ ) between two atomic sentences.

Burgess [2003] criticizes the resulting notion of validity for working with a nonstandard understanding of the sentence letters, $p_{1}, p_{2}$, and so on. In fact, Burgess 
argues for a stronger conclusion: that Kripke Semantics is an adequate semantics for a propositional language augmented with a logical necessity operator exactly because the variation in indices (and so, also in valuation functions) between Kripke Models is strictly needed to provide a standard treatment of the sentence letters.

To see what Burgess has in mind, let us try to follow his argument from the start. Burgess opens with the age old distinction between use and mention.

$[\mathrm{O}]$ ne often finds the letters $p, q, r$, and so on, employed in two quite different ways, exemplified by the following:

(X) If $p$ is any statement, then the negation of $p$ is another statement, one which is true if $p$ is false and false if $p$ is true, and logically necessary if $p$ is not logically possible, and logically possible if $p$ is not logically necessary.

(Y) The formula $p \vee \neg p$ is valid because on any valuation (that is, any assignment of values $\top$ and $\perp$ to statement letters $p, q, r \ldots)$ the formula receives the value $T$. The formula $p \& \neg p$ is unsatisfiable because there is no valuation on which the formula receives the value $T$.

What is the difference here? In $(\mathrm{X}), p$ is being used to refer to statements such as "Snow is white" or "Grass is green", and more specifically is being used as variable ranging over such statements. In (Y), $p$ and $q$ and $r$ are being mentioned as components out of which formulas are built up with the help of the logical symbols such as $\neg$ and \& and $\vee$. It would represent a confusion between use and mention, or if you will, between metalanguage and object language, to speak of formulas as true or false, or as logically necessary or logically possible — or inversely, to speak of statements as assigned value $\top$ or assigned value $\perp$, or as valid or satisfiable. [2003: 146] 
The point is that sentences of the formal language are not the kind of thing that can be true or false: sentences of the formal language are true or false in models, and the goal of having a semantics is to link this notion of truth in a model to the notion of truth or falsity of actual statements. Our definition of validity and logical consequence in the formal language are given in purely mathematical terms. Only insofar as we explain and motivate a link between these mathematical structures and the natural language phenomenon we are interested in can we begin to discuss the link between notions like validity, as applicable to formal sentences, and logical truth as applicable to actual statements.

Burgess suggests that a formal sentence is to be valid (that is, true in all models) just when replacing each sentence letter with a statement the result is true. It is possible to have different notions of what it means for a statement to replace a sentence letter $p_{n}$ in a formal sentence. Burgess [2003: 147-148] distinguishes between an instantiation of a formal sentence and an instantiation* of a formal sentence.

(ins) An instantiation of a formal sentence $A$ is the result of replacing the sentence letters in $A$ uniformly with statements to obtain a natural language sentence (with simultaneous replacement of 'and', 'or', 'not', and so on for the logical constants). Thus, 'Snow is white or snow is not white' is an instantiation of the formal sentence ' $p \vee \neg p$ ', and it is also an instantiation of the formal sentence ' $p \vee q$ '. Instantiations are the kind of thing that can be true or false, or logically necessary, or logically possible.

Contrast this with the notion of a substitution in $A$.

(sub) A substitution in the formal sentence $A$ is the result of replacing each sentence letter in $A$ with a sentence of the formal language. Thus, ' $(p \wedge \neg p) \vee(r \rightarrow q)$ ' is a substitution in ' $p \vee q$ ', but not in ' $p \vee \neg p$ '. Substitutions are the kind of thing 
that can be true in a specific model, true in no models, or perhaps true in all models.

Burgess contrasts the notion of an instantiation with the notion of an instantiation*.

(ins*) An instantiation* of the formal sentence $A$ is the result of replacing each sentence letter in $A$ uniformly with statements that are logically atomic to obtain a natural language sentence (with simultaneous replacement of 'and', 'or', 'not', and so on for the logical constants). ${ }^{141}$ Hence, 'Snow is white or snow is not white' is an instantiation* of ' $p \vee \neg p$ ', but it is not an instantiation* of ' $p \vee q$ ', since the statement 'snow is not white' contains a term, 'not', the formal counterpart of which is currently treated as a logical constant and thus is not logically atomic.

Now, according to Burgess, and I certainly think this is right, we are interested in more than truth in all instantiations* when we describe a formal sentence as a validity.

The standard aim of logicians at least from Russell onwards has been to characterize the class of formulas all of whose instantiations are true. Thus, though Russell was a logical atomist, when he endorses $p \vee \neg p$ as a law of logic, he did not mean to be committing himself only to the view that the disjunction of any logically atomic statement with its negation is true, but rather to be committing himself to the view that the disjunction of any statement whatsoever with its negation is true[.] [2003: 147]

Burgess is here claiming that a validity is standardly seen as a formal sentence all of whose instantiations are true, not merely one all of whose instantiations* are true. We aim to be as general as possible when doing logic, and hence when circumscribing the set of validities.

\footnotetext{
141 Burgess qualifies in parentheses that if not literally logically atomic, then at least without further structure that can be represented using the terms currently treated as logical constants [2003: 148].
} 
Burgess goes on to claim that only Kripke Semantics and not the envisioned alternative allows us to see a validity as a formal sentence which is true in all instantiations. The alternative, Burgess claims, provides the notion of a validity as a formal sentence all of whose instantiations* are true. The further claim, from there, is of course that the former notion of a valid sentence is our standard notion. This is what leaves Burgess to conclude that the alternative is simply working with a non-standard understanding of the role of the sentence letters in a formal language. On our standard understanding, these are properly used as variables ranging over all statements, whereas on the nonstandard understanding they are used as variables ranging over only logically atomic statements.

Using the terminology of the present thesis, what Burgess is pointing out here is that if we accept that each pointed set of valuation functions should contain all possible valuation functions, then we neglect to pay attention to the interpretational character of the semantics. A validity is a sentence which is true under all interpretations of the nonlogical terminology, and truth under all interpretations is truth in all instantiations, not in all instantiations*. The different I-models represent different interpretations of the non-logical terms, and as we have seen, demanding that the I-models consists of all possible valuations results in excluding certain interpretations. By realizing that the differences between two I-models are (in part) interpretational differences, we see that we cannot impose that each pointed set of valuation functions should consist of all possible valuation functions.

To convince ourselves that there really is a difference here, we may reflect on the fact that on the semantic alternative, validities (and so logical consequences) are not closed under uniform substitution, which they are on our Kripke style alternative. Since it is easy to see that an instantiation of a substitution, $\beta$, in $\alpha$ is also an instantiation of the original $\alpha$, the fact that validity ${ }_{m}$ is not closed under uniform substitution immediately 
rules out the fact that a valid $\mathrm{v}_{m}$ sentence is true in all instantiations. Consider the valid $\mathrm{d}_{m}$

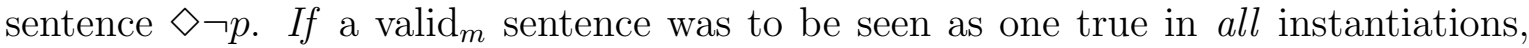
then this should not be a validity, since there are surely instantiations making it false. Any instantiation of $\diamond \neg(p \rightarrow p)$ is also an instantiation of $\diamond \neg p$, and so, since $p \rightarrow p$ is a validity, all of its instantiations must be true, and hence, it is not logically possible for it to be false. The same point could be made by pointing out that 'It is logically possible that it is not the case that if it is snowing, it is snowing' is an instantiation of $\diamond \neg p$, but the former certainly seems false. Of course, the former is not an instantiation* of $\diamond \neg p$, since 'If it is snowing, then it is snowing' contains an expression, 'if... then...', the formal counterpart of which we are currently treating as a logical constant.

At this point we may recall our definition of a reading in the previous chapter. What we are currently pressing is that all acceptable readings are to be legitimate instantiations of the sentence letters of a valid formal sentence. We said then that 'Jones is happy and Smith will get the job' is an acceptable reading of $p . \square(p \vee \neg p)$ is valid in both types of semantics, but only in the Kripke style semantics does this license us to the claim that 'It is logically necessary that either Jones is happy and Smith will get the job or it is not the case that Jones is happy and Smith will get the job' is a logical truth. As with the Russell example above, it is natural to assume that by endorsing $\square(p \vee \neg p)$ as a validity we mean to endorse all acceptable readings of $\square(p \vee \neg p)$ as logical truth, or in terms of instantiations: that all instantiations of $\square(p \vee \neg p)$ are logically true. This is the standard understanding of what $p$ represents. Note, incidentally, that this is not to say that the semantic alternative cannot explain the logical truth of 'It is logically necessary that either Jones is happy and Smith will get the job or it is not the case that Jones is happy and Smith will get the job'. However, because of the non-standard understanding of what $p$ and $q$ represent, this will be explained through the validity of $\square((p \wedge q) \vee \neg(p \wedge q))$. The validity of the 
former, $\square(p \vee \neg p)$, in relation to the natural language sentence is irrelevant.

We may also point out that there are statements that presumably are logically necessary, even though they do not exhibit a logically complex form in accordance with the current logical constants, for example, the statement 'All horses are horses'. If we agree that this statement can be used as an instantiation* of $\diamond \neg p$, then there is a valid $_{m}$ sentence which has a false instantiation* and it follows that truth in all models does not even capture truth in all instantiations*.

It is natural to imagine a proponent of validity ${ }_{m}$ stressing that when we consider such statements as 'All horses are horses' we must at the same time expand our formal language to include the formal counterparts of 'All', the universal quantifier $\forall$. I think this reply goes a long way, but it also takes much of the explanatory power away from propositional modal logic. What a propositional modal logic ought to be able to explain (or perhaps predict) is that certain sentences involving the modal terms under consideration will be true if certain other sentences are. Consider for example, the $\operatorname{valid}_{m}$ argument $\{\square p\} \models{ }_{m} \neg \square p$. This is valid $_{m}$ since there simply are no pointed* sets of valuation functions in which $\square p$ is true. Yet there are plenty of instantiations which make the premise true and the conclusion false. Consider the argument:

- It is logically necessary that all horses are horses.

- Therefore, it is not logically necessary that all horses are horses.

It seems to me that anyone who understands the content of the premise can see that it is true, while the conclusion is straightforwardly false. That is, defining as admissible instantiations* statements which do not exhibit any logical form the formal counterpart of which is currently under investigation, the argument is not even truth-preserving in all instantiations*, yet, it is a fact that $\{\square p\} \models_{m} \neg \square p$. At this point I should like to stress that we are not claiming that the alternative semantics gives no explanation as 
to why this argument is valid: allowing only logically atomic statements the premise, $\square p$, is contradictory, and so what we have here is in a certain sense just explosion. Everything follows from $\square p$ because it is contradictory given the restrictions on what $p$ represents. So, the concern here is not that the present semantics cannot explain why we have $\{\square p\} \models \models_{m} \neg \square p$, it is that it cannot explain the relation between this formal result and the above simple natural language argument.

The proponent of $\models_{m}$ would at this stage, I believe, reply that what this really shows is that 'All horses are horses' is not logically atomic simpliciter. Cocchiarella [1975, 2002] is quite explicit in his endorsement of a logical atomist framework, and from the perspective of logical atomism, it seems quite reasonable to assume that 'All horses are horses' is not logically atomic. Cocchiarella may therefore respond that his envisioned alternative is a model of truth preservation under all instantiations*, where the instantiations* truly are logically atomic simpliciter.

Now, I do not want to argue against this suggestion, as it is clearly a viable position to hold. But it does not seem to provide us with an absolute standpoint from which there is something wrong with our Kripke style alternative. By allowing all possible pointed sets of valuation functions, rather than only all possible pointed* sets of valuation functions, we are thereby representing all possible interpretations of the non-logical terminology (from the perspective of all possible ways the world could have been). Letting the sets of valuation functions within each pointed set vary arbitrarily secures that all relevant interpretations are present. This does not mean that we are incorporating material content into the semantics of $\square$, as Cocchiarella claims it does [1975: 13]. Rather, from the perspective of capturing a notion of a valid argument being truth preserving in all possibilities under all intepretations, what this shows is that the present framework does not rest on a once and for all principled distinction between being logically atomic and being logically complex. These two notions, in the 
end, come down to the notion of the logical form of the language in question. The present framework is therefore neutral on the question of logical form, but remaining neutral on the question of logical form does not seem to be incorporating material content, unless, of course, one already accepts something like logical atomism as the true theory of the world.

Of course, Cocchiarella's claim is modulo accepting a logical atomist framework, so in that sense he is right. If we accept a theory of logical atomism, then we could naturally want our semantics for the language to reflect the commitments to logical form which follow from this theory - and this claim could be accepted even from our present perspective too. But, unlike the case where we simply choose to treat some terms as logical, these commitments are given by a metaphysical, or ontological, theory of the world. We can see this clearly when we reflect on what it takes to be logically atomic. The answer cannot be given by the semantics as this relies on a certain relationship between natural language and formal language, which in turn, relies on the notion of being logically atomic. $\diamond p$ tells us that this is true for all logically atomic simpliciter statements $p$, but it does not tell us what being logically atomic consists in.

By accepting a framework in which truth preservation in all models mirrors the notion of truth preservation in all possibilities no matter what logically atomic expression the non-logical terms are interpreted as, we severely limit the explanatory power of our theory of consequence. This point extends beyond modal languages. The extensional validity of an argument in modus ponens form explains its validity for all instantiations of its parts, or so it might at least be assumed. Consider for example:

$\left(\mathrm{P}_{1}\right)$ If Napoleon has more properties than Aristotle, then there is a property which Napoleon has but Aristotle lacks. 
$\left(\mathrm{P}_{2}\right)$ Napoleon has more properties than Aristotle.

(C) There is a property which Napoleon has but Aristotle lacks.

If we were to follow the line of reasoning above, and take the formal logical consequences to mirror only truth in all logically atomic simpliciter instantiations*, then we should equally exclude 'Napoleon has more properties than Aristotle' and 'There is a property which Napoleon has but Aristotle lacks' as instantiations* of $p$ and $q$ in the argument from $p \rightarrow q$ and $p$ to $q$, since these exhibit a logical form that is not atomic. As an upshot, propositional logic cannot explain the validity of the argument from $\left(\mathrm{P}_{1}\right)$ and $\left(\mathrm{P}_{2}\right)$ to $(\mathrm{C})$, it cannot explain why this is an instance of logical consequence. This, I maintain, does not seem right. Presumably, a correct formalization would belong to higher order logic and be something like the following: ${ }^{142}$

$\left(\mathrm{f}^{-} \mathrm{P}_{1}\right) \quad((\forall X: X n \leftrightarrow X \in \Phi) \wedge(\forall Y: Y a \leftrightarrow Y \in \Psi) \wedge(|\Phi|>|\Psi|)) \rightarrow(\exists Z: Z n \wedge \neg Z a)$.

$\left(\mathrm{f}-\mathrm{P}_{2}\right)(\forall X: X n \leftrightarrow X \in \Phi) \wedge(\forall Y: Y a \leftrightarrow Y \in \Psi) \wedge(|\Phi|>|\Psi|)$.

(f-C) $\exists Z: Z n \wedge \neg Z a$.

And of course this is a valid argument of such a logic. In fact, we need not even check all of the models, and that we do not need to do so is exactly the point: We can reason meta-logically by considering the truth-functional behavior of $\rightarrow$ in all models: pick any model in which $\left(\mathrm{f}-\mathrm{P}_{1}\right)$ and $\left(\mathrm{f}-\mathrm{P}_{2}\right)$ are both true, then by the truth clause for $\rightarrow$ (f-C) must be true in that model as well. This explanation is the same, and so would have worked just as well, were we concerned with a simple propositional language. The explanation does not become better or worse whether the constituent expressions are logically atomic simpliciter.

\footnotetext{
142 Note that we are taking the natural language expression 'has more properties' to mean has strictly more properties. I think this adheres well with common usage. If the reader disagrees, though, the point remains by the appropriate substitution in the argument from $\left(\mathrm{P}_{1}\right)$ and $\left(\mathrm{P}_{2}\right)$ to $(\mathrm{C})$.
} 
The point here is not that the argument in question is deemed invalid when its proper logical form is exposed, since it clearly is valid. The point is that even if we assume that there is such a thing as the logical form, the validity of this argument is independent of its exact logical form. We presumably want it to be sufficient to point to the validity of a propositional modus ponens argument, but this is exactly what we cannot do if we are not allowed to treat 'Napoleon has more properties than Aristotle' as an interpretation of a propositional variable $p$.

\subsubsection{There Is A Fixed Class of Logical Possibilities}

As we have seen, we cannot demand that each pointed set of valuation functions contain all possible valuation functions, if we are to capture a phenomenon of being truth preserving under all interpretations. Each pointed set $V$ gives us an interpretation of the language and even though the valuation functions in this set are to be viewed representationally (i.e., as representing that a given sentence could have had a different truth value), it is illegitimate to claim that all possible valuation functions should be in the range of the quantifier of $\left(\mathrm{s}-\mathrm{box}_{V}\right)$ within such a pointed set, $V$. In the most basic case this is reflected in the fact that if $p_{i}$ is being interpreted to mean something that is logically necessary, then there should not be a valuation function, representing a possible way the world could have been, where $p_{i}$ is false. This would, as it were, change the interpretation of $p_{i}$ to something such that it is not logically necessary, since we are now representing that the facts could have been such that $p_{i}$ was false.

We have made this point using pointed sets of valuation functions, but we can easily extend these points to Kripke Models. The envisioned alternative of working only with pointed* sets of valuation claims that all possible valuation function should be in the set, whereas for Kripke Models it becomes the following claim:

- Let $M=\langle W, @, I\rangle$ be a Kripke Model. For each valuation function, $v$, there is 
$w \in W$ s.t. $I(w)=v$.

If we define validity by reference to only such models, we will be excluding the requisite interpretations of the non-logical terminology. Each Kripke Model for which all possible valuation functions are attached to an index will induce a pointed set of valuation functions, $V=\left\langle V^{\prime}, v\right\rangle$, where the assumption now assures that $V^{\prime}$ is $T$, the set of all valuation functions. That is, the Kripke Models specified as above will induce all and only pointed* sets of valuation functions. And since each Kripke Model makes the exact same sentences true as the pointed set of valuation functions it induces, it follows that there can be no interpretation of the sentence letters in accordance with which they are logically necessary or depend logically for their truth upon the interpretation of other sentence letters. The resulting validities and logical consequences are therefore the same as those given by the alternative which we denoted ' $\models{ }_{m}$ ' above. Thus, the exact same points that we have already discussed apply when explicating the semantics via Kripke Models rather than pointed sets of valuation functions: each model provides an interpretation of the language in accordance with which each sentence is either true or false. Including indices with valuation functions not already in a given Kripke Model will typically change the interpretation the sentences. This, then, undermines the reason for having that very Kripke Model in the first place. Excluding Kripke Models where not every possible valuation function is attached to an index is, in effect, tantamount to working with a non-standard treatment of the sentence letters. Under such a treatment a validity is a formal sentence true in all instantiations*, and as we saw, such a treatment severely limits the explanatory power of our model-theoretic characterization of logical consequence.

Notice, incidentally, that these points are completely independent of the modality present in our informal notion of consequence: that of being truth preserving in all possibilities under all interpretations. All we have relied on in arguing that the valu- 
tion functions within a pointed set of valuation functions should be allowed to vary arbitrarily, and consequently that the valuations attached to the indices in a standard Kripke Semantics for a propositional language should also be allowed to vary arbitrarily, is that we want a representation of each interpretation of the language.

However, since it is admitted that the indices in a Kripke Model are to be viewed representationally, one might start to wonder whether there should be a certain set of indices available in all models, after all. Here the difference between Kripke Models and simple pointed sets of valuation functions becomes crucial. The discussion so far has focused on an equivalent presentation of the model theory using sets of valuation functions as components of I-models. In such a presentation the distinction between frames and models in Kripke Semantics cannot be made. When we turn to Kripke Semantics we can choose to view a world as simply an index, a member of $W$, or as an index $w$ along with $I(w)$. A claim to the effect that there are worlds missing in some Kripke Models, then, is perhaps not about the interpretations which a collection of Kripke Models give rise to, but should perhaps rather be construed as the claim that the frame on which our Kripke Models are built should reflect something about the "modal reality" which we are currently studying - in this case, of course, the space of logical possibilities. ${ }^{143}$

On this view, then, what matters is not which valuation functions are attached to what indices, but that there is a certain number of indices. The cardinality of $W$ should remain stable across models. A Kripke Frame, $F$, is a tuple $\langle W$, @ $\rangle$, where $W$ is a set and @ $\in W .{ }^{144}$ What this really amounts to is demanding that validities be

\footnotetext{
${ }^{143}$ We will later look at a claim to this effect made by Hanson \& Hawthorne [1985], a claim that validity for a modal language should be decided on just one frame: the intended frame which reflects the structure of the modal space under investigation. However, this discussion is best taken in relation to quantified languages since those are Hanson \& Hawthorne's primary object of investigation.

${ }^{144}$ Of course, the standard definition of a Kripke Frame mentions an accessibility relation, $R$, on the members of $W$. We have already given our reasons for considering $R$ to be the universal relation, and
} 
decided on the same Kripke Frame, the frame where $W$ reflects the cardinality of the modal space of interest. However, it is easy to see that if we do not put restrictions on the valuation functions which attach to the indices, the class of validities and logical consequences will not be affected.

(result4) Let $M=\langle W, @, I\rangle$ be a Kripke Model. Define another Kripke Model as: $M^{\prime}=$ $\left\langle W \cup\left\{w^{\prime}\right\}\right.$, @, $\left.I^{\prime}\right\rangle$, such that $I^{\prime}(w)=I(w)$ for all $w \in W$ and $I^{\prime}\left(w^{\prime}\right)=I\left(w^{\prime \prime}\right)$, for some $w^{\prime \prime} \in W$. Then for all $\alpha ; M \Vdash \alpha$ iff $M^{\prime} \Vdash \alpha$.

Proof. Follows from previous results, since both these Kripke Models will clearly induce the same set $V$

What this shows is that when there are no restrictions on $I$-when we are allowed two or more indices with the same valuation function - there will be no change in the output of the semantics. If we allow all models built on just one frame, then under certain reasonable conditions, i.e., that $|W| \geq 2^{|P|}$, the collection of Kripke Models will induce exactly all pointed sets of valuation functions. The set $V=\langle\{v\}, v\rangle$, for example, will be induced by all models, $M=\langle W$, @, $I\rangle$, s.t. $I(w)=v$, for all $w \in W$, and so forth. In short, if we see a Kripke Model as representing an interpretation of the language in accordance with which each sentence is true or false, then all the same interpretations will be available for the standard approach and for the approach where validity is defined as frame-validity on $F=\langle W$, @ $\rangle$, where $|W| \geq 2^{P}$. If we maintain that there are indices missing in a standard Kripke Semantics, therefore, it cannot be about what interpretations should be available for the language, unless interpretations are understood as being determined by something more than a truth value. For a propositional language it is hard to see what this extra aspect could be, but this situation, of course, could change when we turn to quantified languages. We so dropping mention of it altogether. 
will come back to these issues later.

Adding indices with valuation functions already present will not have an effect on the truth values of the sentences. In other words, they will not be representationally relevant for a given interpretation provided by a Kripke Model. Let us say that two valuation functions, $I(w)$ and $I\left(w^{\prime}\right)$, within a Kripke Model are representationally equivalent in the model if, and only if, the truth values of the sentences in the model do not change when we remove one of either $I(w)$ or $I\left(w^{\prime}\right)$. What this then means is that the information represented by one of these valuation functions is superfluous in relation to the possibility and interpretation represented by that model. If our only interest in the representational capacities of the models is that they should represent facts that are relevant to the truth or falsity of the sentences of the language, we can see how there are no essential differences between the two.

\subsection{Quantified Languages}

As we have seen, Cocchiarella and Hintikka both criticize Kripke Semantics on the grounds that not all logically possible worlds are required to be in a given model. The claim that some models do not contain all logically possible worlds can be understood in one of two ways. Consider a Kripke Model for a standard first-order language, $M=\langle W, @, \psi, D, I\rangle$. The truth clause for $\square \alpha$, for a formula $\alpha$, is given recursively at $w \in W$ as: ${ }^{145}$

$$
M, w \Vdash \square \alpha \text { if and only if for all } w^{\prime} \in W: M, w^{\prime} \Vdash \alpha \text {. }
$$

As we saw Hintikka claim, this is only legitimate as an account of $\square$ as logical necessity if $W$ contains all logically possible worlds. But depending on how we individuate a logically possible world, this can mean different things. We can understand a world

\footnotetext{
${ }^{145}$ Note that this is just the familiar truth clause, $M, w \Vdash \square \alpha$ iff for all $w^{\prime}$ s.t. $\left\langle w, w^{\prime}\right\rangle: M, w^{\prime} \Vdash \alpha$, with $R=W^{2}$.
} 
as simply a member of $W$, in which case the criticism becomes that $W$ should have a certain size in each model, a size that reflects how many logically possible worlds there really are, and similarly, constraints should be put on the function $\psi$ in order to adequately capture the number of objects existing in those worlds. ${ }^{146}$ We will look at a claim from Hanson \& Hawthorne [1985] to this effect in a later section.

On the other hand, we can understand a world as being individuated by "what goes on there", in the sense that the properties of $\psi(w)$ and $I(w)$ both help to determine what logical possible world is represented by a given $w \in W$. This latter way of understanding the claim is what Cocchiarella [1975] and Hintikka [1982] intend, as they both develop alternative semantics where a world is equated with a first-order model. The way in which a model contains a representative of each logically possible world is therefore to have the truth clause for $\square$ range over all first-order structures definable on $\psi(@)$ for the non-modal part of the language.

Let us now see, whether from our present modeling perspective, we should endorse this claim. In terms of Kripke Models, the only legitimate models are the following:

(AFP) Let $M=\langle W$, @, $\psi, D, I\rangle$. Let $\psi(@)=D$. For every possible first-order structure for the non-modal part of the language defined on $D,\left\langle D, I_{E}\right\rangle$, there must be $w \in W$ s.t. $\psi(w)=D$ and $V(w)=I_{E}$.

Of course, there may be $w \in W$ such that $\psi(w) \neq \psi(@)$ and consequently, to give the correct truth clause for $\square$-claims at this $w$, we would have to require that all first-order structures definable on $\psi(w)$ be present in the model. We will argue that (AFP) is an unreasonable condition to put on the models because no model meeting this criteria will be able to deliver certain interpretations for the language. Since a model containing

\footnotetext{
146 'Really are' is not meant to convey a commitment to possible worlds as real existent entities in the sense of Lewis' modal realism (Lewis [1986]). What we mean by the above is just that whatever logically possible worlds are, there must be a certain number of them in order for some models not to contain them all.
} 
additional indices to those required by (AFP) will also meet the criteria, the result carries over to such models. We note the following fact:

(fact) On the (AFP)-approach formulae of the form $\diamond \neg \mathcal{F} \eta$, where $\mathcal{F}$ is a 1-place predicate and $\eta$ is an invidual constant, are valid.

It should be relatively clear why this is the case. If we suppose that there is a formula, say $\diamond \neg F a$, which is false in a model, it follows that there can be no index, $w$, in that model such that $I(w)(a) \notin I(w)(F)$, and this latter contradicts the assumption given in $(\mathrm{AFP})$.

We may also note how (AFP) can be motivated from the perspective of logical atomism, something which is explicitly endorsed by Cocchiarella [1975: 13]. A logically possible world, from the perspective of logical atomism, is simply a recombination of the objects that exist. This is why all first-order structures definable on $\psi(@)$ are to be possible relative to @, as these possible worlds are simply recombination of the existing objects into extensions and anti-extensions for various predicates. For this reason it is easy to see that from the perspective of the designated world @, there will be no non-trivial dependencies between the non-logical terminology of the language with respect to the box-operator. If we see our atomic formulae as reflecting the obtaining or non-obtaining of atomic facts, by which we mean that a certain atomic formula is true if the corresponding atomic fact obtains, we see how this is well motivated from a logical atomist perspective; a model, or world, on this approach is simply an indication of which atomic facts obtain and which do not, just as logical atomism would have it.

The validity of a formula is meant to capture that it is true in all possibilities under all interpretations of the non-logical terminology. Consider the formula $\diamond \neg F a$ and an interpretation of $F$ as the complex predicate given in English by "is green or not 
green' and $a$ as the name of any object, say, The Eiffel Tower. ${ }^{147}$ This gives us an interpretation of the formal sentence, $\diamond \neg F a$, according to which it expresses what the English statement 'It is logically possible that The Eiffel Tower is not either green or not green' expresses, which is presumably false in all possibilities. We can therefore not maintain that our model-theoretic notion of a validity captures a notion of being true in all possibilities under all interpretations. ${ }^{148}$ The reason is that we now have an interpretation of $\mathrm{Fa}$ which is not represented by any model, since a model compatible with this interpretation would have to have $F a$ true at all indices, since The Eiffel Tower is either green or not green' is true in all logically possible worlds.

Now, the proponent of the (AFP)-approach will most likely respond to the latter example by pointing out that logical necessity is a formal concept. When it applies, it applies solely in virtue of the form of the statement to which it applies. ${ }^{149}$. By pointing out that $F a$ is not true in virtue of logical form alone, we see that $\square q u a$ being true in virtue of logical form alone should not truly apply to Fa. Consequently, there should be no interpretations under which $\square F a$ is true, and its negations is consequently true under all interpretations. But at this point we may want to remind ourselves about the point we also saw Burgess make in a previous section [2003: 146]: Formulae, and so also $F a$, are not the kind of thing that can be true or false, let alone in virtue of form. Fa, like other formulae, can be true or false in models, conveying that if a formula is true in a model, then it is given an interpretation such that it is true from the perspective

\footnotetext{
${ }^{147}$ Perhaps we should further demand that $a$ is interpreted as a name of an object that is extended to make sure that it makes sense to predicate greenness or non-greenness of it. The Eiffel Tower certainly is such.

148 This point extends in the obvious way to logical consequence.

149 This seems to be the key assumption behind Cocchiarella's criticism of Kripke Semantics: Kripke Semantics incorporates material conditions into the semantics of $\square$ and can consequently not capture a reading of the latter under which it expresses a purely formal concept Cocchiarella [1975: 13ff]. However, I should like to remind the reader that we are not necessarily seeing ourselves as responding to Cocchiarella's criticism. Instead, we are merely pointing out why, from our present modeling perspective, we can not demand that the truth of $\square \alpha$, in all models, is decided relative to all possible first-order structures.
} 
of the possible way the world could have been which that model represents.

Consider now the example again: if anything is true in virtue of form in the requisite sense, it should be the statement 'The Eiffel Tower is either green or not green'. After all, it appears to just be an instance of the law of excluded middle. The proponent of the (AFP)-approach would have to exclude 'is green or not green' as a legitimate interpretation of the predicate $F$. From the perspective of logical atomism it should be immediately clear where the problem lies: 'is green or not green' is not a logically atomic expression. This way of making sense of the (AFP)-approach, therefore, relies on a certain relationship between the formal and natural language: A valid sentence, in the model theory, is one such that it is true in all possibilities under all interpretations of the non-logical terms, where the interpretations of the various parts of the formal language should be expressible using natural language terms that are logically atomic. There may be natural language interpretations of $\diamond \neg F a$ where it expresses a falsehood, but not so if the interpretations can be given using only logically atomic natural language expressions. 'Is green or not green' is clearly not a logically atomic natural language expression, by any standards, and our interpretation of $\diamond \neg F a$ as 'It is logically possible that The Eiffel Tower is both green and not green' is illegitimate. On the other hand, if $\mathcal{N}$ is a natural language predicate that is truly logically atomic, statements of the form 'It is logically possible that $a$ is not $\mathcal{N}$ ' should all be true.

There are essentially two ways of making sense of the notion of being logically atomic which is currently employed to demarcate the relationship between the formal language validities and the natural language statements which they are meant to mirror.

(A) The notion of being logically atomic is decided relative to the notion of logical form exhibited by the formal language for which we are currently developing a model-theoretic account of consequence and validity. 
(B) The notion of being logically atomic is decided relative to an absolute notion of logical form.

Suppose we accept (A). The notion of logical form by which we decide whether a given natural language interpretation is legitimate is given by the notion of logical form exhibited by the formal language in question. The first thing we may note is that this seems hopelessly circular. To know whether a certain natural language expression is logically atomic we must already know its logical properties when it is formalized and its behavior within the model theory. But this presupposes a clarification of the notion of formalization which is just a codification of which natural language expressions can correspond to which formal language terms; and this latter question was what was at stake in the first place. I will not dwell on such issues here. ${ }^{150}$ Instead I will point out that option (A) does not let us uphold that a valid formula is one that is true in all possibilities under all interpretations, and so, to ensure this, we ultimately end up with the notion of logical atomicity given in (B).

To clearly articulate this point, consider for the moment a propositional language augmented with an operator $\square$ to mean logical necessity. If we develop a model theory for this language in accordance with $(\mathrm{AFP}),{ }^{151}$ it should be clear that $\diamond \neg p$ will be a validity. However, there are natural language statements that would count as a legitimate interpretation of $p$, in accordance with $(\mathrm{A})$, yet under which the result would not be true. These are statements that do not exhibit any logical form modulo what can be expressed using the natural language counterparts of the logical constants of a propositional language, such as 'All bachelors are bachelors'. The result of interpretating $p$ in this manner in $\diamond \neg p$, 'It is logically possible that some bachelor is not a bachelor', is presumably false. But $\diamond \neg p$ is true in all models, and so we do not

\footnotetext{
${ }^{150}$ Let it be noted, though, that I do not wish to claim that this circulatiry is necessarily vicious.

${ }^{151}$ By which we mean that for each possible valuation of the atomic sentences, there will be an index $w$ in each model such that $I(w)$ is that very valuation.
} 
have an equivalence of being true in all models and being true in all possibilities under all interpretations given in accordance with (A). The problem, of course, is that 'All bachelors are bachelors' is a logical truth yet the formal language does not have the resources to express this in terms of its logical constants. 'All bachelors are bachelors' will show up as a logical truth in the formal language only when we move to a quantified language (i.e., formalized as $\forall x(B x \rightarrow B x)$ ), but under our current conception of what it is to be logically atomic, and so what counts as a legitimate interpretation, 'All bachelors are bachelors' does count as such since its logical form cannot be given in a propositional language. This, obviously, means that our model theory is not capturing the notion of being true in all possibilities under all logically atomic interpretations of the non-logical terminology, where the content of what it is to be logically atomic is given by (A) above.

At this point it will obviously be pointed out that we ought to move to a quantified modal language when considering interpretations like those of 'All bachelors are bachelors'. This much should be granted in a relatively unproblematic fashion, though it should be kept in mind that this does not solve the issue for propositional languages as the above conclusion still stands.

And even if we do grant that we should move to a quantified language the same problem recurs, though since our formal language has now gained more expressive power when it comes to its logical constants, there will, all else being equal, be fewer clear-cut counterexamples. Consider, say, the property of having all the properties which Napoleon has. This, it seems, is a perfectly legitimate first-order property, one which every individual either has or does not have. But, once again, it seems we have interpretations of $F$ and $a$ (i.e., as being the property of having all the properties which Napoleon has and as a name for Napoleon) such that $\diamond \neg F a$ is false, as the natural language statement 'It is logically possible that Napoleon does not posses all 
of the properties which he has' clearly seems to be false. There are various ways, I suppose, of trying to counter this intuition. ${ }^{152}$ One is to just deny that $\diamond \neg F a$ is false under this interpretation. I am unsure that this can be maintained. After all, it seems that a proper formalization of 'Napoleon has all the properties which Napoleon has' in a second-order language would be $\forall X(X n \rightarrow X n)$, which is certainly true in all models, so 'Napoleon has all the properties which Napoleon has' seems to be logical truth. But even if it is not the case, we can see how this way of responding ultimately relies on there not being any logical truths which can only be expressed once we have available the resources of a second-order language. What this really amounts to, then, is a reliance of an absolute notion of logical form, which is given by the natural language counterparts of the logical constants of a first-order language. We want our model-theoretic definition of consequence to capture truth preservation in all possibilities under all logically atomic interpretations of the non-logical terminology. To make sure that there are no counterexamples to this we had to dismiss propositional languages as not having the recourses to express the true logical form of given natural language expressions. And now, to make sure that there are no counterexamples in a first-order language, we have to rely on the claim that a first-order language is capable of expressing everything that can properly be said to be part of the logical form of an

\footnotetext{
152 One way of doing this, which I will not discuss in any detail, is to claim that 'the property of having all the properties which Napoleon has' should really be regarded as a rigidified description picking up its reference at the world in which it is used. That is, the property of having all of the properties which Napoleon has is to be understood as having all of the properties that Napoleon actually has. Since it is surely possible for Napoleon to have lacked one of the properties which he actually possesses, the interpretation of $\diamond \neg F a$ as 'It is logically possible that Napoleon does not posses all of the properties which he has' is not actually false, since it is to mean that it is logically possible that Napoleon could have lacked a property which he actually has. Pursuing this line of reasoning would engage us in a debate as to whether the modal profile which a logically valid argument, or in this case, logically valid sentence, should have is of a metaphysical or epistemic nature. We can know a priori that whenever the statement 'Napoleon has all the properties which he actually has' is made, it will be true. This follows from the assumption that actually, as used here, refers to the circumstances of utterance or inscription. Yet, there will be metaphysically possible scenarios - what is sometimes referred to as counterfactual scenarios - where what is expressed by 'Napoleon has all the properties which he actually has' is false. This happens when the circumstances of evaluation are different from those of the utterance or inscription of the statement.
} 
expression. ${ }^{153,154}$

In order to shield myself from being misunderstood let me state that I am not claiming that such a claim could never be defended. Quine famously argued that second-order logic is really "set-theory in sheep's clothing" and thus not logic proper (Quine [1970: 66]). From such a perspective, we could welcome the conclusion that any proper logical truth can be captured using the reasources of a first-order language.

The point which I am making here is that if we want to see our model theory as capturing a notion of truth preservation in all possibilities under all interpretations of the non-logical terminology, then we should not decide the truth of $\square$-claims, in

\begin{abstract}
${ }^{153}$ We may note that it is in fact not quite sufficient for the proponent of the (AFP)-approach to hold that a valid argument is one such that it is truth preserving under all logically atomic simpliciter interpretations. If this is the only restriction, a formula such as $\diamond \neg(F a \rightarrow G a)$ would be a counterexample to the supposed equivalence of being true in all models and being true under all such interpretations. Consider the interpretation of $F$ and $G$ as the same logically atomic simpliciter natural language expression, say, 'is red', and $a$ as a name for, say, the Eiffel Tower: under this interpretation the formula, which is true in all models, expresses what 'It is logically possible that it is false that if the Eiffel Tower is red, then the Eiffel Tower is red' expresses. And the former clearly seem false. If anything is logically necessary, statements of the form 'If $A$, then $A$ ' surely seem to be such, for arbitrary natural language statements $A$. So we must further demand that different terms of the formal language receive different logically atomic simpliciter interpretations. We are thus relying on a one-to-one correspondence between natural language and the formal language: each atomic formula of the formal language correspond to one, and only one, logically atomic simpliciter natural language expression.
\end{abstract}

${ }^{154}$ At this point the proponent of (AFP) may point out that we are misrepresenting the situation. We have focused on the claim that there are certain interpretations of the non-logical terminology that are not represented by the model theory, and that consequently, we are not modeling a phenomenon of being true (or truth preserving) in all possibilities under all interpretations of the non-logical terminology. Although our perspective here is another, it might be claimed that the models are not meant to give (or represent) interpretations of the non-logical terminology, and that because of this, there are no interpretations missing. Instead, we are meant to think of the non-logical terms of our formal language as already enjoying an interpretation and the different models as representing possible ways the world could have been relative to these interpretations. It should be relatively clear though, that we are not solving the issues, since the interpretations which the non-logical terminology now enjoy, though stable across models, are still compatible only with interpretations where they correspond to logically atomic natural language expressions. For the reasons we adduced above, all non-logical terms will be represented by any model to have an interpretation under which it is logically atomic: this fact does not change depending on whether we see the models as providing that interpretation, or as simply respecting such an interpretation. The non-logical terminology of the formal language represent, on this approach, only logically atomic natural language expressions. The problem, as it were, is thereby moved to the translation process where which natural language arguments are given which formal renderings will depend on a demarcation of the logically atomic natural language expressions from the logically complex. 
all models, relative to all possible first-order structures for the non-modal part of the language, as (AFP) would have it. If we do so from our present perspective, we are capturing a notion of being truth preserving in all possibilities under all logically atomic simpliciter interpretations of the non-logical terminology.

At this point, then, we may naturally ask whether there is any gain in capturing the latter notion as opposed to the former. As I shall attempt to point out, the opposite seems to be the case. Even if we have a perfectly good theory of what it is to be a logically atomic simpliciter natural language expression, we may not want to develop our model-theoretic definition of consequence in accordance with this, in order for our theory of consequence to apply as generally as possible.

Can it be maintained that we ought to have a division between being logically atomic and logically complex in mind when developing a formal theory of consequence? Here we can call on support from Quine. In correspondence with Carnap (Creath [1990]), Quine responds to a question from Carnap to the effect of whether Quine would go along as to claim that certain formula should be atomic. ${ }^{155}$

$[Y]$ ou are right in presuming I do not agree. My reasons for not taking the difference between atomic and non-atomic matrices as relevant for the logical form:

(a) (the less important reason): It seems natural to say that all statements got by substitution for letters in a schema share the logical form depicted by the schema (along with the logical forms of any more complex schemata from which the several statements are derivable by substitution).

(b) (the more important reason): To make logical form depend on [an]

\footnotetext{
155 Carnap suspects as much; he writes "I myself should take a further step (as you presume) in which you probably will not follow me. I should add the specification that the antecedent and therefore the consequent are atomic matrices" (Carnap to Quine, August 1943; in Creath [1990]) The exact nature of the problem is not terribly important for our present purposes; Quine's denial of the distinction between logically atomic and not logically atomic as it determines logical form is.
} 
idea of atomicity is to presuppose completion and adoption of one specific constitution system for the empirical language in which the logic is applied; for it is only in terms of such a constitution system that we can distinguish between the truly atomic and the apparently (via definitional abbreviations) atomic. (Quine to Carnap, September 1943, in Creath [1990])

What matters most for our present purpose is Quine's second, and in his own words, more important reason. What Quine is pointing out in the above quote is the problem of presupposing a logical form simpliciter, and that in a case where we can work with logical form and hence logical atomic expressions, we would have to know which apparently atomic expressions are really abbreviations of more complex ones. ${ }^{156}$ To know whether a natural language statement is logically atomic simpliciter presupposes, as Quine points out, an adoption of a single constitution system for the language. We need a categorization of all terms of the language in accordance with which each term belongs to a specific category, and, perhaps more pressingly, we would need a designation of the natural language terms that are the true logical constants, the natural language terms whose formal counterparts are not to vary in the interpretation given to them. Consider the argument:

- Wisdom is admirable.

- If wisdom is admirable, redness is a color.

- Therefore, redness is a color.

Now, it seems to me that this argument is clearly valid. (Again, it is just an instance of modus ponens!). However, 'wisdom' and 'redness' as they are being used here are not referring to simple individuals but properties, and in turn, 'is admirable' and 'is a

\footnotetext{
${ }^{156}$ See the below example of 'is a bachelor' and 'is unmarried' for a spelling out of this latter concern.
} 
color' are being used as second-order properties. The proper logical form simpliciter of these statements are thus such that they are not logically atomic and therefore do not count as interpretations of any first-order sentences. The upshot is that if our model theory captures a notion of being truth preserving in all possibilities under all logically atomic simpliciter interpretations, the validity of this argument will not be explained in a simple first-order language by pointing out that the argument from $F(a)$ and $F(a) \rightarrow H(b)$ to $H(b)$ is valid. Instead we must turn to a second-order language to explain it. This puts unnatural limitations on the explanatory power of our theory. Consider a valid formula of a quantified modal language such as $\square(F(a) \vee \neg F(a))$. We can now ask, as we saw Burgess [2003: 147] do in the propositional case, what we mean to endorse by saying that this formula is a validity? Do we mean to endorse that it could not fail to be the case that any property expressed by a logically atomic predicate either is had or not had with logical necessity by any given object, or do we mean to endorse that it could not fail to be the case that any property, however it can be expressed, either is had or not had with logical necessity by any given object? I take it that we take a formula such as the above to intuitively be expressing the latter. When doing logic, we aim to be as general as possible when circumscribing the class of logical truths. Of course, there is no inconsistency in maintaining that what we mean by endorsing this simply is that it could not fail to be the case that any property which can be expressed by a logically atomic predicate is either possessed or not by any given object with logical necessity. But if we want its validity to represent that it could not fail to be the case that any property is either had or not had with logical necessity by any object, then we should see our model theory as capturing a phenomenon of being true in all possibilities under all interpretations of the non-logical terminology, not merely all logically atomic simpliciter interpretations. 
Another thing which we could naturally wonder, is whether there are any two natural language predicates, such that the application of one logically necessitates the application of the other, but where this logical dependence cannot be expressed in the language. It might be argued that 'is a bachelor' and 'is unmarried' are two such predicates: that it is logically necessary for anything that is a bachelor to be unmarried. $^{157}$ If this is the case, then we see how a valid formula such as $\diamond \neg \forall x(F x \rightarrow$ $H x)$ has false a false interpretation. In order to deny this we then have to claim that one of these is not properly logically atomic and is instead a definitional abbreviation of the other. But if this is the case then we cannot explain the validity of the following argument

$\left(\mathrm{P}_{1}^{\prime}\right)$ It is logically possible that James is a bachelor.

$\left(\mathrm{P}_{2}{ }^{\prime}\right)$ It is logically necessary that whoever is a bachelor is unmarried.

(C') It is logically possible that James is unmarried.

by pointing to the formal validity of the argument

$\left(\mathrm{f}-\mathrm{P}_{1}^{\prime}\right) \diamond F(a)$

$\left(\mathrm{f}-\mathrm{P}_{2}{ }^{\prime}\right) \square \forall x(F(x) \rightarrow H(x))$.

$\left(\mathrm{f}-\mathrm{C}^{\prime}\right) \diamond H(a)$

'is a bachelor' and 'is unmarried' are not proper natural language interpretations of the non-logical constants in this argument, since 'is a bachelor', under our current conception is to be expressed as a complex predicate, a component of which is 'is unmarried'. Presumably we could define ' $\mathrm{x}$ is a bachelor' as ' $\mathrm{x}$ is unmarried and $\mathrm{x}$

\footnotetext{
${ }^{157}$ At least under the assumption that the notion of logical necessity which we are interested in is that of being true in all possible ways the world could have been in virtue of the semantics of the terms.
} 
has never been married and $\mathrm{x}$ is a man'. And rather than point to the above formal argument as an explanation of the validity of the natural language argument, we would have to call upon

$\left(\mathrm{cf}^{\left.-\mathrm{P}_{1}{ }^{\prime}\right)} \diamond(U(a) \wedge N(a) \wedge M(a))\right.$

$\left(\mathrm{cf}-\mathrm{P}_{1}{ }^{\prime}\right) \square \forall x((U(x) \wedge N(x) \wedge M(x)) \rightarrow U(x))$.

$($ cf-C') $\diamond U(a)$.

instead. ${ }^{158}$ If our model theory can explain the validity of

$\left(\mathrm{P}_{1} "\right)$ It is logically possible that James is happy.

$\left(\mathrm{P}_{2}^{\prime \prime}\right)$ It is logically necessary that whoever is happy is singing.

(C") It is logically possible that James is singing.

by pointing to the formal validity of the argument from ( $\left.\mathrm{f}^{-} \mathrm{P}_{1}{ }^{\prime}\right)$ and $\left(\mathrm{f}-\mathrm{P}_{2}{ }^{\prime}\right)$ to (f-C'), then should we not also be able to explain the validity of the first argument by pointing to this? By denying this we are forcing a non-uniform treatment of the relation between natural language arguments and the validity of formal arguments, because, as Quine also points out, in order to know which formal arguments explain the validity of which natural language arguments, we will have to know the true logical form of the involved expressions, in this case 'is a bachelor' and 'is unmarried'. So even if logical atomism provides us with a true theory of the world, we might still not want to hold our theory of logical consequence hostage to the resulting notion of logical form. We presumably want to be able to explain the validity of all arguments in modus ponens form by pointing to the valid propositional argument $\langle\{p \rightarrow q, p\}, q\rangle$. Intuitively, what this tells us is that for any natural language interpretations of $p$ and $q$ we are dealing with

\footnotetext{
158 Note, incidentally, that the second premise is now unnecessary, since it reflects the true logical form of 'is a bachelor'. And since this form includes 'is unmarried' it becomes itself a validity.
} 
a valid argument. And we can be sure of this since, whatever the true logical form of $p$ and $q$ are, given the meaning of 'if... then...' the premises cannot be true while the conclusion is false. If we accept the relationship between the formal and natural language that relies on a notion of absolute logical form, we lose this explanation, except for the case of truly logically atomic interpretations.

\subsection{The Intended Frame}

In the case of propositional modal languages, we suggested that there are two ways to make sense of the claim that all logically possible worlds should be in $W$. In the sense intended by Cocchiarella and Hintikka, it means that all possible valuations of the atomic sentences are in the range of the quantifier in the truth clause for $\square \alpha$ in order for it to be true. We argued that this demand is mistaken from the point of view of capturing a phenomenon of truth preservation in all possibilities under all interpretations, as it places restrictions on the interpretations which we survey. In the above section we looked at the corresponding claim for quantified languages, a claim that as a very minimum all first-order models of a given size should be among the alternatives to a given world, and we argued that forcing these restrictions has the same kind of consequences as in the propositional case.

We also briefly looked at another way of making sense of the claim that $W$ should contain more indices. On this view, the problem is that $W$ should reflect something about the structure of the modal space which we are interested in. In the case of propositional languages we pointed out that insisting that $W$ be a certain size will not change anything about the valid arguments and sentences of the semantics, and we suggested that there is little sense in seeing a model where $W$ is a certain size as representing something which cannot be represented by a model where the set of 
indices $W^{\prime}$ is such that $W^{\prime} \subset W$, but the two models nevertheless make exactly the same sentences true. But one might think that the situation changes when moving to quantified languages, since each index now has representational capacities that extend that of simply assigning a truth value, namely, the associated domain $\psi(w)$.

Hanson \& Hawthorne [1985] point to a puzzle in standard Kripke Semantics for modal logics.

The puzzle is why more than one model structure is used in the definition of validity. There is presumably just one class of all possible worlds and one model structure defined on this class that does correctly the things that model structures are supposed to do. [...] Why not define validity simply as truth at every world under every model on this one model structure? What is the point of bringing in more model structures than just this one? $(\mathrm{H} \& \mathrm{H}[1985: 9])$

This worry is not quite the same as that of Cocchiarella and Hintikka, though one could be forced to adopt one frame (what H\&H call 'model structure') if one were to accept that the truth clause for $\square$ should always range over all possible first-order structures. The puzzle, as H\&H see it, is not restricted to readings of $\square$ under which it is to mean logical necessity. Rather, the intuition seems to be that if the modal space of interest has a certain structure, then there seems to be no reason not to use the frame which represents this structure. H\&H define a model structure, which we will call 'a frame', as a tuple $\left\langle W, @, R_{1}, \ldots, R_{n}, \psi, D\right\rangle$, where each $R_{i}$ is a relation on $W .{ }^{159}$ The intended frame, $\mathrm{H} \& \mathrm{H}$ suggest, lets us view interpretations as being genuine meaning assignments.

\footnotetext{
159 Though, following Kripke [1963], they denote the index set $W$ by ' $K$ ' and the designated index @ by ' $G$ '. I have made the requisite substitutions when quoting H\&H [1985].
} 
$[$ W]e should restrict our attention to just one model structure, call it $\mathcal{S}$, in which $W$ is the class of all possible worlds, @ is the actual world, $\psi(w)$ is the set of all things that exist in $w$, for each $w \in W$, and each $R_{i}$ is a relation between possible worlds (a world accessibility relation) that forms the basis of a recursion rule for one of the non-truth-functional connectives[.] [1985: $11]$

About the intended frame, $\mathrm{H} \& \mathrm{H}$ says:

[T] he intended model structure $\mathcal{S}$ is eminent among model structures for intensional languages, for it is central to the theory of meaning for such languages. This centrality is reflected in three ways. First $\mathcal{S}$ provides the basis for valuation functions that are genuine meaning assignments (not just functions defined on some arbitrarily selected index set) and which therefore determine an extension for each nonlogical symbol at each world. Second, since the actual world is an index of $\mathcal{S}$, each meaning assignment defined on $\mathcal{S}$ determines what we might call the extension simpliciterthe extension at the actual world - of each nonlogical symbol. Finally, the accessibility relation of $\mathcal{S}$ fix the meanings of the intensional connectives and prevent them from varying from model to model. [1985: 12]

$\mathrm{H} \& \mathrm{H}$ are pointing out that when we work with the intended frame, we can see the valuations as genuine meaning assignments. At the same time, they urge, fixing the relations $R_{i}$ one prevents the meaning of the corresponding operator to vary from model to model. This is to be seen in relation to a Kripke Semantics, where the set $W$ and the relations $R_{i}$ are allowed to vary within some minimal conditions which they must meet: non-emptiness for $W$, and typically reflexivity, transitivity, and the like for $R_{i}$. This, it may be thought, allows variation in the meaning of $\square$ between Kripke Models. 
But this should not occur, since the meaning of $\square$ is supposed to be fixed.

As for the former point, about genuine meaning assignments, $\mathrm{H} \& \mathrm{H}$ are certainly right that a valuation on a frame which represents the actual modal space of interest are much more interesting in that they can be seen to correspond with actual meanings. The idea here is once again to equate the meaning of a term with its intension: a function from worlds to extensions. However, as noted by $\mathrm{H} \& \mathrm{H}$, it is unclear what the class of all possible worlds is supposed to be. Here, H\&H allow for some idealization.

There is, however, a potentially unsatisfactory feature of these definitions[.] This is the fact that they all refer to the model structure $\mathcal{S}$ whose index set $W$ is the set of all possible worlds. Since the nature and number of possible worlds are matters of dispute and puzzlement, formal work in the semantics of intensional languages may seem all but impossible if $\mathcal{S}$ and $W$ are understood in this way. We think that the proper response to this worry is the one we mentioned earlier in connection with extensional languages: some idealization of the notion of a possible world is needed if semantics is to yield any interesting results, and that notion should be construed broadly rather than narrowly. Using this general technique we should seek to determine the formal constraints $\mathcal{S}$ and $W$ must satisfy in order for interesting theorems to hold, and then ask ourselves if these constraints count for or against a particular metaphysical view of possible worlds. [1985: 13]

$\mathrm{H} \& \mathrm{H}$ are here suggesting that a certain idealization of the notion of a possible world is needed if we want the resulting semantics to give us interesting results. This is certainly an interesting claim in that one can hardly deny that Kripke Semantics, whose notion of validity $\mathrm{H} \& \mathrm{H}$ find lacking in motivation, has yielded many interesting results. In the last line of the quote they seem to almost suggest that interesting results in the 
formal semantics can inform our decision as to what metaphysical notion of a possible world we are to adopt. We will come back to the notion of idealization involved.

$\mathrm{H} \& \mathrm{H}$ propose the following constraints on $\mathcal{S}$ and $W$. "W is at least denumerable, and that for each nonzero countable cardinal $\tau$, there are at least denumerably many $w \in W$ such that the cardinality of $\psi(w)$ is $\tau "$ [1985: 14]. Since H\&H do not return to the matter, in the light of the above quote we can only assume that they find their technical results under these assumptions interesting enough to justify this understanding of $\mathcal{S}$. Following $\mathrm{H} \& \mathrm{H}$, the intended frame, assuming a universal accessibility relation for our $\square$-operator understood as logical necessity, becomes:

$\mathcal{S}=\langle W, @, \psi, D\rangle$, where the class $W$ is at least denumerable and for each nonzero countable cardinal $\tau$, there are at least denumerably many $w \in W$ such that the cardinality of $\psi(w)$ is $\tau$.

We will suggest that for the semantics of $\square$, understood as logical necessity, these genuine meaning assignments which can be given on $\mathcal{S}$ do not play any definitive role in assigning truth to $\square \alpha$. The criticism here is not that $\mathcal{S}$ cannot form a basis for genuine meaning assignments, though somewhat idealized. From the perspective of modeling a notion of consequence some idealization is arguably needed in all cases, and the assumptions which $\mathrm{H} \& \mathrm{H}$ make seem plausible in order to be able to have a enough representatives of worlds in order to model the notion of interest. If every possible world (of the requisite kind) is represented in $W$, we can simply equate the intension of a term (that is, a function from possible worlds to extensions) with its meaning, though for obvious reasons, whatever idealization is involved in circumscribing $\mathcal{S}$ will be inherited by these meaning assignments.

By surveying all possible intensions of the non-logical terminology on the frame $\mathcal{S}$ we are surveying all genuine meaning assignments to the non-logical terminology. But for the language augmented with a $\square$-operator to mean logical necessity, these genuine 
meaning assignments do not play a deciding role. Let us therefore turn to H\&H's definition of validity for such a language. ${ }^{160}$

The semantics is again defined on the intended model structure $\langle W, @, \psi, D\rangle$. The meaning assignments are the valuations $v$ such that: for each proposition letter $\alpha$ and $w \in K, v(\alpha, w) \in\{t, f\}$; for each n-ary predicate letter $\alpha$ and and $w \in W, v(\alpha, w) \subseteq \psi(w)^{n}$. Note that for $Q L$ a meaning assignment to a predicate letter $\alpha$ always specifies the extension of $\alpha$ at a world as a collection of things in that world. Assignments of things to free variables are functions $s$ such that for every $x$ and $w \in W, s(x, w) \in \psi(w)$. These functions are like meaning assignments in that what they assign to a symbol at a world is always something from that world. [1985: 20]

The language $Q L$ does not contain individual constants, but there is no principled reason why we could not extend it to include them. $\mathrm{H} \& \mathrm{H}$ define the extension of a given predicate at a given world to be objects or sets of objects existing in that world, and similarly for variable assignments. Again, there is no principled reason why we could not let go of this assumption.

After giving the standard definition of satisfaction of a formula $\alpha$ at $w$ under a valuation $v$ and variable assignment $s$ - which they write as $\operatorname{sat}(\alpha, v, s, w)$ - for a formula whose main connective is not modal, they give the, for our purposes, interesting clause for $\square \alpha$.

(ln) $\operatorname{sat}(\square \alpha, v, s, w)$ iff $\forall v^{\prime} \forall s^{\prime} \forall w^{\prime} \operatorname{sat}\left(\alpha, v^{\prime}, s^{\prime}, w^{\prime}\right)$.

The interesting fact here is that the truth of $\square \alpha$ is not defined relative to the valuation currently given on $\mathcal{S}$. Instead, reference is made to every world in every model definable

\footnotetext{
${ }^{160} \mathrm{H} \& \mathrm{H}$ denote their operator $L$, and say that $L \alpha$ stands for the logical necessitation of $\alpha$ ([1985: $19])$.
} 
on $\mathcal{S}$, which, in accordance with the assumptions made about $W$ and $\psi$ in $\mathcal{S}$ is just to say that $\square \alpha$ is true at a world under a given valuation if and only if $\alpha$ is true on every domain under every valuation, where occurrences of $\square$ in $\alpha$ take on the same recursive meaning. The genuine meaning assignments qua intensions do not play a role in deciding the truth of $\square \alpha{ }^{161}$ From the perspective of stressing the importance of the (idealized) genuine meaning assignments, one would have expected that they were to play a role. In fact, one would expect genuine meaning assignments viewed as extensions in all possible worlds to play a role because they can decide the truth of a claim such as $\square \alpha$, whereas a mere extension cannot.

H\&H's proposed semantics essentially incorporates the assumption that the quantifier in the truth clause for $\square$ claims ranges over all first-order models. This is even stronger than the claim that each model with the same domain is in the quantifier range, though it of course incorporates this latter claim. For this reason the proposed semantics is subject to the same limitations as pointed out above. If our aim is to capture a notion of truth preservation in all possibilities under all interpretations, then there will be some interpretations that are not represented.

To really make use of the (idealized) genuine meaning assignments in defining the truth of $\square \alpha$, we should presumably define the truth of each claim of the form $\square \alpha$ relative to just one assignment of intensions on $\mathcal{S}$ for the non-logical part of the language. It is such an intension qua (idealized) genuine meaning assignment which tells us whether $\alpha$ means something (in a given model) that is true in all logically possible worlds, and consequently whether $\alpha$ is logically necessary, that is, whether $\square \alpha$ is true. But if we define the truth of $\square \alpha$ relative to just one valuation on $\mathcal{S}$, we are effectively just defining truth in a model for the Kripke Model based on the frame $\mathcal{S}$.

$\mathrm{H} \& \mathrm{H}$ go on to define a notion of truth at a world under a valuation, analytic truth

161 This is not strictly speaking true, since all possible valuations play a role in deciding the truth of $\square \alpha$. What we mean is that it does not play a prominent or decisive role. 
under a valuation, and logical truth.

- $T(\alpha, v, w)$ iff $\forall s \operatorname{sat}(\alpha, v, s, w)$.

- $A T(\alpha, v)$ iff $\forall w T(\alpha, v, w)$.

- $L T(\alpha)$ iff $\forall v A T(\alpha, v)$.

The validity of a formula is defined to coincide with logical truth; i.e., $v_{a l}(\alpha)$ iff $L T(\alpha) . \quad \mathrm{H} \& \mathrm{H}$ refers to their notion of validity as 'validity' ${ }_{1}$ and argues that it is better motivated than validity ${ }_{2}$ which is identified as truth at every index under every valuation on every model structure, but where the truth of $\square \alpha$ is decided relative to only one valuation ([1985: 14]). H\&H treat standard Kripke Semantics as a prime example of a semantics that delivers the notion of a valid 2 sentence. We may note, in relation to this, that the most prominent difference between the two approaches, when logical necessity is at stake, is not that validity ${ }_{2}$ makes use of more than one frame. The difference which sets the two apart is rather that the clause given in (ln) makes reference to all valuations, and is thus not restricted to the ones given in the model in question. In fact, applying the clause (ln) as well as the definition of truth, analytic truth, and logical truth, to all models built on all frames, would yield minimal changes to the output of valid sentences. ${ }^{162}$ What really sets the Kripke Semantics apart from the alternatives is that the truth of $\square \alpha$ is decided relative only to valuations that are present in that very Kripke Model. If the collection of models are meant to represent all different interpretations of the non-logical terminology (from all possible ways the world could have been), this is as it should be. We want interpretations of the language where some formulae are logically necessary or depend for their truth upon the truth

\footnotetext{
${ }^{162}$ Sentences like $\diamond \exists x, y(x \neq y)$, which are valid 1 would no longer be valid because of the frame which has $|\psi(w)|=1$, for all $w \in W$. But, as far as I can tell, these kinds of cardinality sentences would be the only ones affected.
} 
of other formulae. These interpretations come about when $\square \alpha$ is decided relative to just one valuation on one frame.

Though $\mathrm{H} \& \mathrm{H}$ never explicitly define exactly what they mean by validity 2 they do state that standard Kripke Semantics gives rise to this notion ([1985: n.4]). Let us therefore decide to denote by the use of 'validity 2 ' validity in a standard Kripke Semantics - often referred to as universal validity - while keeping in mind that what sets the two notions apart is not only the use of more than one frame. Essentially, what sets them apart, is the fact that in Kripke Semantics the truth of $\square \alpha$ is decided relative to a single model. The truth clause for $\square$ does not, as in the alternative, require us to consider all valuations that can be defined on $\psi(w)$, for $w \in W$.

\subsubsection{Validity Vs. Validity $_{2}$}

If $K$ and $\psi$ of the single model structure $\mathcal{S}$ involved in validity ${ }_{1}$ can be specified in a way that makes $W$ plausibly represent the set of all possible worlds, then validity 1 is far more intuitively satisfying that validity . We think our comments on truth, analyticity, logical truth, and validity ${ }_{1}$ in the previous section make this clear. (H\&H [1985: 14]).

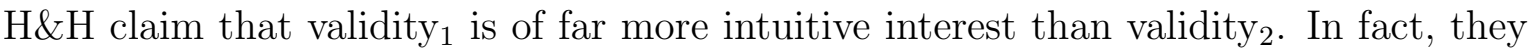
go on to claim that it is hard to discover any rationale behind validity 2 , something we will look at shortly.

To motivate validity, $\mathrm{H} \& \mathrm{H}$ introduce the notion of an $I$-interpretation:

[W]e define an interpretation of $L$ (also called an $I$-interpretation) as an ordered pair $(v, w)$ consisting of a valuation defined on $\mathcal{S}$ and a world $w$ from the index class $W$ of $\mathcal{S}$. An $I$-interpretation is similar to an $E$ interpretation in that it provides a set that functions as a domain of quantification and an assignment to each nonlogical symbol of the language. For 
the $I$-interpretation consisting of the valuation $v$ and the world $w$ the set in question is $\psi(w)$, and the value assigned to a nonlogical symbol is the intension of that symbol under $v$. [1985: 12]

An $E$-interpretation, as $\mathrm{H} \& \mathrm{H}$ use the term here, is just an extensional first-order model; a domain along with an assignment of extensions to the non-logical constants. An $I$ interpretation is supposed to be the extension of this notion to intensional languages, in that it assigns an extension to a given term at all worlds.

However, for the semantics where $\square$ is to mean logical necessity, it does not play the central role which $\mathrm{H} \& \mathrm{H}$ make it out to. It is completely irrelevant to the truth of any given formula, $\alpha$, what is assigned to the non-logical symbols in other worlds under that very $I$-interpretation. If $\alpha$ is non-modal, the truth value of $\alpha$ at $w$ will be determined completely on the basis of the extensions of the non-logical terms, and though this is in fact given by $v$ at $w$, any $v^{\prime}$ agreeing with $v$ about the denotations at $w$ would produce the same result. The extending feature of an $I$-interpretation, i.e., that it assigns denotations in all worlds, is therefore irrelevant, and an E-interpretation given at $w$ would do just as well. Of course, since $\alpha$ is non-modal this is to be expected. But consider now $\square \alpha$, understood as it is logically necessary that $\alpha$. The truth of this at a given index will still not be determined by what $v$ assigns to the non-logical parts of $\alpha$ in other worlds. All assignments to $\alpha$ 's non-logical parts which can be given in any first-order structure decide the truth of $\square \alpha$, and the given $I$-interpretation does not play an essential role in deciding the truth of $\square \alpha{ }^{163}$

At this point it is likely that $\mathrm{H} \& \mathrm{H}$ will reply that this is so because logical necessity is supposed to be a purely formal operator, a claim which we have seen motivate Coc-

\footnotetext{
163 This claim should be qualified slightly: it is not that what $v$ assigns to the non-logical part of $\alpha$ at other worlds in $w$ does not matter at all, since $v$ 's assignments in these other worlds will represent some first-order models. The point is that it is actually completely irrelevant what the specific $v$ assigns to the non-logical terminology in other worlds: even if a certain first-order model is not represented, the truth of $\alpha$ will still depend on the latter.
} 
chiarella into criticizing the treatment of $\square$ as logical necessity in Kripke Semantics. It is clear that from our present perspective H\&H's alternative presupposes the same notion of being logically atomic simpliciter when it comes to explaining the relationship between formal and natural language. The reasons are the same as those given in previous sections: if the truth clause for $\square$ is forced to range over all first-order structures, then we are excluding some interpretations of the language that can only be deemed illegitimate if we assume a relationship between natural language and formal language that relies on a notion of being logically atomic simpliciter. In the previous sections we gave some reasons why we might not want to rely on such a notion of logical form, and at any rate, we pointed out that it does not provide a standpoint from which we can claim that there is something wrong, in any absolute sense, with a Kripke Semantics.

Assuming that we are out to capture necessary truth preservation under all interpretations, we are in disagreement with $\mathrm{H} \& \mathrm{H}$ about the correct semantics for $\square$ when it is understood as logical necessity. We may note, as well, that once we accept that the truth of $\square \alpha$ should be decided relative to just one valuation on $\mathcal{S}$, it is unclear that we are dealing with a semantics that is, so to speak, in direct opposition to a standard Kripke Semantics. The treatment of claims of the form $\square \alpha$ just is the standard treatment which they receive in a Kripke Semantics, though we are now putting more restrictions on the models than the usual one that $W$ be non-empty. From this perspective, of course, Hanson \& Hawthorne's criticism does not show that there is anything fundamentally mistaken about the approach taken in standard Kripke Semantics, but can at most show that there are further restrictions which we ought to put on the notion of a model. These further restrictions are the ones reflected in the conditions put on the intended frame, $\mathcal{S}$.

$\mathrm{H} \& \mathrm{H}$ claim that it is hard to discover any rationale for validity ${ }_{2}$, though when it comes to their system for nomic necessity, they weaken this claim: 
The treatment of "contingent necessity" in the system for nomic necessity suggests a reason for continued interest in validity ${ }_{2}$ [1985: 19]

This suggests that the reason for accepting H\&H's notion of validity ${ }_{1}$ lies in its capability to capture the correct semantics when logical necessity is at stake.

Our disappointment with studies involving validity 2 is, primarily, that little thoughtful attention is given to what it represents. For most purposes the interesting notion of validity is the one which coincides with the notion of logical truth. For modal systems where the modal operator is supposed to

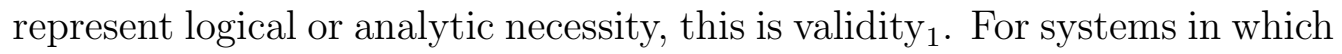
the modal operator represents some sort of "logically contingent" necessity (e.g., some type of nomic necessity) a notion resembling validity 2 coincides with the notion of logical truth. [1985: 27]

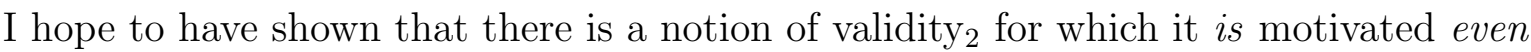
when $\square$ is to mean logical necessity. This is the notion of being true in all possibilities under all interpretations of the non-logical terms. As we have pointed out above, the essential difference between H\&H's approach and a standard Kripke Semantics, when it comes to the logically valid arguments and validities, is not really the insistence on using only $\mathcal{S}$, but rather, it lies in the truth clause for claims of the form $\square \alpha$. We have argued that the truth clause which $\mathrm{H} \& \mathrm{H}$ provide suffers from the same limitations as Cocchiarella and Hintikka's alternative semantics. If we accept that we are out to capture a phenomenon of being (necessarily) truth preserving under all interpretations of the non-logical terms, then this truth clause is illegitimate. ${ }^{164}$

\footnotetext{
164 A way to further strengthen the claim that the essential difference between validity and validity $_{2}$, when $\square$ is understood as logical necessity, is the truth clause given for $\square \alpha$ and not the use of $\mathcal{S}$, is to note that there seems to be no reason we could not define a modal operator which behaves as $\mathrm{H} \& H$ 's operator for logical necessity, while using various frames instead of $\mathcal{S}$. Let $C$ be the class of models over which we define validity 2 (and the corresponding notion of logical consequence). Let $\square_{H}$
} 
$\mathrm{H} \& \mathrm{H}$, as mentioned, argue that it is hard to discover any rationale behind the study of validity 2 , and these considerations are presented as being independent of the meaning of the modal operators in question. But as pointed out above, it is unclear that these considerations are independent of the supposed meaning of the modal operators, since they allow for continuing interest in "a notion resembling validity ${ }_{2}$ " when nomic necessity is at stake $([1985: 27])$. H\&H mention three overall ways in which we could motivate the study and use of universal validity, and argue that they all leave us wanting.

First off, $\mathrm{H} \& \mathrm{H}$ consider thinking of frames different than $\mathcal{S}$ as representing other ways that the space of possible worlds might have been. They deny this on grounds that "the whole point of invoking the possible worlds in the first place was to provide a foundation for our notion of possibility" [1985: 15]. However, completely independent of these issues, we can see that the motivation fails because it simply would not be correct to look at the different frames (on which we build our models) in a Kripke Semantics this way. The indices represent possible worlds in order to ensure that together the Kripke Models represent all and only combinations of possible circumstances and interpretations for the language. They do not represent different ways the class of possible worlds could have been. In order to secure that all such interpretations and circumstances are represented by a model, it is certainly possible to insist that each Kripke Model has a certain underlying frame, but it is not necessary. We shall look at be defined by the following truth clause: For $M \in C$ and $w \in W_{M} ; M, w \Vdash_{g} \square_{H} \alpha$ if and only if for all $M^{\prime} \in C, w^{\prime} \in W_{M^{\prime}}$ and variable assignments $g^{\prime} ; M^{\prime}, w^{\prime} \Vdash_{g^{\prime}} \alpha$. Of course, we would have to pay attention to the technical details in order to make sure that this remains well defined, but it should be clear that from the perspective of the resulting logic, $\square_{H}$ will behave like H\&H's operator for logical necessity: $\square_{H} \alpha$ will be true at a world if and only if $\alpha$ is true on all domains under all interpretations of the non-logical terms.

There therefore seems to be no reason why we could not generate the same semantics (same in terms of the class of valid arguments and validities) using various frames. But this, of course, is a purely technical point. $\mathrm{H} \& \mathrm{H}$ claim further that there seem to be no motivation behind the use of various frames and the resulting notion of validity ${ }_{2}$. We will look at this briefly below, but we may note that there seems to be a slight tension present in H\&H's work here, since, as we saw above, they seem also to claim that the output of a given semantics can justify its use. 
this further below.

As another motivation for validity $2, \mathrm{H} \& \mathrm{H}$ consider theories of how context can affect what is being expressed by a certain utterance or inscription of a sentence. Inspired by Stalnaker's work on possible worlds (Stalnaker [1976]), they suggest that the different frames are to be "thought of as representing the worlds possible relative to some context" ([1985: 17]). The idea is that context determines different sets of possible worlds as those that are relevant to the utterances and inscriptions.

$\mathrm{H} \& \mathrm{H}$ accept this way of motivating the use of different frames, but find it lacking in that "[w]e seriously doubt, however, that much of the formal work done on validity 2 in the last twenty-five years has been motivated by this way of understanding things" ([1985: 18]). There are of course two separate questions here: whether this kind of outlook has been the motivation behind studying validity 2 and whether it could be considered sufficient motivation. I suspect that $\mathrm{H} \& \mathrm{H}$ are correct in the first kind of claim, but this, of course, has no bearing on whether this could be a motivation behind validity $_{2}$. I will not pursue those issues here.

The third way of motivating the use of validity ${ }_{2}$ relies on seeing the difference between each frame as differences in the interpretation of the language. $\mathrm{H} \& \mathrm{H}$ call this the structural approach and claims:

On the structural approach, the mere fact that two model structures share certain formal features (e.g., that a certain relation between possible worlds and another relation between moments of time are both reflexive and transitive) is seen as sufficient for using both in determining whether a sentence is $\operatorname{valid}_{2}$. A valid 2 sentence is thus one which expresses a truth of logic when its intensional connectives are read in a variety of different ways. [1985: 15]

The idea is that a valid 2 sentence records more than one meaning of the intensional 
connectives and is true under all such understandings of these connectives. The different meanings of the connectives are given by the different frames used in the definition of validity.

Thus on the structural approach, validity ${ }_{2}$ can be thought of as giving a complete account only of what is common to the logic of a great many intensional connectives, most of which are never even specified. [1985: 15]

There are a couple of things to note about this. First off, H\&H speaks as if the different meanings of $\square$ which we are surveying are completely unrelated. This, of course, should not be accepted. In a Kripke Semantics $\square \alpha$ is true at $w$ if and only if $\alpha$ is true in all worlds that are $R$-related, and since this is the same in all models, we can hardly claim that the meanings of the $\square$-operators from model to model are completely unrelated. But one might accept the gist of these comments and deny that we can talk of the same meaning of two $\square$-operators in two different models at all. What Kripke Semantics then tells us is that no matter how the exact details concerning the modal space of interest shake out, if the semantics of the $\square$-operator is that of ranging over all the worlds that are possible from a given perspective, we can be sure that the valid argument preserve truth in virtue of this very meaning. One might even consider this a motivation for Kripke Semantics as opposed to H\&H's notion of validity ${ }_{1}$, since there is no consensus about the nature of the possible worlds which they employ in their semantics. From this perspective, then, the claim of the Kripke Semanticist would be that at least one frame captures the modal space in question, though perhaps only through idealization of the same kind present in the circumscription of $\mathcal{S}$. McKeon [2005] refers to this kind of defense of a Kripke Semantics as "a pluralistic defense," since we are now concerned with plural meanings of $\square$. H\&H argues that such a semantics would be of little interest, since presumably we are interested in just one meaning of $\square([1985: 15])$. Now, even if we accept that we should not speak about sameness of meaning between $\square$-operators in 
different Kripke Models, it does not follow that they are therefore completely unrelated. There is arguably some idealization involved in circumscribing the set of possible worlds on which we build our models, idealization which $\mathrm{H} \& \mathrm{H}$ explicitly endorse in their definition of $\mathcal{S}$, and this idealization will consequently be inherited by the meaning of $\square$. What $\mathrm{H} \& \mathrm{H}$ can claim for their semantics is that the idealized meaning of $\square$ is the same in all models. But while it is perhaps inappropriate to speak of the meaning of in a Kripke Semantics as having the same idealized meaning in all models, it does not follow that these idealized meanings are not idealizations of the same meaning. The meanings of $\square$ in different Kripke Models clearly are idealizations of the same meaning, a meaning which is given by the truth-clause for $\square$ : that of being true in all possible worlds relative to a given one.

If there is idealization involved in the meaning of $\square$ in a given model, then it is unclear that a model where $W$ is as specified by $\mathrm{H} \& \mathrm{H}$ does a better job at capturing this meaning than does a model where $|W|=1$. A model of the latter kind is not to be thought of as representing a way the world could have been where there is only one possible world. It represents a certain interpretation of the language under which all atomic formulae or their negations are necessary. If we want, we might even see this model as an idealization of a model containing multiple duplicates of the single index, where a duplicate is an index sharing the domain and the valuation of the language given there. In any case, there is certainly some idealization at work in a Kripke Semantics, but it is unclear that we gain anything from working with just one frame and the idealized meaning of $\square$ given on this.

Now, this might naturally invite questions as to what kind of entities the members of $\psi(@)$ can be (or represent) in a model where $|W|=1$, since they now exist (or are represented to exist) in all possible worlds. But this is not a question that is exclusive to Kripke Semantics, since the same kind of questions arise when using only $\mathcal{S}$ : what 
kind of entities, if any, are the ones that are allowed to be (or be represented to be) in the domains of all possible worlds?

Perhaps it would require a separate thesis to assess the claim of whether we should use just one frame in defining logical consequence and logical truth, or whether we should allow for different frames, such as it is standardly done in Kripke Semantics. We might want to note, in this relation, that the arguments declared valid on the two different approaches will differ minimally, when the truth of $\square \alpha$ is decided relative to just one model.

What I hope to have suggested is that it is unclear that $\mathrm{H} \& \mathrm{H}$ are correct in their claim that it is hard to discover any rationale for the use of universal validity, since they are misrepresenting the ways in which one could motivate such a use. The claim here is not that a Kripke Semantics is better motivated than H\&H's alternative semantics. Rather, the claim is that it is unclear that $\mathrm{H} \& \mathrm{H}$ establish that there seems to be no motivation behind a standard Kripke Semantics. In fact, I have no absolute reasons to offer against the use of just one frame as long as it is capable of capturing all interpretations of the language. In order to do so it is essential that all valuations on this frame are allowed and that the truth of $\square \alpha$ is decided relative to just one valuation on this frame. ${ }^{165}$ But this is just to say that if we are out to capture a notion of necessary truth preservation under all interpretations of the language, the approach taken in a standard Kripke Semantics - of letting the truth of $\square \alpha$ be decided relative to just one model that may not contain all possible first-order structures for the non-modal part of the language - is the correct one. As we pointed out, $\mathrm{H} \& \mathrm{H}$ do

\footnotetext{
${ }^{165}$ Such a semantics will validate the sentence $\diamond \exists x_{1} \ldots \exists x_{n}\left(x_{1} \neq x_{2} \wedge \ldots \wedge x_{1} \neq x_{n} \wedge \ldots \wedge x_{n-1} \neq x_{n}\right)$, for each $n \leq \tau$, as long as it is required that for each $n \leq \tau$, there is $w \in W$ s.t. $|\psi(w)|=\tau$. This might be considered motivation for using just one frame, but, again, it is not required to use just one frame to make these sentences valid. Instead, each frame on which we build our models would have to meet some minimal requirements.
} 
not develop their semantics of $\square$, understood as logical necessity, in accordance with this.

\subsubsection{Logical Possibility is Non-Relative}

McKeon [2005] develops a defense of universal validity qua logical truth in a standard Kripke Semantics for quantified languages.

The criticism, largely ignored in the literature, claims that when the box and diamond operators are interpreted as the logical modality operators, the Kripkean account is extensionally incorrect because it fails to reflect the fact that all sentences stating truths about what is logically possible are themselves logically necessary. [2005: 305]

The criticism which McKeon is referring to is certainly related to the criticism which we looked at above. McKeon cites Cocchiarella [1975], Hintikka [1982], and H\&H [1985] as critics, but McKeon's presentation of the criticism is made in terms of certain properties of logical necessity and possibility, rather than as a claim about the proper models over which to define consequence. In particular, McKeon focuses on the nonrelativeness of logical possibility. If it is true that $X$ is logically possible, then from all logically possible ways the world could have been, this is the case. Hence, in all logically possible ways the world could have been $X$ is logically possible, so it is logically necessary that $X$ is logically possible. This non-relativeness of logical possibility, as we saw above, is reflected in the 5-axiom: $\diamond A \rightarrow \square \diamond A$. In this section we will look at McKeon's presentation of the problem, his solution, and make some comments to sort out some potentially confusing claims made by McKeon.

McKeon asks us to consider the formula $\diamond \exists x(F x \wedge P x)$ interpreted as 'It is logically possible that someone is both female and U.S. President'. More precisely, in fact, McK- 
eon asks us to consider the interpreted sentence: $\diamond \exists x($ Female $(x) \wedge U . S . P r e s i d e n t(x))$ ([2005: 307]) (henceforth $\diamond \exists x(F e m(x) \wedge U S P(x)))$. This, obviously, is true. However, as McKeon points out, there are obviously Kripke Models, $M$, such that $M \Downarrow \diamond \exists x(F x \wedge$ $P x)$. Such a model would be given by the conditions of having $I(w)(F) \cap I(w)(P)=\emptyset$, for all $w \in W$, something which is perfectly acceptable from the perspective of Kripke Semantics. ${ }^{166}$ This means that $\diamond \exists x(\operatorname{Fem}(x) \wedge U S P(x))$ is true, but not a logical truth of the system. But, since logical possibility is non-relative, it should be. Again, whenever $A$ is logically possible, it is logically necessary that $A$ is logically possible, witnessed by the 5-axiom.

From our present perspective it should be easy to see what goes wrong in the above line of thought. Sure, $\exists x(F x \wedge P x)$ is logically possible under a certain interpretation, but this does not mean that its logical possibility should be a validity of the system (i.e., should be logically necessary). For this to be the case, all interpretations of $\diamond \exists x(F x \wedge P x)$ should be such that it is true in all possibilities, but there are surely interpretations for which this is not the case. Consider, for example, the interpretation of $F$ as 'is female', and $P$ as 'is non-female'. This interpretation is suggested by McKeon himself ([2005:313]). Under this interpretation $\diamond \exists x(F x \wedge P x)$ is not true. Another example which McKeon mentions is $\diamond \exists x \exists y(x \neq y)$. This formula is interpreted as stating that it is logically possible that there exists two or more objects. Since this is true, it should be logically necessary.

According to McKeon the criticism turns on what is reflected in the 5-axiom:

Logical facts can't be otherwise; they are not contingent. According to the criticism, an adequate logic of logical modality must reflect the following thesis about logical possibility (TLP):

\footnotetext{
${ }^{166}$ We may note, incidentally, that it is not acceptable from the perspective of the account that requires that all first-order models - whether they share a domain or not - are in the range of the quantifier determining the truth of $\square$-claims.
} 
All true statements about what is logically possible in first-order logic are logical truths.

The formal representation of (TLP) is the S5 characteristic axiom $\left\ulcorner\diamond_{S 5} \alpha \rightarrow\right.$ $\left.\square_{S 5} \diamond_{S 5} \alpha\right\urcorner$. Hence, the choice of S5 as the correct logic of logical modality is essential to the criticism of the Kripke account. [2005: 308]

We here see McKeon claiming that the choice of S5 as the correct logic of a logical necessity operator is essential to the criticism. At this point we might already suspect that there is some confusion going on here, since the $\square$-operator in a standard Kripke Semantics with $R$ a universal relation is an S5-modality.

[T] he criticism may be put as follows. An adequate account of logical truth for language $L$ must make it the case that for arbitrary non-modal $L$-sentences $\alpha$, if $\left\ulcorner\diamond_{S 5} \alpha\right\urcorner$ is true, then $\left.\triangleright^{\ulcorner} \diamond_{S 5} \alpha\right\urcorner$ is logically true. (TLP) fails on the Kripkean account because on the Kripkean account, $\left.{ }^{\ulcorner} \diamond_{S 5} \alpha\right\urcorner$ is a logical truth (because it is universally valid), only if $\alpha$ is a first-order logical truth.

$[S]$ ince the Kripke account does not reflect (TLP), it is reasonable to think that it is inaccurate. [2005: 308-309]

Again, it is unclear what the latter part of this quote could mean. If reflecting (TLP) just means having the 5-axiom as a validity - as McKeon himself seems to suggest in the previous quote - a standard Kripke Semantics for a logical necessity operator does reflect (TLP).

Before we turn to sort these issues out, let us make a few brief comments on McKeon's solution. McKeon suggests that just as the domain of quantification is varied when we decide extensional first-order logical truths, the domain of quantification (over 
worlds) should equally be varied in a modal setting. The analogy is this: when deciding the logical truth of $\forall x \alpha$ in an extensional language, we survey all possible domains that the initial quantifier could range over. Likewise, when we decide the logical truth of $\square \alpha$ in a modal language we should survey all possible collections of worlds that $\square$ could range over. This leads McKeon to accept the principle (LFP') ([2005: 313]).

(LFP') If $\varphi$ is a modal sentence and $\psi$ is obtained from it either by the substitution of formulas for non-logical predicates, or by uniform non-empty restrictions of the ranges of all modal quantifiers then if $\varphi$ is logically true, so is $\psi$.

The first part of this principle states that logical truth is closed under uniform substitution. The second part requires that a logical truth remains valid upon any restriction of the range of the modal operators. The first part rules out $\diamond \exists x(\operatorname{Fem}(x) \wedge U S P(x))$ as a logical truth. If we substitute 'non-female' for 'U.S. President' the result is false. ([2005: 313]). The latter part secures that $\diamond \exists x \exists y(x \neq y)$ is not a logical truth, since the domain over which $\diamond$ ranges could contain just one world with just one individual. ([2005: 313]). Now, (LFP'), according to McKeon, is in tension with (TLP), which McKeon in turn identifies with having the 5-axiom. The idea, it seems, is that (TLP) says that $\diamond \exists x(\operatorname{Fem}(x) \wedge U S P(x))$ and $\diamond \exists x \exists y(x \neq y)$ are logical truths, whereas according to (LFP'), they are not.

On my view, the correct logic of logical modality is at least as strong as S4, but not as strong as S5. [2005: 313]

Now, it is unclear that (LFP') is in tension with (TLP), at least insofar as we identify satisfying the latter with having the 5 -axiom valid, and consequently, it is unclear why McKeon wants to give up on S5 as the correct semantics of logical necessity. The problem seems to be that the content of (TLP), as McKeon intends to understand it, is not given by the 5-axiom. 
There is some confusion here between the object language meaning of $\square$ and the meta-language ascription of validity (what McKeon calls 'logical truth') to a formula. Let us use logical truth as a property of natural language sentences or formulae under a given interpretation, and validity as a property of formulae of the formal language. Logical truth, as we use it here, means that a statement is true in virtue of meaning; it could not be false, or equivalently, it is logically necessary. If (LFP') is understood to be about validity, then it is not in tension with the 5-axiom. It states that validity is closed under substitution and that a validity remains valid when we restrict the range of the quantifier in the truth clause for $\square$ claims. We may note that both of these claims are true for a standard Kripke Semantics which also has the 5-axiom valid. Such a semantics is closed under substitution and the restriction of the quantifier in the truth clause for $\square$ is guaranteed by the very definition of universal validity as truth in all models.

However, this does not seem to be the way that McKeon intends to understand (LFP'), since he uses it to explain why the interpreted sentence $\diamond \exists x(F e m(x) \wedge U S P(x))$ is not a logical truth. Hence, it seems that the sentences mentioned in (LFP') are meant to be formal sentences under a given natural language interpretation. But if (LFP') is understood to be about logical truth understood as logical necessity, then it does not seem that (LFP') is straightforwardly true. While 'It is logically possible that someone be both female and U.S. President' is surely a logical truth in the sense of being true in virtue of meaning, the corresponding sentence 'It is logically possible that someone be both female and non-female' is not. Yet, the latter is just the former with 'non-female' substituted for 'U.S. President'. It is clear that from the perspective developed in this thesis we cannot accept (LFP') understood in this way. 
Now, the reason McKeon thinks that the crux of the criticism is the 5-axiom, seems to rest on an insistence on equating the meaning of the meta-language ascription of validity to a formula with the object language occurrence of $\square$, and as we have argued extensively, no such conflation is forced upon us in a standard Kripke Semantics. From such a perspective the validity of the 5 -axiom means that there are no interpretations of the non-logical parts such that it expresses a falsehood. It does not state, that whenever a formula $\diamond \alpha$ is true, then it is also a logical truth. How could it? $\diamond \alpha$ is a formula. It is not the kind of thing that can be true or false. It can be true or false under an interpretation, and as we have seen, if it is true under an interpretation, then that same interpretation will say that it is logically necessary that it is true.

McKeon's examples, though, seem to run the two together. If $\diamond \exists x(F x \wedge P x)$ is true under an interpretation corresponding to the reading $R_{I}(\diamond \exists x(F x \wedge P x))=$ 'It is logically possible that someone is both female and U.S. President', then what follows is not, as McKeon suggests, that $\diamond \exists x(F x \wedge P x)$ should be a validity, but that $\square \diamond \exists x(F x \wedge P x)$ should be true under the same interpretation as makes the first true, an interpretation which, through the meaning of $\square$, corresponds to $R_{I}(\square \diamond \exists x(F x \wedge P x))=$ 'It is logically necessary that it is logically possible that someone is both female and U.S. President'. But a standard Kripke Semantics will validate this. Any model making the first true, and therefore any model which interprets $\diamond \exists x(F x \wedge P x)$ in accordance with $R_{I}$, will also make the latter true.

On the other hand, since Kripke Semantics validates the 5-axiom, it does follow that if $\diamond \alpha$ is a validity, then $\square \diamond \alpha$ will be a validity too. But this result should be endorsed. If $\diamond \alpha$ is true in all possibilities under all interpretations, then given the non-relativity of logical possibility, $\square \diamond \alpha$ will be true in all possibilities under all interpretations.

What McKeon really means to be conferring by the content of (TLP) seems to be the following point: if a non-modal $\alpha$ is satisfiable in first-order logic, then $\diamond \alpha$ should 
be a validity. And while it is true that in a standard Kripke Semantics for logical necessity this will not hold, we can see how this is just a restatement of the conditions put on the modal operators in the semantics of Cocchiarella, Hintikka, and H\&H, respectively: the modal operators should range over all first-order structures. ${ }^{167}$ If the content of (TLP) is given in this manner, then it would be incorrect to conflate it with the validity of the 5-axiom, as we have explained above. The 5-axiom does not state that if a non-modal $\alpha$ is satisfiable in first-order logic, then $\diamond \alpha$ should be a validity. There is no reason to deny the axiom $\diamond A \rightarrow \square \diamond A$ from the perspective of Kripke Semantics, and neither is there any reason to give up on the non-relativity of logical possibility. These two arguably go hand in hand, but not in the manner suggested by McKeon.

We may want to note, though, that McKeon's solution, although we have argued that it is mistaken, was able to explain why $\diamond \exists x \exists y(x \neq y)$ should not be a logical truth. From our present perspective, we cannot straightforwardly explain why $\diamond \exists x \exists y(x \neq y)$ is not valid. The sentence consists of only logical constants which all receive the same interpretation in all models. The interpretation enjoyed by this sentence is such that it is consistent with the reading 'It is logically possible that there are at least two things'. Now, if we agree that this is true, the formal sentence is therefore true in all possibilities under all interpretations, and it seems it should be a validity, though in a standard Kripke Semantics it is not. Any model such that for all $w,|\psi(w)|=1$ will make it false. What happens here is that the logical terminology is expressive enough to be sensitive to the mode of representation of a given model, that is, the logical

\footnotetext{
${ }^{167}$ Properly speaking, Cocchiarella's semantics (Cocchiarella [1975]) does not have the requirement that the modal operators range over all first-order structures, only all first-order structures sharing a domain. For this reason, Cocchiarella's semantics will not satisfy the principle that if a non-modal $\alpha$ is satisfiable in first-order logic, then $\diamond \alpha$ should be a validity. Consider $\exists x \exists y(x \neq y)$. This is satisfiable, but in Cocchiarella's semantics $\diamond \exists x \exists y(x \neq y)$ will not be true in a model, $\langle D, I\rangle$, where $|D|=1$, and hence it will not be true in all models.
} 
terminology can, so to speak, express facts about $\psi(w)$ for $w \in W$. If we accept that 'It is logically possible that there are at least two things' is a logical truth, and so consequently that $\diamond \exists x \exists y(x \neq y)$ should be valid, then we ought to make the requisite changes to the semantics. To exclude the interpretations of the latter under which it is false, and to secure similar interpretations of other cardinality sentences, we will have to require that for each non-zero countable cardinal, $\tau$, there is $w \in W$ s.t. $|\psi(w)|=\tau$.

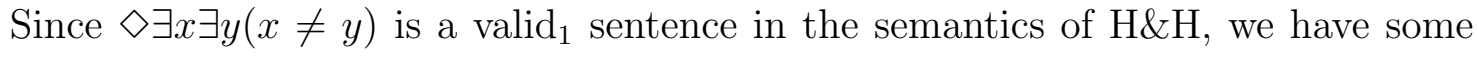
vindication of the approach they take to modal semantics. But, of course, the example does not show that we ought to construct all models on the same frame, $\mathcal{S}$, but only that since our language is now expressive enough to make claims about the way in which a single model represents, some models ought to be excluded as they are not proper idealizations. It is a claim that there should be further minimal restrictions put on the models, not a claim that there should be only one frame involved. Say that a sentence is aug-valid just when it is true in all Kripke Models meeting the constraints that for any non-zero countable cardinal, $\tau$, there is $w \in W$ s.t. $|\psi(w)|=\tau$. Clearly all universally valid sentences will be aug-valid, since the models over which we define the former include the models over which we define the latter. As long as we allow the valuations of the non-logical terminology at the indices to vary arbitrarily, there will be minimal changes to the output of the semantics. I dare conjecture that the only "new" validities (i.e., aug-validities that are not universally valid) will be sentences expressing facts about the various cardinalities of the domains of the indices, such as $\diamond \exists x_{1} \ldots \exists x_{n}\left(x_{1} \neq x_{2} \wedge \ldots \wedge x_{1} \neq x_{n} \wedge \ldots \wedge x_{n-1} \neq x_{n}\right)$, for each $n \leq \tau$, and, obviously, any tautologies that can be defined using these sentences once it is accepted that they are tautologies: such as $\square \alpha \vee \diamond \exists x \exists y(x \neq y),(\neg \alpha \vee \alpha) \wedge \diamond \exists x \exists y \exists z(x \neq y \wedge x \neq z \wedge y \neq z)$, and the like. 


\section{CONCLUSION}

In the present thesis we have been concerned with model-theoretic characterizations of logical consequence. Our primary aim has been to defend the use of a standard Kripke Semantics in characterizing the logical consequence relation for a language augmented with a $\square$-operator to be understood as logical necessity. This defense was grounded in Etchemendy's distinction between interpretational and representational model-theoretic semantics.

In chapter one we set out and defended Etchemendy's criticism of purely interpretational model-theoretic characterizations of logical consequence. By purely interpretational we mean model-theoretic characterizations that view the changes between models only as changes in interpretation of the non-logical terminology, all from the perspective of the actual world. We argued, following Etchemendy, that such characterizations miss the most crucial aspect of logical consequence, namely, that of guaranteeing the truth of the conclusion upon the truth of the premises, both in actual and counterfactual circumstances. We concluded that if logically valid arguments are to possess the characteristics that we normally associate with them-most prominently that of telling us how the world would have to be if certain other (perhaps non-actual) things were the case - then there must be a representational aspect to the underlying model theory. The models must represent other states of affairs.

In the second chapter we set out a positive view of a model theory that has a representational aspect to it. We based this primarily on Etchemendy's positive views 
of model theory, but we also saw that the view is endorsed by other theorists. By allowing for some terms to be treated as non-logical we reintroduce an interpretational aspect to the model theory, and the notion of a logically valid argument that our model theory captures is consequently that of being truth preserving in all possibilities under all interpretations of the non-logical terminology. However, as we also saw, the dividing line between being a logical term and being a non-logical term is, at least for Etchemendy, a pragmatic matter, as the important notion is still truth preservation in virtue of the meaning of all terms. But if an argument is truth preserving in virtue of the meaning of some terms, then it will be truth preserving in virtue of all terms, it is just that the exact meaning of those additional terms does not matter.

Using this informal notion as that which our model theory aims to capture we provided arguments to the effect that our model theory does not declare any genuinely invalid arguments valid. That is, we do not have overgeneration. As for the opposite question of undergeneration, that is, of whether our model theory declares some genuinely valid arguments invalid, we found that this should be expected. If a genuinely valid argument preserves truth in virtue of the meaning of all terms, and our model theory captures the phenomenon of being truth preserving in virtue of the terms currently treated as logical constants only, then we should expect that there could be arguments that are in fact valid, but will not be declared so. This happens when the truth preservation of an argument is due to the meaning of a term the counterpart of which is treated as a non-logical constant. But this latter fact, that there is undergeneration, also let us deny that the theorems stating that there is no undergeneration and no overgeneration are true by default. The former is false, the latter true.

In the third, brief, chapter we looked at the issue of the underlying models from a perspective where they are meant to both represent and interpret. We argued that there are straightforward ways to see a model as delivering an interpretation for the 
language, and equally straightforward ways to see a model as representing a possible way the world could have been. However, in the case in which a model is meant to both represent and interpret, we found that we could not straightforwardly combine these two approaches - at least under the natural assumption that models, whatever else they may be, are constructed out of actually existing objects. Instead we presented a theory of how models both represent and interpret where the representational and interpretational capacities come about through certain modal facts: facts about how the model would relate to reality had reality been different in certain respects. We pointed out that this approach is perfectly acceptable from the modeling perspective of Etchemendy.

In the fourth chapter we applied these lessons to a model theory aimed at capturing consequence and validity in a modal language augmented with a $\square$-operator to mean logical necessity. We argued that there is an interesting notion which Kripke Semantics can be seen as capturing: that of being truth preserving in all possibilities under all interpretations of the language. We made this point by comparing it to a claim that all possible first-order structures should be in the range of the quantifier in the truth clause for $\square$-claims, and we found that this would be an illegitimate demand from our current perspective, as it would exclude certain interpretations of the language from being represented by a model. The variation in possible structures over which the quantifier in the truth clause for $\square$-claims range is needed to secure that all possible interpretations of the non-logical constants are represented by some model.

In the last part of the chapter we argued that if there is a debate to be had concerning the adequacy of Kripke Semantics for modeling our notion of logical consequence, it concerns whether all models are proper idealizations of a possible way the world could have been relative to an interpretation of the language. And while we found that there are certain formal sentences, such as $\exists x \exists y(x \neq y)$, which suggest the need for putting 
further minimal constraints on our models - if we want to capture a notion of $\square$ as logical necessity - we argued that this does not in itself warrant the claim that there should be only one frame involved in deciding validity and logical consequence. And even if there should, we pointed out that the approach taken in Kripke Semantics, of letting valuations of the language vary arbitrarily throughout the indices, is the correct approach. 


\section{REFERENCES}

Barcan, R. 1946. 'A Functional Calculus of First Order Based on Strict Implication', in Journal of Symbolic Logic, vol 11.

Barwise, J. \& Etchemendy, J. 1989. 'Model-Theoretic Semantics', in Posner, M. (ed.): Foundations of Cognitive Science. MIT Press.

Beall, J. \& Restall, G. 2006. Logical Pluralism. Oxford University Press.

Blanchette, P. 2000. 'Models and Modality', in Synthese, vol 124, issue 1-2.

Burgess, J. 1999. 'Which Modal Logic is the Right One?', in Notre Dame Journal of Formal Logic, vol 40, no 1.

Burgess, J. 2003. 'Which Modal Models are the Right Ones (for Logical Necessity)?', in Theoria, vol 18, no 2.

Carnap, R. 1946. 'Modalities and Quantification', in Journal of Symbolic Logic, vol 11 .

Carnap, R. 1947. Meaning and Necessity. Chicago: University of Chicago Press. 2nd edition with supplements 1956.

Chihara, C. 1998. The Worlds of Possibility: Modal Realism and the Semantics of Modal Logic. Oxford University Press. 
Cocchiarella, N. 1975. 'On the Primary and Secondary Semantics of Logical Necessity', in Journal of Philosophical Logic, vol 4.

Cocchieralla, N. 2002. 'Logical Necessity Based on Carnap's Criterion of Adequacy', in Korean Journal of Logic, vol 5, no 2.

Creath, R (ed.). 1990. Dear Carnap, Dear Van - The Quine-Carnap Correspondence and Related Work. University of California Press.

Cresswell, M. 1972. 'The World is Everything That is the Case' in Australasian Journal of Philosophy, vol 50, no 1.

Davidson, D. 1973. 'In Defense of Convention T', in Studies in Logic and the Foundations of Mathematics, vol 68.

Edwards, J. 2003. 'Reduction and Tarski's Definition of Logical Consequence', in Notre Dame Journal of Formal Logic, vol 44, no 1.

Etchemendy, J. 1982. Tarski, Model Theory, and Logical Truth, PhD dissertation, Stanford University.

Etchemendy, J. 1983. 'The Doctrine of Logic as Form', in Linguistics and Philosophy, vol 6.

Etchemendy, J. 1988a. 'Models, Semantics and Logical Truth', in Linguistics and Philosophy, vol 11.

Etchemendy, J. 1988b. 'Tarski on Truth and Logical Consequence', in The Journal of Symbolic Logic, vol 53, no 1.

Etchemendy, J. 1990. The Concept of Logical Consequence. CSLI Publications. Reprint Edition: 1999. 
Etchemendy, J. 2008. 'Reflections on Consequence', in Patterson, D. (ed.): New Essays on Tarski and Philosophy. Oxford University Press.

Glanzberg, M. forthcoming. In Hjortland, O. \& Caret, C. (eds.): Foundations of Logical Consequence. Oxford University Press.

Gómez-Torrente, M. 1996. 'Tarski on Logical Consequence', in Notre Dame Journal of Formal Logic, vol 37, no 1.

Gómez-Torrente, M. 1998. 'On a Fallacy Attributed to Tarski', in History and Philosophy of Logic, vol 19.

Gómez-Torrente, M. 2002. 'The Problem of Logical Constants', in The Bulletin of Symbolic Logic, vol 8.

Griffiths, O. 2014. 'Formal and Informal Consequence', in Thought, vol 3.

Hale, B. 1996. 'Absolute Necessities', in Nô̂s, vol 30 (Supplement: Philosophical Perspectives, 10, Metaphysics).

Hanson, W. 1997. 'The Concept of Logical Consequence', in The Philosophical Review, vol 106, no 3 .

Hanson, W. \& Hawthorne, J. 1985. 'Validity in Intensional Languages: A New Approach', in Notre Dame Journal of Formal Logic, vol 26, no 1.

Harman, G. 1984. 'Logic and Reasoning', in Synthese, vol 60.

Hintikka, J. 1961. 'Modalities and Quantification', in Theoria, vol 27.

Hintikka, J. 1980. 'Standard vs. Nonstandard Logic: Higher-Order, Modal, and First-Order Logics' in Agazzi, E. (ed.): Modern Logic - A Survey. D. Reidel Publishing Company. 
Hintikka, J. 1982. 'Is Alethic Modal Logic Possible?' in Acta Philosophica Fennica, vol 35.

Hodges, W. 1997. A Shorter Model Theory. Cambridge University Press.

Kanger, S. 1957. Provability in Logic. Acta Universitatis Stockholmiensis, Stockholm Studies in Philosophy, vol 1, Stockholm: Almqvist and Wiksell.

Kaplan, D. 1977. 'Demonstratives - An Essay on the Semantics, Logic, Metaphysics, and Epistemology of Demonstratives and Other Indexicals', in Almog, J. et al. (eds): Themes From Kaplan. 1989. Oxford University Press.

Kaplan, D. 1978. 'Transworld Heir Lines', in Loux, M. (ed.): The Possible and the Actual - Readings in the Metaphysics of Modality. 1979. Cornell University Press.

Kaplan, D. 1989. 'Afterthoughts', in Almog, J. et al. (eds): Themes From Kaplan. 1989. Oxford University Press.

Kneale, W. 1961. 'Universality and Necessity', in The British Journal for the Philosophy of Science, vol 12, no 46.

Kreisel, G. 1967. 'Informal Rigour and Completeness Proofs', in Lakatos, I. (ed.): Problems in the Philosophy of Mathematics. North-Holland Publishing.

Kripke, S. 1963. 'Semantical Considerations on Modal Logic', in Acta Philosophica Fennica, vol 16.

Kripke, S. 1980. Naming and Necessity. Harvard University Press.

Kripke, S. 2013. Reference and Existence. Oxford University Press. 
Lewis, C. 1918. A Survey of Symbolic Logic. Berkeley: University of California Press.

Lewis, C. \& Langford, C. 1932. Symbolic Logic. London: Century. 2nd edition 1959, New York: Dover.

Lewis, D. 1986. On the Plurality of Worlds. Blackwell Publishing.

MacFarlane, J. 2000. 'What is Modeled by Truth in All Models?', unpublished manuscript, http://johnmacfarlane.net/work.html.

Marcus, R. 1967. 'Essentialism in Modal Logic', in Nô̂s, vol 1, no 1.

McFetridge, I. 1990. 'Logical Necessity: Some Issues', in McFetridge, I. (Haldane, J. \& Scruton, R (eds.)): Logical Necessity and Other Essays. Aristotelian Society.

McGee, V. 1992. 'Two Problems with Tarski's Theory of Consequence', in Proceedings of the Aristotelian Society, New Series, vol 92.

McKeon, M. 2005. 'A Defense of the Kripkean Account of Logical Truth in First-Order Modal Logic', in Journal Of Philosophical Logic, vol 34, no 3.

McKeon, M. 2010. The Concept of Logical Consequence - An Introduction to Philosophical Logic. Peter Lang Publishing.

Mendelson, E. 1997. Introduction to Mathematical Logic. Fourth Edition. Chapman \& Hall/CRC.

Menzel, C. 1990. 'Actualism, Ontological Commitment, and Possible Worlds Semantics', in Synthese, vol. 85. 
Menzel, C. 2008. 'Actualism', in Zalta, E (ed.): Stanford Encyclopedia of Philosophy (Winter 2008 Edition). Stanford University 2008.

http://plato.stanford.edu/archives/win2008/entries/actualism

Plantinga, A. 1974. The Nature of Necessity. Oxford University Press.

Pollock, J. 1967. 'Logical Validity in Modal Logic', in The Monist, vol 51, no 1.

Priest, G. 1995. 'Etchemendy and Logical Consequence', in Canadian Journal of Philosophy, vol 25, no 2.

Prior, A. 1957. Time and Modality, Oxford: Clarendon Press.

Prior, A. 1964. 'Conjunction and Contonktion Revisited', in Analysis, vol 24, no 6 .

Quine, W. 1947. 'The Problem of Interpreting Modal Logic', in Journal of Symbolic Logic, vol 12.

Quine, W. 1960. Word and Object. Harvard University Press.

Quine, W. 1970. Philosophy of Logic. Prentice Hall.

Ray, G. 1996a. 'Logical Consequence: A Defense of Tarski', in Journal of Philosophical Logic, vol 25, issue 6.

Ray, G. 1996b. 'On the Possibility of a Privileged Class of Logical Terms', in Philosophical Studies, vol 81.

Ray, G. 1996c. 'Ontology-Free Modal Semantics', in Journal of Philosophical Logic, vol 25. 
Read, S. 1994. 'Formal and Material Consequence', in Journal of Philosophical Logic, vol 23.

Russell, G. 2008. 'One True Logic?', in Journal of Philosophical Logic, vol 37.

Sagi, G. 2011. 'Sher and Shapiro on Logical Terms', in Pelis, M. \& Puncochar, V. (eds.): The Logica yearbook 2010. College Publications.

Sagi, G. 2013. Logical Consequence: Between Formal and Natural Language, $\mathrm{PhD}$ dissertation, Hebrew University of Jerusalem.

Segerberg, K. 1971. An Essay in Classical Modal Logic, vols 1, 2, 3, Filosofiska Studier, 13, Uppsala: Uppsala Universitet.

Shalkowski, S. 2004. 'Logic and Absolute Necessity', in The Journal of Philosophy, vol 101, no 2.

Shapiro, S. 1998. 'Logical Consequence: Models and Modality', in Schirn, M. (ed.): The Philosophy of Mathematics Today. Clarendon Press.

Shapiro, S. 2001. 'Modeling and Normativity: How Much Revisionism Can We Tolerate?', in Agora : Papeles de Filosofia, vol 20, no 1.

Shapiro, S. 2002. 'Necessity, Meaning, and Rationality: The Notion of Logical Consequence' in Jacquette, D. (ed.): A Companion to Philosophical Logic. Blackwell Publishing Ltd.

Shapiro, S. 2005. 'Logical Consequence, Proof Theory, and Model Theory', in Shapiro, S. (ed.): The Oxford Handbook of Philosophy of Mathematics and Logic, Oxford University Press.

Shapiro, S. 2014. Varieties of Logic. Oxford University Press. 
Sher, G. 1991. The Bounds of Logic: A Generalized Viewpoint. MIT Press.

Sher, G. 1996. 'Did Tarski commit "Tarski's Fallacy"?', in The Journal of Symbolic Logic, vol 61, no 2.

Sher, G. 2003. 'A Characterization of Logical Constants Is Possible', in Theoria, vol 18, no 2 .

Sher, G. 2008. 'Tarski's Thesis', in Patterson, D. (ed.): New Essays on Tarski and Philosophy. Oxford University Press.

Smiley, T. 1998. 'Conceptions of Consequence', in Craig, E. (ed.): The Routledge Encyclopedia of Philosophy, vol 2. Routledge.

Smith, P. 2011. 'Squeezing Arguments', in Analysis, vol 71, no 1.

Stalnaker, R. 1976. 'Possible Worlds', in Nous, vol 10.

Tarski, A. 1956a. 'On The Concept of Logical Consequence', in Tarski, A.: Logic, Semantics, Metamathematics: Papers from 1923-1938. Translated by J. Woodger. Oxford University Press.

Tarski, A. 1956b. 'The Concept of Truth in Formalized Languages', in Tarski, A.: Logic, Semantics, Metamathematics: Papers from 1923-1938. Translated by J. Woodger. Oxford University Press.

Tarski, A. 1986. 'What are Logical Notions?' (edited by Corcoran, J.), in History and Philosophy of Logic, vol 7, issue 2.

Williamson, T. 2007. The Philosophy of Philosophy. Blackwell Publishing. 